\title{
Host Pathogen responses of chicken cells in vitro and in vivo to a diverse population of Campylobacter strains
}

\author{
John, Daniel A.
}

How to cite:

John, Daniel A. (2018) Host Pathogen responses of chicken cells in vitro and in vivo to a diverse population of Campylobacter strains. Doctoral thesis, Swansea University.

http://cronfa.swan.ac.uk/Record/cronfa50200

Use policy:

This item is brought to you by Swansea University. Any person downloading material is agreeing to abide by the terms of the repository licence: copies of full text items may be used or reproduced in any format or medium, without prior permission for personal research or study, educational or non-commercial purposes only. The copyright for any work remains with the original author unless otherwise specified. The full-text must not be sold in any format or medium without the formal permission of the copyright holder. Permission for multiple reproductions should be obtained from the original author.

Authors are personally responsible for adhering to copyright and publisher restrictions when uploading content to the repository.

Please link to the metadata record in the Swansea University repository, Cronfa (link given in the citation reference above.)

http://www.swansea.ac.uk/library/researchsupport/ris-support/ 


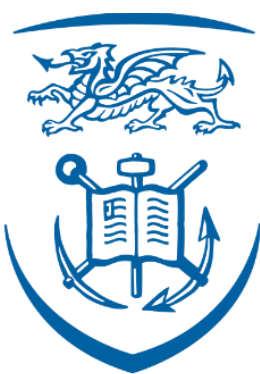

\section{Swansea University Prifysgol Abertawe}

Host pathogen responses of chicken cells in vitro and in vivo to a diverse population of Campylobacter strains

Daniel John

Submitted to Swansea University in fulfilment of the requirements for the Degree of Doctor of Philosophy

Swansea University 


\begin{abstract}
Campylobacter is the leading cause of bacterial food-borne diarrheal disease worldwide, with the bacteria being pathogens in humans and chickens. There are differences in the mechanistic understanding between Campylobacter strains, due, in part, to genomic and phenotypic strain diversity, which leads to inconsistent findings. This study aimed to understand underlying infection biology in host-pathogen interactions between populations of Campylobacter and the chicken host. A collection of Campylobacter jejuni strains were used to investigate the genotypic and phenotypic differences which yielded a diverse pan-geome of 2715 genes with genome wide association studies uncovering genes related to flagella assocaited with strains isolated from both humans and chickens. The $C$. jejuni strains were then used with a new commercially available chicken cell line to investigate the chicken systems with host responses measured in comparison to human systems. The pathogenic diversity of $C$. jejuni was measured through their inflammatory, cytotoxicity, adhesion, invasion and signalling responses in a high-throughput model using avian and human intestinal epithelial cells. C. jejuni induced IL-8 and CXCLi1/2 in human and avian epithelial cells, respectively, in a MAP kinase-dependent manner. In contrast, IL-10 responses in both cell types were PI 3kinase/Akt-dependent. C. jejuni strains showed diverse levels of invasion with high invasion dependent on MAP kinase signaling in both cell lines. C. jejuni induced diverse cytotoxic responses in both cell lines with cdt-positive isolates showing significantly higher toxicity. Blockade of endocytic pathways suggested that invasion by $C$. jejuni was clathrin- and dynamin-dependent but caveolae- independent in both cells. In contrast, IL-8 (and CXCLi1/2) production was dependent on clathrin, dynamin, and caveolae. Strong correlations were found between IL-8 with invasion and toxicity responses with GWAS being able to associated genes to expression of IL-8,-10 and level of invasion. Campylobacter strains were isolated from the caeca, ileum and liver and a pan-genome created with GWAS analysis discovering genes relating to iron transport and heat shock proteins in strains from the liver. Subsequently, caecal tonsils were extracted from chickens uninfected and chickens infected with a cocktail of six Campylobacter strains with a Campylobacter effect found where infected chickens produced significantly higher $T_{h} 1, T_{h} 2$ and $T_{h} 17$ cytokines. The work in this thesis contributes invaluable knowledge on the chicken innate immune system in response to Campylobacter and how similar host defence pathways to humans are utlised. The diversity found strongly indicates that a 'one strain fits all' approach to in vitro and in vivo experimental infections may not give accurate results on Campylobacter pathogenesis. Campylobacter isolates can use host immune responses to its advantage and to move from the gut of chickens to infect edible tissues such as the liver.
\end{abstract}




\section{Declaration}

This work has not previously been accepted in substance for any degree and is not being concurrently submitted in candidature for any degree.

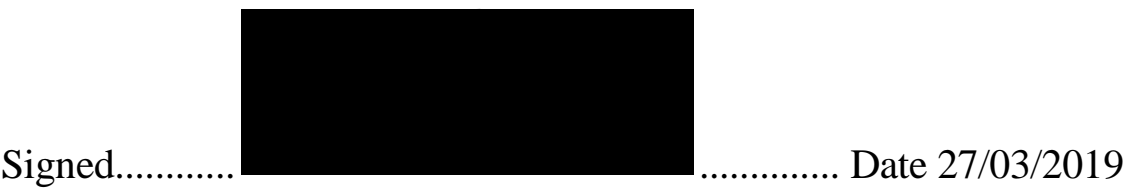

This thesis is the result of my own investigations, except where otherwise stated. Where correction services have been used, the extent and nature of the correction is clearly marked. Other sources are acknowledged by footnotes giving explicit references. A bibliography is appended.

Signed Date $27 / 03 / 2019$

I hereby give consent for my thesis, if accepted, to be available for photocopying and for inter-library loan, and for the title and summary to be made available to outside organisations following the expiry of the bar on access.

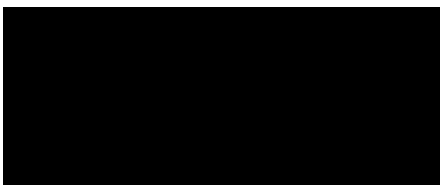

Date 27/03/2019. 


\section{Contents}

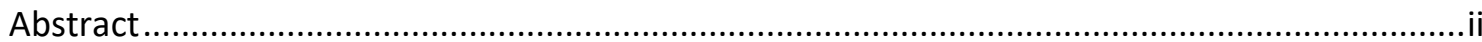

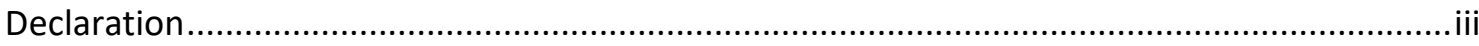

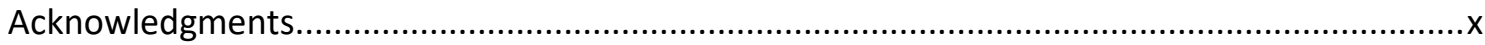

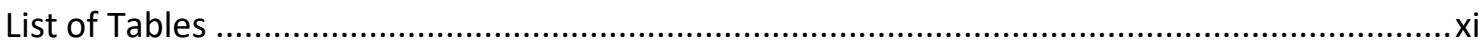

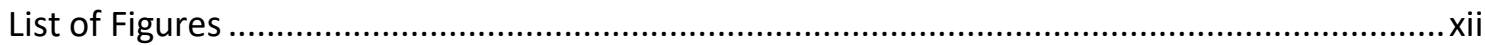

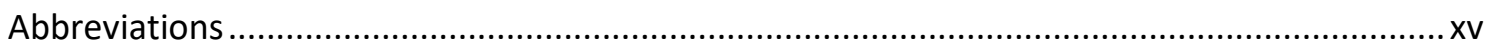

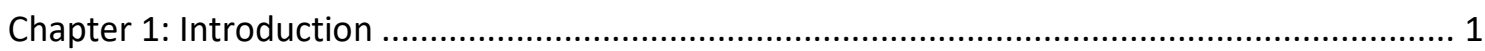

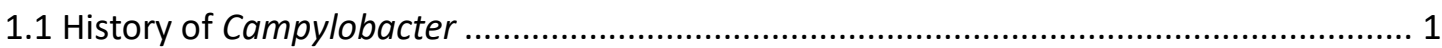

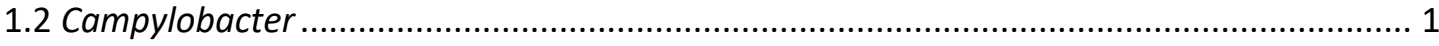

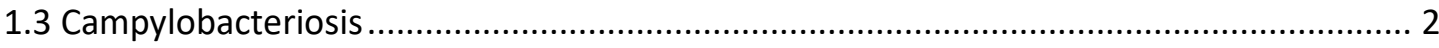

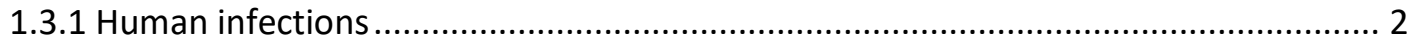

1.3.1 Chickens and Campylobacter ............................................................... 3

1.4 Campylobacter in different hosts ................................................................... 4

1.5 Campylobacter Genomics ................................................................................ 4

1.6 Isolation and identification of Campylobacter........................................................ 5

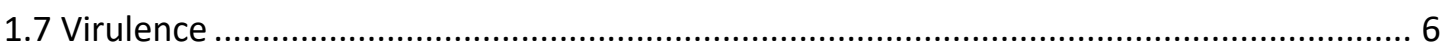

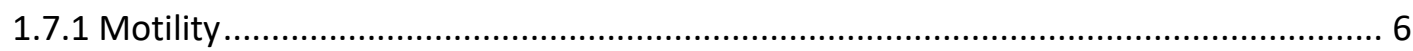

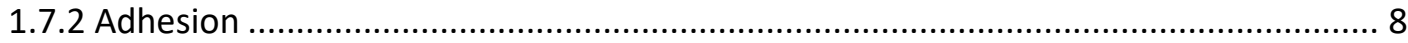

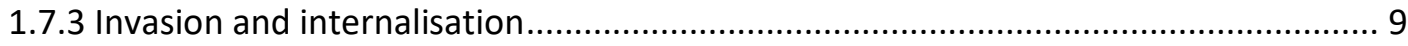

1.7.4 Intracellular survival......................................................................... 13

1.7.6 Endotoxin and exotoxin production .......................................................... 14

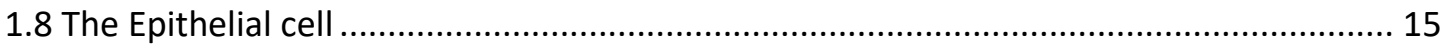

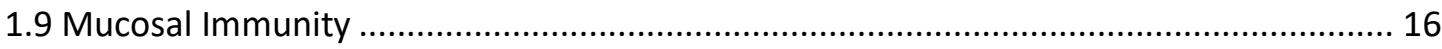

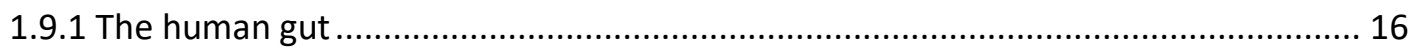

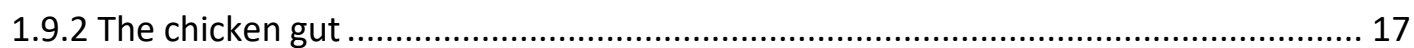

1.10 Toll-Like Receptors and signalling .............................................................. 20

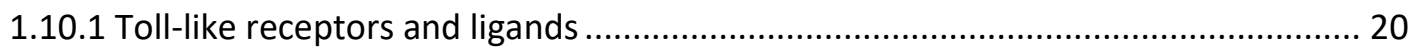

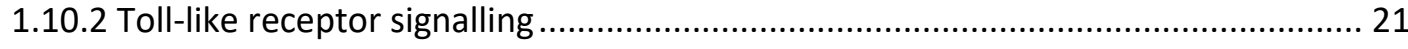

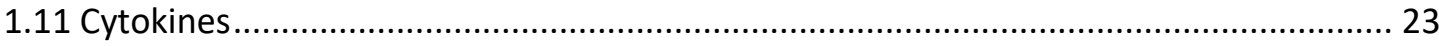

1.11.1 Structural groups based on receptors ..................................................... 23

1.11.2 Functional groups based on protective immunity ......................................... 23

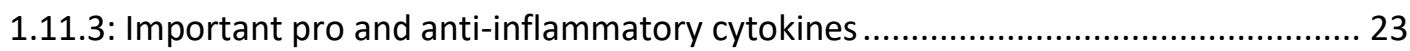


1.12 Aims and objectives

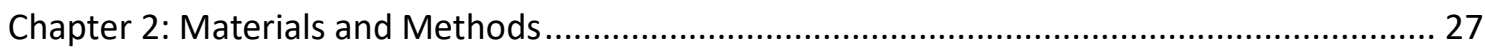

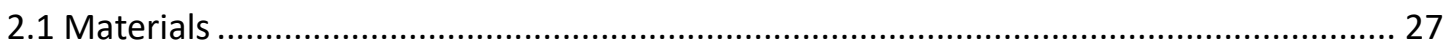

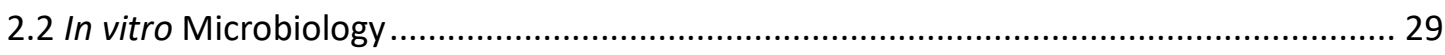

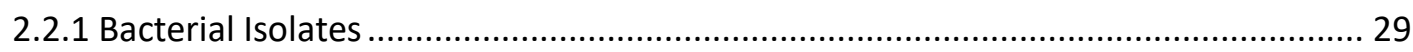

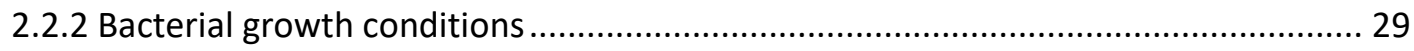

2.2.3 Culture of human and chicken intestinal epithelial cells ......................................... 34

2.2.4 Infection studies: co-culture of epithelial cells and Campylobacter.......................... 34

2.2.5 IL-8/IL-10 Enzyme linked immunosorbent assay (ELISA) ........................................ 35

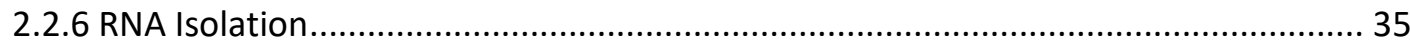

2.2.7 Quantitative PCR of RNA transcripts using SYBR green and probe based method ... 35

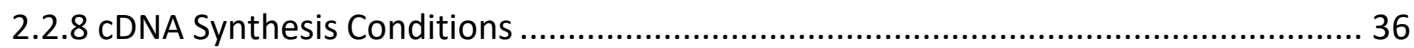

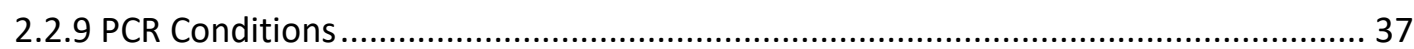

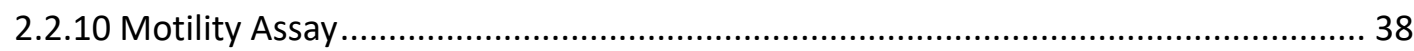

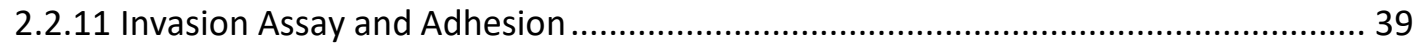

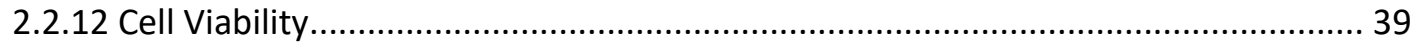

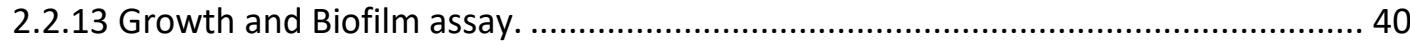

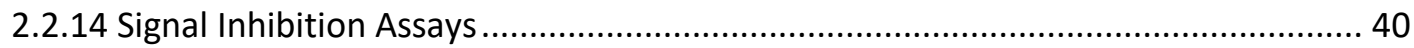

2.3 Bioinformatics, genomics and Campylobacter genomes................................................ 40

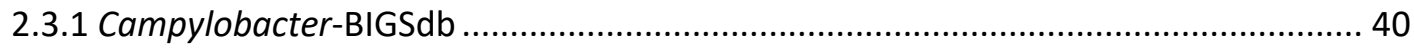

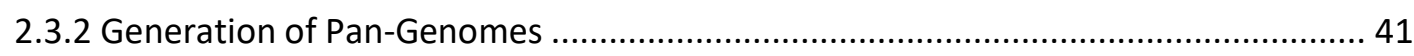

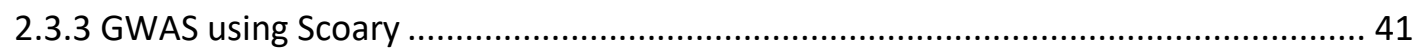

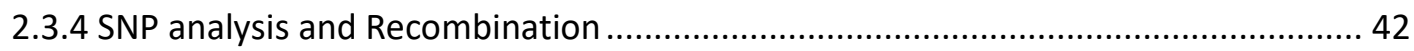

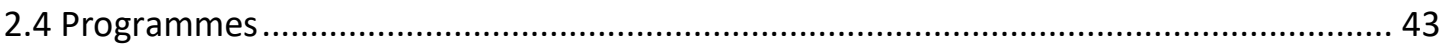

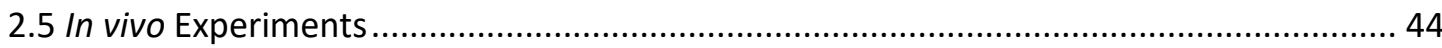

2.5.1 Industrial scale Campylobacter infection study of broiler Chickens .......................... 44

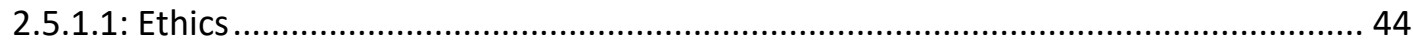

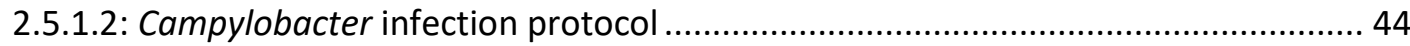

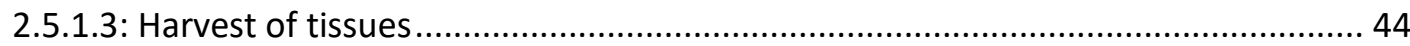

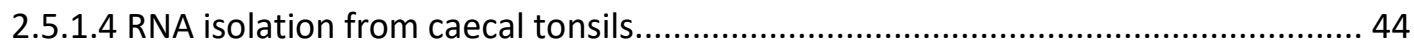

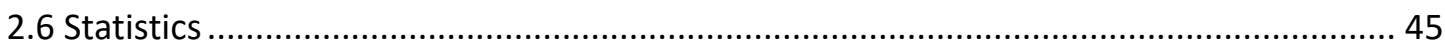

Chapter 3: Characterisation of Campylobacter jejuni strains from four different hosts............ 46

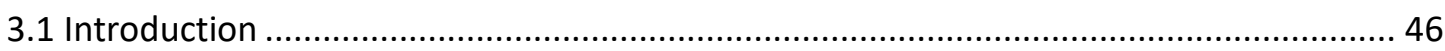

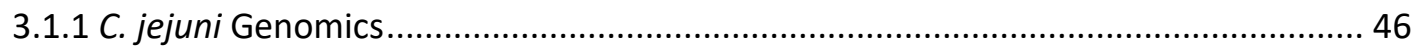




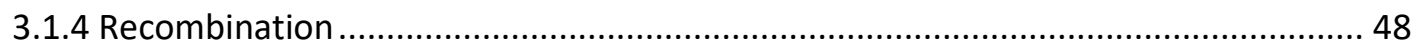

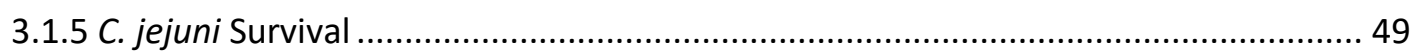

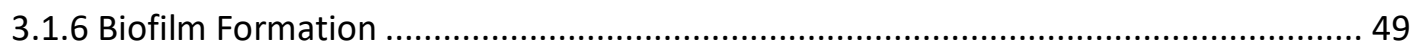

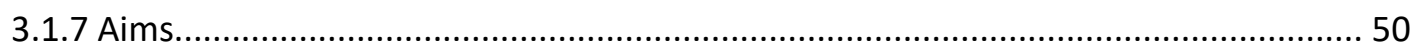

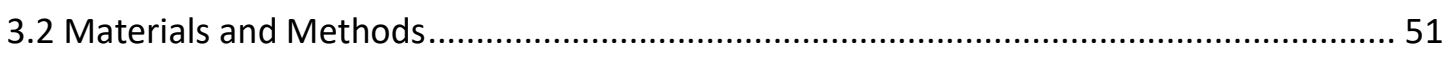

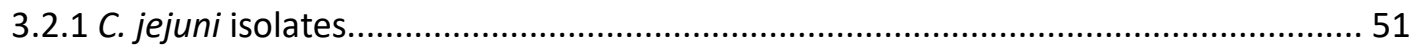

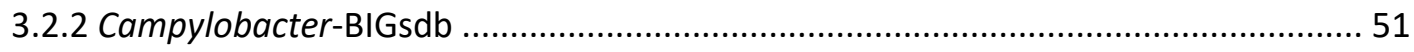

3.2.3 Pan-Genome creation ........................................................................... 51

3.2.4 GWAS using Scoary ............................................................................. 51

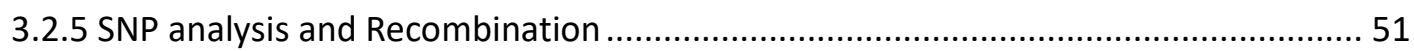

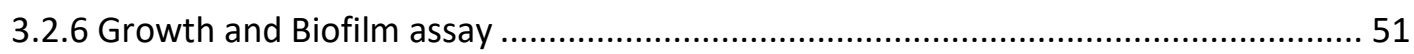

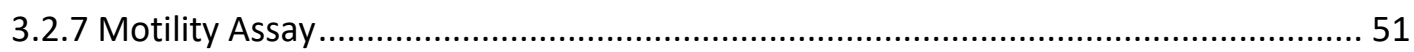

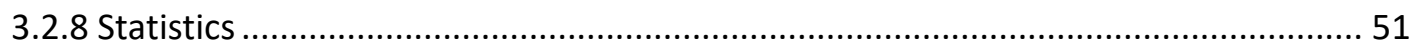

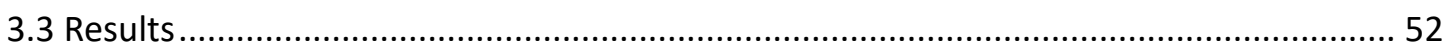

3.3.1 MLST Analysis of $C$. jejuni populations....................................................... 52

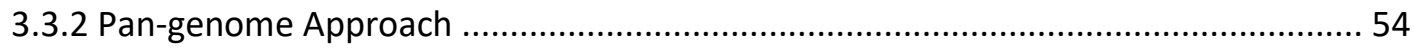

3.3.3 Core vs. Accessory Genome .................................................................... 57

3.3.4 Analysis of $C$. jejuni virulence factors in the accessory genome..........................59

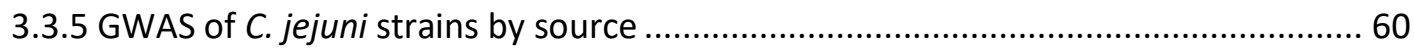

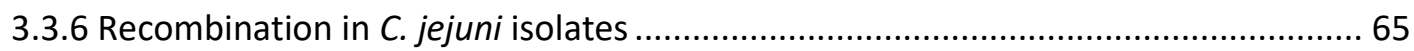

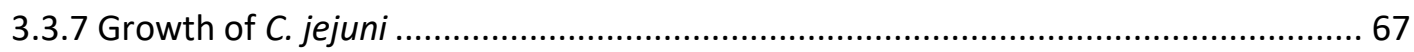

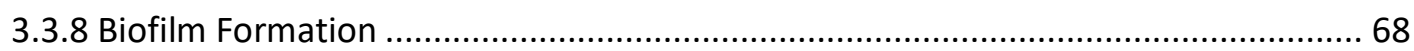

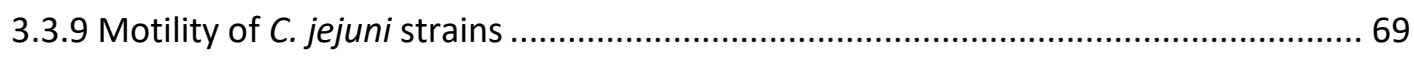

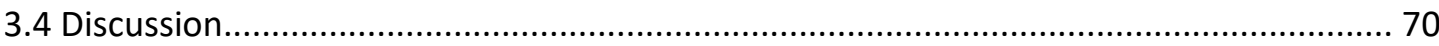

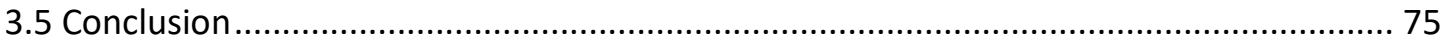

Chapter 4: Diverse responses of Campylobacter jejuni strains in human and chicken intestinal

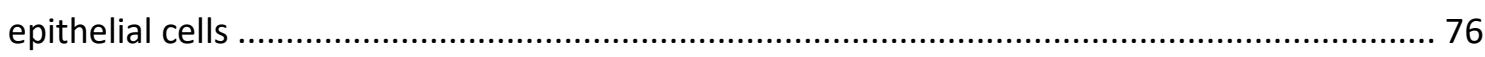

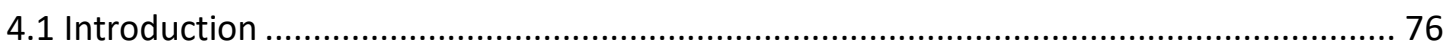

4.1.1 Campylobacter jejuni pathogenesis in humans and chickens ............................. 76

4.1.2 Role of epithelial cells in Campylobacter pathogenesis.................................... 76

4.1.3 Campylobacter induced signalling pathways in epithelial cells ............................ 77

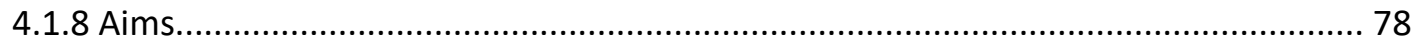

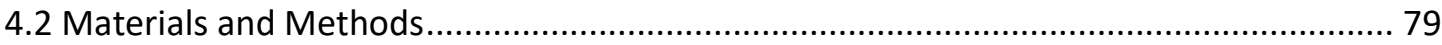


4.2.2 Culture of human and chicken intestinal epithelial cells .................................... 79

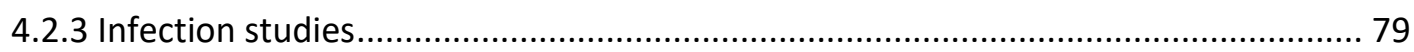

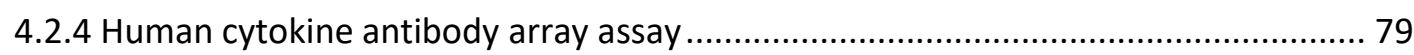

4.2.5 Enzyme linked immunosorbant assay (ELISA)................................................ 80

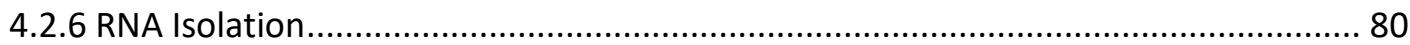

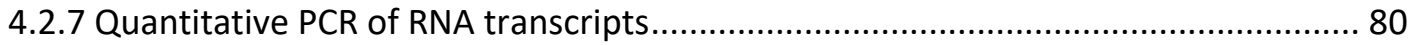

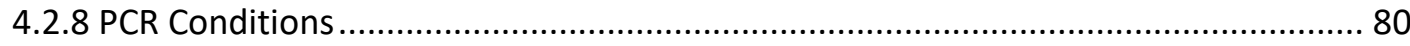

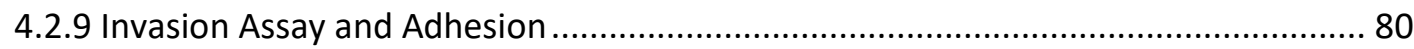

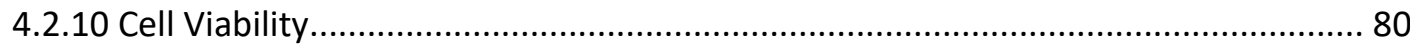

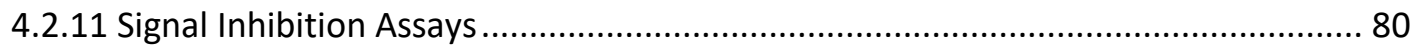

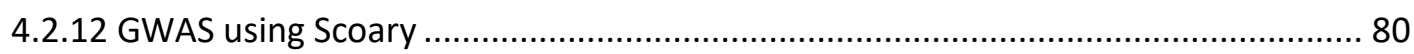

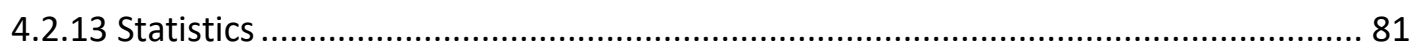

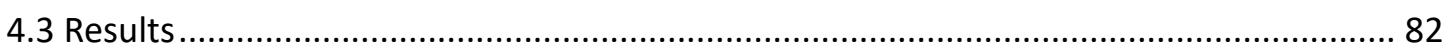

4.3.1 C. jejuni strains used in this study............................................................. 82

4.3.2 Identification of potential immune markers from cytokine panel array assays....... 82

4.3.3 IL-8 secretion from human epithelial cells................................................ 83

4.3.4 Confirmation of the species of the chicken cell line using MHC expression ............ 86

4.3.5 C. jejuni strains produce a large variation in inflammatory cytokine responses ....... 88

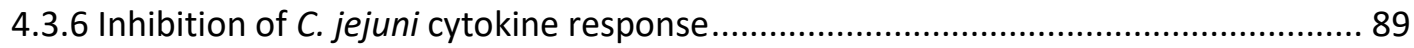

4.3.7 The $c d t A$ gene has an important role in $C$. jejuni-induced epithelial cell toxicity...... 90

4.3.8 Measurement of C. jejuni adhesion and invasion in human and chicken epithelial cells

4.3.9 C. jejuni induced IL-8 production, toxicity and invasion of epithelial cells are more closely correlated in chicken than human cells

4.3.10 Endocytosis of $C$. jejuni is dynamin- and clathrin-dependent in both human and chicken epithelial cells

4.3.11 C. jejuni-induced IL-8 and CXCLi1/2 expression is dynamin- and clathrin-dependent in human and chicken epithelial cells

4.3.12 GWAS analysis of strains with high and low invasion and expression of immune markers

4.4 Discussion

4.5 Conclusion 108

Chapter 5 - Host responses to Campylobacter strains from naturally and experimentally infected chickens 


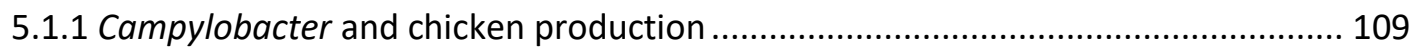

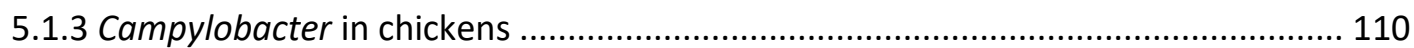

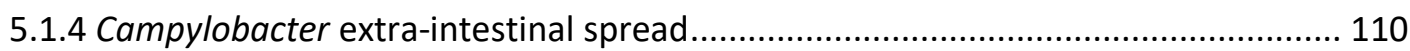

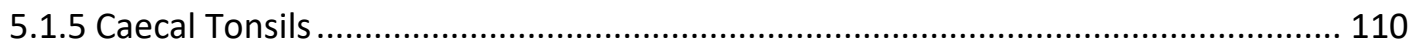

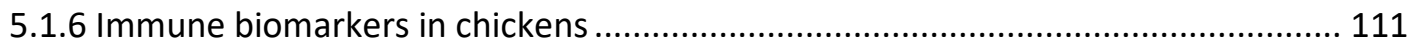

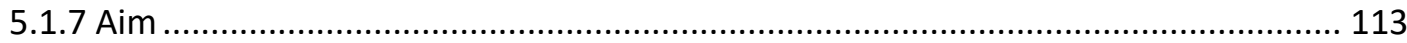

5.2.1 Campylobacter strains from naturally infected chickens .................................... 114

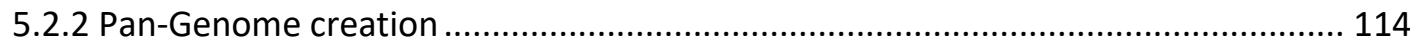

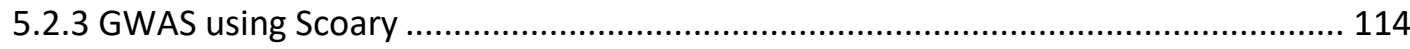

5.2.4 Culture of Human and Chicken Intestinal epithelial cells ..................................... 114

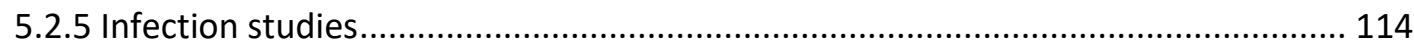

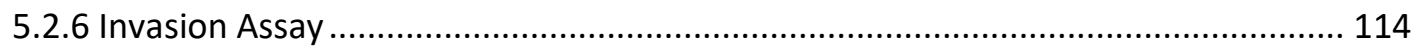

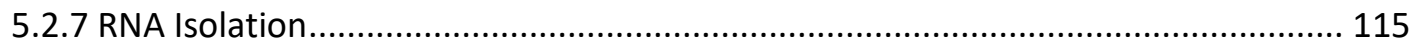

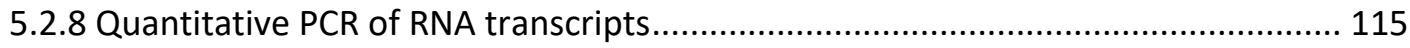

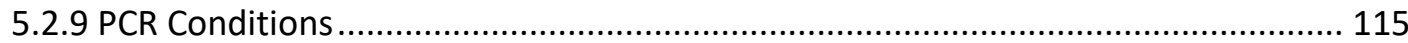

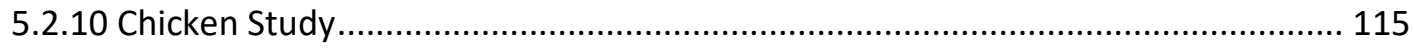

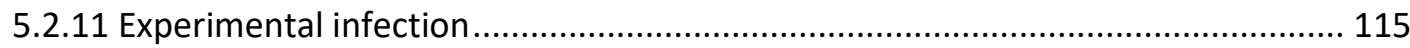

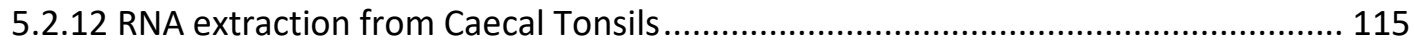

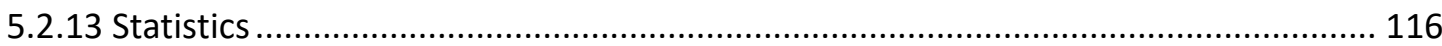

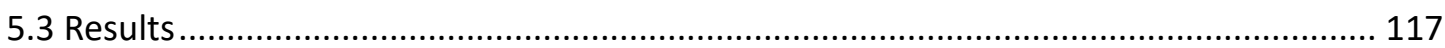

5.3.1 Campylobacter strains from naturally infected chickens ..................................... 117

5.3.2 Genomic analysis of isolates from naturally infected chickens ............................ 117

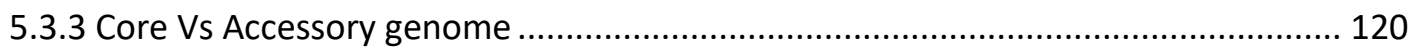

5.3.4 GWAS of isolates from caeca, ileum and liver .................................................. 121

5.3.5 Invasive capability of Campylobacter strains isolated from different sections of the chicken gut and liver from naturally infected chickens ................................................ 124

5.3.6 Analysis of the invasive capability of Campylobacter strains in vitro ..................... 127

5.3.7 In vitro expression of pro-inflammatory cytokines in human and chicken cell lines128

5.3.8 In vitro expression of anti-inflammatory IL-10 in human and chicken cell lines ..... 129

5.3.9 Expression of immune biomarkers in caecal tonsils extracted from Campylobacter-

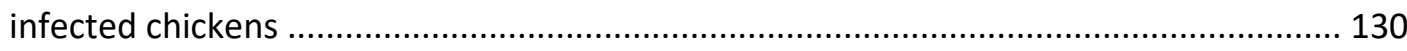

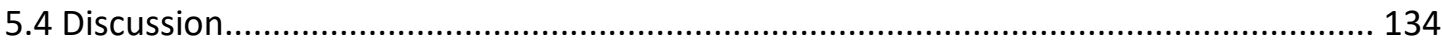

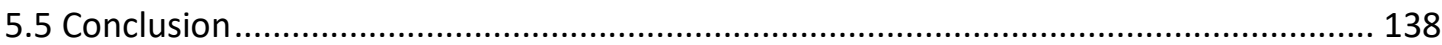




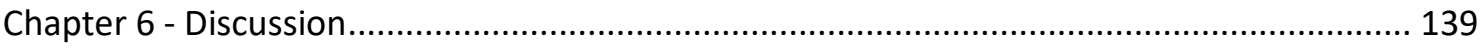

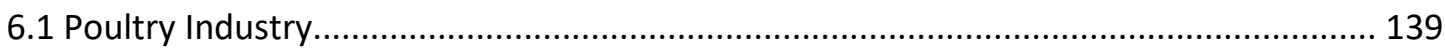

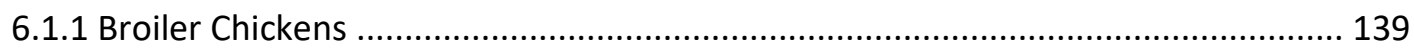

6.1.2 Influence of Campylobacter on the poultry industry.............................................. 140

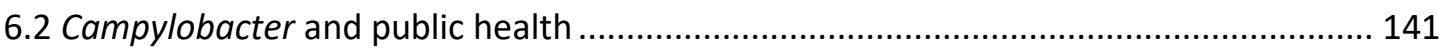

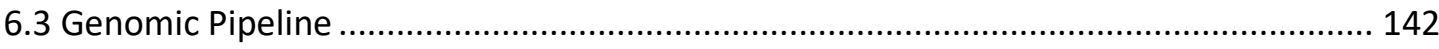

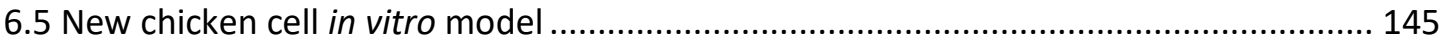

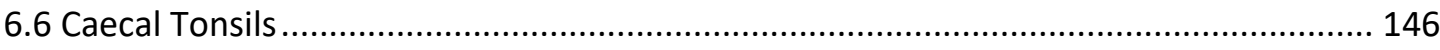

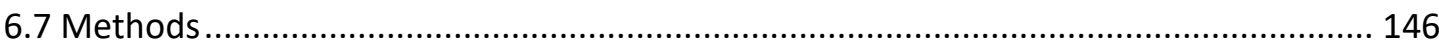

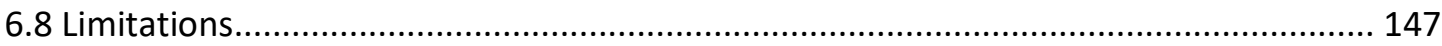

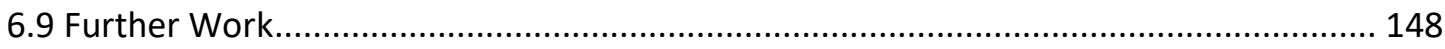

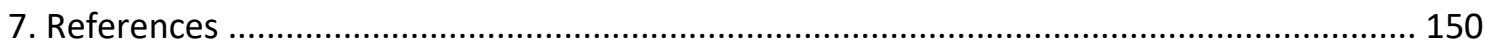

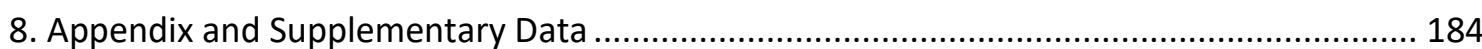




\section{Acknowledgments}

I would first and foremost like to thank Prof. Tom Humphrey and Dr. Tom Wilkinson for the opportunity to study in their lab. This thesis would not have been possible without their continuing support and motivation and I can only apologise for any grey hairs I've caused during my time in Swansea. I would also like to thank Dr. Lisa Williams for all her immense help during my $\mathrm{PhD}$, for her work contributing to chapter 5 and for always being there for a chat and a joke about anything and everything, I definitely owe you a camembert.

I would like to thank everyone in the Microbiology and Infectious Disease group, past, present, and all visiting researchers during my time at Swansea University including; Dr. Llinos Harris, Dr. Susan Murray, Dr. Leonardos Mageiros, Dr. Rhys Jones, Heather Chick, Celeste Felion, Phacharaporn Boonkhot and Nattinee Kittiwan. Thanks to you all for your help and all the laughs along the way.

A huge thank you to all my family, especially Mum, Dad, Matt and Nick, Gran, Granddad and Gramma who have always been there for me and believed in me for as long as I can remember, and of course a thanks to my friends everywhere for keeping me relatively sane throughout the years and who helped me follow my feet so that I could go the distance and change my stars; don't worry, the snow cones are on me.

I would like to dedicate this thesis to my Grandpa, Ivor John, who taught me to be curious and question the world and thanks for all the history stories when growing up. 


\section{List of Tables}

Table 1.1. Factors and genes which have been previously shown to play a role in the adhesion of Campylobacter to the host gut epithelia

Table 1.2. Factors which affect invasion of Campylobacter into epithelial cells and their encoding genes.

Table 1.3. List of TLRs and ligands found in humans and chickens (Kannaki et al., 2010;

Keestra et al., 2013; Owen et al., 2013; Takeda and Akira, 2005)......................................... 21

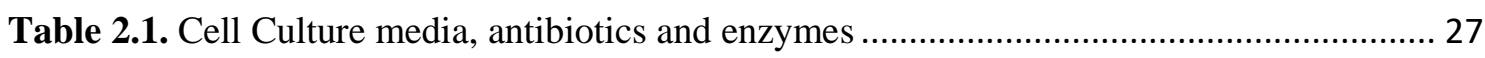

Table 2.2. List of all Campylobacter strains used throughout this thesis.................................. 30

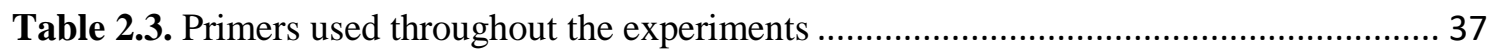

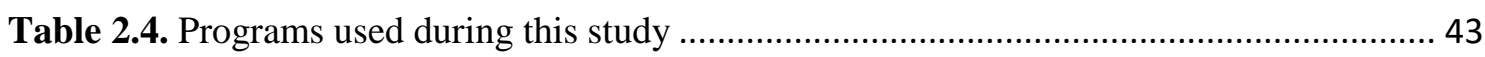

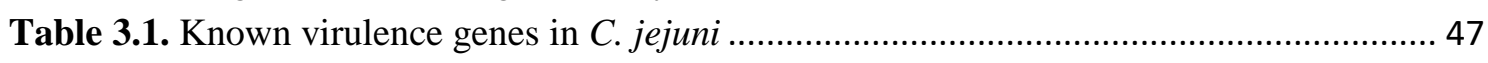

Table 3.2. Presence of known virulence linked genes in the core and accessory genome......... 58

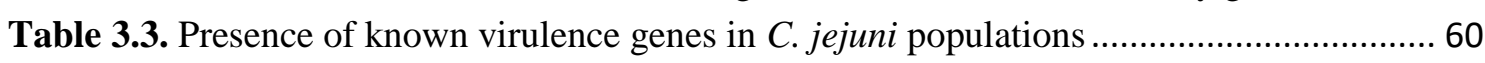

Table 3.4. Top genes found to be associated with strains isolated from humans with the highest

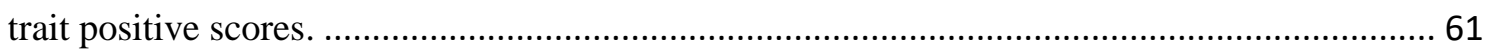

Table 3.5. Top genes found to be associated with strains isolated from chickens with the highest trait positive scores.

Table 3.6. Top genes found to be associated with strains isolated from cattle with the highest trait positive scores.

Table 3.7. Top genes found to be associated with strains isolated from wild birds with the

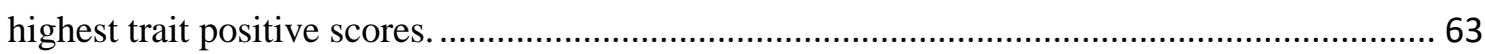

Table 4.1. List of Inhibitors and their respective pathways used during this study. ................. 81

Table 4.14. Genes found to be related to expression of IL-8/CXCLi1/2 ................................ 99

Table 4.15. Genes found to be related to expression of IL-10 ............................................ 100

Table 4.16. Genes found to be related to the level of invasion into both human and chicken

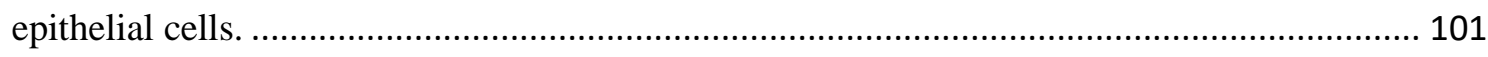

Table 5.1. Presence of known virulence linked genes in the core and accessory genome....... 120

Table 5.2. Top genes found to be related to caeca isolates with the highest trait positive scores.

Table 5.3. Top genes found to be related to ileum isolates with the highest trait positive scores

Table 5.4. Top genes found to be related to liver isolates with the highest trait positive scores 


\section{List of Figures}

Figure 1.1. Source and transmission routes of Campylobacter in human and animal reservoirs. 3 Figure 1.2. Visual representation of the 'zipper' (a) and 'trigger' (b) mechanisms $C$. jejuni uses to

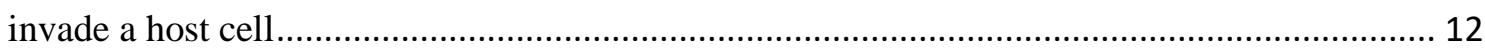

Figure 1.3. Outline of the gastrointestinal tract in humans and chickens ............................... 19

Figure 1.4. TLR Signalling caused by $C$. jejuni in both human and chicken immune systems. 22

Figure 2.1. Genomic pipeline using Prokka, Roary, Scroary and Gubbins to analyse sequences

Figure 3.1. MLST tree of 100 C. jejuni strains labelled by ST (colour) and source (shape).

Human, chicken, cattle and wild bird isolates being represented by a square, circle, triangle and

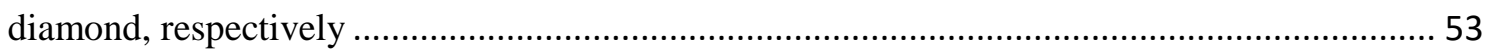

Figure 3.2. Phylogenetic tree of the pan genome of the $C$. jejuni ......................................... 55

Figure 3.3. Representation of the pan-genome showing the core and accessory genome with relation to the phylogenetic tree.

Figure 3.4. Presence of known virulence genes from the accessory genome in human, chicken, cattle and wild bird isolates.

Figure 3.5. Representation of recombination regions present within the 100 Campylobacter genomes analysed. Red regions represent recombination regions shared with two or more isolates and blue regions denote unique recombination regions to an individual isolate....

Figure 3.6. Growth of $C$. jejuni $(\mathrm{n}=100)$ strains under varying conditions. a) Strains subjected to a microaerophilic environment at $37^{\circ} \mathrm{C}$ and $42^{\circ} \mathrm{C}$; b) strains subjected to normal atmospheric conditions at $37^{\circ} \mathrm{C}$ and $42^{\circ} \mathrm{C}$.

Figure 3.7. Effect of growth atmosphere and temperature on $C$. jejuni biofilm formation. C. jejuni strains were grown at 37 and $42^{\circ} \mathrm{C}$ under microaerophilic conditions (a) and under normal atmospheric conditions (b), with biofilm organised by source of C. jejuni strain at 42 (c) and 37 ${ }^{\circ} \mathrm{C}(\mathrm{d})$.

Figure 3.8. Motility assay performed on a $25 \%$ sub-group of $C$. jejuni isolates from different sources (a) and the presence of flaA and flaB genes (b). Isolates coloured green represent isolates which are flaA/B positive.

Figure 4.1. Interaction of Campylobacter with host epithelial cells in human and chicken derived cells

Figure 4.2. Human cytokine array assay performed on uninfected cells, cell infected by 11168 and cells infected by M1. Red box indicates the IL-8 cytokine expression in 11168 and M1; blue box indicates the control wells (a), with markers with the overall largest mean pixel density in the infected samples plotted (b).

Figure 4.3 Mean IL-8 secretion induced by $C$. jejuni at (a) over time, (b) $4 \mathrm{~h}$ of infection, (c) $6 \mathrm{~h}$ of infection and (d) 24h of infection categorised by source of Campylobacter strain and d) Mean IL-8 secretion induced by $C$. jejuni at $24 \mathrm{~h}$, categorised by source of Campylobacter strain...... 85

Figure 4.4. Mean IL-8 secretion induced by $C$. jejuni at (a) $4 \mathrm{~h}$ of infection (b) $6 \mathrm{~h}$ of infection (c) $24 \mathrm{~h}$ of infection categorised by sequence type the of Campylobacter strain 86

Figure 4.5. Expression of MHC induced by C.jejuni after 24h of infection on (a) HT-29 and $8 \mathrm{E} 11$ cell lines; with $(\mathrm{b}, \mathrm{c})$ the breakdown of responses by source and $(\mathrm{d}, \mathrm{e})$ by sequence type of Campylobacter.

Figure 4.6 Expression of pro- and anti- inflammatory cytokines and chemokine's in HT-29 human and $8 \mathrm{E} 11$ chicken epithelial cells after infection with $C$. jejuni for 24 hours before 
isolation of total RNA and quantification of specific mRNA by qPCR. (a) Expression of IL8/CXCLi1/CXCLi2 in both HT-29 and 8E11 cell lines (b) Expression of IL-10 in HT-29 and $8 \mathrm{E} 11$ cell lines

Figure 4.7. Prior to infection with $C$. jejuni, cells were incubated with signalling inhibitors for up to 30 minutes. Then specific mRNA for IL-8 / CXCLi1 / CXCLi2 (a, b, c) and IL-10 (d, e) were measured. Each dot represents three biological replicates in one strain. Results are also expressed as mean +/- SD of all strains measured. Differences were considered significant if $\mathrm{p} \leq 0.05$.

Figure 4.8. Reduction of cell viability after infection with $C$. jejuni. HT-29 and 8E11 cells were infected with $C$. jejuni for 24 hours before isolation of cell supernatants for alamar blue viability assay. (a) Toxicity responses in HT-29 and 8E11 epithelial cells. (b and c) Toxicity responses organised by the presence and absence of the cdtA gene. Each dot represents three biological replicates in one strain. Results are also expressed as mean +/- SD of all strains measured. Differences were considered significant if $\mathrm{p} \leq 0.05$.

Figure 4.9 HT-29 and 8E11 cells were infected with $C$. jejuni for 6 hours before isolation and quantification of adherent and invasive $C$. jejuni. (a) Adhesion of $C$. jejuni strains to HT-29 and 8E11 cells. (b) Invasion of $C$. jejuni into HT-29 and 8E11 cells. (c,d) Treatment of HT-29 and $8 \mathrm{E} 11$ cells with signalling inhibitors for 30 minutes prior to invasion assays. Each dot represents three biological replicates in one strain. Results are also expressed as mean +/- SD of all strains measured. Differences were considered significant if $\mathrm{p} \leq 0.05$.

Figure 4.10. Relationships between $C$.jejuni-induced cellular invasion, toxicity and cytokine production; phenotypic data was subjected to regression analysis. (a) IL-8 (b) CXCLiland (c) CXCLi2 correlations with $C$. jejuni invasion. (d) IL-8, (e) CXCLi1, (f) CXCLi2 correlations with toxicity. (g) HT29 cell viability correlation with invasion (h) 8E11 cell viability correlation with invasion.

Figure 4.11 The effect of inhibition of cellular endocytosis on $C$. jejuni invasion into epithelial cells; (a) HT-29 and (b) 8E11 cells were pre-treated with endocytosis inhibitors prior to infection with $C$. jejuni for 6 hours and then $C$. jejuni invasion was assessed. Each dot represents three biological replicates in one strain. Results are also expressed as mean +/- SD of all strains measured. Differences were considered significant if $\mathrm{p} \leq 0.05$.

Figure 4.12 The effect of inhibition of reduction of cell viability of epithelial cells after cultured with C. jejuni; (a) HT-29 and (b) 8E11 cells were pre-treated with endocytosis inhibitors prior to infection with C. jejuni for 6 hours and then $C$. jejuni invasion was assessed. Each dot represents three biological replicates in one strain. Results are also expressed as mean +/- SD of all strains measured. Differences were considered significant if $\mathrm{p} \leq 0.05$.

Figure 4.13 The effect of inhibition of endocytosis of cytokines cytokine expression in epithelial cell;. (a) HT-29 and (b, c) 8E11 cells were treated with endocytosis inhibitors prior to infection with $C$. jejuni for 24 hours. Then total RNA was isolated and specific mRNA was quantified by qPCR. Expression of (a) IL-8, (b) CXCLi1 and (c) CXCLi2 in epithelial cells. Each dot represents three biological replicates in one strain. Results are also expressed as mean +/- SD of all strains measured. Differences were considered significant if $\mathrm{p} \leq 0.05$.

Figure 4.14 Identified aspects of the chicken system during this chapter............................... 105

Figure 4.15 Preliminary TLR expression in human and chicken cell lines ............................ 106

Figure 5.1. Maximum likelihood tree of the pan genome of Campylobacter isolates from naturally infected chickens

Figure 5.2. Representation of the pan-genome displaying the core and accessory genome with relation the phylogenetic tree. The core genome is made up of the core genes (present in 99- 
$100 \%$ of isolates) and soft core ones (present in $95-99 \%$ of isolates), with the accessory genome comprising shell genes (present in $15-95 \%$ of isolates) and the cloud genes (present in 0-15\% of isolates).

Figure 5.3. Gentamicin protection assay of 42 Campylobacter strains from caeca, ileum and liver in 8E11 and Caco-2 cells (a), with comparison of strains from the (b) caeca, (c), ileum and (d) liver in both cell lines, with the breakdown of invasive responses by group in (e) 8E11 and

(f) $\mathrm{Caco}-2$ 125

Figure 5.4. Gentamicin protection assay results with strains isolated from the same bird joined across anatomical location (a) 8E11 and (b) Caco-2 cells. 126

Figure 5.5. Gentamicin protection assay performed on three cell lines, HT-29, 8E11 and Caco-2 displaying overall invasive capability of the strains (a) with each individual strain marked through each cell line (b). Invasion with all six strains combined can be seen in grey. 127

Figure 5.6. Expression of IL-8 and CXCLi1/2 in the three cell lines tested; HT-29, 8E11 and Caco-2 (a), with each individual isolate linked (b). Invasion with all six strains combined can be seen in grey.

Figure 5.7. Expression of IL-10 in the three cell lines tested; HT-29, 8E11 and Caco-2 (a), with each individual isolate linked (b). Invasion with all six strains combined can be seen in grey.129 Figure 5.8 Expression of a variety of $T_{h} 1 / T_{h} 2, T_{h 17}$ and $T_{h} 22$ markers in caecal tonsils extracted from uninfected chicken, chickens that were infected and kept in chicken sheds and chickens who were infected and put through the industry production chain including being handled, transportation and gas/stun killed. Immune markers measured were (a) IL-8, (b) IL-10, (c) IL18, (d) TNF $\alpha$, (e) IFN $\gamma$, (f) IL-6, (g) IL-1 $\beta$ and (h) IL-17.

Figure 5.9. Correlations of Campylobacter counts in the caeca compared to expression of cytokines. Immune markers measured were (a) IL-8, (b) IL-10, (c) IL-18, (d) TNF $\alpha$, (e) IFN $\gamma$, (f) IL-6, (g) IL-1 $\beta$ and (h) IL-17

Figure 5.10. Correlations of Campylobacter counts in the ileum compared to expression of cytokines. Immune markers measured were (a) IL-8, (b) IL-10, (c) IL-18, (d) TNFa, (e) IFN (f) IL-6, (g) IL-1 $\beta$ and (h) IL-17 
Abbreviations

\begin{tabular}{|c|c|}
\hline AMP & adenosine monophosphate \\
\hline AJs & adherens junctions \\
\hline BIGsdb & bacterial isolate genome sequence database \\
\hline Caco-2 & heterogeneous human epithelial colorectal adenocarcinoma cell line \\
\hline CBA & Columbian blood agar \\
\hline CCDA & Campylobacter blood-free selective medium \\
\hline C. concisus & Campylobacter concisus \\
\hline C. coli & Campylobacter coli \\
\hline C. curvus & Campylobacter curvus \\
\hline C. fetus & Campylobacter fetus \\
\hline C. jejuni & Campylobacter jejuni \\
\hline cDNA & complementary deoxyribonucleic acid \\
\hline CDS & coding sequence \\
\hline $\mathrm{CFU}$ & colony forming units \\
\hline CDT & cytolethal distending toxin \\
\hline CT & cycle threshold \\
\hline DAMPs & damage-associated molecular patterns \\
\hline DMEM F-12 & Dulbecco's modified eagle medum: nutrient mixture F-12 \\
\hline DNA & deoxyribonucleic acid \\
\hline ELISA & enzyme-linked immunosorbent assay \\
\hline FAE & follicle-associated epithelium \\
\hline FBS & fetal bovine serum \\
\hline GALT & gut-associated lymphoid tissue \\
\hline GC-content & guanine-cytosine content \\
\hline GCPs & G-protein coupled receptors \\
\hline GI & gastrointestinal \\
\hline GTP & guanosine triphosphate \\
\hline GWAS & genome-wide association study \\
\hline HD11 & Chicken macrophage cell line \\
\hline HT-29 & Homo sapiens colon colorectal adenocarcinoma cell line \\
\hline $\operatorname{Ig}$ & immunoglobulin \\
\hline IL & interleukin \\
\hline IBD & inflammatory bowel disease \\
\hline IBS & irritable bowel syndrome \\
\hline IEL & intra-epithelial lymphocytes \\
\hline IECs & intestinal epithelial cells \\
\hline IFN & interferon \\
\hline IRAK & interleukin-1 receptor-associated kinase \\
\hline IKK & ІкВ kinase complex \\
\hline LOS & lipooligosaccharides \\
\hline
\end{tabular}




$\begin{array}{ll}\text { LPS } & \text { lipopolysaccharides } \\ \text { MALT } & \text { mucosa-associated lymphoid tissue } \\ \text { MAPK } & \text { Mitogen-activated protein kinase } \\ \text { MH } & \text { Muller Hinton } \\ \text { MLST } & \text { Multi-locus sequence typing } \\ \text { MyD88 } & \text { myeloid differentiation factor } 88 \\ \text { NF-KB } & \text { nuclear factor kappa-light-chain-enhancer of activated B } \\ \text { OD } & \text { Optical density } \\ \text { PAMPs } & \text { pathogen-associated molecular patterns } \\ \text { PBS } & \text { phosphate-buffered saline } \\ \text { PCR } & \text { polymerase chain reaction } \\ \text { PFGE } & \text { pulsed field gel electrophoresis } \\ \text { PMA } & \text { phorbol 12-myristate 13-acetate } \\ \text { P/S } & \text { penicillin / streptomycin } \\ \text { RNA } & \text { ribonucleic acid } \\ \text { PRRs } & \text { pattern recognition receptors } \\ \text { r/m } & \text { recombination relative to mutation } \\ \text { RT } & \text { reverse transcription } \\ \text { ROS } & \text { reactive oxygen species } \\ \text { SEM } & \text { scanning electron microscope } \\ \text { SNP } & \text { single-nucleotide polymorphism } \\ \text { Spp. } & \text { species } \\ \text { ST } & \text { sequence type } \\ \text { TH } & \text { T helper cell } \\ \text { T3SS } & \text { type III secretion system } \\ \text { T4SS } & \text { type IV secretion system } \\ \text { TRAF } & \text { TNF receptor associated factor } \\ \text { TNF } & \text { tumour necrosis factor } \\ \text { TIR } & \text { toll/interleukin-1 receptor } \\ \text { TLR } & \text { toll-like receptor } \\ \text { TJs } & \text { tight junctions } \\ \text { TRIF } & \text { TIR-domain-containing adaptor-inducing IFN- } \beta \text { factor } \\ \text { UK } & \text { United Kingdom } \\ \text { VBNC } & \text { viable but nonculturable } \\ \text { WGS } & \text { whole genome sequencing } \\ & \end{array}$




\section{Chapter 1: Introduction}

\subsection{History of Campylobacter}

Campylobacter was first described in 1886 as a Vibrio-like organism in stool samples of infants by Theodor Escherich, who observed and described non-culturable spiral-shaped bacteria (Silva et al., 2011). Following this, in 1906, Vibrio-like organisms were identified by two British veterinarians who described the presence of a 'large number of a peculiar organism' in the uterine mucus of a pregnant sheep (Zilbauer et al., 2008). In 1913, McFadyean and Stockman isolated microorganisms from aborted bovine foetuses (Skirrow, 2006), and in 1927 Smith and Orcutt named a group of bacteria Vibrio jejuni; these were isolated from the faeces of cattle suffering from diarrhoea. Doyle in 1944, isolated a different 'Vibrio' from the faeces of pigs and classified the bacteria as Vibrio coli (Doyle, 1944; Vandamme et al., 2010). The genus Campylobacter was first proposed in 1963 by Sebald and Véron due to the low guanine-cytosine content (GCcontent)of the bacteria, their microaerobic growth requirement and their nonfermentative metabolism (On, 2001). It took until 1973, for Belgium scientists to first describe the isolation of Campylobacter from human diarrhoeastool samples (Butzler et al., 1973). Later, Campylobacter spp. were established as a common human pathogens (Altekruse et al., 1999; Skirrow, 1977).

\subsection{Campylobacter}

Campylobacter refers to a genus of microaerophilic, cytochrome oxidase positive, curved Gram-negative rods, which exhibit a characteristic corkscrew motility. There are currently 17 species and 6 sub-species within the genus (Parte, 2014).

Campylobacter is the leading cause of bacterial food-borne diarrhoal disease worldwide, with symptoms ranging from mild diarrhoea to more serious infections that can result in chronic conditions such as reactive arthritis and neurological problems; especially in elderly people (Silva et al., 2011). It is frequently found in poultry, particularly in chicken, with the result that chicken-associated Campylobacter is a major cause of foodborne infection (PHE, 2017). In the UK alone, an estimated 700,000 cases of infection and more than 100 deaths can be attributed to Campylobacter annually (PHE, 2017). The cost to the UK economy is estimated to be $£ 900$ million (DEFRA, 2012) but this may well be an underestimation, due to under-reporting of cases. In comparison, there were 8,630 confirmed laboratory cases of Salmonella in England and Wales in 2016 (PHE, 2018). In the developed world, there are two main species that are 
primarily responsible for human cases of Campylobacter, which are also detected in poultry at retail sale (Silva et al., 2011). These are Campylobacter jejuni and Campylobacter coli. However, Campylobacter upsaliensis is also important in developing countries (Parkhill et al., 2000).

\subsection{Campylobacteriosis}

\subsubsection{Human infections}

Campylobacter infection of humans is termed campylobacteriosis; with C. jejuni being the most commonly isolated species from stool samples (WHO, 2012). Campylobacteriosis is one of the most common bacterial infections in humans and is primarily attributed to a foodborne source. Each year almost 1 in 10 people fall ill from foodborne diseases which can be severe; especially in the young and elderly (WHO, 2012). Campylobacter is 1 of 4 key global causes of diarrhoeal diseases, with 550 million people contracting the infection annually (WHO, 2012). It is estimated that $1 \%$ of the EU population is infected each year. Human infections can come from several different sources; the most frequently reported is from contaminated chicken meat, as discussed above. However, cattle and sheep faeces and carcasses, wild birds and environmental waters (Figure 1.1) have been attributed as sources of human disease (Dasti et al., 2010; Kaakoush et al., 2015).

The onset of campylobacteriosis symptoms occur usually within 2 to 5 days after infection with the bacteria, but the incubation period can sometimes range from 1 to 10 days; with the most common clinical symptoms being diarrhoea, severe abdominal pain, high fever, headache and nausea. Symptoms can last from 3 to 6 days, with most cases being self-limiting. While death from campylobacteriosis is not common; young children, elderly and immunocompromised patients, such as those suffering from AIDS, are the most at risk (European Food Standards Agency, 2018; WHO, 2018). Campylobacteriosis can sometimes cause complications such as bacteraemia, hepatitis, pancreatitis and miscarriage with various degrees of frequency. Post-infection complications have also been reported which include; reactive arthritis and neurological disorders such as Guillain-Barré syndrome (European Food Standards Agency and Agency, 2014; WHO, 2012). 


\subsubsection{Chickens and Campylobacter}

Campylobacter jejuni strains can cause disease in chickens and Ruiz-Palacios et al., (1981) were the first to show that the bacterium causes diarrhoea in these animals. Sanyal et al ., (1984) also demonstrated that $C$. jejuni causes diarrhoea in infant chickens and that damage to the gut mucosa resulted in extra-intestinal spread of the bacteria to the blood, spleen and liver. Chickens infected with Campylobacter have been shown to have poorer performance than non-infected animals and can develop hock marks and pododermatitis, indicators of poor gut health (Humphrey et al., 2014; Ruizpalacios et al., 1981; Williams et al., 2013). Inflammation and damage of the gut mucosa have also been observed in infected chickens (Awad et al 2014; Humphrey et al., 2014). 


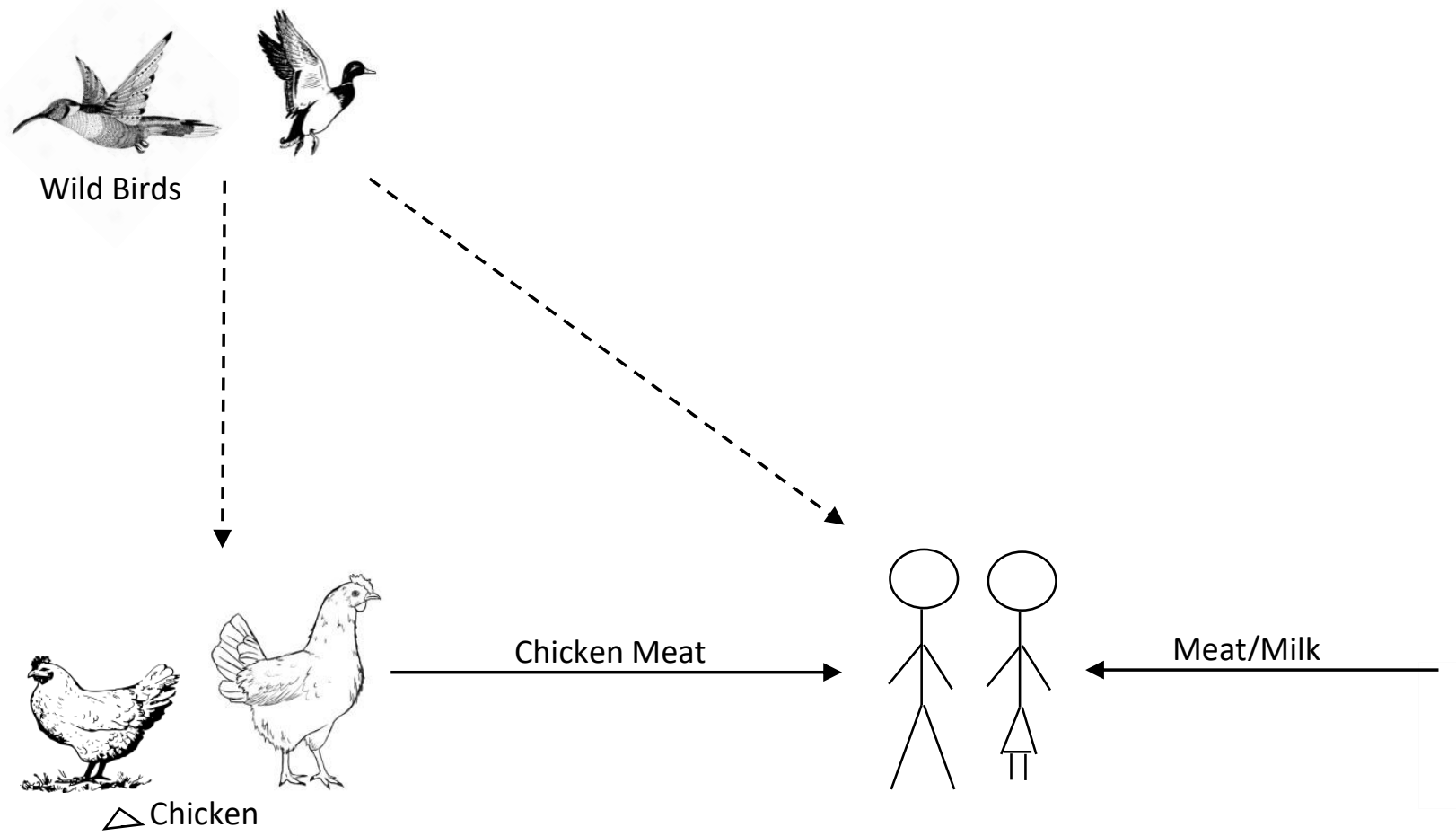

Figure 1.1. Source and transmission routes of Campylobacter in human and animal reservoirs with to poultry and cattle with rarer transmission from wild birds. 


\subsection{Campylobacter in different hosts}

Many species of Campylobacter have been observed to be specific to one host, such as Campylobacter rectus isolated only from humans and Campylobacter mucosalis which is found in pigs (Rams et al., 1993; Roop et al., 1985; van der Walt et al., 1988). In contrast, other species such as $C$. jejuni and $C$. coli, have been isolated from many different hosts (Figure 1.1) including: humans, chickens, cattle, wild birds and many different water sources such as groundwater, sewage and untreated drinking water, suggesting a more generalist nature (Ogden et al., 2009; Pielsticker et al., 2012). The advancement in genomic techniques over the past decade has led to the characterisation of thousands of Campylobacter isolates using Multilocus sequence typing (MLST) which has identified clonal complexes and sequence types (STs) that are usually only sampled from the gut of a single host species and can be termed 'host specialists'; which include 'chicken specialists' such as STs-661, -573, -574, -607, -443, -460, -283 and ST-257 and 'cattle specialists' such as STs-61 and -42 (Dearlove et al., 2016; Pascoe et al., 2015). Other STs, such as -45 and -21 clonal complexes have been found in multiple host species and can be termed 'host generalists' (Sheppard et al., 2011). Analysis of Campylobacter populations show that certain clusters may be differentiated by their host sources (Colles et al., 2008; McCarthy et al., 2007; Sheppard et al., 2010b, 2010a).

\subsection{Campylobacter Genomics}

Campylobacter jejuni strain 11168 was the first sequenced in 2000 by the Sanger Centre and this showed that it had 1,641,481 base pairs, containing 1,654 protein coding genes. Sequencing of this $C$. jejuni also revealed that the bacterium has hypervariable sequences, which may be important as a survival strategy (Parkhill et al., 2000).

Campylobacter coli and C. jejuni have naturally high transformation rates of 1/1000 and 1/10,000 bacterial cells respectively (Wang and Taylor, 1990). This ability to incorporate foreign DNA into the genome has a major influence on the population genomics and evolution of the species (Wilson et al., 2009). MLST data have shown that Campylobacter populations are subject to high levels of horizontal gene transfer within and between species and they display partial clonality, where strains with similar genotypes form clusters, known as clonal complexes, which contain related sequence types (STs) (Sheppard et al., 2008; Smith et al., 1993). 
Campylobacter populations are highly structured into clusters of related lineages, which include deep branching clades, with regards to $C$. coli, and clonal complexes of isolates that share four or more alleles at seven MLST loci (Dingle et al., 2001; Sheppard et al., 2008).

\subsection{Isolation and identification of Campylobacter}

Campylobacter can be isolated by direct culture onto selective agars or put through an enrichment process and then plated onto a selective medium such as blood-free selective medium (CCDA) and incubated microaerobically for $24-48 \mathrm{~h}$ at $42^{\circ} \mathrm{C}$. Colonies on the selective agar are scrutinised for the characteristic moist translucent appearance, Gram stained and confirmed further through a positive oxidase test (Health Protection Agency, 2013) and a lack of growth in air on blood agar.

Further identification of Campylobacter can also be undertaken using established molecular methods such as pulsed field gel electrophoresis (PFGE) and MLST together with whole genome sequencing (WGS) (Health Protection Agency, 2013). PFGE can detect variation between different strains using rare-cutting restriction endonucleases and the resultant fragments can then be separated based on their size on an agarose gel. PFGE can be used to characterise isolates related epidemiologically from an outbreak due to its nature of being highly discriminatory (Ribot et al., 2001). PFGE has been used successfully to identify Campylobacter and characterise subtypes of $C$. jejuni (Ribot et al., 2001).

MLST determines variations in DNA sequences in a set of housekeeping genes and characterises strains by their unique allelic profiles. The housekeeping genes used for MLST for C. jejuni and C. coli are aspA, glnA, gltA, pgm, tkt and uncA which encode for aspartate, ammonia-lyase, glutamine synthetase, citrate synthase, 2,3bisphosphoglycerate-independent phophoglycerate mutase, transketolase and ATP synthase alpha subunit respectively (Dingle et al., 2001). MLST involves PCR amplification followed by DNA sequencing, which yields nucleotide differences between strains, which can be checked at a variable number of genes. MLST detects all the nucleotide polymorphisms within a gene rather than just non-synonymous changes that alter the electrophoretic mobility of the protein product and thus is a highly discriminatory technique. MLST has been used successfully to differentiate strains and identify clonal lineages of Campylobacter species; such as C. jejuni, C. coli, C. fetus 
and emerging species such as $C$. curvus and C. concisus (Dingle et al., 2005; Miller et al., 2012). MLST comes with disadvantages for laboratory testing, as there are substantial costs and work required to amplify, determine and proof read the nucleotide sequence of the target DNA fragments (Dingle et al., 2001, 2005; Health Protection Agency, 2013).

WGS is a method by which the complete DNA sequence of an organism's genome is determined and has been used to sequence the complete genome of $C$. jejuni (Parkhill et al., 2000). The process of WGS sequences all chromosomal DNA as well as that contained in plasminds (Parkhill et al., 2000). WGS has been suggested to be able to connect strains epidemiologically from the same infection source despite the incident being identified originally as a sporadic infection (Kovanen et al., 2014). Advances in sequencing technology have led to lower WGS costs and the ability to produce large volumes of sequence data. WGS can identify a broad range a variants and can identify SNPs, stuructural variants, copy number variants and repeat expansion which yields more accurate sequence information (Heather and Chain, 2016; Levy and Myers, 2016; Schwarze et al., 2018).

\subsection{Virulence}

C. jejuni has six important gene functions that determine its pathogenic potential, including: i) motility, ii) attachment, iii) invasion and internalisation, iv) intracellular survival, v) metabolic and environmental regulation, vi) endotoxin and exotoxin production (Bolton, 2015).

\subsubsection{Motility}

C. jejuni is a highly motile organism which utilizes unipolar and bipolar flagella, which are very important for infection/colonisation in humans and other animals (Guerry, 2007). This process is regulated by a complex chemotaxis system that allows the organism to swim toward attractants and away from repellents (Lertsethtakarn et al., 2011).

Type III secretion systems (T3SS) are complex macromolecular structures which allow gram-negative bacteria to secrete proteins across the inner and outer membranes without a periplasmic intermediate. Classical T3SS in bacteria such as Salmonella allow the bacteria to deliver effector proteins from the bacteria cytosol directly to the cytosol of a target cell. These delivered effector proteins modulate host cell behaviour to facilitate 
uptake of the bacteria (Neal-McKinney and Konkel, 2012). The classical T3SS are comprised of a protein complex, which spans both bacterial membranes, which is attached to a needle complex that extends from the cell. The needle complex contains translocon proteins in a hollow conduit at the tip which incorporate into the membrane of the host cell, thus allowing for delivery of effector proteins into the host cytosol (Guerry, 2007; Neal-McKinney and Konkel, 2012).

The flagellum in Campylobacter, which is a T3SS with components homologous to the classical T3SS, is comprised of a basal body, hook and filament (Neal-McKinney and Konkel, 2012). The basal body spans both bacterial membranes attached to the hook which is a curved conduit. The hook is composed primarily of the protein FlgE with hook-filament proteins FlgK and FlgL at its cap. The flagellar T3SS can change its export specificity to allow secretion of FlaA and FlaB which go on to form the flagellar filament (Guerry, 2007; Lertsethtakarn et al., 2011; Matsunami et al., 2016; NealMcKinney and Konkel, 2012; Samuelson et al., 2013). The flagella can also function to secrete non-flagellar proteins which help module virulence such as CiaC (NealMcKinney and Konkel, 2012; Poly, 2008).

The flagella of Campylobacter are composed of major and minor flagellin sub-units, FlaA and FlaB, which are regulated by $\sigma^{28}$ dependent and $\sigma^{54}$-dependent promoters, respectively (Guerry, 2007). Expression of flagella in $C$. jejuni is mediated by FlgS, a $\sigma^{54}$ sensor kinase and FlgR, a $\sigma^{54}$ response regulator (Hendrixson and Dirita, 2003).

Another protein, FlaC, is highly conserved among C. jejuni strains and its gene was thought originally to be a third flagellin based on amino acid carboxy region similarities with FlaA and FlaB (Faber et al., 2015; Guerry, 2007). FlaC also requires a flagellar structure to be present before it can be secreted and has been proposed to act as a secreted effector protein which modulates the immune response in the intestinal tract and may contribute to the persistence of the Campylobacter in the gut (Faber et al., 2015). The two other flagellin genes, fla $A$ and flaB, are arranged in tandem. The flaA gene is essential for adherance and invasion of epithelial cells, and colonisation/infection of the gastrointestinal tract (Jain et al., 2008). C. jejuni possesses a flagellum that is made up of an O-linked glycosylated flagellin; a two component system compromising the sensor FlgS and the response regulator FlgR which is central for the regulation of the Campylobacter flagellum (Silva et al., 2011). 
Campylobacter flagellar are heavily glycosylated up to 19 times, with changes in the glycan composition affecting the autoaggulation and microcolony formation on the intestinal epthelial cells which contribute to virulence (Merino and Tomás, 2014).

Chemotaxis is the mechanism by which Campylobacter and other bacteria sense and move toward more favourable conditions and/or nutrients (Lertsethtakarn et al., 2011). In the chicken gut $C$. jejuni uses chemotaxis to locate the mucus filled crypts to proliferate (Chang and Miller, 2006). The primary chemoattractants are mucins and glycoproteins, which make up the mucus in the chicken gut (Hermans et al., 2012). Many chemoattractants for Campylobacter have been found, including some metabolic substrates such as: L-aspartate, L-asparagine, L-cysteine, L-glutamate, pyruvate and Lserine, as well as electron donors and electron acceptors including L-malate, succinate, fumarate, nitrite, and nitrate (Guccione et al., 2008; Mohammed et al., 2004; Velayudhan et al., 2004; Weingarten et al., 2008; Westfall et al., 1986). There are also substances which repel Campylobacter such as bile salts (Beery et al., 1988; Negretti et al., 2017). The sensing of chemoattractants and chemorepellents is mediated by methylaccepting chemotaxis proteins which possess a large cytoplasmic signalling and adaption domain and a periplasmic ligand interaction domain. There are four proteins, CheA, CheY, CheW and CheZ, which are responsible for transduction of the signal from the receptors of the flagellar motor (Lertsethtakarn et al., 2011).

\subsubsection{Adhesion}

Campylobacter adheres to host gastrointestinal epithelial cells, aiding colonisation and potentiating infection. This is mediated by several adhesins (Table 1.1) on the bacterial cell surface (Bolton, 2015; Jin et al., 2001). Campylobacter is able to adhere to fibronectin in the extracellular matrix, and this is mediated by $\mathrm{CadF}$, a $37 \mathrm{kDa}$ fibronectin-binding outer membrane protein, encoded by the $c a d F$ gene (Hofreuter et al., 2006). The binding of Campylobacter to fibronectin triggers a signalling process that leads to the activation of the GTPases Rac1 and Cdc42 which induce Campylobacter invasion and cell internalisation (Bolton, 2015).

Genes such as pebla, peb3 and peb4 may also have a function in adhesion. pebla, which encodes a $21-\mathrm{kDa}$ periplasmic binding protein has been found commonly in $C$. jejuni strains (Bolton, 2015). peb3, a transport protein is involved in the utilisation of 3 phophoglycerate (Min et al., 2009), peb4 has a main function as a chaperone protein, 
with an important role in exporting proteins such as CapF to the outer membrane (Kale, 2011).

Campylobacter adhesion protein (CadA), encoded by cadA, appears more multifunctional than its name would suggest being involved as either an autotransporter lipoprotein or as a putative protein with a role in either adhesion or invasion (Ashgar et al., 2007; Flanagan et al., 2009; Jin et al., 2001). FlpA, encoded by flpA, has a role in binding fibronectin and may act along with $\mathrm{CadF}$ during $C$. jejuni adhesion and invasion (Eucker and Konkel, 2012). Another protein with involvement in adhesion is a 42-kDa lipoprotein encoded by $j l p A$ which appears to bind to a $90-\mathrm{kDa}$ heat shock protein host cell receptor (Jin et al., 2003) .

Table 1.1. Factors and genes which have been previously shown to play a role in the adhesion of Campylobacter to the host gut epithelia

\begin{tabular}{|l|l|l|}
\hline Adhesion Factor & $\begin{array}{l}\text { Encoding } \\
\text { Gene }\end{array}$ & Reference \\
\hline CadF, Outer Membrane protein & $c a d F$ & (Konkel et al., 1997) \\
\hline CapA, Campylobacter adhesion protein A & $c a p A$ & (Jin et al., 2001) \\
\hline Phopholipase A. & pldA & (Ziprin et al., 2001) \\
\hline Peb1, periplasmic binding protein & $p e b A 1$ & (Flanagan et al., 2009) \\
\hline Peb3, transport protein & $p e b 3$ & (Min et al., 2009) \\
\hline $\begin{array}{l}\text { Peb4, chaperone involved in exporting proteins } \\
\text { to the outer membrane }\end{array}$ & $p e b 4$ & (Kale, 2011) \\
\hline Lipoprotein & jlpA & (Jin et al., 2001) \\
\hline FlpA, fibronectin-like protein A & $f l p A$ & (Flanagan et al., 2009) \\
\hline
\end{tabular}

\subsubsection{Invasion and internalisation}

There are two proposed mechanisms currently by which $C$. jejuni could invade a host epithelial cell, the 'trigger' and 'zipper' ones (Figure 1.2). The 'Zipper' mechanism begins with one or more bacterial surface proteins, commonly comprising surface adhesins and invasins such as CadF, JlpA and FlpA, which bind to specific host cell receptors followed by internalisation (Figure 1.2a). In contrast the 'Trigger' mechanism involves type-III and type-IV secretion systems (T3SSs and T4SSs) injecting bacterial 
proteins, such as cytolethal distending toxin (CDT) and CiaI, which can hijack or mimic specific host cell factors triggering the bacterial uptake process (Figure 1.2b; (O Cróinín and Backert, 2012).

Initiation of the 'Zipper' mechanism begins when bacterial pathogens adhere to the intestinal epithelium, the bacterial surface adhesins, such as $\mathrm{CadF}$, bind to their cognate receptors on cells with high affinity, which then initiates the cytoskeleton-mediated 'zippering' of the host cell's plasma membrane around the bacterium (Figure 1.2a). The bacterium is then internalised into a vacuole (Figure 1.2a) (O Cróinín and Backert, 2012). Initiation of the 'Trigger' begins with interaction with the host intestinal epithelium through fibronectin binding domains present in CadF and FlpA. Campylobacter then use sophisticated T3SSto inject various effector proteins, such as Cia (Konkel et al., 1999, 2004), into the host cells cytoplasm. These effector proteins modulate a wide range of signalling events including the activation of small Rho GTPases and cytoskeletal reorganisation to induce membrane ruffling and then bacterial uptake (Figure 2b). As a result of the signalling, the bacteria are internalised into a vacuole (Figure 2b) (O Cróinín and Backert, 2012). It is unclear which mechanism $C$. jejuni employs to invade a cell; some studies have shown that it induces membrane ruffling in a contact-dependent manner followed by host cell entry; first with its flagellar tip followed by the opposite flagellar end which seems to share features of both the 'zipper' and 'trigger' mechanisms (Boehm et al., 2011; Krause-Gruszczynska et al., 2007).

The flagella T3SS are able to deliver the gene products of flaC and cia into the cell's cytoplasm which is very important for colonisation and invasion into epithelial cells (Konkel et al., 2004). CiaC, encoded by ciaC, is required for invasion into host cells, while CiaI, encoded by cial, has been reported to be required for intracellular survival of C. jejuni after invasion (Table 1.2; (Buelow et al., 2011; Eucker and Konkel, 2012). The invasion-associated proteins are encoded by iamA, and their exact role in the process is still unclear (Rivera-Amill et al., 2001), while, HtrA, encoded by htrA, may be used as a chaperone protein which affects folding of adhesins (Bæk et al., 2011).

C. jejuni strains with mutations in glycosylation pathways have been shown to have defects in their ability to adhere to and invade host cells (Kakuda and DiRita, 2006; Szymanski et al., 2002). Invasion by $C$. jejuni has also been shown to occur due to its ability to activate MAP kinases leading to the production of pro-inflammatory cytokine 
Interleukin-8 (IL-8; (Hickey et al., 2000). Indeed, bacterial internalisation into intestinal epithelial cells is very important in $C$. jejuni pathogenesis (Watson and Galán, 2008). C. jejuni is internalised into intestinal epithelial cells in a microtubule-dependent, actinindependent fashion which is very different from many other bacterial pathogens, such as Salmonella Typhimurium and Shigella flexneri, which use the host cell actin cytoskeleton to gain intracellular access (Watson and Galán, 2008).

Table 1.2. Factors which affect invasion of $C$. jejuni into epithelial cells and their encoding genes.

\begin{tabular}{|c|c|c|}
\hline Invasion Factor & $\begin{array}{l}\text { Encoding } \\
\text { Gene }\end{array}$ & Reference \\
\hline CiaB, Campylobacter invasion antigen & $\operatorname{ciaB}$ & $\begin{array}{l}\text { (Konkel et al., } \\
2004)\end{array}$ \\
\hline CiaC, Campylobacter invasion antigen & CiaC & $\begin{array}{l}\text { (Christensen et al., } \\
\text { 2009) }\end{array}$ \\
\hline $\begin{array}{l}\text { CiaI, May be required for survival } \\
\text { intracellularly }\end{array}$ & CiaI & $\begin{array}{l}\text { (Buelow et al., } \\
2011)\end{array}$ \\
\hline $\begin{array}{l}\text { HtrA, chaperone protein involved in folding of } \\
\text { adhesins }\end{array}$ & htrA & (Bæk et al., 2011) \\
\hline IamA, Invasion associated protein & $\operatorname{iamA}$ & $\begin{array}{l}\text { (Carvalho et al., } \\
\text { 2001) }\end{array}$ \\
\hline FspA, role in apoptosis & fspA & (Poly et al., 2007) \\
\hline $\mathrm{ABC}$ transporter ATP-binding protein & $\operatorname{iamA}$ & $\begin{array}{l}\text { (Rivera-Amill et al., } \\
\text { 2001) }\end{array}$ \\
\hline
\end{tabular}


A

1. Infection

C. jejuni

Adhesins on surface

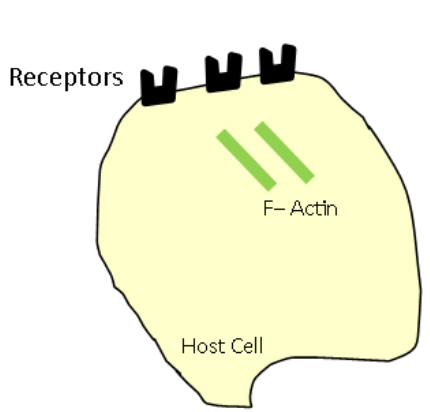

2. Receptor Binding

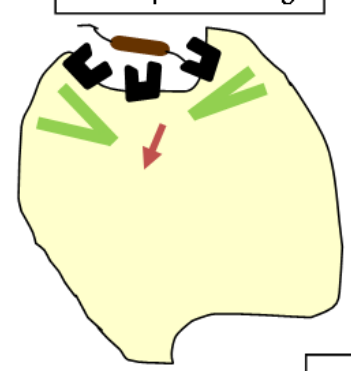

3. Membrane engulfment

4. Endosomal Trafficking

B

1. Infection
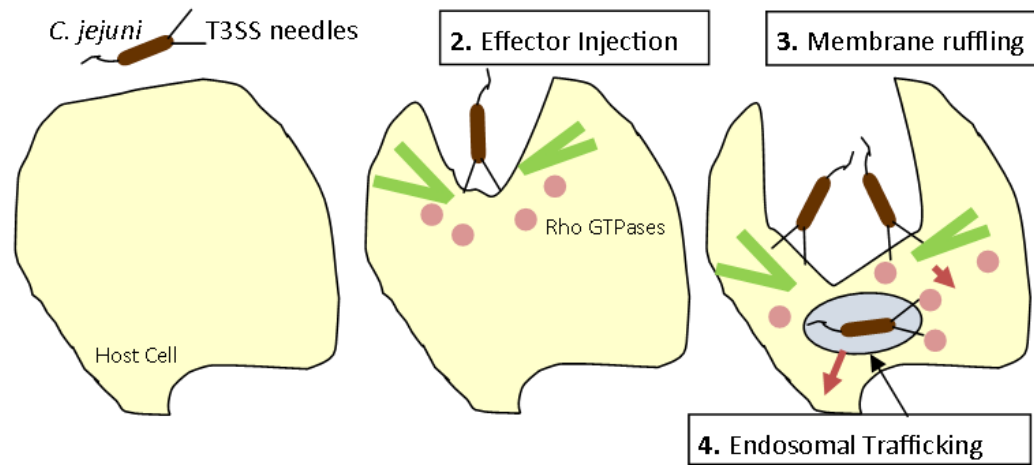

Figure 1.2. Visual representation of the 'zipper' (a) and 'trigger' (b) mechanisms $C$. jejuni uses to invade a host cell 


\subsubsection{Intracellular survival}

After internalisation into epithelial cells, C. jejuni remains within the membrane bound compartment. With time, it becomes either oxygen sensitive or may change its respiratory pathway so that it can no longer grow in the presence of oxygen. The compartments within which $C$. jejuni survives are distinct from lysosomes and are termed vacuoles. They acquire different markers transiently such as Rab7, of the endocytic pathway, and survive in a compartment that is functionally separated from the canonical endocytic one involved in host defence (Cossart and Sansonetti, 2004). C. jejuni has been shown to survive within intestinal epithelial cells by preventing fusion of lysosomes, thus manipulating the canonical endocytic pathway to generate a unique intracellular compartment conducive for survival (Watson and Galán, 2008). C. jejuni containing vacuoles are inaccessible to endocytic tracers, which could show that they are functionally separate from the normal endocytic pathways leading to lysosomes. This suggests that $C$. jejuni has evolved specific adaptations to traffic in the host cell to avoid delivery into the lysosome and therefore survive within the cell (Watson and Galán, 2008).

\subsubsection{Metabolic and environmental regulation}

Campylobacter can survive under different environmental and host stressors. For instance, iron acquisition plays an important role in the virulence of Campylobacter through the use of heme compounds such as ferric iron to sustain nutrients within the host (Bolton, 2015; Braun, 2001; Hermans et al., 2011). Iron is very important for anaerobic respiration, electron transport and energy metabolism (Braun, 2001). Another virulence factor which plays a role in Campylobacter is superoxide dismutase which helps to remove the reactive oxygen species superoxide which damages cellular DNA, proteins and membranes (Nakajima et al., 2014; Purdy et al., 1999).

Increasingly, antibiotic resistance has been observed in Campylobacter, with resistance to erythromycin, tetracycline, ciprofloxacin, kanamycin, chloramphenicol and nalidixic acid commonly occurring, which may help the survival and virulence of the bacteria within the host environment (Bolton, 2015; Silva et al., 2011). 


\subsubsection{Endotoxin and exotoxin production}

\subsubsection{Endotoxin production}

Campylobacter also contain virulence factors based on its cell wall; lipopolysaccharides (LPS) and lipooligosaccharides (LOS) in the outer membrane help to contribute to adhesion as well as in evasion of the host immune system. Campylobacter are able to change the antigen composition of LOS/LPS which makes it more difficult for the host immune system to detect the pathogen (Ang et al., 2002; Bax et al., 2011). An important pathogenicity factor in Guillian-Barré syndrome is the sialic acid that is contained in the core oligosaccharide of Campylobacter which resembles gangliosides which can cause the neurological disease by initiating specific $\mathrm{T}_{\mathrm{H}}$ differentiation programs (Ang et al., 2002; Bax et al., 2011).

\subsubsection{Exotoxin production}

Many strains of Campylobacter have the ability to produce toxins, which affect host cells in multiple ways (Wassenaar, 1997). Campylobacter has two types of toxins, entero- and cytotoxins (Albert et al., 2007; Lin et al., 2011; Thelestam and Frisan, 2004; Wassenaar, 1997). Enterotoxins such as CDT bind to cellular receptors; enter the host cell and are able to elevate intracellular cyclic adenosine monophosphate (AMP) levels. In contrast, cytotoxins are secreted proteins that kill target cells. They act intracellularly and form pores in the cells, showing a different mechanism of toxicity. They involve either inhibition of cellular protein synthesis or actin filament formation (Khan, 2005; Silva et al., 2011; Wassenaar, 1997).

One of the main toxins produced by $C$. jejuni is CDT, which causes direct DNA damage leading to the activation of checkpoint pathways and eventual cell death (Lee et al., 2003). CDT has been further classified as a genotoxin which consists of three protein subunits; CdtA, CdtB and CdtC encoded by the genes $c d t A, c d t B$ and $c d t C$. Expression of all three genes is required in order to produce an active form of CDT (Gargi et al., 2012; Lai et al., 2016; Pickett et al., 1996). CdtB is a DNase that causes DNA doublestrand breaks in the host cell nucleus resulting in cell cycle arrest at the $G_{2} / M$ stage of the cell cycle causing apoptosis (Lai et al., 2016). CdtA and CdtC bind to cholesterolrich micro-domains on the cytoplasmic membrane which are required for CdtB uptake into cells; the mechanism by which $\mathrm{CdtA}$ and $\mathrm{CdtC}$ interact with cholesterol remains unclear (Lai et al., 2016). Some Campylobacter strains have also been shown to have haemolytic properties (Wassenaar, 1997). 


\subsection{The Epithelial cell}

The surface of the gastrointestinal tract (GI) is covered by a single layer of epithelium that separates the intestinal tissue from the lumen of the intestine (Kunisawa and Kiyono, 2013). Intestinal epithelial cells (IECs), which form the barrier between the lumen of the gut and underlying tissues are short-lived and are renewed continuously, a process relying on proliferative stem cells which reside in the crypt base of the intestinal glands. The new cells are formed at the base and migrate upwards out of the crypt which eventually leads to apoptosis of the old ones and thus there is a constant number of epithelial cells in the gut (van der Flier and Clevers, 2009). Epithelial cells are responsible for apical uptake of nutrients and their transfer to the basolateral membrane for distribution to body tissues through the circulation (Klunder et al., 2017). IECs also perform another vital role in that they are the first line of defence against potential pathogens passing through the gastrointestinal tract while also maintaining relationships with commensal microbiota which play large roles in normal gut health. The defence against pathogens is achieved in part by the physical barriers of the epithelium including tight junctions (TJs) and mucus layers (Kunisawa and Kiyono, 2013). Intestinal tissues show intense immunological activity with IECs contributing to the intestinal immune system through transport and processing of antibodies and antigens, by interacting with immunocompetent cells, such as lymphocytes, in the intestine and by producing immunologically functional molecules including cytokines and antimicrobial peptides (Kunisawa and Kiyono, 2013).

IECs are arranged in a monolayer of columnar shaped polarised epithelial cells and communicate with the environment through many receptors, transporters and channels on the surface, made up of an apical and a basolateral domain. The basolateral cellsurface can be divided further into a basal and lateral which face the basement membrane and neighbouring cells, respectively (Klunder et al., 2017). Claudin-based TJs, E-cadherin-based adherens junctions (AJs) and intercellular adhesions, are located on the lateral surface which give the monolayer strength and impermeability (Giepmans and van IJzendoorn, 2009). TJs are composed of many interacting cellular proteins, such as claudin and occludin. Claudin and occuldin are transmembrane proteins that bridge the gap and seal the space between parallel IECs. There are numerous types of claudins expressed in the intestinal epithelium including, claudin $-1,-2,-3,-4,-5,-7,-8$, $-12,-15,-18,-20$ and -23 (Tamura et al., 2008; Ulluwishewa et al., 2011). Integrin- 
based cell matrix adhesions are found on the basal surface, by which the cells are attached the basement membrane (Klunder et al., 2017).

\subsection{Mucosal Immunity}

\subsubsection{The human gut}

Mucosal membranes are integral part of the gut and are major sites of entry for many pathogens (Bamias et al., 2014; Owen et al., 2013; Perez-Lopez et al., 2016). They are protected by a group of organised lymphoid tissues known as mucosa-associated lymphoid tissue (MALT) and specifically the gut-associated lymphoid tissue (GALT) in the intestinal walls (Owen et al., 2013; Zucca and Bertoni, 2016).

Lymphoid cells are found in various regions within the intestine; such as intraepithelial lymphocytes (IELs), many of which are $\mathrm{T}$ cells, specifically in the outer mucosal epithelial layer. The mucus layer overlying the epithelium secreted by goblet cells promotes elimination of gut contents and provides first line defense against physical and chemical injury caused by food and microbes (Kim and Ho, 2010). The lamina propria, which is under the epithelial layer, contains high quantities of B cells, and activated plasma cells and macrophages (Bekiaris et al., 2014; Owen et al., 2013). An example of the extensive MALT system in the intestine are Peyer's patches; made up of nodules of 30 to 40 lymphoid follicles which extend into the muscle layers that are just below the lamina propria (Yasuda et al., 2002). The functional importance of MALT in the body's defence is emphasised by its large population of antibody-producing plasma cells as well as some activities and cellular structures which are unique to it. Delivery of small samples of foreign antigens from the intestinal tracts to the underlying MALT is achieved by specialised M cells (Kanaya and Ohno, 2014). These have 'deep pockets' in the basolateral plasma membrane which contain clusters of $\mathrm{B}$ and $\mathrm{T}$ cells and macrophages which allow them to gain access to antigen. Antigens in the intestinal lumen are endocytosed into vesicles that are transported from the lumen to the underlying 'pocket' where they then fuse with the membrane, delivering the antigen to clusters of lymphocytes and dendritic cells within the pocket (Banchereau et al., 2000). Antigens which are transported by $\mathrm{M}$ cells ultimately lead to the activation of B cells, which can then differentiate and secrete $\operatorname{IgA}$, an antibody with a critical role against infection at mucosal sites (Fagarasan and Honjo, 2003). IgA has the ability to neutralise both toxins and pathogens, continually interacting with commensal bacteria on the mucosal surfaces preventing them from entering the bloodstream (Fagarasan and Honjo, 
2003). IgA can exist either in a monomeric form, usually in circulation, or form dimers and polymers in mucosal tissues. The dimeric and polymeric forms can bind to receptors, such as TLR4 and TLR5, on epithelial cells which can trigger endocytosis and transportation of molecules such as antigens from the basolateral to apical side of the epithelial cell and into the tissue lumen (Fagarasan and Honjo, 2003). In humans, two subclasses of IgA exist: $\operatorname{Ig} \mathrm{A} 1$, found in serum and $\operatorname{IgA} 2$, found more commonly in secretions (Lavelle et al., 2010; Lim et al., 2011; Mcdermott and Huffnagle, 2014; Owen et al., 2013; Perez-Lopez et al., 2016).

Paneth cells are highly specialised epithelial cells present along the small intestine in the crypts of Liberkuhn, tiny invaginations that line the mucosal surface. Paneth cells secrete antimicrobial peptides and proteins which are key mediators of host-microbe interactions such as colonisation of microbiota and innate immune protection from enteric pathogens (Clevers and Bevins, 2013).

\subsubsection{The chicken gut}

The GI of chickens is much shorter than that of mammals relative to body length, thus the digesta move through it more rapidly (Pan and $\mathrm{Yu}, 2014$ ). The short retention time selects for bacteria with 'strong' adhesion properties to the mucosal layer. However, attached to the chicken colon are two caeca, made up of two pouches, the contents of which have quite slow turnover rates, and when the animal is well provide optimal conditions for a diverse microbiome that can have a large impact on host health (Pan and $\mathrm{Yu}, 2014)$.

The GALT in chickens consist of similar structures to those seen in mammals with organised tissues including single and multiple lymphoid follicles, lamina propria lymphocytes, Peyer's patches and various lymphoid aggregates across the GI tract (Befus et al., 1980; Brisbin et al., 2008; Casteleyn et al., 2010). The Peyer's patches in chickens have similar roles to those in mammals, which includes antigen sampling, although they are not as prominent in the chicken GI tract (Vaughn et al., 2006). Chickens also contain caecal tonsils, the largest lymphoid organ in the chicken gut located in the caecum-rectum junction (Figure 1.3) (Casteleyn et al., 2010; Kitagawa et al., 1998). 
Chickens and other avianspecies have a unique Bursa of Fabricius and a Meckel's diverticulum. The bursa of Fabricius is located behind the cloaca and is the site of primary B cell development in chickens (Yasuda et al., 2002). The organ acts as both a primary lymphoid organ and a secondary one, as the mucosal and submucosal regions of the bursal canal contain multiple lymphoid follicles (Muir et al., 2000). Meckel's diverticulum is a remnant of the yolk sac present in the small intestine and its precise function is still somewhat unclear although it may be an induced lymphoid organ (Muir et al., 2000; Oláh et al., 1984). Lymphoid aggregates, which are normally found scattered throughout the epithelium and the lamina propria, possess a morphologically distinct epithelial layer called the follicle-associated epithelium (FAE). M cells are found within the FAE which have a similar function of transporting antigens from the lumen to follicular environments, as seen in mammalian cells (Brisbin et al., 2008). The lamina propria contains a variety of cell types including plasma cells, effector and memory lymphocytes, macrophages and granulocytes.

The intestinal epithelium of chickens has a similar composition to mammalian systems containing $\mathrm{M}$ cells along with intra-epithelial lymphocytes (IEL), which are distributed throughout the intestinal epithelium, goblet cells and paneth cells (Wang et al., 2016). The IEL comprise a diverse population of lymphocytes which include B cells, T cells and natural killer cells (Göbel et al., 2001). In chickens, IgA is present in monomeric, dimeric and polymeric forms in sera and intestinal and mucosal secretions. However, in serum the concentration of $\operatorname{IgA}$ is much lower than that found in secretions (Fagarasan and Honjo, 2003). The main structural differences between the human and chicken gastrointestinal tract can be seen in figure 1.3. 


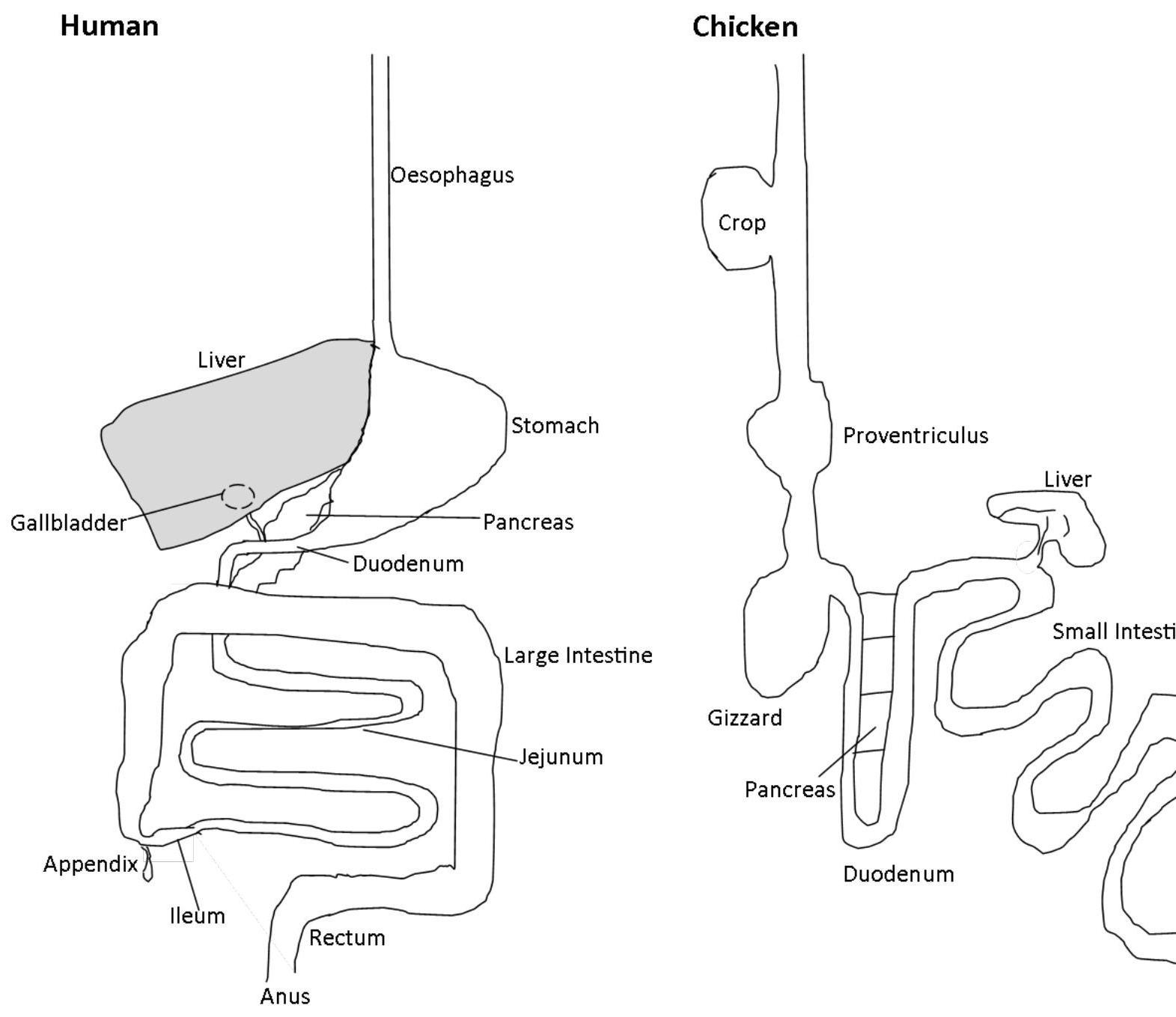

Figure 1.3. Outline of the gastrointestinal tract in human and chicken systems 


\subsection{Toll-Like Receptors and signalling}

\subsubsection{Toll-like receptors and ligands}

Toll-like receptors (TLRs) are a family of pattern recognition receptors (PRRs) which recognise and bind pathogen-associated molecular patterns (PAMPs) and damageassociated molecular patterns (DAMPs) leading to activation of immune cell responses (Takeda and Akira, 2005). Currently, 11 TLRs have been identified in humans (although TLR11 may not be functional) and 10 in chickens and these are outlined in Table 1.3 (Kannaki et al., 2010; Keestra et al., 2013; Takeda and Akira, 2005).

PAMP or DAMP binding to specific TLRs induces receptor dimerisation (Takeda and Akira, 2005). Most TLRs dimerise with themselves to form a homodimer. However, TLR2 forms a heterodimer by pairing with either TLR1 or TLR6. TLRs that recognise PAMPs on the outer surface of microbes are found on the plasma membrane. TLRs that bind to microbial ligands can also recognise DAMPs; endogenous components released by dead cells or damaged tissues. Among DAMPs recognised by plasma membrane TLRs are chromatin and heat shock proteins, fragments of extracellular matrix components and oxidised low density lipoprotein and amyloid- $\beta$ (Kawasaki and Kawai, 2014; Murphy et al., 2007). Other TLRs, such as TLR3, 7 and 9 are intracellular and recognise internal microbial components that have to be exposed by the degradation of endocytosed pathogens, such as nucleic acids, and are found in lysosomes and endosomes (Kawasaki and Kawai, 2014). 
Table 1.3. List of TLRs and ligands found in humans and chickens (Kannaki et al., 2010; Keestra et al., 2013; Owen et al., 2013; Takeda and Akira, 2005).

\begin{tabular}{|l|l|l|}
\hline Human TLR & Chicken TLR & Ligand \\
\hline TLR1 & $\begin{array}{l}\text { TLR1LA/2A, TLR1LB/2A, } \\
\text { TLR1LA,2B }\end{array}$ & Triacylatedlipopeptides \\
\hline TLR2 & TLR1LA/2A, TLR1LB/2A & Diacylatedlipopeptides \\
\hline TLR3 & TLR3 & dsRNA \\
\hline TLR4 & TLR4 & LPS \\
\hline TLR5 & TLR5 & Flagellin \\
\hline TLR6 & TLR1LA/2A, TLR1LB/2A & Diacylatedlipopeptides \\
\hline TLR7 & TLR7 & ssRNA \\
\hline TLR8 & Not Functional & ssRNA \\
\hline TLR9 & Absent & DNA \\
\hline TLR10 & Absent & Unknown \\
\hline TLR11 & Absent & profillin/ flagellin \\
\hline Absent & TLR15 & DNA \\
\hline Absent & TLR21 & Protease \\
\hline
\end{tabular}

\subsubsection{Toll-like receptor signalling}

The signalling pathways that occur downstream of TLRs share some common elements and activate expression of important genes essential for host defence. A vital shared component is the transcription factor nuclear factor kappa-light-chain-enhancer of activated B cells (NF-кB), which is needed for inducing many innate and inflammatory genes, including those producing defensins, chemokines and cytokines, such as tumour necrosis factor (TNF)- $\alpha$, IL-1 $\beta$ and IL- 6 produced by macrophages and dendritic cells. The exact signal transduction pathway activated by a TLR dimer following PAMP/DAMP binding is usually determined by the TLR and the initial protein adaptor that binds to the TLRs cytoplasmic domain, referred to as the Toll/interleukin-1 receptor (TIR) domain (Takeda and Akira, 2005). TIR domains of TLR dimers are used as binding sites for TIR domains of adaptors that activate downstream signalling pathways. The two main adaptors are myeloid differentiation factor 88 (MyD88) and TIR-domain-containing adaptor-inducing IFN- $\beta$ factor (TRIF). All TLRs except TLR3 
bind to MyD88 and TLR4 is unique in binding both MyD88 and TRIF (Takeda and Akira, 2005).

MyD88 initiates a signalling pathway that activates the NF-кB and MAPK pathways after associating with a TLR dimer following ligand binding. TLRs found on the plasma membrane such as TLR5 use MyD88 to recruit and activate several Interleukin-1 receptor-associated kinase (IRAK) protein kinases which can bind and activate TNF receptor associated factor (TRAF) such as TRAF4 or TRAF6 (TNF receptor associated factor 6) (Takeda and Akira, 2005) (Figure 1.4). TRAF6 leads to the activation of TAK1 (Transforming growth factor $\beta$-activated kinase 1) which phosphorylates the ІкB kinase (IKK) complex. NF-кB is released once IKK phosphorylates the inhibitory ІкB subunit, causing NF-кB to enter the nucleus and activate gene expression (Oeckinghaus and Ghosh, 2009). IKK is also able to activate the MAPK signalling pathways that can result in the activation of transcriptional factors to make up the activator protein 1 (AP1) dimer which is needed for differentiation, proliferation and apoptosis (Kawai and Akira, 2009; Kawasaki and Kawai, 2014).

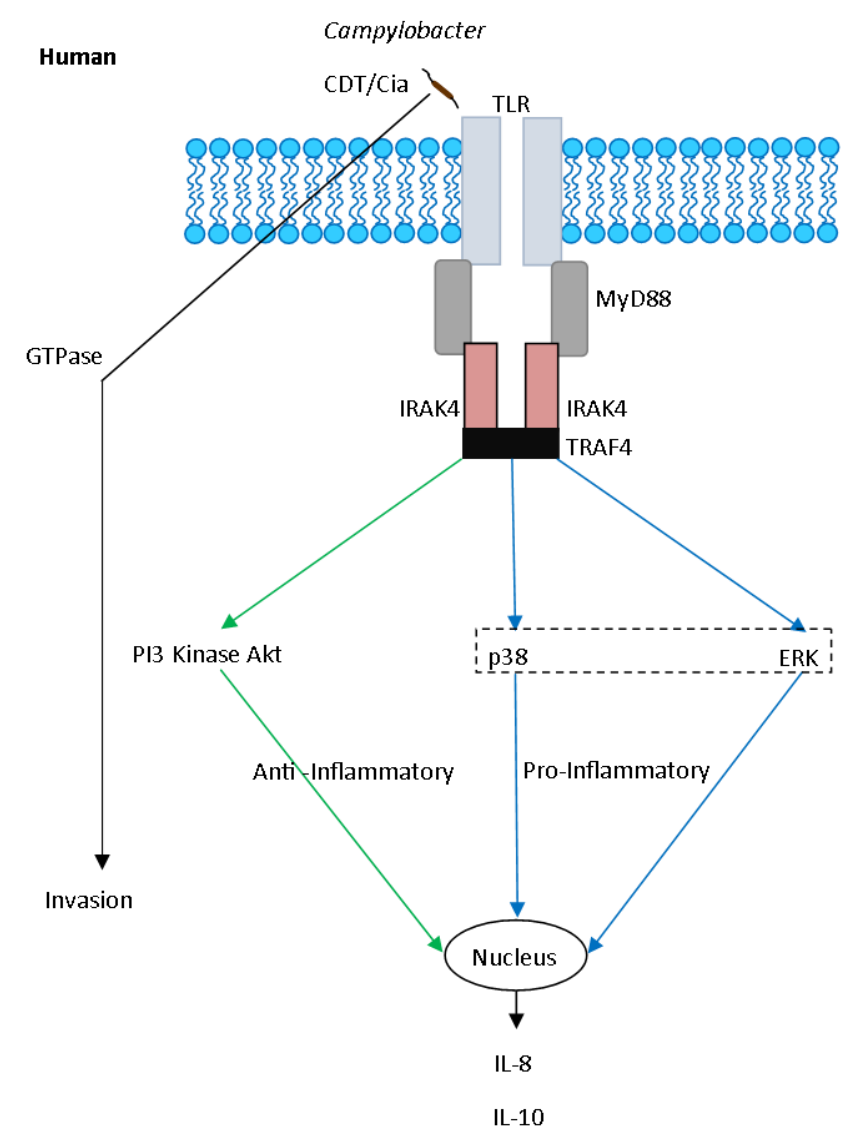

Figure 1.4. TLR Signalling caused by $C$. jejuni in the human immune systems 


\subsection{Cytokines}

\subsubsection{Structural groups based on receptors}

Cytokines are a very broad category of small proteins that are vital for intercellular signalling. There are six major cytokine families: interleukin (IL) 1, hematopoietin (class 1 cytokine), interferon (class II cytokine), TNF family, IL-17 and the chemokine family (Broughton et al., 2012; Liongue et al., 2016; Owen et al., 2013). Among the many functions of cytokines there are a large group of inflammatory ones which are produced predominately by activated macrophages and are involved in the upregulation of inflammatory reactions (Zhang and An, 2009).

\subsubsection{Functional groups based on protective immunity}

Cytokines can be classified further into the protective immunity they generate based on $\mathrm{T}$ helper subtypes; $\mathrm{T}_{\mathrm{H}} 1, \mathrm{~T}_{\mathrm{H}} 2, \mathrm{~T}_{\mathrm{H}} 17$ and $\mathrm{T}_{\mathrm{REG}}$ (Al-Banna et al., 2018). The major $\mathrm{T}$ helper cell subsets each contain polarising cytokines, which are used to induce the expression of master gene regulators that can subsequently regulate a number of effector cytokines which the T-cells can produce after they are fully differentiated (Luckheeram et al., 2012; Zhu et al., 2010). $\mathrm{T}_{\mathrm{H}} 1$ cytokines can be induced through many transcription factors such as FOXP3, T-bet and GATA3, with transcription factors T-bet, GATA3, ROR $\gamma_{t}$ inducing $T_{H} 2$ cytokines and $\mathrm{T}$-bet and $\mathrm{ROR} \gamma$ being able to induce $\mathrm{T}_{\mathrm{H}} 17$ cytokines (Evans and Jenner, 2013).

$\mathrm{T}_{\mathrm{H}} 1$ cytokines, such as IL-12, IFN- $\gamma$ and IL-18, enhance T-cell activation and protect against intracellular pathogens (Luckheeram et al., 2012; Zhu et al., 2010). $\mathrm{T}_{\mathrm{H}} 2$ cytokines, IL-4, IL-5 and IL-13, help protect against extracellular pathogens, $\mathrm{T}_{\mathrm{H}} 17$ cytokines, IL-17A, IL-17F and IL-6, contribute to inflammation and can protect against bacterial infections. In contract, $\mathrm{T}_{\mathrm{REG}}$ cytokines, IL-2, IL-10 and TGF- $\beta$ inhibit inflammation (Al-Banna et al., 2018; Luckheeram et al., 2012; Owen et al., 2013).

\subsection{3: Important pro and anti-inflammatory cytokines}

In humans and chickens, pro-inflammatory cytokines, such as IL-1, IL-6, TNF- $\alpha$ and chemokines, help to generate an immune response such as activated neutrophils (Dinarello, 2000). Anti-inflammatory cytokines are a group of immunoregulatory molecules that help control the pro-inflammatory response by acting with specific cytokine inhibitors and soluble cytokine receptors to help regulate the immune 
response. The main anti-inflammatory cytokines in both animals include IL-1RA, 4, 10, -11, -13 and IFN- $\alpha$ (Opal and DePalo, 2000; Reid et al., 2016; Su et al., 2012).

IL-8 is an important mediator in the innate immune response which attracts neutrophils and other granulocytes to a site of infection. In addition, once the neutrophils have reached such a site, IL-8 can then induce phagocytosis (Yoshimura, 2015). IL-8 plays a vital role in inflammation, as its secretion is increased by oxidant stress, causing recruitment of inflammatory cells which leads to further oxidant stress mediators (Shahzad et al., 2010). IL-8 is a potent stimulator of neutrophil activation and chemotaxis within the intestinal mucosa and has been associated with many acute and chronic inflammatory reactions (Borrmann et al., 2007). Chickens contain two orthologues for IL-8; IL-8like1 and IL-8like2 encoded by CXCLi1 and CXCLi2, respectively (Larson et al., 2008). IL-8 , in target cells, can induce migration and phagocytosis through increases in intracellular $\mathrm{Ca}^{2+}$, rapid release of reactive oxygen species (ROS) from the cell and through the release of histamine (Baggiolini, 2015; David et al., 2016a; Harada et al., 1994; Yoshimura, 2015). IL-8 is able to bind to seven transmembrane G-protein coupled receptors (GPCRs), CXCR1 and CXCR2 which mediate and regulate leukocyte accumulation and activation at sites of inflammation. CXCR1 interacts principally with IL-8. However, CXCR2 also interacts with CXCL 1, 2, 4, 5 and 7 (Raghuwanshi et al., 2012). Both monomer and homodimer versions of IL-8 are potent inducers of CXCR1 and CXCR2 (David et al., 2016a; Dinarello, 2000; Zhang and An, 2009).

IL-10, encoded by the ILIO gene in both humans and chickens, plays a major role in the anti-inflammatory response. IL-10 can down regulate expression of T-helper cell cytokines and co-stimulatory molecules on macrophages. It can also enhance B cell survival, proliferation and antibody production and is involved in the regulation of the JAK-STAT signalling pathway (Couper et al., 2008; Walter, 2014; Zdanov, 2010). IL10 is a member of the interferon family of cytokines which are secreted by T, B and dendritic cells that regulate immune responses (Owen et al., 2013; Walter, 2014). IL-10 can bind to two receptors, IL-10R1 and IL-10R2, with disparate affinities; each receptor chain has a distinct function in cellular responses when activated by IL-10 (Walter, 2014). Binding of IL-10 to IL-10R1 has been reported to control cell specificity and targeting of IL-10 to macrophages that selectively express IL-10R1 signalling. In 
contrast, IL-10R2 functions as a sensory chain, which detects IL-10 bound to IL-10R1 and therefore is used to activate signalling based on the kinetics of the IL-10/IL-10R1 interaction (Walter, 2014). Studies using IL-10-deficient mice have shown that they develop chronic inflammatory bowel disease (IBD) which may indicate that endogenous IL-10 is a central regulator of the mucosal immune system (Gomes-Santos et al., 2012; Kuhn et al., 1993; Sturlan et al., 2001).

\subsection{Aims and objectives}

The primary aim of this thesis was to understand the underlying infection biology in host pathogen interactions between populations of Campylobacter strains and the chicken. Thus, the thesis focuses on: i) using bacterial genomics to compare Campylobacter strains from defined sources including naturally infected chickens, ii) using a novel chicken intestinal epithelial cell line to study host responses, and iii) using industrial scale chicken infection studies to investigate host responses to infection. The overarching hypothesis throughout this thesis is that Campylobacter strains responsible for the invasion responses generated in vitro and in vivo can be discriminated from other strains through differences in their genomes.

More specifically aims were:

\section{Chapter 3}

- Investigate $C$. jejuni diversity and population structure in 100 strains from difference sources

- Investigate whether phenotypic differences exist between isolates in the $C$. jejuni population

- Use GWAS methods to analyse the $C$. jejuni isolates for gene associations with source.

\section{Chapter 4}

- To characterise a novel commercially available chicken epithelial cell line, 8E11

- To determine the inflammatory responses of chicken epithelial cells to $C$. jejuni strains

- To investigate how the presence/absence of known virulence genes in $C$. jejuni affect the inflammatory response 
- To investigate the signalling pathways responsible for gene expression and $C$. jejuni invasion responses

- Associate Campylobacter virulence genes to in vitro cell responses by Scroary GWAS pipeline.

\section{Chapter 5}

- Characterise the genomes of bird-matched Campylobacter from different organs in naturally infected chickens

- Identify genes responsible for colonisation/infection of ileum, caeca and liver in naturally infected chickens

- Investigate the importance of caecal tonsils from experimentally infected chickens as markers of immune responses

- Investigate in vitro cytokine and invasion responses of Campylobacter strains used to experimentally infect chickens 


\section{Chapter 2: Materials and Methods}

\subsection{Materials}

All cell culture media, antibiotics, enzymes and other media and materials used are outlined in Table 2.1.

Table 2.1. Cell Culture media, antibiotics and enzymes

\begin{tabular}{|c|c|c|c|}
\hline $\begin{array}{l}\text { Media, Antibiotics } \quad \text { and } \\
\text { Enzymes }\end{array}$ & Includes & Supplier & $\begin{array}{l}\text { Product } \\
\text { Code }\end{array}$ \\
\hline McCoy’s 5a (Invitrogen 26600) & $\begin{array}{l}\text { High Glucose } \\
\text { L-glutamine } \\
\text { Bacto-peptone } \\
\text { Phenol Red }\end{array}$ & Gibco & 16600082 \\
\hline $\begin{array}{ll}\text { DMEM/F-12, } & \text { GlutaMax }^{\text {TM }} \\
\text { Supplement } & \end{array}$ & $\begin{array}{l}\text { GlutaMAX }^{\mathrm{TM}} \\
\text { Phenol Red }\end{array}$ & Gibco & 31331028 \\
\hline Fetal Bovine Serum (FBS) & - & Gibco & 11573397 \\
\hline L-glutamine & No Phenol Red & Gibco & A2916801 \\
\hline Penicillin/ Streptomycin & - & Gibco & 15140122 \\
\hline $\begin{array}{lll}\text { Preparation } & \text { Media } & \text { (with } \\
\text { Antibiotics) } & & \end{array}$ & $\begin{array}{l}\text { 44ml McCoy's 5a or } \\
\text { DMEM/F-12 } \\
5 \mathrm{ml} \text { FBS } \\
500 \mu 1 \text { Penicillin/ Streptomycin } \\
500 \mu 1 \text { L-Glutamine }(200 \mathrm{mM})\end{array}$ & - & - \\
\hline Assay Media & $\begin{array}{l}49 \mathrm{ml} \text { McCoy's 5a or } \\
\text { DMEM/F-12 } \\
500 \mu 1 \text { FBS } \\
500 \mu 1 \text { L-Glutamine }(200 \mathrm{mM})\end{array}$ & - & - \\
\hline TrypLE express enzyme & $\begin{array}{l}\text { No Phenol Red } \\
\text { EDTA }\end{array}$ & Gibco & 12604021 \\
\hline $\begin{array}{l}\text { Phosphate Buffered Saline } \\
\text { (PBS) }\end{array}$ & - & Gibco & 10010023 \\
\hline Mueller-Hinton & $23 \mathrm{~g} / \mathrm{L}$ & Oxoid & CM0405 \\
\hline $\begin{array}{l}\text { Modified Campylobacter blood- } \\
\text { free selective medium } \\
(\mathrm{mCCDA})\end{array}$ & - & Oxoid & CM0739 \\
\hline Columbia Blood Agar (CBA) & - & Oxoid & CM0331 \\
\hline
\end{tabular}




\begin{tabular}{|c|c|c|c|}
\hline CampyGen 3.5 Litre sachet & - & Oxoid & CN0035 \\
\hline Gentamicin $(50 \mathrm{mg} / \mathrm{ml})$ & - & $\begin{array}{l}\text { Thermo } \\
\text { Scientific }\end{array}$ & 15750060 \\
\hline $\begin{array}{l}\text { Phorbol 12-myristate 13-acetate } \\
\text { (PMA) }\end{array}$ & - & $\begin{array}{l}\text { Thermo } \\
\text { Scientific }\end{array}$ & BP685-1 \\
\hline $\begin{array}{lll}\text { AlamarBlue } & \text { cell } & \text { viability } \\
\text { reagent } & & \end{array}$ & - & $\begin{array}{l}\text { Thermo } \\
\text { Scientific }\end{array}$ & DAL1025 \\
\hline iScript cDNA & $\begin{array}{l}\text { 5x iScript Reaction Mix } \\
\text { iScript Reverse Transciptase } \\
\text { Nuclease-free water }\end{array}$ & Bio-Rad & 1708890 \\
\hline SensiMix SYBR & - & Bioline & QT615-05 \\
\hline Brilliant II QPCR Mater Mix & $\begin{array}{l}\text { Brilliant II master mix } \\
\text { Reference Dye }\end{array}$ & Agilent & 600804 \\
\hline $\begin{array}{lll}\text { Human } & \text { IL-8/CXCL8 } & \text { Duoset } \\
\text { ELISA } & & \end{array}$ & $\begin{array}{l}\text { IL-8 Capture Antibody } \\
\text { IL-8 Detection Antibody } \\
\text { IL-8 Standard } \\
\text { Streptavidin conjugated to } \\
\text { horseradish-peroxidase (HRP) }\end{array}$ & $\begin{array}{l}\text { Bio- } \\
\text { Techne }\end{array}$ & DY208 \\
\hline Human IL-10 Duoset ELISA & $\begin{array}{l}\text { IL-10 Capture Antibody } \\
\text { IL-10 Detection Antibody } \\
\text { IL-10 Standard } \\
\text { Streptavidin-HRP }\end{array}$ & $\begin{array}{l}\text { Bio- } \\
\text { Techne }\end{array}$ & DY217B \\
\hline Tween 20 & - & $\begin{array}{l}\text { Thermo } \\
\text { Scientific }\end{array}$ & 003005 \\
\hline ELISA Wash Buffer & $\begin{array}{l}0.05 \% \text { Tween } \\
\text { PBS }\end{array}$ & - & - \\
\hline $\begin{array}{l}\text { SureBlue TMB } 1 \text { Component } \\
\text { Microwell Peroxidase Substrate }\end{array}$ & - & KPL & $5120-0075$ \\
\hline Hydrochloric Acid (HCI) & - & $\begin{array}{l}\text { Thermo } \\
\text { Scientific }\end{array}$ & A508-4 \\
\hline Crystal Violet $(0.1 \%$ w/v) & - & $\begin{array}{l}\text { Sigma- } \\
\text { Aldrich }\end{array}$ & $\mathrm{C} 0775$ \\
\hline Picric Acid & - & $\begin{array}{l}\text { Sigma- } \\
\text { Aldrich }\end{array}$ & $\begin{array}{l}197378- \\
500 \mathrm{G}\end{array}$ \\
\hline Formaldehyde & - & $\begin{array}{l}\text { Sigma- } \\
\text { Aldrich }\end{array}$ & F8775 \\
\hline Bouin's solution & $\begin{array}{l}\text { Picric Acid } \\
40 \% \text { Formaldehyde } \\
\text { Acetic acid }\end{array}$ & - & - \\
\hline SV Total RNA Isolation Kit & $\begin{array}{l}\text { RNA Lysis Buffer } \\
\text { RNA Dilution Buffer }\end{array}$ & Promega & Z3105 \\
\hline
\end{tabular}




\begin{tabular}{|c|c|c|c|}
\hline & $\begin{array}{l}\text { RNA Wash Solution } \\
\text { DNase Stop Solution } \\
\text { Yellow Core Buffer } \\
\mathrm{McCl}_{2} 0.09 \mathrm{M} \\
\text { DNase I } \\
\text { Beta-Macaptoethanol }\end{array}$ & & \\
\hline Dynasore $(80 \mu \mathrm{M})$ & - & $\begin{array}{l}\text { Sigma- } \\
\text { Aldrich }\end{array}$ & $\begin{array}{l}\text { D7693- } \\
5 \mathrm{MG}\end{array}$ \\
\hline Filipin & - & $\begin{array}{l}\text { Sigma- } \\
\text { Aldrich }\end{array}$ & SAE0088 \\
\hline Genistein & - & $\begin{array}{l}\text { Sigma- } \\
\text { Aldrich }\end{array}$ & $\begin{array}{l}\text { G6649- } \\
5 \mathrm{MG}\end{array}$ \\
\hline Chlorpromazine & - & $\begin{array}{l}\text { Sigma- } \\
\text { Aldrich }\end{array}$ & C8138 \\
\hline LY294002 & - & $\begin{array}{l}\text { Sigma- } \\
\text { Aldrich }\end{array}$ & L9908 \\
\hline $\begin{array}{l}\text { InSolution }{ }^{\mathrm{TM}} \text { AKT Inhibitor } \mathrm{V} \text {, } \\
\text { Triciribine }\end{array}$ & - & Merck & 124038 \\
\hline Methyl $\beta$ cyclodextrin & - & $\begin{array}{l}\text { Sigma- } \\
\text { Aldrich }\end{array}$ & C4555-1G \\
\hline Cytochalasin D & - & $\begin{array}{l}\text { Thermo } \\
\text { Scientific }\end{array}$ & PHZ1063 \\
\hline PD98059 & - & Invitrogen & tlr-pd98 \\
\hline
\end{tabular}

\subsection{In vitro Microbiology}

\subsubsection{Bacterial Isolates}

All isolates used were sub-cultured from a glycerol stock stored at $-80^{\circ} \mathrm{C}$. All Campylobacter strains used in this study are described in Table 2.2.

\subsubsection{Bacterial growth conditions}

Bacteria were grown on Columbia blood agar (CBA; Oxoid, Basingstoke, UK) or Campylobacter blood free selective medium (mCCDA;Oxoid) and incubated at $42^{\circ} \mathrm{C}$ under microaerobic conditions $\left(5 \% \mathrm{O}_{2}, 10 \% \mathrm{CO}_{2}, 85 \% \mathrm{~N}_{2}\right)$ using a CampGen $3.5 \mathrm{~L}$ sachet (Oxoid). For broth culture, a single colony was taken from a CBA plate, inoculated into Muller-Hinton ( $\mathrm{MH}$ ) broth (Oxoid) and incubated at $37^{\circ} \mathrm{C}$ for 24 hours aerobically. 
Table 2.2. List of all Campylobacter strains used throughout this thesis

\begin{tabular}{|c|c|c|c|c|}
\hline Number & Isolate & Species & \begin{tabular}{|l|} 
Clonal \\
complex
\end{tabular} & Source \\
\hline 1 & CAMP45 & C. jejuni & ST-45 & chicken \\
\hline 2 & CAMP61 & C. jejuni & ST-61 & cattle \\
\hline 3 & CampsClin11 & C. jejuni & ST-45 & human \\
\hline 4 & CampsClin45 & C. jejuni & ST-45 & human \\
\hline 5 & CampsClin262 & C. jejuni & ST-21 & human \\
\hline 6 & CampsClin583 & C. jejuni & ST-45 & human \\
\hline 7 & CampsClin266 & C. jejuni & ST-21 & human \\
\hline 8 & CampsClin883 & C. jejuni & ST-21 & human \\
\hline 9 & CampsClin1003 & C. jejuni & ST-45 & human \\
\hline 10 & chick2219 & C. jejuni & ST-45 & chicken \\
\hline 11 & chicka21 & C. jejuni & ST-21 & chicken \\
\hline 12 & cow55 & C. jejuni & - & cattle \\
\hline 13 & cow42 & C. jejuni & ST-42 & cattle \\
\hline 14 & chick2253 & C. jejuni & - & chicken \\
\hline 15 & chick594 & C. jejuni & ST-45 & chicken \\
\hline 16 & cow2673 & C. jejuni & - & cattle \\
\hline 17 & cow2674 & C. jejuni & ST-21 & cattle \\
\hline 18 & cow206 & C. jejuni & ST-206 & cattle \\
\hline 19 & cow38 & C. jejuni & ST-48 & cattle \\
\hline 20 & cow190 & C. jejuni & - & cattle \\
\hline 21 & cow334 & C. jejuni & ST-45 & cattle \\
\hline 22 & chicka45 & C. jejuni & - & chicken \\
\hline 23 & chick267 & C. jejuni & \begin{tabular}{|l|} 
ST-283 \\
\end{tabular} & chicken \\
\hline 24 & CampsClin230 & C. jejuni & ST-45 & human \\
\hline 25 & chick2048 & C. jejuni & ST-45 & chicken \\
\hline 26 & chick2197 & C. jejuni & \begin{tabular}{|l|} 
ST-354 \\
\end{tabular} & chicken \\
\hline 27 & chick607 & C. jejuni & - & chicken \\
\hline 28 & chick 2223 & C. jejuni & ST-45 & chicken \\
\hline 29 & cow3583 & C. jejuni & ST-42 & cattle \\
\hline 30 & cow618 & C. jejuni & ST-61 & cattle \\
\hline 31 & cow 237 & C. jejuni & ST-206 & cattle \\
\hline 32 & $\operatorname{cow} 270$ & C. jejuni & ST-403 & cattle \\
\hline 33 & cowb21 & C. jejuni & ST-21 & cattle \\
\hline 34 & cowb45 & C. jejuni & ST-45 & cattle \\
\hline 35 & cowc45 & C. jejuni & ST-45 & cattle \\
\hline 36 & cowd45 & C. jejuni & ST-45 & cattle \\
\hline
\end{tabular}




\begin{tabular}{|c|c|c|c|c|}
\hline 37 & cow53 & C. jejuni & ST-21 & cattle \\
\hline 38 & cow 104 & C. jejuni & ST-21 & cattle \\
\hline 39 & cow3189 & C. jejuni & - & cattle \\
\hline 40 & cow3201 & C. jejuni & ST-21 & cattle \\
\hline 41 & cow3205 & C. jejuni & \begin{tabular}{|l|} 
ST-206 \\
\end{tabular} & cattle \\
\hline 42 & cow137 & C. jejuni & ST-45 & cattle \\
\hline 43 & cow230 & C. jejuni & - & cattle \\
\hline 44 & cow583 & C. jejuni & ST-45 & cattle \\
\hline 45 & cow3207 & C. jejuni & ST-45 & cattle \\
\hline 46 & cow3214 & C. jejuni & ST-45 & cattle \\
\hline 47 & chick354 & C. jejuni & \begin{tabular}{|l|} 
ST-257 \\
\end{tabular} & chicken \\
\hline 48 & \begin{tabular}{|l|} 
chick51 \\
\end{tabular} & C. jejuni & \begin{tabular}{|l|} 
ST-443 \\
\end{tabular} & chicken \\
\hline 49 & chick1079 & C. jejuni & \begin{tabular}{|l|} 
ST-573 \\
\end{tabular} & chicken \\
\hline 50 & chick574 & C. jejuni & \begin{tabular}{|l|} 
ST-574 \\
\end{tabular} & chicken \\
\hline 51 & cowa45 & C. jejuni & ST-45 & chicken \\
\hline 52 & chick2213 & C. jejuni & ST-45 & \begin{tabular}{|l} 
chicken \\
\end{tabular} \\
\hline 53 & cow518 & C. jejuni & ST-21 & cattle \\
\hline 54 & CampsClin53 & C. jejuni & ST-21 & human \\
\hline 55 & cow58 & C. jejuni & - & cattle \\
\hline 56 & cowa21 & C. jejuni & ST-21 & cattle \\
\hline 57 & chickc21 & C. jejuni & \begin{tabular}{|l|} 
ST-21 \\
\end{tabular} & chicken \\
\hline 58 & chick 25 & C. jejuni & \begin{tabular}{|l|} 
ST-661 \\
\end{tabular} & chicken \\
\hline 59 & chick104 & C. jejuni & ST-21 & $\begin{array}{l}\text { chicken } \\
\end{array}$ \\
\hline 60 & chick353 & C. jejuni & \begin{tabular}{|l|} 
ST-353 \\
\end{tabular} & chicken \\
\hline 61 & chickb354 & C. jejuni & \begin{tabular}{|l|} 
ST-354 \\
\end{tabular} & chicken \\
\hline 62 & chick573 & C. jejuni & \begin{tabular}{|l|} 
ST-573 \\
\end{tabular} & chicken \\
\hline 63 & chick2568 & C. jejuni & \begin{tabular}{|l|} 
ST-661 \\
\end{tabular} & chicken \\
\hline 64 & chickc45 & C. jejuni & ST-45 & $\begin{array}{l}\text { chicken } \\
\end{array}$ \\
\hline 65 & chick19 & C. jejuni & ST-21 & chicken \\
\hline 66 & chick50 & C. jejuni & ST-21 & chicken \\
\hline 67 & chick53 & C. jejuni & ST-21 & $\begin{array}{l}\text { chicken } \\
\end{array}$ \\
\hline 68 & chick262 & C. jejuni & ST-21 & \begin{tabular}{|l} 
chicken \\
\end{tabular} \\
\hline 69 & chick266 & C. jejuni & ST-21 & chicken \\
\hline 70 & chick861 & C. jejuni & - & chicken \\
\hline 71 & chick1086 & C. jejuni & ST-21 & \begin{tabular}{|l} 
chicken \\
\end{tabular} \\
\hline 72 & chick1360 & C. jejuni & ST-21 & chicken \\
\hline 73 & chick11 & C. jejuni & ST-45 & chicken \\
\hline 74 & chick137 & C. jejuni & \begin{tabular}{|l|} 
ST-257 \\
\end{tabular} & chicken \\
\hline 75 & chick1003 & C. jejuni & ST-45 & chicken \\
\hline
\end{tabular}




\begin{tabular}{|c|c|c|c|c|}
\hline 76 & chick814 & C. jejuni & ST-661 & chicken \\
\hline 77 & chickb21 & C. jejuni & ST-21 & chicken \\
\hline 78 & chickb45 & C. jejuni & ST-45 & chicken \\
\hline 79 & chickd45 & C. jejuni & ST-45 & chicken \\
\hline 80 & chick883 & C. jejuni & ST-21 & chicken \\
\hline 81 & chick230 & C. jejuni & ST-45 & chicken \\
\hline 82 & chick2663 & C. jejuni & ST-45 & chicken \\
\hline 83 & CampsClin21 & C. jejuni & - & human \\
\hline 84 & OxClina21 & C. jejuni & ST-21 & human \\
\hline 85 & OxClinb21 & C. jejuni & ST-45 & human \\
\hline 86 & OxClina45 & C. jejuni & ST-45 & human \\
\hline 87 & OxClinb45 & C. jejuni & ST-21 & human \\
\hline 88 & starling177 & C. jejuni & ST-177 & starling \\
\hline 89 & starling682 & C. jejuni & ST-682 & starling \\
\hline 90 & starling 45 & C. jejuni & ST-45 & starling \\
\hline 91 & starling1020 & C. jejuni & ST-682 & starling \\
\hline 92 & goose1033 & C. jejuni & ST-1034 & goose \\
\hline 93 & goose702 & C. jejuni & - & goose \\
\hline 94 & goose137 & C. jejuni & ST-45 & goose \\
\hline 95 & goose696 & C. jejuni & ST-1332 & goose \\
\hline 96 & duck702 & C. jejuni & ST-702 & duck \\
\hline 97 & duck45 & C. jejuni & ST-45 & duck \\
\hline 98 & CAMP2381 & C. jejuni & - & $\begin{array}{l}\text { environmental } \\
\text { waters }\end{array}$ \\
\hline 99 & NCTC11168 & C. jejuni & ST-21 & human \\
\hline 100 & M1 & C. jejuni & ST-45 & human \\
\hline 101 & $\mathrm{C} 7$ & C. coli & 828 & Chicken Caeca \\
\hline 102 & $\mathrm{C} 8$ & C. coli & - & Chicken Caeca \\
\hline 103 & $\mathrm{C} 11$ & $\begin{array}{l}\text { C.coli/ } \\
\text { C. jejuni }\end{array}$ & $828 / 464$ & Chicken Caeca \\
\hline 104 & $\mathrm{C} 12$ & C. coli & 828 & Chicken Caeca \\
\hline 105 & $\mathrm{C} 14$ & $\begin{array}{l}\text { C.coli/ } \\
\text { C. jejuni }\end{array}$ & $828 / 354$ & Chicken Caeca \\
\hline 106 & $\mathrm{C} 15$ & C. coli & 828 & Chicken Caeca \\
\hline 107 & $\mathrm{C} 18$ & C. coli & 828 & Chicken Caeca \\
\hline 108 & $\mathrm{C} 20$ & - & - & Chicken Caeca \\
\hline 109 & $\mathrm{C} 23$ & C. coli & 828 & Chicken Caeca \\
\hline 110 & $\mathrm{C} 24$ & C. jejuni & 353 & Chicken Caeca \\
\hline 111 & $\mathrm{C} 25$ & C. jejuni & 353 & Chicken Caeca \\
\hline 112 & $\mathrm{C} 26$ & - & - & Chicken Caeca \\
\hline
\end{tabular}




\begin{tabular}{|c|c|c|c|c|}
\hline 113 & $\mathrm{C} 28$ & C. jejuni & 353 & Chicken Caeca \\
\hline 114 & C29 & C. jejuni & 353 & Chicken Caeca \\
\hline 115 & $\mathrm{I} 4 / \mathrm{G} 7$ & C. coli & - & Chicken Ileum \\
\hline 116 & I7/G7 & C. jejuni & 464 & Chicken Ileum \\
\hline 117 & I11/G11 & C. jejuni & 464 & Chicken Ileum \\
\hline 118 & I12/G12 & C. coli & 828 & Chicken Ileum \\
\hline 119 & I14/G14 & C. jejuni & 464 & Chicken Ileum \\
\hline 120 & I15/G15 & C. jejuni & 464 & Chicken Ileum \\
\hline 121 & I18/G18 & C. jejuni & 353 & Chicken Ileum \\
\hline 122 & I20/G20 & C. jejuni & 353 & Chicken Ileum \\
\hline 123 & I23/G23 & C. coli & 828 & Chicken Ileum \\
\hline 124 & I24/G24 & C. jejuni & 353 & Chicken Ileum \\
\hline 125 & I25/G25 & C. jejuni & 353 & Chicken Ileum \\
\hline 126 & I26/G26 & C. jejuni & 353 & Chicken Ileum \\
\hline 127 & I28/G28 & C. coli & 828 & Chicken Ileum \\
\hline 128 & I29/G29 & C. coli & 828 & Chicken Ileum \\
\hline 129 & L4 & C. coli & - & Chicken Liver \\
\hline 130 & L7 & C. jejuni & 5136 & Chicken Liver \\
\hline 131 & L11 & C. jejuni & 464 & Chicken Liver \\
\hline 132 & L12 & C. jejuni & 464 & Chicken Liver \\
\hline 133 & L14 & $\begin{array}{l}\text { C.coli/ } \\
\text { C. jejuni } \\
\end{array}$ & $828 / 257$ & Chicken Liver \\
\hline 134 & L15 & C. jejuni & 464 & Chicken Liver \\
\hline 135 & L18 & - & - & Chicken Liver \\
\hline 136 & L20 & C. coli & 828 & Chicken Liver \\
\hline 137 & L23 & C. jejuni & 464 & Chicken Liver \\
\hline 138 & $\mathrm{~L} 24$ & C. coli & 828 & Chicken Liver \\
\hline 139 & L25 & C. jejuni & 353 & Chicken Liver \\
\hline 140 & L26 & $\begin{array}{l}\text { C.coli/ } \\
\text { C. jejuni } \\
\end{array}$ & $828 / 464$ & Chicken Liver \\
\hline 141 & $\mathrm{~L} 28$ & C. coli & 828 & Chicken Liver \\
\hline 142 & L29 & C. coli & 828 & Chicken Liver \\
\hline 143 & CJ12662 & C. jejuni & - & \\
\hline 144 & CJ13126 & C. jejuni & - & \\
\hline
\end{tabular}




\subsubsection{Culture of human and chicken intestinal epithelial cells}

Human colon adenocarcinoma (HT-29) cells were maintained in McCoy's 5A (modified) Medium with L-Glutamine (5mM), Penicillin (10,000 I.U/ml) Streptomycin $(10,000 \mu \mathrm{g} / \mathrm{ml})(\mathrm{P} / \mathrm{S})$ and supplemented with $10 \%$ foetal bovine serum (FBS) (ThermoFisher-scientific, Loughborough, UK). MM-CHiC clone 8E11 chicken intestinal cells (Micromol, Germany) were maintained in Dulbecco's modified eagle medium/nutrient mixture F-12 (DMEM/F-12) (ThermoFisher-scientific, Loughborough, UK) with L-Glutamine, Penicillin Streptomycin (P/S) and 10\% FBS. Human epithelial colorectal adenocarcinoma cells (Caco-2) were maintained in Dulbecco's modified eagle medium/nutrient mixture F-12 (DMEM/F-12) (ThermoFisher-scientific, Loughborough, UK) with L-Glutamine, Penicillin Streptomycin (P/S) and 10\% FBS. All cell lines were grown routinely in a $175 \mathrm{~cm}^{2}$ flask at $37^{\circ} \mathrm{C}$ in a $5 \% \mathrm{CO}_{2}$ humidified incubator. Cell lines were grown until confluent and then were trypsinized (TrypLE Express) and counted using trypan blue. Cultures were routinely sub-cultured into new flasks when confluent.

\subsubsection{Infection studies: co-culture of epithelial cells and Campylobacter}

Campylobacter, grown overnight in $\mathrm{MH}$ broth, to a concentration of approximately $1 \times 10^{8}$ c.f.u/ml, was standardised by measuring the optical density (OD 600nm) of the bacterial suspension during experiments, ensuring that the same number of bacteria was added each time by diluting to an OD of $0.1\left(1 \times 10^{7} / \mathrm{ml}\right)$. New epithelial cell cultures from section 2.2 .3 were seeded at approximately $4 \times 10^{4}$ cells per $\mathrm{cm}^{2}$ in 24 -well plates and incubated until confluent. Then, the epithelial cell monolayers were infected with $50 \mu 1$ of bacterial suspension, containing approximately $3.5 \times 10^{5}$ c.f.u. per well. The infected monolayers were incubated for 4,6 and 24 hours at $37^{\circ} \mathrm{C}$ in a $5 \% \mathrm{CO}_{2}$ atmosphere to allow the bacteria to adhere and invade the epithelial cells. Uninfected cells and cells treated with phorbol 12-myristate 13-acetate (PMA) (10mM) were included in the experiments as negative and positive controls respectively. At the end of each time point the cell supernatants were collected into $1.5 \mathrm{ml}$ microcentrifuge tubes and placed into a centrifuge at $300 \mathrm{~g}$ for $5 \mathrm{~min}$, to remove any particulate material by centrifugation; the samples were then aliquoted and stored at $-20^{\circ} \mathrm{C}$ until ready to assay by cytokine ELISA. Total RNA from the cells was extracted and stored at $-80^{\circ} \mathrm{C}$ until ready to be converted to cDNA for qPCR described in section 2.2.6. 


\subsubsection{IL-8/IL-10 Enzyme linked immunosorbent assay (ELISA)}

The Human CXCL8/IL-8 DuoSet kit and Human IL-10 DuoSet kit (Bio-Techne, Abingdon, UK) was used to measure IL-8/IL-10 secretion from infected and uninfected human cell lines. Briefly, $50 \mu 1$ of a monoclonal antibody specific $(10 \mu \mathrm{g} / \mathrm{ml})$ for IL-8 / IL-10 was pre-coated onto a 96-well half area microplate overnight. Then, $50 \mu 1$ of standards (0-2000pg/ml IL-8 / 1L-10) and samples were pipetted into the wells for 2 hours. Wells were washed 3 times with wash buffer (0.05\% tween (Sigma-Aldrich), PBS) before $50 \mu 1$ of an enzyme-linked polyclonal antibody specific $(5 \mu \mathrm{g} / \mathrm{ml})$ for IL-8 / IL-10 was added to the wells for 2 hours. Wells were washed 3 times before a streptavidin HRP reagent was added for 20 minutes. Three further washes were completed before $50 \mu 1$ of a SureBlue ${ }^{\mathrm{TM}}$ substrate solution (Milford, UK) was added to the microplate wells to cause a colour development in proportion to the amount of IL-8 bound in the earlier step. The colour development was stopped and the intensity of the colour was measured at 450nm using the FLUOstar Omega plate reader (BMG-labtech, Aylesbury, UK). Unknowns were calculated from the standard curve.

\subsubsection{RNA Isolation}

Total RNA was isolated from infected HT-29 human or 8E11 chicken intestinal epithelial cells, from section 2.2.4, grown in a 24 well plate using a Promega SV total RNA isolation kit (Promega, Southampton, UK) according to manufacturers' instructions. Total RNA was quantified using a NanoDrop (Thermo scientific, Loughborough, UK) and integrity confirmed by agarose gel electrophoresis $(0.7 \%$ agarose gel using a $1 \mathrm{~kb}$ and $100 \mathrm{bp}$ ladders at $100 \mathrm{~V}$ for 1 hour).

\subsubsection{Quantitative PCR of RNA transcripts using SYBR green and probe based method}

One microgram of total RNA was converted to cDNA using an iScript kit (Biorad, Watford, UK). Quantitative PCR using SYBR green was used to amplify the gene of interest and the housekeeping gene. Each reaction $(25 \mu 1)$ contained $12.5 \mu 12 \mathrm{X}$ Sensimix SYBR buffer (Bioline, London, UK), $0.5 \mu$ l each primer $(25 \mu \mathrm{M}), 9.5 \mathrm{ul}$ purified water and $2 \mu \mathrm{l}$ cDNA. The qPCR conditions were as follows; $10 \mathrm{~min}$ at $95^{\circ} \mathrm{C}$, then 50 cycles with denaturing for $15 \mathrm{sec}$ at $95^{\circ} \mathrm{C}$, annealing for $15 \mathrm{sec}$ at temperatures specific to primers sets (Table 2.3) and synthesis at $72^{\circ} \mathrm{C}$. Reactions were performed in an iCycler (Biorad). Primer efficiency was measured using total RNA from epithelial cells infected with a reference $C$. jejuni isolate (NCTC11168) and a dilution series up to $1 / 10,000$. The log values of the Cycle threshold (CT) values were then taken and plotted 
graphically and the slopes used to calculate the efficiency using the equation ((1Slope $\left.) /\left(\log _{10}(2)\right)\right) * 100$. Relative transcriptional levels in distinct experiments were determined by using the $2^{-\Delta \Delta} \mathrm{Ct}$ method and $\beta$-actin as the reference housekeeping gene, where ${ }^{{ }^{\Delta}} \mathrm{Ct}=(\mathrm{Ct} \text { Infected }-\mathrm{Ct} \text { Housekeeping })_{\text {time }} \mathrm{x}-(\mathrm{Ct}$ uninfected $-\mathrm{Ct}$ Housekeeping) time 0 . Time $\mathrm{x}$ relates to gene of interest whereas time 0 relates to the untreated (Livak and Schmittgen, 2001). Primer sequences for human IL-8, IL-10 and $\beta$-actin and chicken CXCLi1/2 and $\beta$-actin were used to create primers for qPCR (Table 2.3). Target sequences were identified from the NCBI database and then the coding sequence (CDS) was used to generate forward and reverse primers using Primer3, selecting for amplicon sizes of 50-150 bases.

Quantitative PCR using the probe-based method was used to amplify the gene of interest and the housekeeping one, $28 \mathrm{~S}$ ribosomal RNA. Each reaction $(25 \mu \mathrm{l})$ contained 12.5 $\mu 1$ Brilliant II QPCR mastermix (Agilent, Stockport UK), 0.625 $\mu$ l each primer $(25 \mu \mathrm{M}), 0.25 \mu \mathrm{l}$ of each probe, $9.5 \mu \mathrm{l}$ purified water and $2 \mu \mathrm{l}$ cDNA. The qPCR conditions were as follows; $10 \mathrm{~min}$ at $95^{\circ} \mathrm{C}$, then 50 cycles with denaturing for $15 \mathrm{sec}$ at $95^{\circ} \mathrm{C}$, annealing for $15 \mathrm{sec}$ at temperatures specific to primers sets (Table 2.3) and synthesis at $72^{\circ} \mathrm{C}$. Reactions were performed with use of the AriaMx Real time PCR system (Agilent). Primer efficiency was measured using total RNA from epithelial cells infected with a reference $C$. jejuni isolate (NCTC11168) and a dilution series up to 1/10,000. The log values of the Cycle threshold (CT) values were then taken and plotted graphically and the slopes used to calculate the efficiency. Relative transcriptional levels within distinct experiments were determined using the Pfaffl method with Agilent

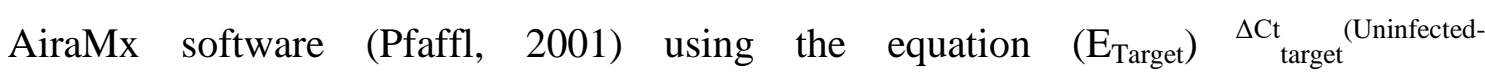
${ }^{\text {Infected })} /\left(\mathrm{E}_{\text {Housekeeping }}\right)_{\text {Housekeeping }}^{\Delta \mathrm{Ct}}{ }^{\text {(Untreated-infected) }}$, where $\mathrm{E}$ represents the efficiency of the target gene transcript.

\subsection{8 cDNA Synthesis Conditions}

Step 1: Priming at $25^{\circ} \mathrm{C}$ for $5 \mathrm{~min}$

Step 2: Reverse transcription (RT) at $46^{\circ} \mathrm{C}$ for $20 \mathrm{~min}$

Step 3: RT inactivation at $95^{\circ} \mathrm{C}$ for $1 \mathrm{~min}$

Step 4: Hold at $4^{\circ} \mathrm{C}$ 


\subsubsection{PCR Conditions}

Step 1: Inactivate at $95^{\circ} \mathrm{C}$ for $10 \mathrm{~min}$

Step 2: Denature at $95^{\circ} \mathrm{C}$ for $1 \mathrm{~min}$

Step 3: Anneal at $55-60^{\circ} \mathrm{C}$ for 1 min (Primer dependent, Table 2.3)

Step 4: Extend at $72^{\circ} \mathrm{C}$ for $1 \mathrm{~min}$

Step 5: Repeat steps 2, 3 and 4 for 50 cycles

Step 6 (SYBR only): Final extension/blunt ending at $72^{\circ} \mathrm{C}$ for $5 \mathrm{~min}$ (John et al., 2017)

Table 2.3. Primers used throughout the experiments

\begin{tabular}{|c|c|c|c|}
\hline cDNA & Primer Sequence 5'-3' & $\begin{array}{l}\text { Annealing } \\
\text { Temperature } \\
\left({ }^{\circ} \mathrm{C}\right)\end{array}$ & $\begin{array}{l}\text { Reference/ } \\
\text { Genbank }\end{array}$ \\
\hline $\begin{array}{l}\text { Chicken } \\
28 \mathrm{~S}\end{array}$ & $\begin{array}{l}\text { gcgaagccagaggaaact } \\
\text { gacgaccgatttgcacgtc }\end{array}$ & 55 & $\begin{array}{l}\text { (Shini and } \\
\text { Kaiser, 2009) }\end{array}$ \\
\hline $\begin{array}{l}\text { Chicken } \\
\text { IL- } 1 \beta\end{array}$ & $\begin{array}{l}\text { gctctacatgtgtgtgtgatgag } \\
\text { tgtcgatgtcccgcatga }\end{array}$ & 55 & $\begin{array}{l}\text { Shini and } \\
\text { Kaiser, 2009) }\end{array}$ \\
\hline $\begin{array}{l}\text { Chicken } \\
\text { IL-6 }\end{array}$ & $\begin{array}{l}\text { gctcgccggcttcga } \\
\text { ggtaggtctgaaaggcgaacag }\end{array}$ & 55 & $\begin{array}{l}\text { Shini and } \\
\text { Kaiser, 2009) }\end{array}$ \\
\hline $\begin{array}{l}\text { Chicken } \\
\text { IL-8 }\end{array}$ & $\begin{array}{l}\text { tggcaccgcagctcatt } \\
\text { tctttaccagcgtcctaccttgcgaca }\end{array}$ & 55 & $\begin{array}{l}\text { (Shini and } \\
\text { Kaiser, 2009) }\end{array}$ \\
\hline $\begin{array}{l}\text { Chicken } \\
\text { IL-10 }\end{array}$ & $\begin{array}{l}\text { catgctgctgctgggccttgaa } \\
\text { cgtctccttgatctgcttgatg }\end{array}$ & 55 & $\begin{array}{l}\text { Shini and } \\
\text { Kaiser, 2009) }\end{array}$ \\
\hline $\begin{array}{l}\text { Chicken } \\
\text { IFN } \gamma\end{array}$ & $\begin{array}{l}\text { gtgaagaaggtgaaagatatcatgga } \\
\text { gctttgcgctggattctca }\end{array}$ & 55 & $\begin{array}{l}\text { Shini and } \\
\text { Kaiser, 2009) }\end{array}$ \\
\hline $\begin{array}{l}\text { Chicken } \\
\text { TNF- } \alpha\end{array}$ & $\begin{array}{l}\text { cttctgaggcatttggaagc } \\
\text { actgggcggtcatagaacag }\end{array}$ & 55 & $\begin{array}{l}\text { (Shini and } \\
\text { Kaiser, 2009) }\end{array}$ \\
\hline $\begin{array}{l}\text { Chicken } \\
28 \mathrm{~S} \text { probe }\end{array}$ & [FAM]aggaccgctacggacctccacca[TAM] & 55 & $\begin{array}{l}\text { Shini and } \\
\text { Kaiser, 2009) }\end{array}$ \\
\hline $\begin{array}{l}\text { Chicken } \\
\text { IL- } 1 \beta \\
\text { probe }\end{array}$ & [FAM]ccacactgcagctggaggaagcc[TAM] & 55 & $\begin{array}{l}\text { Shini and } \\
\text { Kaiser, 2009) }\end{array}$ \\
\hline $\begin{array}{l}\text { Chicken } \\
\text { IL-6 } \\
\text { probe }\end{array}$ & [HEX] aggagaaatgcctgacgaagctctcca[BHQ1] & 55 & $\begin{array}{l}\text { (Shini and } \\
\text { Kaiser, 2009) }\end{array}$ \\
\hline $\begin{array}{l}\text { Chicken } \\
\text { IL-8 } \\
\text { probe }\end{array}$ & [HEX]gccetcctcctggtttgag[BHQ1] & 55 & $\begin{array}{l}\text { (Shini and } \\
\text { Kaiser, 2009) }\end{array}$ \\
\hline $\begin{array}{l}\text { Chicken } \\
\text { IL-10 } \\
\text { probe }\end{array}$ & [HEX]cgacgattcggcgctgtcacc[BHQ1] & 55 & $\begin{array}{l}\text { (Shini and } \\
\text { Kaiser, 2009) }\end{array}$ \\
\hline Chicken & [FAM]tggccaagctcccgatgaacga[TAM] & 55 & (Shini \\
\hline
\end{tabular}




\begin{tabular}{|c|c|c|c|}
\hline $\begin{array}{l}\text { IFN } \gamma \\
\text { probe }\end{array}$ & & & Kaiser, 2009) \\
\hline $\begin{array}{l}\text { Human } \\
\text { IL-8 }\end{array}$ & $\begin{array}{l}\text { cagttttgccaaggagtgct } \\
\text { ttggggtggaaaggtttgga }\end{array}$ & 57 & BC013615.1 \\
\hline $\begin{array}{l}\text { Chicken } \\
\text { IL-8-1 }\end{array}$ & $\begin{array}{l}\text { cgattgaactccgatgccag } \\
\text { cattcttgcagtgaggtccg }\end{array}$ & 59 & NM_205018.1 \\
\hline $\begin{array}{l}\text { Chicken } \\
\text { IL-8-2 }\end{array}$ & $\begin{array}{l}\text { ggatggaagagaggtgtgct } \\
\text { ctgagccttggccataagtg }\end{array}$ & 59 & NM_205498.1 \\
\hline $\begin{array}{l}\text { Human } \\
\text { IL-10 }\end{array}$ & $\begin{array}{l}\text { ggcgctgtcatcgatttctt } \\
\text { cattcttcacctgctccacg }\end{array}$ & 58 & NM_000572 \\
\hline $\begin{array}{l}\text { Chicken } \\
\text { IL-10 }\end{array}$ & $\begin{array}{l}\text { acatccaactgctcagctct } \\
\text { cgtacaggtctttgcggatg }\end{array}$ & 57 & AJ621254.1 \\
\hline $\begin{array}{l}\text { Human } \beta- \\
\text { actin }\end{array}$ & $\begin{array}{l}\text { tggcatccacgaaactacct } \\
\text { cgtacaggtctttgcggatg }\end{array}$ & 59 & X00351.1 \\
\hline $\begin{array}{l}\text { Chicken } \\
\beta \text {-actin }\end{array}$ & $\begin{array}{l}\text { aagatcattgccccacctga } \\
\text { cetgcttgetgatccacatc }\end{array}$ & 59 & L08165.1 \\
\hline $\begin{array}{l}\text { Human } \\
\text { GAPDH }\end{array}$ & $\begin{array}{l}\text { caaattccatggcaccgtca } \\
\text { atctcgctcetggaagatgg }\end{array}$ & 57 & NM_002046.5 \\
\hline $\begin{array}{l}\text { Chicken } \\
\text { GAPDH }\end{array}$ & $\begin{array}{l}\text { tgggaagctgtggagagatg } \\
\text { cttcccattcagctcaggga }\end{array}$ & 57 & NM_204305.1 \\
\hline $\begin{array}{l}\text { Chicken } \\
\text { MHC } 1\end{array}$ & $\begin{array}{l}\text { cttccacccacccaagatct } \\
\text { tcgttgaaggacatgtcgga }\end{array}$ & 58 & M84767.1 \\
\hline $\begin{array}{l}\text { Human } \\
\text { MHC } 1\end{array}$ & $\begin{array}{l}\text { ctctctcttctggcctgga } \\
\text { tctctgctggatgacgtgag }\end{array}$ & 58 & AB021288.1 \\
\hline
\end{tabular}

\subsubsection{Motility Assay}

All C. jejuni strains were grown for 48 hours on CBA at $42^{\circ} \mathrm{C}$ under microaerobic conditions $\left(5 \% \mathrm{O}_{2}, 10 \% \mathrm{CO}_{2}, 85 \% \mathrm{~N}_{2}\right)$ using CampGen 3.5L (Oxoid). A $10 \mu 1$ loop of bacteria was then re-suspended in $3 \mathrm{ml}$ of $\mathrm{MH}$ and incubated at $37^{\circ} \mathrm{C}$ overnight with shaking at 250rpm. The OD of each sample was measured ( 0.2-0.6) and it was diluted 1:10. Two millilitres of MH medium supplemented with $0.4 \%$ agar was aliquoted to each well of a 6-well plate and allowed to solidify. Then $2 \mu 1$ of $C$. jejuni suspensions $\left(0.1 \mathrm{OD}_{600}\right)$ were added to the centre of a well and the plate incubated at $37^{\circ} \mathrm{C}$ under microaerobic conditions for $48 \mathrm{~h}$. Relative motility of each bacterial strain was determined by measuring the diameter $(\mathrm{mm})$ of the migration zone with a ruler. 


\subsubsection{Invasion Assay and Adhesion}

Bacterial strains (from glycerol stocks) were inoculated onto agar plates and grown in a microaerobic environment for 48 hours at $42^{\circ} \mathrm{C}$. A colony from a freshly grown culture was sub-cultured in $\mathrm{MH}$ broth for $24 \mathrm{~h}$, as described previously in section 2.2.2. Then, $1 \times 10^{7} \mathrm{CFU}$ of bacterial suspension was added to the wells containing monolayers of cells in assay medium (modified McCoy's 5A/DMEM/F-12 with L-glutamine (5mM) and supplemented with 5\% FBS) for $6 \mathrm{~h}$. The remaining broth was serially diluted in PBS and plated out onto CBA plates for enumeration of Campylobacter. Monolayers of cells were grown in a 24 -well tissue culture plate as previously stated (section 2.2.3). For the adhesion assay, the cell monolayers incubated with bacteria were washed three times with PBS and then incubated at $37^{\circ} \mathrm{C}$ with maximum recovery diluent for $10 \mathrm{~min}$ (1.5g peptone, $8.5 \mathrm{~g}$ sodium chloride, per litre; final $\mathrm{pH} 7.0$ ) to remove unbound bacteria. Plates were shaken and adhering Campylobacter cells were removed and serially diluted in maximum recovery diluent and plated out onto CBA for enumeration. For the invasion assay, the cell monolayer incubated with bacteria were washed twice with PBS before 100 $\mu$ l gentamicin (Sigma, Poole, Dorset UK) in assay media $(100 \mu \mathrm{g} / \mathrm{ml})$ was added to each well and incubated at $37^{\circ} \mathrm{C}$ in $5 \% \mathrm{CO}_{2}$ for $90 \mathrm{~min}$. Cells infected with Campylobacter were analysed to investigate the appropriate incubation time with gentamicin and to confirm it did not affect cell integrity (Appendix Figure 8.3). Cells were washed twice with PBS before $2 \mathrm{ml} 0.1 \%$ Triton $\mathrm{X}-100$ in PBS was added to each well in order to lyse the cells. After 5 minutes, cell lysates were serially diluted in PBS and plated out on CBA plates for enumeration of the invasive bacteria. This experiment was performed four times. The limit of detection was $50 \mathrm{CFU} / \mathrm{ml}$.

\subsubsection{Cell Viability}

Cell viability was assessed using the AlamarBlue Reagent (Thermo Fisher Scientific) according to manufacturer's instructions. Briefly, $5 \mu 1$ of AlamarBlue reagent was added to each well of a 96-well plate containing HT-29 or 8E11 cells infected with $C$. jejuni in $50 \mu \mathrm{l}$ of medium. Plates were incubated for $4 \mathrm{~h}$ at $37^{\circ} \mathrm{C}$ and absorbance was measured at $\mathrm{OD}_{570}$, and $\mathrm{OD}_{600}$. AlamarBlue cell viability reagent functions as a cell health indicator using the reducing power of living cells to quantitatively measure the proliferation of cell lines. Viable cells can convert resazurin continuously, the active ingredient in alamarblue, to resorufin and so increasing the overall absorbance at $\mathrm{OD}_{570}$ and $\mathrm{OD}_{600}$ and colour of the media. 


\subsubsection{Growth and Biofilm assay.}

Growth and biofilm formation were measured using a semi-quantitative assay on Nunc 96-well tissue culture plates (Mack et al., 1992; Pascoe et al., 2015). Briefly, C. jejuni strains were grown overnight in $\mathrm{MH}$ at $42^{\circ} \mathrm{C}$ under microaerobic conditions as described in section 2.2.1, and diluted using $\mathrm{MH}$ to $0.1 \mathrm{OD}_{600}$. Five microlitres of bacterial suspension from an overnight culture (corrected to $\mathrm{OD}_{600}=0.1$ ) grown in $\mathrm{MH}$ was used to inoculate $200 \mu \mathrm{l}$ of fresh $\mathrm{MH}$-medium in a 96-well tissue culture plate. Cultures were grown and monitored in real-time over 48 hours at $37^{\circ} \mathrm{C}$ or $42^{\circ} \mathrm{C}$ in a BMG Omega plate reader using an atmospheric control unit to generate a microaerobic atmosphere. Spectrophotometric measurements were taken at $\mathrm{OD}_{600}$ every 60 mins and the average of at least three replicates was calculated. For the measurement of biofilm formation, culture medium was removed and the wells washed gently three times with PBS. Adhered bacteria were fixed with $150 \mu 1$ of bouin's solution $(7.5 \mathrm{ml}$ picric acid; $2.5 \mathrm{ml} 40 \%$ formaldehyde; $0.5 \mathrm{ml}$ acetic acid) for $15 \mathrm{~min}$ and washed again with PBS. Plates were air-dried and then stained with $0.1 \%(\mathrm{w} / \mathrm{v})$ crystal violet for $5 \mathrm{~min}$. Excess stain was removed, adhered bacteria were air dried and spectrophotometric measurements were taken at $\mathrm{OD}_{600}$ in a $\mathrm{BMG}$ Omega 96 -well plate reader and the average of at least three replicates was calculated.

\subsubsection{Inhibition Assays}

A series of known endocytosis and signalling inhibitors was used to block cellular processes (Table 2.1). HT-29 and 8E11 cells were cultured as described earlier in section 2.2.2. Cells were treated with each inhibitor separately for 30min and bacterial strains were added with subsequent invasion assays and RNA isolation/qPCR carried out as previously described in section 2.2.6.

\subsection{Bioinformatics, genomics and Campylobacter genomes}

\subsubsection{Campylobacter-BIGSdb}

The Campylobacter bacterial isolate genome sequence database (BIGsdb), (Sheppard et al., 2012) containing data from over 3000 isolates was used to identify and select strains from a variety of different sequence types and niches (human, cattle, chicken and wild bird). BIGSdb is a scalable web-database that stores contiguous sequences or whole genome data, and the corresponding origin and phenotype data for the isolates from which the sequence was derived (Jolley and Maiden, 2010; Maiden et al., 2013). 
Campylobacter BIGSdb was used to identify and characterise Campylobacter population structure for host pathogen studies in vitro and to produce gene alignments of any loci that vary in all the different strains used in this study to produce phylogenetic trees using MEGA 6 (Tamura et al., 2013).

\subsubsection{Generation of Pan-Genomes}

Genome sequences were annotated with Prokka (Seemann, 2014) and the output then used to create the pan-genome using 'Roary the pan genome pipeline' (Page et al., 2015). The resulting pan-genome was used to produce maximum likelihood phylogenetic trees using MEGA 6, iTol and phandango (Hadfield et al., 2018; Letunic and Bork, 2016; Tamura et al., 2013).

Prokka was run using the following command:

Prokka --kingdom Bacteria --outdir prokka_11168 -genus Campylobacter -locustag 11168 11168.fas

Roary was run using the following command:

roary -f ./PanGenome -e -n -v ./gff/*.gff

\subsubsection{GWAS using Scoary}

Scoary microbial pan-GWAS (Brynildsrud et al., 2016) was used to compare and analyse gene association using the 'gene_presence_absence.csv' file output from Roary along with a new 'traits' file describing the required analysis.

Scoary was run using the following command:

scoary $-\mathrm{g}$ gene_presence_absence.csv $-\mathrm{t}$ traits.csv 


\subsubsection{SNP analysis and Recombination}

Sequence outputs from Roary were taken and the gaps in them removed using Geneious (Kearse et al., 2012b, 2012a), where the sequences were then used in Gubbins (Croucher et al., 2015) to determine recombination events within the sequences as well as single nucleotide polymorphisms (SNPs).

Gubbins was run using the following command:

run_gubbins.py [FASTA alignment]

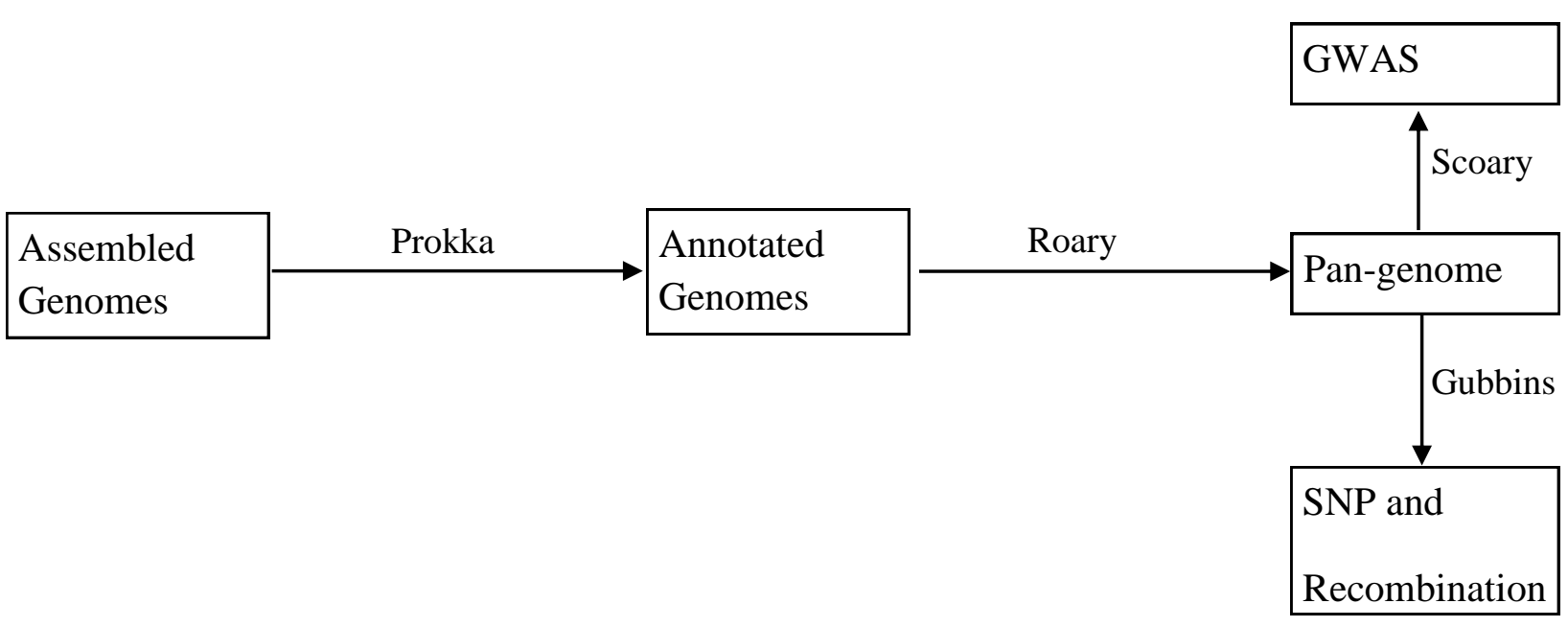

Figure 2.1. Genomic pipeline using Prokka, Roary, Scroary and Gubbins to analyse sequences 


\subsection{Programmes}

Several programmes for genomic and statistical anaylsis were used in this study, these are outlined in Table 2.4.

Table 2.4. Programs used during this study

\begin{tabular}{|c|c|c|}
\hline Programs & Version & Manufacturer \\
\hline Prokka & 1.12 & $\begin{array}{l}\text { Open Source } \\
\text { http://www.vicbioinformatics.com/software.prokka.shtml }\end{array}$ \\
\hline Roary & 3.6 .1 & $\begin{array}{l}\text { Open Source } \\
\text { https://sanger-pathogens.github.io/Roary/ }\end{array}$ \\
\hline Gubbins & 2.0 .0 & $\begin{array}{l}\text { Open Source } \\
\text { https://www.sanger.ac.uk/science/tools/gubbins }\end{array}$ \\
\hline Geneious & 10.2 .3 & Biomatters (Auckland, New Zeland) \\
\hline BioPerl & 1.007 & $\begin{array}{l}\text { Open Source } \\
\text { https://bioperl.org/ }\end{array}$ \\
\hline BLAST+ & 2.6 .0 & $\begin{array}{l}\text { Open Source } \\
\text { ftp://ftp.ncbi.nlm.nih.gov/blast/executables/blast+/LATEST/ }\end{array}$ \\
\hline HMMER & $3.1 \mathrm{~b} 2$ & $\begin{array}{l}\text { Open Source } \\
\text { http://hmmer.org/ }\end{array}$ \\
\hline Aragorn & 1.2 .36 & $\begin{array}{l}\text { Open Source } \\
\text { http://mbio-serv2.mbioekol.lu.se/ARAGORN/ }\end{array}$ \\
\hline Prodigal & 2.6 .2 & $\begin{array}{l}\text { Open Source } \\
\text { https://github.com/hyattpd/Prodigal }\end{array}$ \\
\hline tbl2asn & 23 & Open Source \\
\hline $\begin{array}{l}\text { GNU } \\
\text { Parallel }\end{array}$ & 20170922 & $\begin{array}{l}\text { Open Source } \\
\text { https://www.gnu.org/software/parallel/ }\end{array}$ \\
\hline Infernal & 1.1 & $\begin{array}{l}\text { Open Source } \\
\text { http://eddylab.org/infernal/ }\end{array}$ \\
\hline MEGA & 6 & $\begin{array}{l}\text { Open source } \\
\text { https://www.megasoftware.net/ }\end{array}$ \\
\hline iTOL & 4.2 .3 & $\begin{array}{l}\text { Biobyte solutions } \\
\text { https://itol.embl.de/ }\end{array}$ \\
\hline Phandango & 1.1 .0 & $\begin{array}{l}\text { Open Source } \\
\text { https://jameshadfield.github.io/phandango/\#/ }\end{array}$ \\
\hline Prism & 6 & GraphPad Software Inc (San Diego, USA) \\
\hline
\end{tabular}




\subsection{In vivo Experiments}

\subsubsection{Industrial scale Campylobacter infection study of broiler Chickens}

\subsubsection{1: Ethics}

An in vivo Campylobacter infection study as part of a BBSRC funded project entitled 'Broiler gut health and $C$. jejuni infection: impacts of harvest management' (BB/M009610/1) was carried out at Scotland's Rural University College (SRUC). All work was conducted in accordance with the UK legislation governing experimental animals under Home Office project licence and approved by the SRUC ethical review process.

\subsubsection{2: Campylobacter infection protocol}

Birds were infected with a cocktail of six Campylobacter strains (Appendix Table 8.2) using a litter seeding method (Sandilands et al., 2018) at a concentration of $1 \times 10^{5} \mathrm{cfu}$ of each Campylobacter. The birds were infected by members of SRUC and Drs Williams and Wilkinson of Swansea University

\subsubsection{3: Harvest of tissues}

For results generated in this thesis, the caecal tonsils were removed at the time of cull from Campylobacter infected and uninfected chickens. They were placed immediately into RNAlater (Ambicon, UK) and stored at $-20^{\circ} \mathrm{C}$ until needed.

\subsubsection{RNA isolation from caecal tonsils}

Approximately thirty $\mu \mathrm{g}$ of caecal tonsil was placed in $600 \mu \mathrm{l}$ of RNA lysis buffer with one 5mm stainless steel bead (Qiagen, Crawley UK) and was disrupted using the FastPrep FP120 (Thermo scientific, Loughborough, UK) at $4 \mathrm{~m} / \mathrm{sec}$ for 1 minute. RNA was extracted using the QIAGEN RNeasy mini kit according to manufacturer's instructions, eluted in $50 \mu 1$ nuclease free water and stored at $-20^{\circ} \mathrm{C}$ until needed. The total RNA was quantified using a NanoDrop (Thermo scientific, Loughborough, UK). 


\subsection{Statistics}

Normality was assessed using D'agostino-Pearson test. For data that were distributed normally a two-way ANOVA was used. For data that were not distributed normally, non-parametric tests were used. To compare two groups a Mann-Whitney U test was used and for 3 or more groups a Krustal-Wallis test with a post hoc Dunns test was applied.

Correlations were assessed using linear regression of $\log$ transformed data with a $\mathrm{p}$ value related to the slope. Significance differences were accepted if $p \leq 0.05$ in all tests. Graphpad Prism 6.0 (San Diego, USA) was used to analyse and assess differences between treatment groups. 
Chapter 3: Characterisation of Campylobacter jejuni strains from four different hosts

\subsection{Introduction}

\subsubsection{C. jejuni Genomics}

$C$. jejuni are distributed widely and found in the microbiota of many wild and domesticated birds and mammals and are genetically highly diverse (Killiny and Almeida, 2011). Campylobacter populations are highly structured into clusters of related lineages which include deep branching clades and clonal complexes of isolates that share four or more alleles at seven MLST loci (Dingle et al., 2001; Sheppard et al., 2008). MLST data have shown that $C$. jejuni populations are subject to high levels of horizontal gene transfer within and between species and display partial clonality where strains with similar genotypes form clusters, known as clonal complexes, which contain related STs (Sheppard et al., 2008; Smith et al., 1993). Analysis of C. jejuni populations has shown that certain clusters may be differentiated by their host sources (Colles et al., 2008; McCarthy et al., 2007; Sheppard et al., 2010b, 2010a). Studies of large collections from diverse sources and locations have identified certain STs associated with defined hosts; wild birds are often associated with their own $C$. jejuni strains (Griekspoor et al., 2013; Sheppard et al., 2011) and certain sequence types (such as ST403) are not found in birds (Morley et al., 2015).

C. jejuni is a rapidly evolving species which is subject to an intense purifying selection that can purge most novel variation while still possessing a large evolutionary potential. Recombination has a vital role in generating diversity and facilitating gene flow between $C$. jejuni (Wilson et al., 2009). In addition, the genetic basis for antimicrobial drug resistance is spread through recombination in C. jeuni (De Boer et al., 2002).

Characterisation of thousands of $C$. jejuni isolates from PubMLST (http://pubmlst.org/Campylobacter/) identified clonal complexes and STs that usually only come from the gut of a single host species and can be termed as 'host specialists'; which include chicken specialists such as STs-661,573, -574, -607, -443, -460, -283 and -257 and cattle specialists such as ST-61 and ST-42. STs-45 and -21 are found in multiple host species (Dearlove et al., 2016; Pascoe et al., 2015; Sheppard et al., 2011). 
Analysis of the genome of $C$. jejuni has identified many virulence genes which are shown in Table 3.1. Among these are ones linked to motility, toxicity, adhesion, invasion, antigen peptides, transporters and signal transduction kinases (Ashgar et al., 2007; Bæk et al., 2011; Brøndsted et al., 2005; Faber et al., 2015; Fernando et al., 2007; Joslin and Hendrixson, 2008, 2009; Kale, 2011; Khan, 2005; Koolman et al., 2016; Lee et al., 2003; Min et al., 2009; Neal-McKinney and Konkel, 2012; Pei and Blaser, 1993; Poly et al., 2007).

Table 3.1. Known virulence genes in $C$. jejuni

\begin{tabular}{|l|l|}
\hline Gene & Name/Function \\
\hline flaA & Flagellin A \\
\hline flaB & Flagellin B \\
\hline flaC & Flagellin C \\
\hline cdtA & Cytolethal distending toxin A \\
\hline cdtB & Cytolethal distending toxin B \\
\hline cdtC & Cytolethal distending toxin C \\
\hline pebla & Bi-functional adhesion/ABC transporter aspartate/ glutamate-binding protein \\
\hline peb3 & Major antigenic peptide PEB3 \\
\hline peb4 & Peptidyl-prolyl cis-trans isomerase \\
\hline pldA & phospholipase A \\
\hline jlpA & Surface exposed lipoprotein \\
\hline ciaB & Campylobacter invasion antigen B \\
\hline ciaC & Campylobacter invasion antigen C \\
\hline ciaI & Campylobacter invasion antigen I \\
\hline parA $F$ & Outer membrane fibronectin-binding protein \\
\hline
\end{tabular}




\begin{tabular}{|l|l|}
\hline htrA & Serine Protease \\
\hline$f l g R$ & Sigma-54 associated transcriptional activator \\
\hline$f l g S$ & Signal transduction histidine kinase \\
\hline$f s p A$ & flagellum-secreted non flagellar protein \\
\hline iamA & ABC transporter ATP-binding protein \\
\hline htrA & Serine protease \\
\hline
\end{tabular}

\subsubsection{Pan-Genomes}

Microbial pan-genomes were first studied in 2005 and this work showed that bacteria can contain more genes than a single genome (Medini et al., 2005). The pan-genome approach to analysing bacterial genomes gives a much wider view of a bacterial population and defines the entire genomic repertoire of a species which is made up of the core genome, genes shared in all isolates, and the accessory genome, genes shared by a subset of isolates, which contribute to species diversity and to niche lineages, which have adaptations for a new host or antibiotic resistance (Vernikos et al., 2015). Increasingly, there are more software options available in which to analyse sequences obtained from bacterial isolates. This has improved knowledge and made results more reliable thus shedding more light and obtaining a clearer picture of the relationship between sequences within a bacterial species.

\subsubsection{Recombination}

Recombination in relation to genetics is the rearrangement of DNA and has a strong impact on genetic diversity (Bishop and Schiestl, 2002; Helleday, 2010; Posada et al., 2002). The relative recombination rate of a gene shows the frequency by which a new mutation in a single gene can occur (Fearnhead et al., 2015). Recombination in bacteria is influenced by physical proximity and may lead to adaption to selective pressures, production of a new species and the exchange of DNA between distantly related bacterial strains (Chaguza et al., 2015; Fearnhead et al., 2015; Méric et al., 2015; Sheppard et al., 2008). 


\subsubsection{C. jejuni Survival}

There are many stresses that $C$. jejuni must cope with to survive in both the environment and host. These include oxidative stress, osmotic pressure, starvation, nitrosative stress, low pH and heat shock (Bronowski et al., 2014). However, C. jejuni has the ability to survive in a wide range of environments through many mechanisms such as the ability to form biofilms, undergo morphological changes and during periods of unfavourable conditions, such as a low nutrient environment, entering a purportedly viable but nonculturable (VBNC) state (Bronowski et al., 2014; Magajna and Schraft, 2015; Murphy et al., 2006; Teh et al., 2014).

Campylobacter needs to defend itself against atmospheric levels of oxygen and reactive oxygen species (ROS). Exposure of $C$. jejuni to light does not induce superoxide dismutase (SOD), the major defence against oxidative stress, instead catalase, encoded by katA, is induced but basal activity of SOD still occurs in $C$. jejuni and may play a role in defence (Bronowski et al., 2014). For optimum growth, $C$. jejuni require microaerobic growth conditions with $85 \% \mathrm{~N}_{2}, 10 \% \mathrm{CO}_{2}$ and $5 \% \mathrm{O}_{2}$ (Bolton, 2015; Davis and DiRita, 2008).

\subsubsection{Biofilm Formation}

The formation of multicellular biofilms is an ancient adaption shared by many bacteria and archaea (Pascoe et al., 2015). A biofilm is an aggregate of multiple bacterial layers encased in a polymeric substance. Biofilm is produced by $C$. jejuni and helps attachment to the surface of a biomaterial, aggregation of bacterial cells to each other and helps the survival of bacteria under stressful conditions (Joshua et al., 2006; Pascoe et al., 2015; Tolker-Neilsen, 2015). C. jejuni requires microaerobic conditions to grow and are thought not multiply in aerobic environment. However, the bacteria are found in such environments like natural waters but this may just be survival. It has been suggested that $C$. jejuni can survive in aerobic environments by forming biofilms (Joshua et al., 2006). Prevention of biofilm formation and degradation in $C$. jejuni has been observed through the use of DNase enzymes (Brown et al., 2015). In human infections, bacteria in biofilms are generally more resistant to antibiotics compared to planktonic bacteria (Joshua et al., 2006). 


\subsubsection{Aims}

In this chapter an established collection of $C$. jejuni strains (Sheppard et al., 2008, 2013) from a variety of niches and STs were used to study population structure. This study used novel genomic tools, published in the last 3 years (Brynildsrud et al., 2016; Croucher et al., 2015; Page et al., 2015), to create a pan-genome of strains along with phenotypic experiments to analyse their motility, growth and biofilm assays, to give an overall view of population behaviour. Specifically the aims were to:

- Investigate $C$. jejuni diversity and population structure in 100 strains from difference sources using novel genomic tools to create a pan-genome of strains which can then be used to analysis recombination and gene presence/absence analysis

- Investigate whether certain phenotypic differences in survival mechanisms exist between isolates in the $C$. jejuni population

- Use GWAS methods to analyse the $C$. jejuni isolates from different sources for gene associations. 


\subsection{Materials and Methods}

\subsubsection{C. jejuni isolates}

A collection of 100 fully sequenced isolates (Table 2.2 1-100) of C. jejuni from a variety of sources including 16 human, 42 chicken, 31 cattle, 10 wild birds and 1 environmental strain was used in this study. These had been characterised previously to the genomic level (Sheppard et al., 2008, 2013).

\subsubsection{Campylobacter-BIGsdb}

Campylobacter-BIGsdb (Sheppard et al., 2012) was used as described in section 2.3.1.

\subsubsection{Pan-Genome creation}

The pan-genome was created using Prokka (Seemann, 2014) and Roary (Page et al., 2015) as described in section 2.3.

\subsubsection{GWAS using Scoary}

Scoary microbial pan-GWAS (Brynildsrud et al., 2016) was performed as described in section 2.3.3. Scoary was used to identify genes that were related to the different sources of the C. jejuni isolates (human, chicken, cattle and wild bird).

\subsubsection{SNP analysis and Recombination}

Analysis of SNP and recombination using Geneious (Kearse et al., 2012b, 2012a) and Gubbins (Croucher et al., 2015) was performed as described in section 2.3.4.

\subsubsection{Growth and Biofilm assay}

The growth and biofilm assay was performed as described in section 2.2.15.

\subsubsection{Motility Assay}

The motility assay was performed as described in section 2.2.

\subsubsection{Statistics}

The two-way ANOVA with multiple comparisons and a Krustal-Wallis test with a post hoc Dunn's test for multiple comparisons were used with significance differences accepted if $p \leq 0.05$. Graphpad Prism 6.0 (San Diego, USA) was used to analyse and assess differences between treatment groups. 


\subsection{Results}

\subsubsection{MLST Analysis of $C$. jejuni populations}

We selected $C$. jejuni strains that have been described previously (Sheppard et al., 2008, 2013) . Isolates were chosen based on the availbility of the 'genome' and the 'isolate'. The MLST neighbour-joining tree was constructed with the 100 isolates (Table 2.2) used throughout this study. These were chosen from the laboratory's archived collection based on clonal complex and the source of C. jejuni (Figure 3.1).

The MLST tree (Figure 3.1) revealed three areas with two distinctive clusters which match the two main sequence types, ST-45 and -21. Both STs contain isolates from a variety of sources. In addition, a third area between these two clusters may be identified, where lineages exist for isolates from unique sources. Many of the wild bird isolates had their own unique STs, including 1034, 1332 and 702. However, some of them showed close lineage with isolates obtained from chickens such as STs-702 and -1332. 


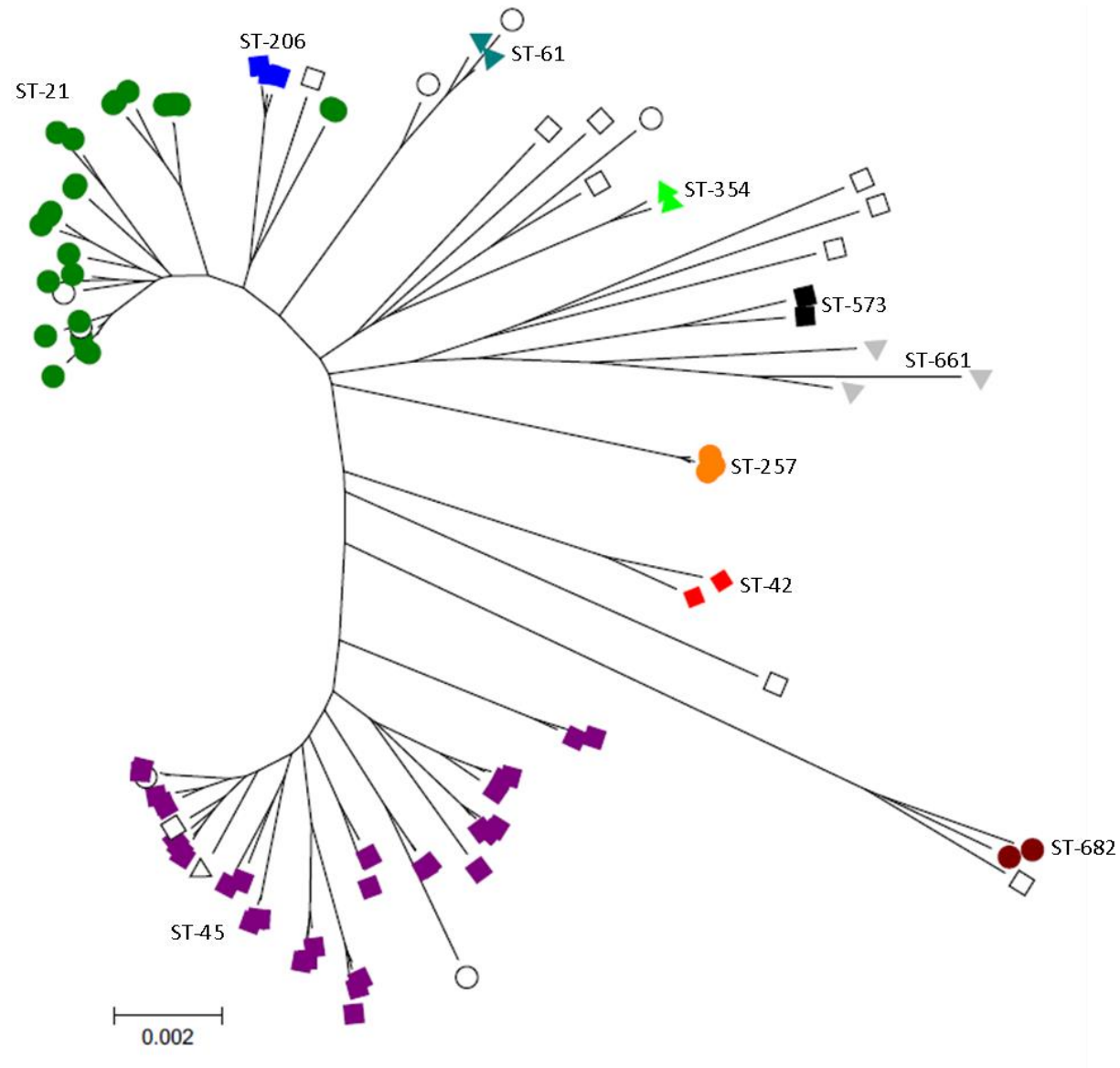

Figure 3.1. MLST tree of $100 C$. jejuni strains labelled by ST (colour), with clear points denoting strains from unqiue STs, and source (shape). Human, chicken, cattle and wild bird isolates are represented by a square, circle, triangle and diamond, respectively 


\subsubsection{Pan-genome Approach}

A pan-genome approach applying Prokka and Roary was used to construct a pangenome matrix (Figures 3.2 and 3.3). It contained 2715 genes, made up of 435 core genes $(99 \%<=$ gene presence $<=100 \%)$, with an additional 700 in the soft core $(95 \%$ $<=$ gene presence $<99 \%$ ) totalling 1135 present in the core. There were 1580 genes in the accessory genome made up of 547 shell genes $(15 \%<=$ gene presence $<95 \%)$ and 1033 cloud genes $(0 \%<=$ gene presence $<15 \%)$ (Figure 3.3). The maximum likelihood phylogenetic tree generated using Roary and MEGA6 (Figure 3.2) displayed two distinct clusters (32 strains within CC-45 and 28 strains within CC-21) both containing a mix of isolates from human and chicken sources (5 human and 18 chicken strains within CC-45 and 7 human and 14 chicken strains within CC-25). NCTC 11168 clustered with the CC-21 isolates, whereas M1, another strain used as a reference isolate, clustered with the $\mathrm{CC}-45$ isolates as expected and reported previously in Figure 3.1 (Figure 3.2). 


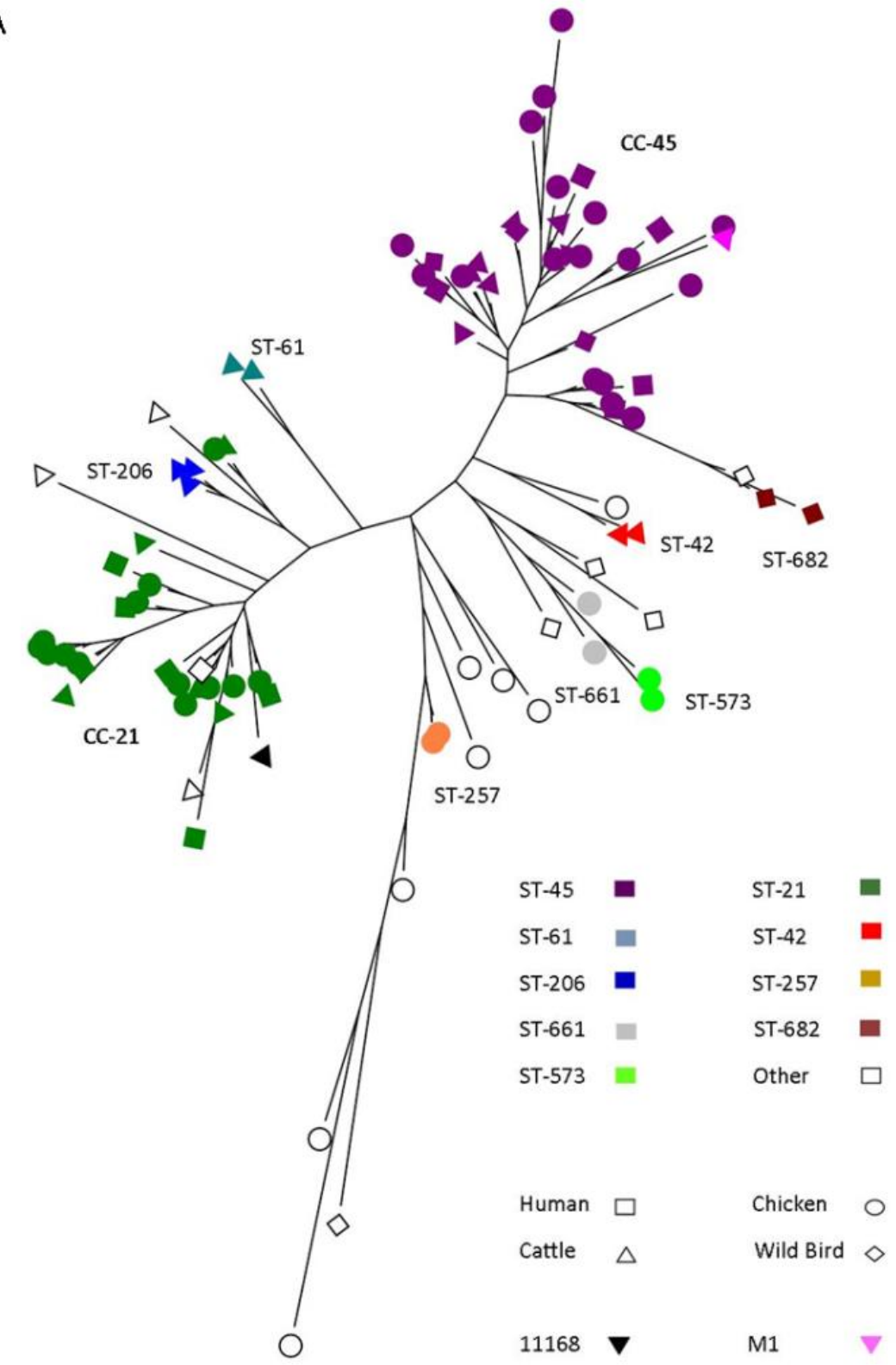

Figure 3.2. Phylogenetic tree of the pan genome of the $C$. jejuni from 100 isolates taken from a variety of sources with two reference strains, 11168 and M1 included. 11168 and M1 cluster within the two main clonal complexes CC-21 and CC-45, respectively. 

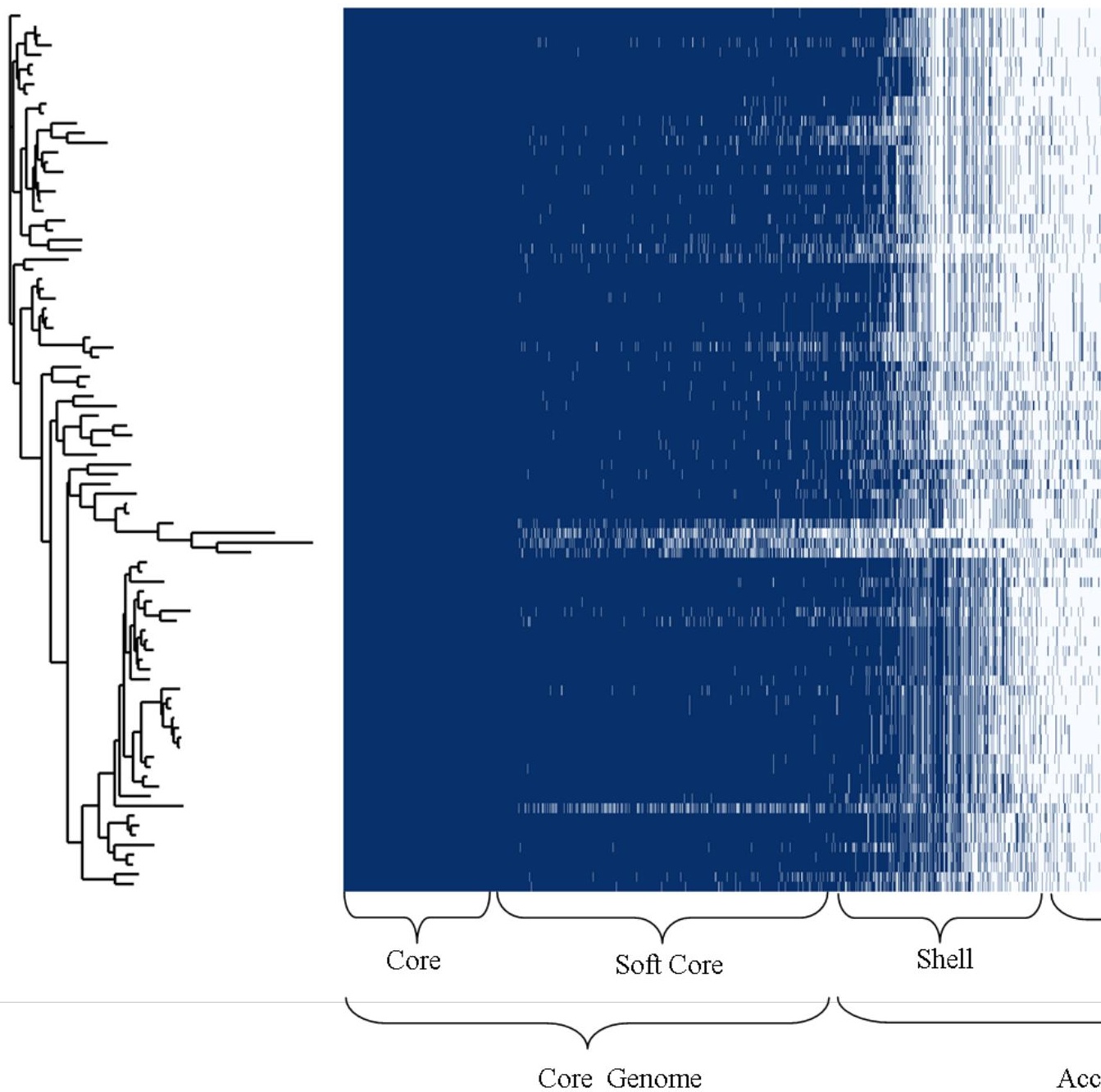

Figure 3.3. Representation of the pan-genome showing the core and accessory genome with relatio genome is made up of the Core $(99 \%<=$ gene presence $<=100 \%)$, and the soft core $(95 \%<=$ gene accessory genome is made up of the shell $(15 \%<=$ gene presence $<=95 \%)$ and the cloud genes $(0 \%$ 


\subsubsection{Core vs. Accessory Genome}

The core genome of the 100 C. jejuni isolates analysed totalled 1135 genes which included core and soft-core ones. Of these genes, 246 were found to be hypothetical proteins and were removed from further analysis, as no credible information could be drawn from them. The accessory genome totalled 1580 genes in both the shell and cloud, of which 808 were hypothetical proteins and removed from further analysis (Figure 3.3).

Analysis of known virulence genes (Table 3.1) demonstrated the presence of the motility genes $f l g S$ and $f l g R$ in the core genome with $f l a C$ located in the accessory genome. Two adhesion linked genes; pebAl and $p l d A$, were found in the core genome with all others being present in the accessory genome (Table 3.2). All $c d t$ toxicity genes were in the accessory genome whereas the invasion linked genes ciaB, ciaC and ciaI were all found within the core. The remaining invasion-linked genes, $h \operatorname{trA}$, iamA and $f_{s p A}$, were within the accessory genome (Table 3.2). All known antimicrobial resistance genes were in the accessory genome including $c m e B$, aadE, tet $(O), b_{O X A-61}$ and $a p h-3-$ I. 
Table 3.2. Presence of known virulence linked genes in the core and accessory genome

\begin{tabular}{|c|c|c|c|}
\hline Gene & Name/Function & Core & Accessory \\
\hline flaA & Flagellin A & & $\mathrm{x}$ \\
\hline flaB & Flagellin B & & $\mathrm{x}$ \\
\hline flaC & Flagellin C & & $\mathrm{x}$ \\
\hline$f \lg S$ & Signal transduction histidine kinase & $\mathrm{x}$ & \\
\hline$f \lg R$ & $\begin{array}{l}\text { Sigma-54 associated transcriptional } \\
\text { activator }\end{array}$ & $\mathrm{x}$ & \\
\hline$c a d F$ & $\begin{array}{l}\text { Outer membrane fibronectin-binding } \\
\text { protein }\end{array}$ & & $\mathrm{x}$ \\
\hline capA & Auto-transporter protein & & $\mathrm{x}$ \\
\hline pebAl & $\begin{array}{l}\text { Bi-functional adhesion/ABC transporter } \\
\text { aspartate/ glutamate-binding protein }\end{array}$ & $\mathrm{x}$ & \\
\hline peb3 & Major antigenic peptide PEB3 & & $\mathrm{x}$ \\
\hline peb4 & Peptidyl-prolyl cis-trans isomerase & & $\mathrm{x}$ \\
\hline pldA & phospholipase A & $\mathrm{x}$ & \\
\hline$j l p A$ & Surface exposed lipoprotein & & $\mathrm{x}$ \\
\hline$c i a B$ & Campylobacter invasion antigen B & $\mathrm{x}$ & \\
\hline $\operatorname{ciaC}$ & Campylobacter invasion antigen $\mathrm{C}$ & $\mathrm{x}$ & \\
\hline ciaI & Campylobacter invasion antigen I & $\mathrm{x}$ & \\
\hline htrA & Serine protease & & $\mathrm{x}$ \\
\hline $\operatorname{iamA}$ & $\mathrm{ABC}$ transporter ATP-binding protein & & $\mathrm{x}$ \\
\hline$f s p A$ & flagellum-secreted non flagellar protein & & $\mathrm{x}$ \\
\hline$c d t A$ & Cytolethal distending toxin $\mathrm{A}$ & & $\mathrm{x}$ \\
\hline$c d t B$ & Cytolethal distending toxin B & & $\mathrm{x}$ \\
\hline$c d t C$ & Cytolethal distending toxin $\mathrm{C}$ & & $\mathrm{x}$ \\
\hline
\end{tabular}




\subsubsection{Analysis of $C$. jejuni virulence factors in the accessory genome}

The presence of known virulence genes found in the accessory genome (Table 3.2) of the C. jejuni strains were analysed using gene-by-gene analysis (Figure 3.4 and Table 3.3). The $f l a A / B$ genes showed the lowest presence across the 100 isolates, compared to all other genes investigated, with only $23 \%$ of all genomes containing flaA/B. All three $c d t$ genes were present in almost all human, chicken and cattle strains. Isolates from wild birds had a lower presence of $c d t A$, with it only being present in $60 \%$ of strains compared to $90 \%$ with both $c d t B$ and $c d t C$. No association was found when comparing genes present in the accessory genome and source of $C$. jejuni isolate.

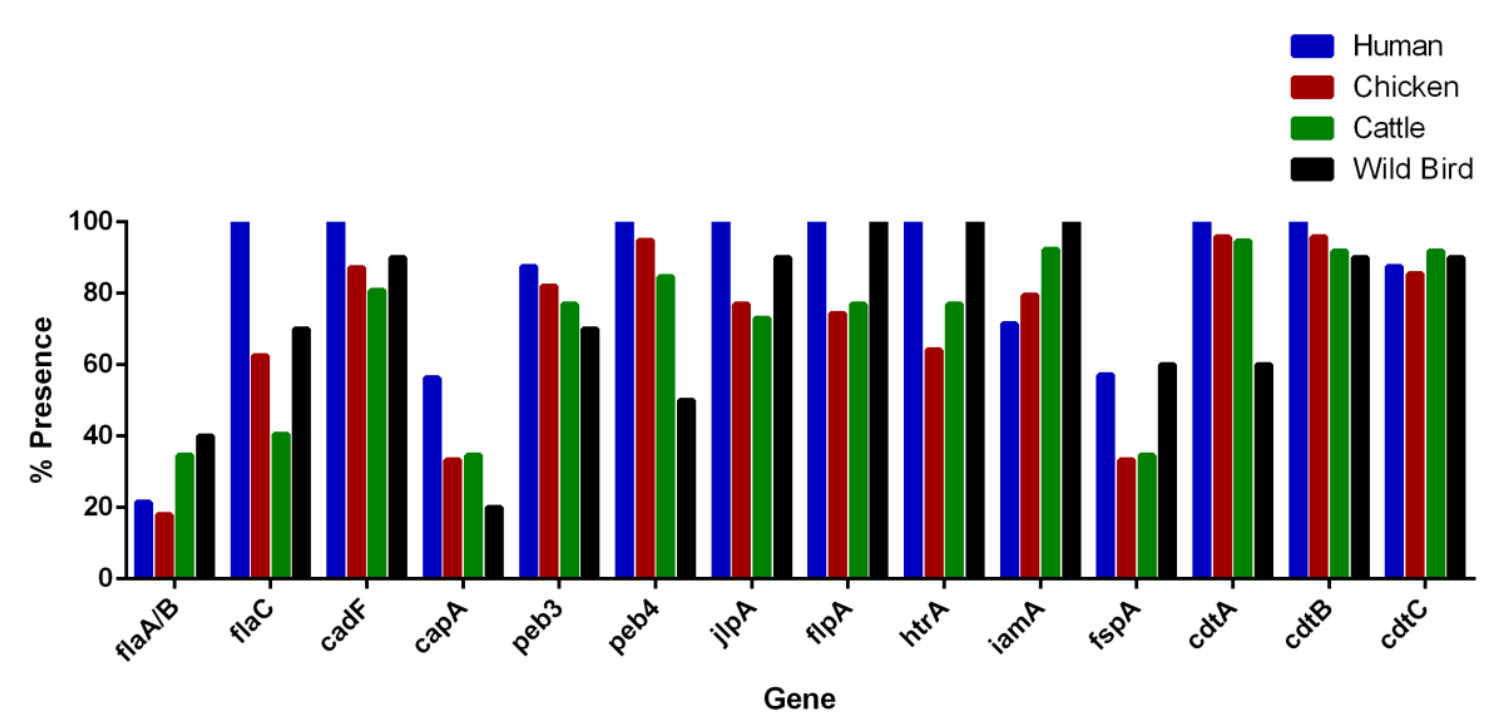

Figure 3.4. Presence of known virulence genes from the accessory genome in strains isolated from human, chicken, cattle and wild bird sources. 
Table 3.3. Presence of known virulence genes in the core and accessory genome of $C$. jejuni populations

\begin{tabular}{|c|c|c|c|c|c|}
\hline \multirow[t]{2}{*}{ Gene } & \multicolumn{4}{|c|}{ Gene Presence (\%) } & \multirow{2}{*}{$\begin{array}{l}\text { Presence within core genome } \\
(\%)\end{array}$} \\
\hline & Human & Chicken & Cattle & Wild Bird & \\
\hline$f \lg S$ & 100.00 & 100.00 & 100.00 & 100.00 & 100.00 \\
\hline$f l g R$ & 100.00 & 100.00 & 100.00 & 100.00 & 100.00 \\
\hline pebA1 & 100.00 & 100.00 & 100.00 & 100.00 & 100.00 \\
\hline pldA & 100.00 & 100.00 & 100.00 & 100.00 & 100.00 \\
\hline$c i a B$ & 100.00 & 100.00 & 100.00 & 100.00 & 100.00 \\
\hline ciaC & 100.00 & 100.00 & 100.00 & 100.00 & 100.00 \\
\hline ciaI & 100.00 & 100.00 & 100.00 & 100.00 & 100.00 \\
\hline & & & & & $\begin{array}{l}\text { Presence within accessory } \\
\text { genome }(\%)\end{array}$ \\
\hline$f l a A / B$ & 21.43 & 17.95 & 34.62 & 40.00 & 23 \\
\hline flaC & 100.00 & 62.50 & 40.54 & 70.00 & 66 \\
\hline$c a d F$ & 100.00 & 87.18 & 80.77 & 90.00 & 78 \\
\hline $\operatorname{cap} A$ & 56.25 & 33.33 & 34.62 & 20.00 & 33 \\
\hline peb3 & 87.50 & 82.05 & 76.92 & 70.00 & 71 \\
\hline peb4 & 100.00 & 94.87 & 84.62 & 50.00 & 78 \\
\hline$j l p A$ & 100.00 & 76.92 & 73.08 & 90.00 & 72 \\
\hline$f l p A$ & 100.00 & 74.36 & 76.92 & 100.00 & 73 \\
\hline htrA & 100.00 & 64.10 & 76.92 & 100.00 & 69 \\
\hline $\operatorname{iam} A$ & 71.43 & 79.49 & 92.31 & 100.00 & 75 \\
\hline$f s p A$ & 57.14 & 33.33 & 34.62 & 60.00 & 36 \\
\hline$c d t A$ & 100.00 & 95.83 & 94.59 & 70.00 & 86 \\
\hline$c d t B$ & 100.00 & 95.83 & 91.83 & 90.00 & 91 \\
\hline$c d t C$ & 87.50 & 85.42 & 91.89 & 90.00 & 88 \\
\hline
\end{tabular}

\subsubsection{GWAS of $C$. jejuni strains by source}

Scoary was used to investigate association between gene presence/absence and source of the $C$ jejuni isolates. Scoary compared isolates from one particular source to every other source. For example, human isolates were compared to chicken, cattle and wild 
bird ones. Scoary found 22 genes linked specifically to human isolates, excluding 15 hypothetical proteins. Genes related to human isolates had a variety of characteristics including lipoproteins ( $(\mathrm{lp} A)$, heme related (hemH), flagellar (flgE), RNA degradation $(r p p H)$ and transporter proteins (proP) (Table 3.4). The genes rppH and hemH were significantly related to human isolates being found to be trait positive in 13 of the 16 human strains, both yielding p values of 0.02 (Table 3.4). All 22 genes found were significantly related to human isolates (Appendix Table 8.3).

Scoary identified 27 genes linked to chicken isolates, excluding 23 hypothetical proteins. Among those related to chicken sources were genes encoding for Sigma factor 54 transcriptional activator $(f l g R)$, drug resistance proteins $(C j 1375)$, iron transporters $(f e o B)$, exporters $(y m f D)$ and transferases $(m u r G)$ (Table 3.5). The gene murG was traitpositive in 40 of the 42 chicken isolates and significantly related with $(\mathrm{p}=0.01)$. The genes $n t r C$ and $y k k C$ were trait positive in 39 and 34 chicken isolates, respectively. All 27 genes found were significantly related to the chicken trait (Appendix, Table 8.4).

Table 3.4. Top genes (highest trait positive scores) found to be associated with strains isolated from humans.

\begin{tabular}{|c|c|c|c|c|}
\hline Gene & Locus Tag & Annotation & $\begin{array}{l}\text { Trait Positive } \\
\text { Isolates }\end{array}$ & p Value \\
\hline$r p p H$ & $\mathrm{Cj} 0581$ & RNA pyrophosphohydrolase & 13 & 0.026217 \\
\hline hemH & $\mathrm{Cj} 0503 \mathrm{c}$ & Ferrochelatase & 13 & 0.026217 \\
\hline$n \operatorname{lp} A$ & $\mathrm{Cj0771c}$ & Lipoprotein 28 & 12 & 0.031127 \\
\hline patA & Cj0639 & $\begin{array}{ll}\text { Peptidoglycan } & \text { O- } \\
\text { acetyltransferase } & \end{array}$ & 11 & 0.006454 \\
\hline$d s b L$ & $\mathrm{Cj0865}$ & $\begin{array}{l}\text { Thiol:disulfide interchange } \\
\text { protein DsbL }\end{array}$ & 11 & 0.024205 \\
\hline $\operatorname{clp} A$ & $\mathrm{Cj} 1108$ & $\begin{array}{l}\text { ATP-dependent Clp protease } \\
\text { ATP-binding subunit ClpA }\end{array}$ & 11 & 0.024205 \\
\hline proP & Cj0339 & Proline/betaine transporter & 10 & 0.010203 \\
\hline$f \operatorname{flg} E$ & $\mathrm{Cj} 0043$ & Flagellar hook protein FlgE & 8 & 0.005657 \\
\hline aldA & Cj1548c & Lactaldehyde dehydrogenase & 7 & 0.006670416 \\
\hline$h d d C$ & Cj1431c & $\begin{array}{l}\text { D-glycero-alpha-D-manno- } \\
\text { heptose 1-phosphate } \\
\text { guanylyltransferase }\end{array}$ & 7 & 0.035565058 \\
\hline
\end{tabular}


Table 3.5. Top genes (highest trait positive scores) found to be associated with strains isolated from chickens.

\begin{tabular}{|l|l|l|l|l|}
\hline Gene & Locus Tag & Annotation & $\begin{array}{l}\text { Trait Positive } \\
\text { Isolates }\end{array}$ & p Value \\
\hline murG & $\mathrm{Cj} 1039$ & $\begin{array}{l}\text { UDP-N-acetylglucosamine--N- } \\
\text { acetylmuramyl-(pentapeptide) } \\
\text { pyrophosphoryl-undecaprenol } \\
\text { N-acetylglucosamine transferase }\end{array}$ & 40 & 0.015864 \\
\hline flgR & $\mathrm{Cj} 024 \mathrm{c}$ & $\begin{array}{l}\text { Sigma-54 associated } \\
\text { transcriptional activator }\end{array}$ & 39 & 0.039786 \\
\hline Cj1375 & $\mathrm{Cj} 1375$ & Multidrug efflux transporter & 34 & 0.041552 \\
\hline grpE & $\mathrm{Cj} 0758$ & Protein GrpE & 32 & 0.000992 \\
\hline ymfD & $\mathrm{Cj} 1687$ & Bacillibactin exporter & 31 & 0.015862 \\
\hline feoB & $\mathrm{Cj} 1398$ & Ferrous iron transport protein B & 29 & 0.000442 \\
\hline torC & $\mathrm{Cj} 0265 \mathrm{c}$ & Cytochrome c-type protein TorC & 21 & 0.006902 \\
\hline metC & $\mathrm{Cj} 1392$ & Cystathionine beta-luase & 15 & 0.001326 \\
\hline porA & $\mathrm{Cj} 1259$ & Major outer membrane protein & 9 & 0.004334 \\
\hline
\end{tabular}

Scoary identified 41 genes related to cattle isolates, excluding 30 hypothetical proteins. Among those from cattle sources were genes encoding for flagellar associated gene (fldD), iron transporter ( $f e o B)$, and a histidine binding (hisJ) (Table 3.6). The genes flgD and $f e o B$ were found to be trait positive in 25 of the 31 cattle isolates and significantly related with $\mathrm{p}$ values of 0.03 . All 41 genes found were significantly related to the cattle trait (Appendix, Table 8.5).

Scoary revealed 90 genes related to wild bird isolates, excluding 82 hypothetical proteins. The genes found to be trait positive with the highest frequency included ones encoding for a DNA ligase (ligA), bacterial haemoglobin $(c g b)$ and sensor proteins $(c j 1492 c)$. Within 9 of the 10 wild bird isolates the genes $h r c A$ and $h y d A$ were found to be trait positive with significant associations ( $\mathrm{p}$ values of 0.002 and 0.004 , respectively; Table 3.7). All 90 genes found were significantly related to the wild bird trait (Appendix Table 8.6). 
Table 3.6. Top genes (highest trait positive scores) found to be associated with strains isolated from cattle.

\begin{tabular}{|l|l|l|l|l|}
\hline Gene & Locus Tag & Annotation & $\begin{array}{l}\text { Trait Positive } \\
\text { Isolates }\end{array}$ & p Value \\
\hline flgD & $\mathrm{Cj0042}$ & $\begin{array}{l}\text { Basal-body rod modification } \\
\text { protein FlgD }\end{array}$ & 25 & 0.030579 \\
\hline pyrC & $\mathrm{Cj0259}$ & Dihydroorotase & 25 & 0.030579 \\
\hline feoB & $\mathrm{Cj} 1398$ & Ferrous iron transport protein B & 25 & 0.032227 \\
\hline panB & $\mathrm{Cj0298c}$ & $\begin{array}{l}\text { 3-methyl-2-oxobutanoate } \\
\text { hydroxymethyltransferase }\end{array}$ & 24 & 0.000306 \\
\hline$d g k A$ & $\mathrm{Cj0257}$ & Diacylglycerol kinase & 24 & 0.005679 \\
\hline panC & $\mathrm{Cj0297c}$ & Pantothenate synthetase & 23 & 0.002187 \\
\hline panD & $\mathrm{Cj0296c}$ & Aspartate 1-decarboxylase & 23 & 0.002187 \\
\hline hisJ & $\mathrm{Cj0734c}$ & Histidine-binding protein & 22 & 0.011435 \\
\hline fabD & $\mathrm{Cj0116}$ & $\begin{array}{l}\text { Malonyl CoA-acyl carrier } \\
\text { protein transacylase }\end{array}$ & 22 & 0.019578 \\
\hline
\end{tabular}

Table 3.7. Top genes (highest trait positive scores) found to be associated with strains isolated from wild birds.

\begin{tabular}{|c|c|c|c|c|}
\hline Gene & Locus Tag & Annotation & $\begin{array}{ll}\text { Trait } & \text { Positive } \\
\text { Isolates } & \end{array}$ & p Value \\
\hline$h r c A$ & $\mathrm{Cj} 0757$ & $\begin{array}{l}\text { Heat-inducible transcription } \\
\text { repressor HrcA }\end{array}$ & 9 & 0.002728 \\
\hline hydA & Cj1267c & $\begin{array}{l}\text { Quinone-reactive Ni/Fe- } \\
\text { hydrogenase small chain }\end{array}$ & 9 & 0.004608 \\
\hline$c j 1492 c$ & Cj1492c & Sensor protein & 8 & 0.009051 \\
\hline $\operatorname{lig} A$ & Cj0586 & DNA ligase & 8 & 0.029978 \\
\hline$h d d A$ & $\mathrm{Cj} 1425 \mathrm{c}$ & $\begin{array}{l}\text { D-glycero-alpha-D-manno- } \\
\text { heptose 7-phosphate kinase }\end{array}$ & 8 & 0.033468 \\
\hline$g m h A$ & Cj1149c & Phosphoheptose isomerase & 8 & 0.036215 \\
\hline yajR & $\mathrm{Cj} 1241$ & $\begin{array}{l}\text { Inner membrane transport } \\
\text { protein YajR }\end{array}$ & 7 & 0.025536 \\
\hline
\end{tabular}




\begin{tabular}{|l|l|l|l|l|}
\hline$c g b$ & $\mathrm{Cj} 1586$ & Bacterial hemoglobin & 7 & 0.025536 \\
\hline$f d h C$ & $\mathrm{Cj} 1509 \mathrm{c}$ & $\begin{array}{l}\text { Formate dehydrogenase, } \\
\text { cytochrome b556(fdo) } \\
\text { subunit }\end{array}$ & 7 & 0.025536 \\
\hline CarB & Cj0279 & $\begin{array}{l}\text { Carbmoyl-phosphate } \\
\text { sythase large chain }\end{array}$ & 7 & 0.04836 \\
\hline
\end{tabular}




\subsubsection{Recombination in $C$. jejuni isolates}

Through the use of Gubbins (Croucher et al., 2015) a total of 39,916 SNPs were detected in the isolates and 25,091 of these were outside recombination regions and so excluded from further analysis. A total of 14,825 SNPs were detected within recombination regions. Most of the regions detected were shared by multiple isolates (red regions) (Figure 3.5). However, there were unique recombination sites identified in many isolates (blue regions) (Figure 3.5). Using Gubbins, the rate of nucleotide change caused by recombination was measured, relative mutation in ST-45 strains $(\mathrm{r} / \mathrm{m}=2.82)$ occurred at a higher rate compared to relative mutation in ST-21 strains $(\mathrm{r} / \mathrm{m}=1.47)$. Variation was also observed in recombination estimated from different sources of $C$. jejuni. Relative mutation rate in isolates taken from human sources $(\mathrm{r} / \mathrm{m}=5.97)$ occurred at a much higher rate compared to chicken $(\mathrm{r} / \mathrm{m}=3.86)$, cattle $(\mathrm{r} / \mathrm{m}=4.92)$ and wild bird isolates $(\mathrm{r} / \mathrm{m}=2.48)$. 

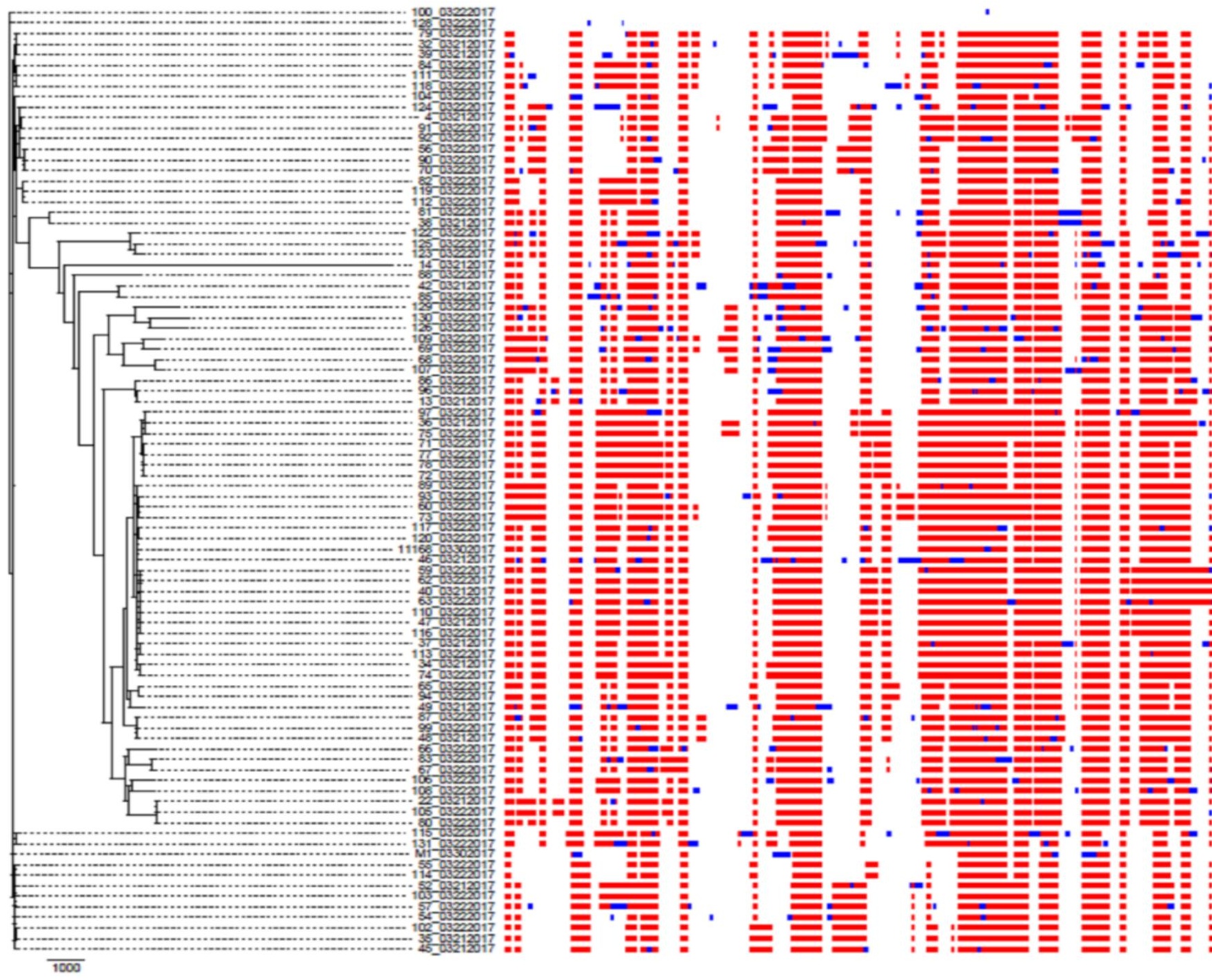

Figure 3.5. Representation of recombination regions present within the 100 Campylobacter genomes analy regions shared with two or more isolates while blue regions denote unique recombination regions to an indi 


\subsubsection{Growth of $C$. jejuni}

The $100 C$. jejuni isolates were subjected to growth assays under varying conditions. They were grown at 37 and $42^{\circ} \mathrm{C}$ in a microaerobic environment (Figure 3.6a), where strains grown at $42^{\circ} \mathrm{C}$ grew at a significantly $(\mathrm{p}<0.05)$ faster rate and to a significantly ( $p<0.05$ ) higher population density than ones grown at $37^{\circ} \mathrm{C}$. A similar trend was observed when strains were grown under normal atmospheric conditions albeit to a lower extent (Figure 3.6b) and no significant difference $(\mathrm{p}>0.05)$ between strains grown at 37 and $42^{\circ} \mathrm{C}$ was observed. All 100 strains from the various sources grew at similar rates.

a

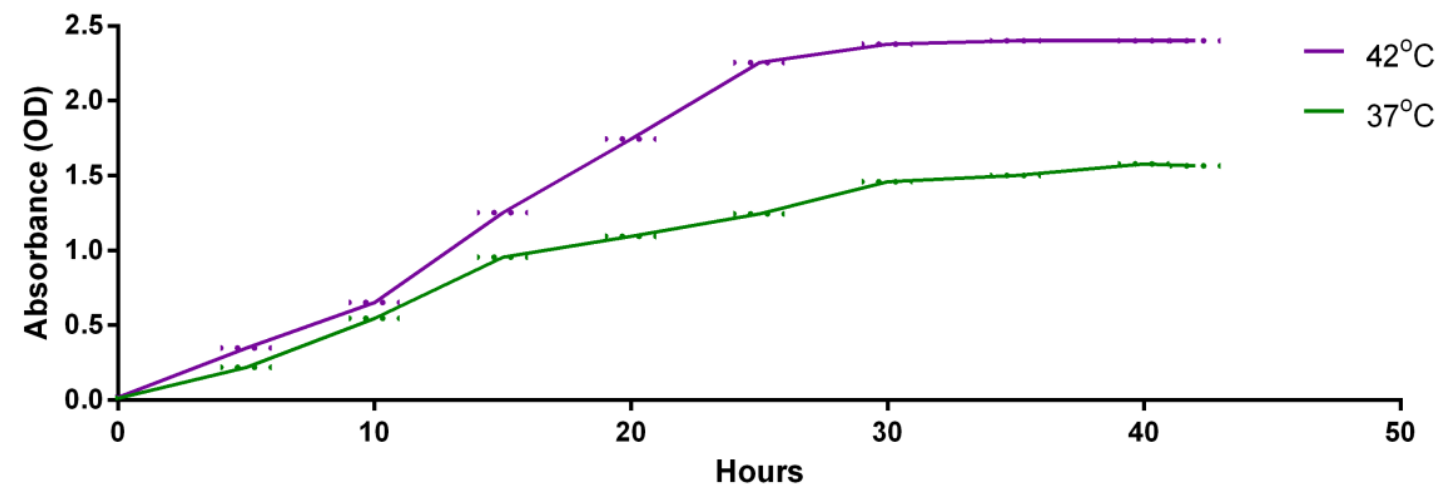

b

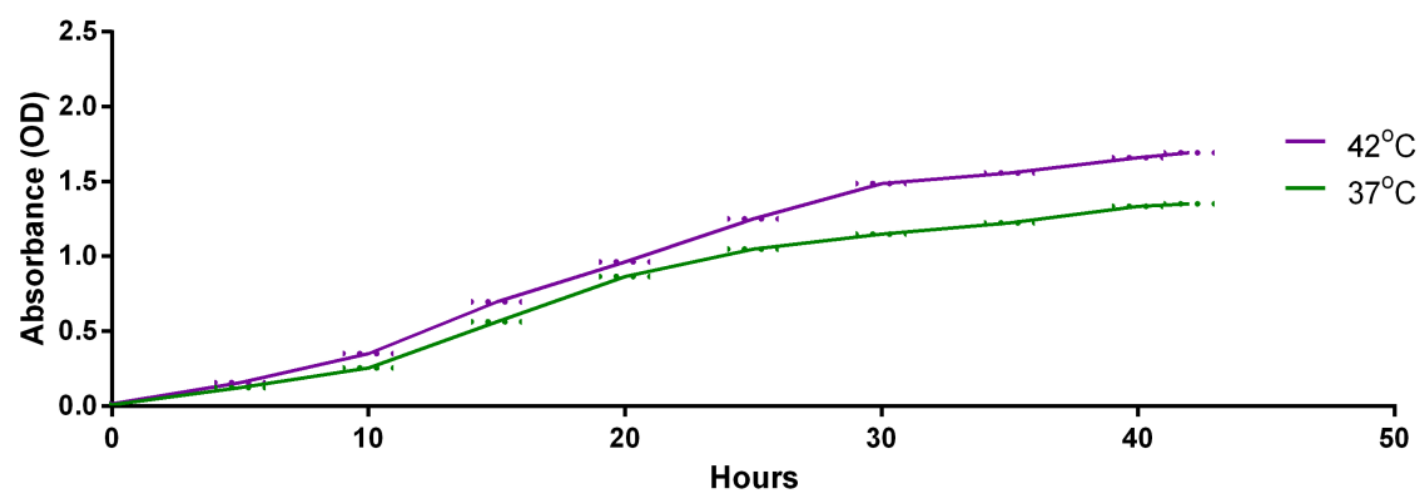

Figure 3.6. Growth of Campylobacter jejuni $(n=100)$ strains under varying conditions. a) Strains subjected to a microaerophilic environment at $37^{\circ} \mathrm{C}$ and $42^{\circ} \mathrm{C}$; b) strains subjected to normal atmospheric conditions at $37^{\circ} \mathrm{C}$ and $42^{\circ} \mathrm{C}$. 


\subsubsection{Biofilm Formation}

A $25 \%$ sub-group of $C$. jejuni isolates categorised by presence of the genes $f l a A / B$ as well as strains from a variety of STs (Appendix table 8.1) were used to test the ability of the strains to form biofilm using analysis with crystal violet, with an absorbance $(570 \mathrm{~nm})$ of greater than 0.1 defined as biofilm positive. The $C$. jejuni strains grown at $42^{\circ} \mathrm{C}$ were shown to form the strongest biofilms in a microaerobic atmosphere (Figure 3.7a) with a significant difference $(\mathrm{p} \leq 0.01)$ shown between strains grown at this temperature and at $37^{\circ} \mathrm{C}$. Under normal atmospheric conditions strains grown at $42^{\circ} \mathrm{C}$ displayed a trend towards stronger biofilms but results did not reach significance (Figure 3.7b). C. jejuni strains grown in a microaerobic atmosphere produced stronger biofilms at both temperatures (Figure 3.7); with significantly stronger biofilms formed at $42^{\circ} \mathrm{C}$ under microaerobic conditions $(\mathrm{p} \leq 0.01)$. No differences were observed between biofilms formed by strains from difference sources at either temperature (Figure 13cd).

a

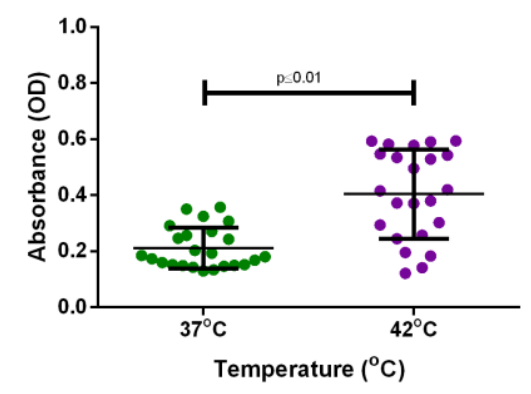

C

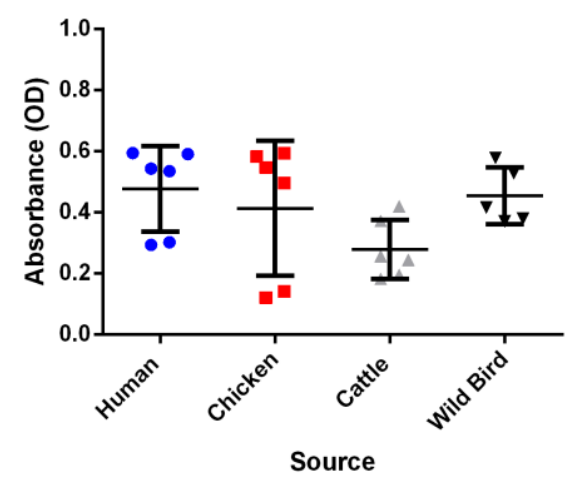

b

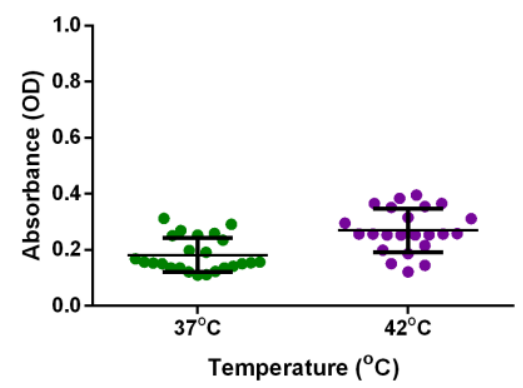

d

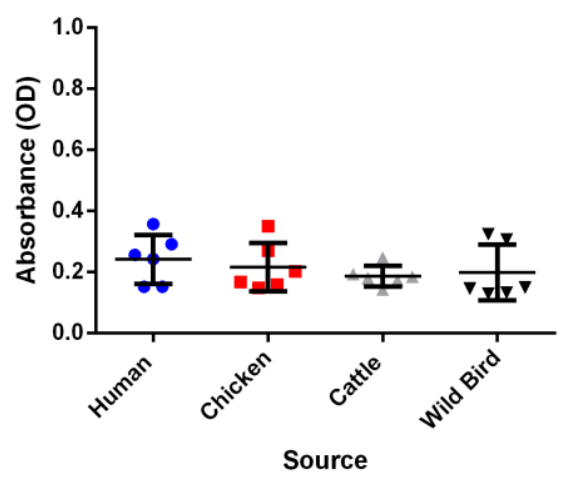

Figure 3.7. Effect of growth atmosphere and temperature on $C$. jejuni biofilm formation analysed through crsytal violet. C. jejuni strains were grown at 37 and $42^{\circ} \mathrm{C}$ under microaerophilic conditions (a) and under normal atmospheric conditions (b), with biofilm organised by source of $C$. jejuni strain at 42 (c) and $37{ }^{\circ} \mathrm{C}$ (d). Results are also expressed as mean $+/-\mathrm{SD}$ of all strains measured. Differences were considered significant if $\mathrm{p} \leq 0.05$. 


\subsubsection{Motility of $C$. jejuni strains}

The motility of a $25 \%$ sub group of $C$. jejuni isolates categorised by presence of the genes $f l a A / B$ as well as strains from a variety of STs (Appendix table 8.1) was measured with isolates from human sources found to have the greatest motility compared to strains from chickens, cattle and wild birds. Isolates from chickens showed the lowest motility overall; a significant difference was observed between human and chicken isolates ( $\mathrm{p} \leq 0.05$; Figure 3.8). Isolates that were $f l a A / B$ positive (shown in green on Figure 3.8) usually had the highest rates of motility compared to isolates which were flaA $B$ negative; with a significant difference $(\mathrm{p} \leq 0.05)$ seen when $f l a A / B$ positive isolates were compared to the $f l a A / B$ negative isolates (Figure 3.8).
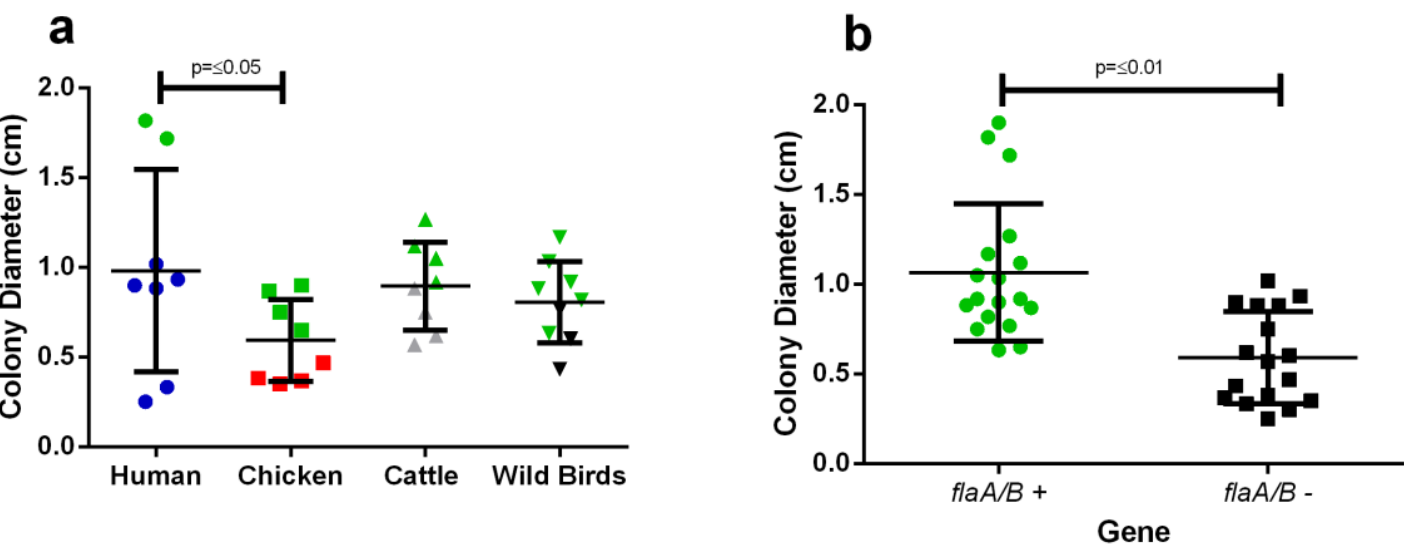

Figure 3.8. Motility assay performed on a $25 \%$ sub-group of $C$. jejuni isolates from (a) different sources and (b) motility in regards to the presence of flaA and flaB genes . Isolates coloured green represent isolates which are $f l a A / B$ positive. Results are also expressed as mean +/- SD of all strains measured. Differences were considered significant if $\mathrm{p} \leq 0.05$. 


\subsection{Discussion}

A population of $C$. jejuni isolates from a variety of different sources and sequence types was analysed. MLST analysis of the 100 C. jejuni strains displayed two distinct clusters of clonal complexes ST-21 and -45 in the neighbour joining tree. These are the two most common $C$. jejuni clonal complexes reported and this grouping is to be expected and supports previous publications (Méric et al., 2014; Sheppard et al., 2008). MLST analysis of $C$. jejuni isolates provided a good initial phylogenetic analysis of the dataset in conjunction with the Campylobacter MLST database (http://pubmlst.org/campylobacter/) which contains over 60,000 isolates as of June 2018 and is widely used by Campylobacter researchers (Dingle et al., 2001; Jolley and Maiden, 2010; Jorgensen et al., 2011; Kittl et al., 2013; Sheppard et al., 2008). Whilst MLST characterised the phylogeny it could not answer questions on the presence or absence of key virulence genes amongst the strains and so further genomic identification was necessary.

Creation of the $C$. jejuni pan-genome which used $C$. jejuni NCTC 11168 as a reference strain resulted in one containing 2715 genes; of which 1135 were present in the core genome. Roary was run with a $95 \%$ cut off for the core genome to create a pan-genome of only the most essential genes found in $C$. jejuni. $C$. jejuni NCTC 11168 was used, as it remains a well-characterised and widely used reference strain in Campylobacter studies (Dingle et al., 2001; Hall et al., 2010; Koolman et al., 2016; Sheppard et al., 2012) and was the first $C$. jejuni strain to have its whole genome sequenced and annotated (Parkhill et al., 2000). The pan-genome consisted of C. jejuni isolates only and so is relatively small compared to other Campylobacter pan-genomes previously created using both $C$. jejuni and $C$. coli (Lefebure et al., 2010; Méric et al., 2014). Creation of a maximum likelihood tree from the pan-genome resulted in a similar phylogenetic tree to the one created using MLST data with the characteristic clusters of clonal complexes ST- 45 and -21 on either end of the tree. Pan-genome approaches to genome analysis provide a much higher strain typing resolution than MLST, allowing discrimination among many bacterial strains within a species (Hall et al., 2010). The pan-genome was created using Prokka and Roary (Page et al., 2015; Seemann, 2014), and through the use of this software a more up to date view of the population of $C$. jejuni has been created and thus provides a clearer and more in-depth analysis of the 
population as the software has been demonstrated high speed applications to construct pan genomes with many third party analysis tools using Roary results which can be integrated for further analysis and visualisation (Yu et al., 2017).

Gene by gene analysis of known virulence genes in the sequences yielded large differences in $f l a A / B$, flaC and $c d t A$. The $f l a A / B$ genes were absent in $71.05 \%$ of the strains analysed which led to the phenotypic analysis of the motility of the isolates however this lack of $f l a A / B$ could be due to variation within the genes and further analysis of the allelic differences of the fla genes could provide invauable insight. The apparent absence of $f l a A / B$ did not seem to affect motility with all strains being motile. However, the absence of these genes may affect the ability of $C$. jejuni to invade into epithelial cells as flaA is essential for invasion into such cells and responsible for adherence to them (Jain et al., 2008). The absence of flaC may also effect invasion and adhesion as Faber et al., found that flaC is a secreted effector flagellin which may help to modulate the immune response and invasion into epithelial cells (Faber et al., 2015). The $c d t A$ gene was present in almost all strains isolated from humans, chickens and cattle; however, there was a marked decrease of $c d t A$ presence in the strains isolated from wild birds, which warranted more attention. This gene encodes for cytolethal distending toxin A, one part of three subunits of CDT which causes DNA damage and leads to cell death (Lee et al., 2003). The lack of $c d t A$ could prevent the correct formation of CDT in these strains resulting in lower toxicity to host cells. Indeed this was investigated in later work described in Chapter 4 with human and chicken epithelial cells. All CDT genes, $c d t A, c d t B$ and $c d t C$, were found in the accessory genome. The presence of these genes throughout the isolates may contribute to the overall severity of C. jejuni strains during infection with strains lacking $c d t$ genes being less harmful to a host compared to strains with the ability to produce CDT and cause toxicity in a host which supports work by Jain et al where $\mathrm{CDT}^{+}$strains of $C$. jejuni were more effectively adhered and invaded into epithelial cells compared to $\mathrm{CDT}^{-}$ones (Jain et al., 2008).

Analysis of genes present in the core and accessory genome yielded the presence of some interesting known virulence genes. The motility genes flaA and flaB were present within the accessory genes, both fla genes being present in the same genome is to be expected as $f l a A$ and $f l a B$ are gene duplications that have specialised. This is a good example of one of the major mechanism of gene evolution (Meinersmann and Hiett, 2000). The $f \lg S$ and $f \lg R$ genes which are responsible for flagellar biosynthesis were 
also present in the core genome which form Campylobacter flagellin. It is no surprise that these genes were present in most $C$. jejuni strains as they provide functional motility and promote colonisation and survival as mutational studies of flagella have found that flagella appear to be required for maximal biofilm formation (Guerry, 2007; Joslin and Hendrixson, 2009; Reeser et al., 2007). The Campylobacter invasion antigenassociated genes, $c i a B$, ciaC and cial, were all present within the core genome. The cia genes have been known to help invasiveness and intraceullular survival of $C$. jejuni (Buelow et al., 2011; Koolman et al., 2016; Neal-McKinney and Konkel, 2012). The Cia proteins also use flagellar export apparatus and so the presence of these genes and the $f l a$ and $f l g$ genes in the core genome may help $C$. jejuni to survive and invade into host cells (Konkel et al., 2004). All antimicrobial resistance genes were found in the accessory genome; suggesting strains with unique resistance profiles. This is consistent with studies confirming that resistance is on the rise over the last decade (Bardon et al., 2017; Guévremont et al., 2006; Obeng et al., 2012; Ruiz-Palacios, 2007).

Using Scoary, differences in genes related to the source of the isolate were identified. Wild bird isolates had 90 genes closely related to the trait. The cause of such a high number of genes being linked to this trait could be due to wild birds having less contact with other reservoirs of $C$. jejuni compared to cattle and poultry and so have evolved into a separate niche (Griekspoor et al., 2013; Sheppard et al., 2011). Another possible explanation could be due to the wild bird isolates encompassing many different species including geese, ducks and starling. We might speculate that each of these birds will have different gut environments and Campylobacter found in them may have their own specific genes essential for niche adaption.

The 22 genes were related to human isolates including: $h d d C$, a heptose transferase, which is related to the polysaccharide capsule an interface between the bacteria and the environment and so may contribute to the virulence of human strains (Poly et al., 2011). Another gene, $f \lg E$, which encodes for the flagellar hook protein FlgE was also found to be related to human isolates which may contribute to motility and thus their virulence . Matsunami et al (2016) showed that the protein FlgE in C. jejuni provides stability and robustness of the hook which helps survival of $C$. jejuni thus the human isolates may have a better chance of survival compared to strains from chickens, cattle and wild birds (Matsunami et al., 2016). 
Strains from chickens had 27 genes related to the chicken source including $f l g R$, a sigma-54 associated transcriptional activator and $C j 1375$, a multidrug efflux transporter, which could aid the persistence of $C$. jejuni in chickens, particulalry if they are treated with antibiotics. The motility linked $f \lg R, a$ NtrC-like protein which are a family of transcriptional activators and Joslin et al (2008) found that flagellar motility, specifically FlgR activation mechanisms, are utilised to control the transcription of target genes. Multiple studies have shown that non-motile isolates are less invasive and thus the ability to control flagella more efficiently could give a large advantage to $C$. jejuni isolates (Baldvinsson et al., 2014; Golden and Acheson, 2002; O Cróinín and Backert, 2012). Thus, I might suggest that strains isolated from chicken may be able to utilise FlgR activation mechanisms to control flagellar motility more efficiently, and this could help $C$. jejuni migration from the gut and enter other organs such as the liver (Jagannathan et al., 2000; Joslin and Hendrixson, 2008; Reid et al., 2016).

Scoary identified 41 genes related to strains isolated from cattle. Among these was gene $f e o B$, which encodes for a ferrous iron transporter protein which Naikare et al (2006) showed to have a major role in the acquisition, gut colonisation and persistence, which may help to explain the ability of $C$. jejuni to persist in cattle. These animals possess a complex four compartment stomach, small intestine and large intestine and so acquisition and regulation of iron by $C$. jejuni may help contribute to survival (Palyada et al., 2004).

High rates of recombination due to mutations were found in the isolates analysed. Interestingly, the normal rate of recombination in $C$. jejuni has been estimated to be $\mathrm{r} / \mathrm{m}$ $=2.2$ (Vos and Didelot, 2009). Recombination rates between the different sources may help to explain the differences seen between different source lineages when a Campylobacter lineage transfers to a new animal host it could acquire DNA from the resident population through horizontal gene transfer. This has been observed by McCarthy et al (2007) and Wilson et al (2008) where C. jejuni lineages isolated from chicken contained alleles that originated in chicken specialist genotypes. These recombination rates are much higher than those of other bacteria such as Staphylococcus aureus and Escherichia coli which have very low rates of $\mathrm{r} / \mathrm{m}=0.1$ and 0.7 , respectively. This could help $C$. jejuni to adapt and survive within their environment (Vos and Didelot, 2009). However, there are also many bacteria which have much larger rates of recombination such as Helicobacter pylori and Salmonella enterica 
which have recombination rates of $\mathrm{r} / \mathrm{m}=13.6$ and 30.2, respectively (Vos and Didelot, 2009). The higher rate of recombination found in human strains may be due to higher selective pressures within such a host.

A total of 25,091 SNPs were detected outside of recombination regions and were excluded as mutations found in single isolates provide very little information about the shared ancestry between lineages. They are specific to single isolates and could be caused by a number of different factors such as a single mutation (Posada et al., 2002). SNPs found in recombination regions shared between two or more isolates, reveal ancestry and evolution between isolates. A total of 14,825 SNPs were detected within recombination regions which could form the basis for further analysis into what changes have occured.

All C. jejuni strains tested were able to grow at both 37 and $42{ }^{\circ} \mathrm{C}$ as shown previously (Davis and DiRita, 2008; Silva et al., 2011). Overall the optimal growth was at $42^{\circ} \mathrm{C}$ at both high and low oxygen concentrations, irrespective of the source of the isolate. This supports work published previously by Zanetti et al (1996). The optimal growth at $42^{\circ} \mathrm{C}$ with a low oxygen concentration also linked to optimal biofilm formation with the strongest biofilms also seen at this temperature with a low oxygen concentration.

A sub-group of $C$. jejuni strains was chosen to perform biofilm and motility assays based on a number of criteria; i) ST to select representative strains on the Campylobacter phylogenetic tree; ii) presence / absence of motility and biofilm linked genes and iii) the source of the $C$. jejuni strain (Appendix table 8.1). Biofilm production was measured in a variety of different environments and conditions, including under micoaerobic conditions and under normal atmospheric conditions at 37 and $42^{\circ} \mathrm{C}$ to analyse the ability of the $C$. jejuni strains to survive in different conditions that the bacteria could be exposed to in an environmental, industrial or clinical setting (Bolton, 2015; Garénaux et al., 2008; Teh et al., 2014). The strongest biofilms were formed under microaerobic conditions at $42^{\circ} \mathrm{C}$, as shown previously (Davis and DiRita, 2008; Joshua et al., 2006; Pascoe et al., 2015). The strongest biofilms are formed when the strains can grow and proliferate (Davis and DiRita, 2008). All the strains tested were able to form a biofilm with the cut off being set at above $\mathrm{OD}_{600}=0.1$ (Pascoe et al., 2015), although most biofilms formed were weak when compared to those created by bacteria such as Staphylococcus spp. (Archer et al., 2011; Harris et al., 2016). 
The motility of the sub-group of strains was tested later; every $C$. jejuni strain was motile but with a large range of motility. $C$. jejuni strains that were isolated from human sources were the most motile. Interestingly, $C$. jejuni from clinical samples are more pathogenic and identified as hyper-invasive by Fearnley er al (2008) (Fearnley et al., 2008).

This chapter has provided evidence identifying numerous genes in C. jejuni that may provide a survival advantage in the environment and in the host through flagellar motility and transferase genes such as $h d d C$ which relate to an interface between the bacteria and the environment. In the next chapter the role of the host is investigated further by investigating the human and chicken immune responses produced by the 100 C. jejuni strains in a high-throughput in vitro epithelial cell system.

\subsection{Conclusion}

This work adds to the growing number of studies showing the diverse population structure of $C$. jejuni. Irrespective of the source of the isolates, there were differences genomically and phenotypically, which shows that no one strain of Campylobacter can represent how the entire population may act in difference circumstances. A pan-genome approach was applied to a population of $C$. jejuni strains to better understand the spread of virulence genes in the core and accessory genome with GWAS used to identify association between genes and the source animal of a strain. High levels of recombination within the population may also contribute to its survival through rapid evolution via mutations with high recombination rates observed compared to other bacterial species. Scoary was also able to find many genes related to particular sources of $C$. jejuni which could be used for potential markers. To conclude, Campylobacter is highly variable with a diverse population which contains large strain to strain variation and thus there is no one $C$. jejuni isolate that can represent the population. 


\section{Chapter 4: Diverse responses of Campylobacter jejuni strains in human and chicken intestinal epithelial cells}

\subsection{Introduction}

\subsubsection{Campylobacter jejuni pathogenesis in humans and chickens}

C. jejuni is pathogenic to humans and chickens. Studies confirm that $C$. jejuni behaves as a pathogen in chicken hosts although mechanistic understanding is incomplete (Figure 4.1; (Byrne et al., 2007; Humphrey et al., 2014; Jennings et al., 2011; Ruizpalacios et al., 1981; Williams et al., 2013). C. jejuni was first shown to be able to adhere and invade into chicken epithelial cells in vitro by Byrne et al, (2007) with invasion into chicken cells showing an efficiency that was comparable to primary human cells in vitro (Byrne et al., 2007). Later, Jennings et al (2011) discovered a link between $C$. jejuni and the development of vibrionic hepatitis, also known as spotty liver disease which is characterised by the presence of focal lesions that are greyish-white in colour. Chickens with spotty livers contained predominately Campylobacter, thus showing the ability of $C$. jejuni to leave the gastrointestinal tract (Jennings et al., 2011). Further research by Williams et al., (2013) discovered that broiler chickens infected with $C$. jejuni had increased incidence of hock marks and pododermatitis. Thus infection by this bacterium affects the health and welfare of such animals as shown almost 30 years earlier (Williams et al., 2013). Then, Humphrey et al (2014) also showed that $C$. jejuni is not a commensal in broiler chickens and affects bird welfare. Infection led to a large pro-inflammatory innate immune response in some chicken breeds with intestinal inflammation and damage to gut mucosa causing the animals to suffer from diarrhoea. In other broiler types, infection caused much less damage to the gut mucosa and the animals remained largely well Humphrey et al (2014). Campylobacter jejuni strains also differ in virulence in chickens.

\subsubsection{Role of epithelial cells in Campylobacter pathogenesis}

$C$. jejuni has been shown to adhere efficiently to and enter chicken intestinal epithelial cells. in vitro and in vivo mice models confirm consistent roles for pathogen recognition by the host through TLRs-2, $-4,-5$ and -9 following Campylobacter infection (Friis et al., 2009; Hara et al., 2012; Rathinam et al., 2009). However, a recent study suggests that there may well be differential activation between human and chicken receptors adding to the complexity of responses (De Zoete et al., 2010). 

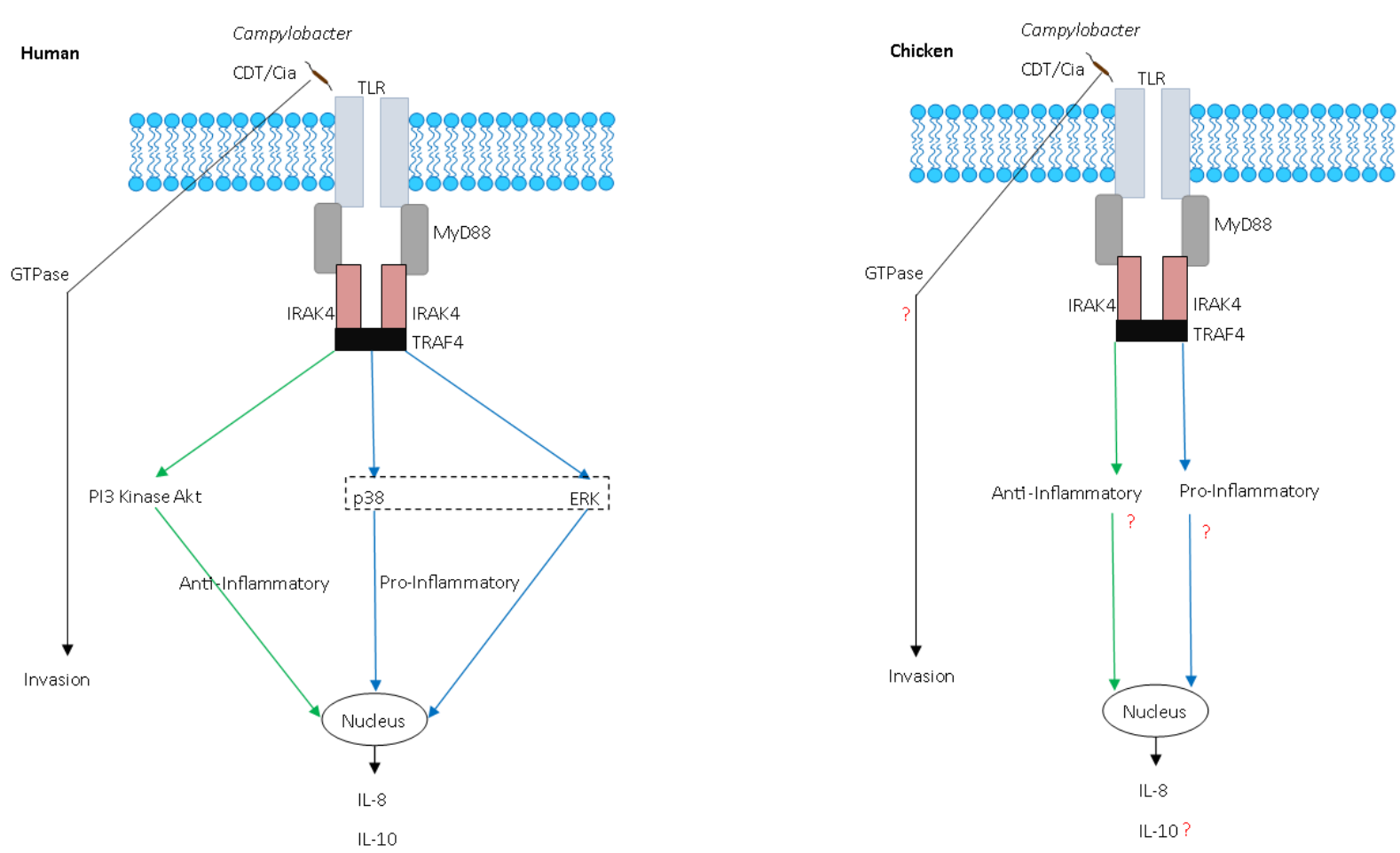

Figure 4.1. Interaction of Campylobacter with host epithelial cells in human and chicken derived cells

\subsubsection{Campylobacter induced signalling pathways in epithelial cells}

Early host cell responses to Campylobacter are better understood in human than with chicken intestinal epithelial cells (Figure 4.1). Thus, Campylobacter flagellin stimulates TLR5 found on the apical surface of intestinal epithelial cells to initiate signalling for CXCL8, the gene encoding for IL-8 in humans (Borrmann et al., 2007) and CXCLil and CXCLi2 chickens (Larson et al., 2008).

In human intestinal epithelial cells, Campylobacter activates signalling pathways such as phosphoinositide 3-kinase/protein kinase B/Akt (PI3K/Akt) and the mitogen activated protein kinase (MAPK) pathways resulting in the induction of IL-8 and IL-10. In addition, adhesion and invasion by $C$. jejuni, along with its secreted toxin CDT, are thought to be the main factors driving IL-8 production (Li et al., 2011). Interestingly, inhibition of the PI3K/Akt signalling pathway in human epithelial cells infected with $C$. jejuni resulted in a reduction of IL-10 and an increase in IL-8 production which may help $C$. jejuni survive intracellularly suggesting that pro and anti-inflammatory pathways exist in human intestinal cells (Li et al., 2011). In contrast, there has been a lack of work regarding the action of Campylobacter in chicken epithelial cells lines compared to the vast research into responses in human cell lines (Hickey et al., 1999, 2000; Jin et al., 2003; Jones et al., 2003). In addition, in the chicken macrophage cell 
line (HD11) there is evidence that there is a robust inflammatory response to Campylobacter spp. (Smith et al., 2005). Therefore, there is increasing evidence that Campylobacter is not merely a commensal in chickens and causes an immune response as discussed in previously (Humphrey et al., 2014). Further understanding the response of chicken cells to Campylobacter forms the basis for the work in this chapter.

\subsubsection{Aims}

Using the $100 C$. jejuni strains characterised in chapter 3 the inflammatory (cytokine), adhesion, invasion, toxicity and signalling responses induced in HT-29 (human) and 8E11 (chicken) intestinal epithelial cells were investigated to:

- Characterise a novel commercially available chicken epithelial cell line, 8E11

- Determine the inflammatory responses of chicken epithelial cells to $C$. jejuni strains

- Investigate how the presence/absence of known virulence genes in C. jejuni affect the inflammatory response

- Investigate the signalling pathways responsible for gene expression and $C$. jejuni invasion responses

- Associate Campylobacter virulence genes to in vitro cell responses by Scroary GWAS pipeline. 


\subsection{Materials and Methods}

\subsubsection{Bacterial isolates}

Bacteria were grown on either Oxoid CBA (Thermo-scientific) or on mCCDA (Oxoid) plates and incubated at $42^{\circ} \mathrm{C}$ under microaerobic conditions using CampyGen ${ }^{\mathrm{TM}} 3.5 \mathrm{~L}$ sachets (Thermo-scientific). C. jejuni strains were cultured under microaerobic conditions $\left(5 \% \mathrm{O}_{2}, 10 \% \mathrm{CO}_{2}, 85 \% \mathrm{~N}_{2}\right)$. A single colony was inoculated into MullerHinton broth (Oxoid) and incubated at $37^{\circ} \mathrm{C}$ for 24 hours. All isolates used were subcultured from a stock stored at $-80^{\circ} \mathrm{C}$. All $C$. jejuni strains used in this study can be seen in Appendix, Table 8.2.

\subsubsection{Culture of human and chicken intestinal epithelial cells}

The culture of both human and chicken lines was performed as described in section 2.2.3

\subsubsection{Infection studies}

Cell monolayers were infected with $50 \mu \mathrm{l}$ of bacterial suspension, containing approximately $3.5 \times 10^{5} \mathrm{CFU}$ per well. The infected monolayers were incubated for 4,6 and 24 hours at $37^{\circ} \mathrm{C}$ in a $5 \% \mathrm{CO}_{2}$ atmosphere to allow the bacteria to adhere and invade the host cells. Uninfected cells and ones treated with PMA were included in the experiments as a negative and positive control.

\subsubsection{Human cytokine antibody array assay}

The human cytokine antibody array membrane with 42 targets (Appendix Table 8.10) (Abcam, Cambridge, UK) was used to measure and detect 42 human cytokines from human cell lines infected or uninfected with two typed $C$. jejuni strains, 11168 and M1 (Table 2.2). Assays were performed according to the manufacturer's instructions; briefly, the membranes were treated with $2 \mathrm{ml}$ of the blocking buffer provided. Then, $1 \mathrm{ml}$ of the culture supernatants from infection studies and from an uninfected control sample were added for 2 hours at room temperature. Membranes were washed three times using the wash buffers I and II, provided. Then, $1 \mathrm{ml}$ of the biotin-conjugated anticytokine antibody conjugate was added to each membrane and incubated overnight at $4^{\circ} \mathrm{C}$. Membranes were washed again before $2 \mathrm{ml}$ of HRP-conjugated Streptavidin was added to each well and incubated at room temperature for 2 hours. The membranes were washed again prior to chemiluminescence detection. The membranes were treated with $500 \mu \mathrm{l}$ of the detection buffers provided and incubated for 2 minutes at room 
temperature. They were then imaged using BioRad ChemiDoc XRS+ (BioRad, UK). The images were analysed using ImageJ to plot mean pixel density.

\subsubsection{Enzyme linked immunosorbant assay (ELISA)}

The ELISAs were performed as described in section 2.2.5.

\subsubsection{RNA Isolation}

Total RNA was isolated from HT-29 human or 8E11 chicken intestinal epithelial as described in section 2.2.6.

\subsubsection{Quantitative PCR of RNA transcripts}

Quantitative PCR was performed using SYBR green as described in section 2.2.7. Primer efficiency was measured using total RNA from epithelial cells infected with a reference $C$. jejuni isolate (NCTC11168) and a dilution series up to $1 / 10,000$. Target sequences were identified from the NCBI database and then the coding sequence (CDS) was used to generate forward and reverse primers using Primer3, selecting for amplicon sizes of 50-150 bases.

\subsubsection{PCR Conditions}

The BioRad iCycler was run using conditions described in section 2.2.9.

\subsubsection{Invasion Assay and Adhesion}

The invasion and adhesion assay was performed as described in section 2.2.11.

\subsubsection{Cell Viability}

The cell viability assay was performed as described in section 2.2.12.

\subsubsection{Signal Inhibition Assays}

The signal inhibition assays were performed as described in section 2.2.14. The inhibitors and their working concentrations used are summarsed in Table 4.1.

\subsubsection{GWAS using Scoary}

Scoary microbial pan-GWAS (Brynildsrud et al., 2016) was performed as described in section 2.3.3. Scoary was used to identify genes that were related to the different phenotypes identified throughout the chapter (including IL-8, IL-10 expression, and invasion response). 
Table 4.1. List of inhibitors and their respective pathways used during this study.

\begin{tabular}{|c|c|c|}
\hline Inhibitor & Pathway & Reference \\
\hline Dynasore $(80 \mu \mathrm{M})$ & $\begin{array}{l}\text { Dynamin - Endocytosis, } \\
\text { Dynamin GTPase activity }\end{array}$ & (Macia et al., 2006) \\
\hline Filipin $(1 \mu \mathrm{g} / \mathrm{ml})$ & $\begin{array}{l}\text { Lipid raft Caveolin pathway } \\
\text { Endocytosis }\end{array}$ & (Bonneau et al., 2010) \\
\hline Genistein $(1 \mu \mathrm{g} / \mathrm{ml})$ & $\begin{array}{l}\text { Caveolin Endocytosis, tyrosine } \\
\text { kinase inhibitor }\end{array}$ & (Akiyama et al., 1987) \\
\hline Chlorpromazine $(5 \mu \mathrm{g} / \mathrm{ml})$ & $\begin{array}{l}\text { Clathrin Endocytosis, clathrin } \\
\text { misassembly }\end{array}$ & (Lee et al., 2007) \\
\hline LY294002 $(10 \mu \mathrm{M})$ & P-I3 Kinase & (Chaussade et al., 2007) \\
\hline $\begin{array}{l}\text { InSolution }^{\mathrm{TM}} \text { AKT Inhibitor } \mathrm{V} \text {, } \\
\text { Triciribine }(10 \mu \mathrm{M})\end{array}$ & AKT & (Karst et al., 2006) \\
\hline Methyl $\beta$ cyclodextrin $(5 \mu \mathrm{g} / \mathrm{ml})$ & $\begin{array}{l}\text { Lipid raft disruption by } \\
\text { cholesterol extraction }\end{array}$ & (Stella and He, 2008) \\
\hline Cytochalasin D $(5 \mu \mathrm{g} / \mathrm{ml})$ & Actin polymerisation & (May et al., 1998) \\
\hline PD98059 $(10 \mu \mathrm{g} / \mathrm{ml})$ & ERK/MEK & (Alessi et al., 1995) \\
\hline
\end{tabular}

\subsubsection{Statistics}

The non-parametric Krustal-Wallis test, with a post hoc Dunns test for multiple comparisons was used. Correlations were assessed using linear regression of log transformed data with a $\mathrm{p}$ value related to the slope. Significance differences were accepted if $\mathrm{p} \leq 0.05$. Graphpad Prism 6.0 (San Diego, USA) was used to analyse and assess differences between treatment groups. 


\subsection{Results}

\subsubsection{C. jejuni strains used in this study}

To investigate the diversity of human and chicken epithelial cell innate immune responses, the 100 strains of $C$. jejuni used in chapter 3 were investigated (Figure 3.2, Table 2.2). Isolates were from a variety of STs, and the major clonal complexes CC-45 and CC-21 (Figure 3.2). In addition, strains were selected based on the source of the isolate and included, human, chicken, cattle and from wild-bird (Figure 3.2).

\subsubsection{Identification of potential immune markers from cytokine panel array assays}

A human cytokine antibody array assay was performed using the two reference strains for C. jejuni; 11168 and M1 (Figure 4.2a). Samples taken from uninfected HT-29 cells showed very little to no expression of all cytokines measured, with the positive controls in the top left corner and bottom right corner being the only signals present on the membrane (Figure 4.2a). The major cytokine induced in response to 11168 and M1 was IL-8 (CXCL8). Other cytokines also found to be expressed included IP-10 (CXCL10), MIP-1 $\delta$ (CCL15), RANTES (CCL5), TNF RI (TNFRSF1A) and TIMP-2 (Figure 4.2). A list of all cytokines measured during this assay is shown in appendix table 8.10. 

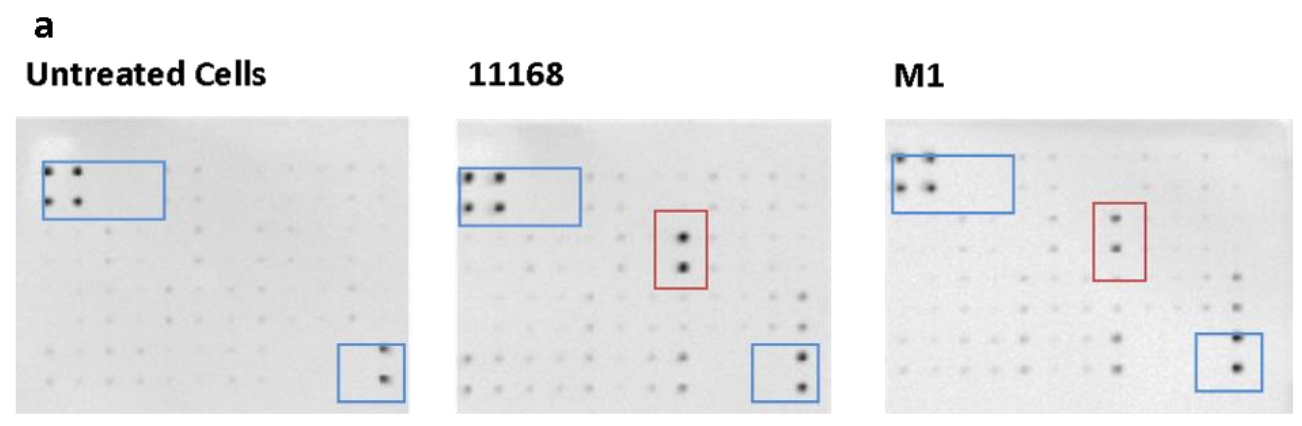

\section{b}

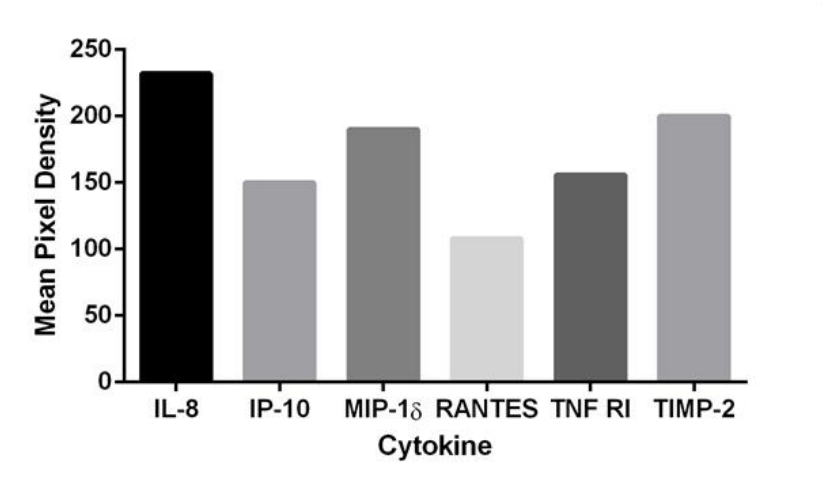

Figure 4.2. Human cytokine array assay performed on uninfected cells, cell infected by 11168 and cells infected by M1. Red box indicates the IL-8 cytokine expression in 11168 and M1; blue box indicates the control wells (a), with markers with the overall largest mean pixel density in the infected samples plotted (b).

\subsubsection{IL-8 secretion from human epithelial cells}

The cytokine membrane identified IL-8 as an important early gene induced by $C$. jejuni. To investigate the epithelial response further, the 100 C. jejuni strains from chapter 3 were used to infect HT-29, human intestinal epithelial cells. IL-8 secretion from HT-29 cells increased over the 24 hours of co-culture with the $C$. jejuni strains (Figure 4.3). Uninfected cells produced little or no IL-8 whereas the positive control (PMA) treated wells had significantly increased output of this cytokine. Comparison between time points demonstrated highly significant differences between IL-8 production at 4-24 hours (4 vs 24 hours, $\mathrm{P}<0.0001$ ). Large variations in IL-8 production were observed in response to the different $C$. jejuni strains with the highest and lowest IL-8 production being isolate 79 with an average IL- 8 secretion of $2234.5 \mathrm{pg} / \mathrm{ml}$ and isolate 56 with an average IL-8 secretion of $40.75 \mathrm{pg} / \mathrm{ml}$ resulting in a 50 -fold difference in production.

Comparison of IL-8 secretion from HT-29 cells in response to $C$. jejuni strains from different sources over the same time course (Figure 4.3) was also assessed. After 4 hours (Figure $4.3 \mathrm{~b}$ ) of infection very little IL-8 was produced in response to all strains 
with no significant differences being shown between them (Fig 4.3b). After 6 hours of infection, IL-8 production had increased compared to controls (Figure 4.3c) and to secretion after 4 hours; with strains isolated from wild birds beginning to emerge with the highest overall IL-8 secretion, although no significant difference was detected at these time points.

After 24 hours of infection, very large differences were observed in IL-8 secretion compared to control (Figure 4.3d). In addition, IL-8 secretion in response to isolates from wild birds showed the highest levels with a significant difference when compared to the strains isolated from humans $(\mathrm{P}>0.001)$, chickens $(\mathrm{P}>0.01)$ and cattle $(\mathrm{P}>0.0001)$. Large differences were also observed between strains from the same source, such as in strains from chickens where differences in IL-8 secretion as large as $2200 \mathrm{pg} / \mathrm{ml}$ (Figure 4.3d) were observed. Distributions were assessed for 'normality' by the D'agostinoPearson test which confirmed that the results obtained were not normally distributed.

When these results were grouped by $C$, jejuni clonal complex (Figure 4.4) strains from ST-45 show a higher overall IL-8 secretion (by approximately $250 \mathrm{pg} / \mathrm{ml}$ ) than ones from ST-21 (Figure 4.4). However, there was no significant difference $(\mathrm{P}>0.05)$ between the two clonal complexes or when these ST were then compared to isolates from unique and other clonal complexes (Figure 4.4). 
a

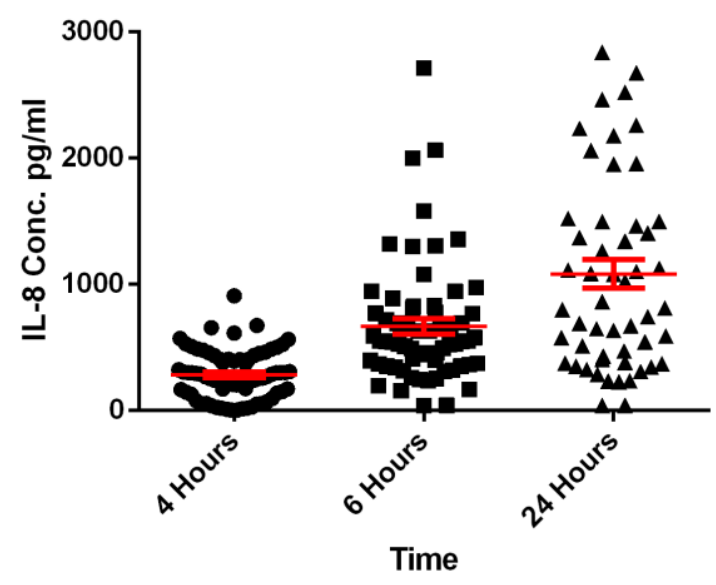

C

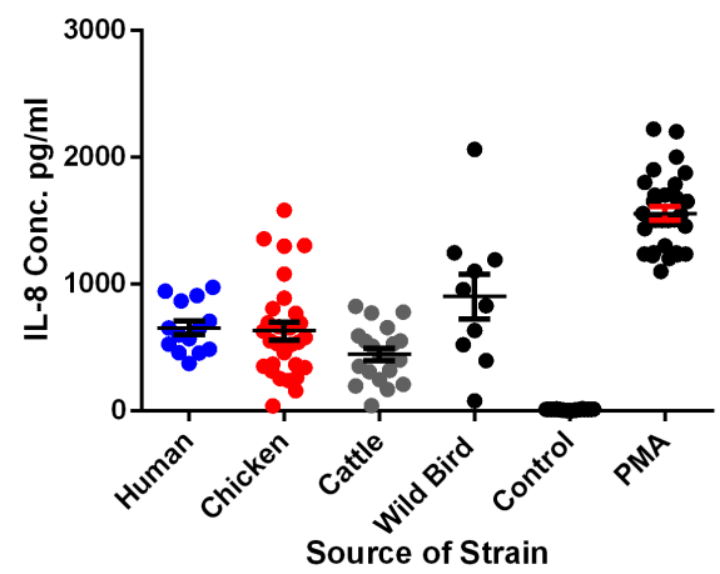

b

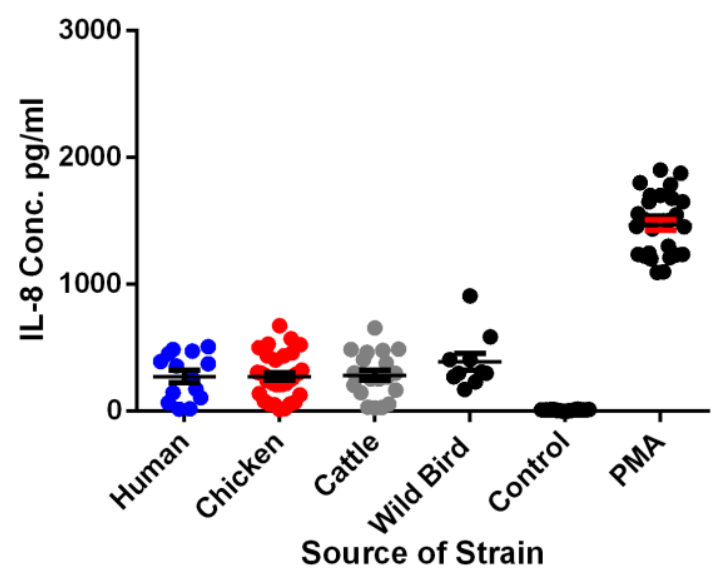

d

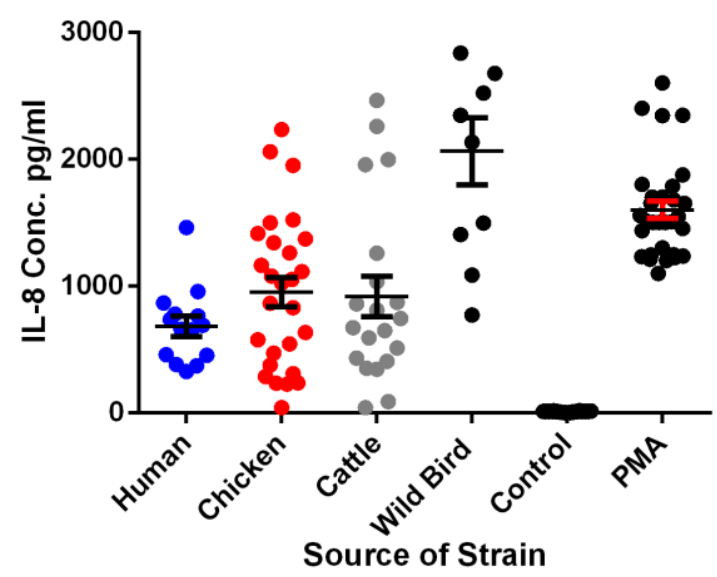

Figure 4.3 Mean IL-8 secretion induced by $C$. jejuni at (a) over all time points measured, (b) $4 \mathrm{~h}$ of infection, (c) $6 \mathrm{~h}$ of infection and (d) $24 \mathrm{~h}$ of infection categorised by source of Campylobacter strain and d) Mean IL-8 secretion induced by C. jejuni at 24h, categorised by source of Campylobacter strain. Results are also expressed as mean +/$\mathrm{SD}$ of all strains measured. Differences were considered significant if $\mathrm{p} \leq 0.05$. 

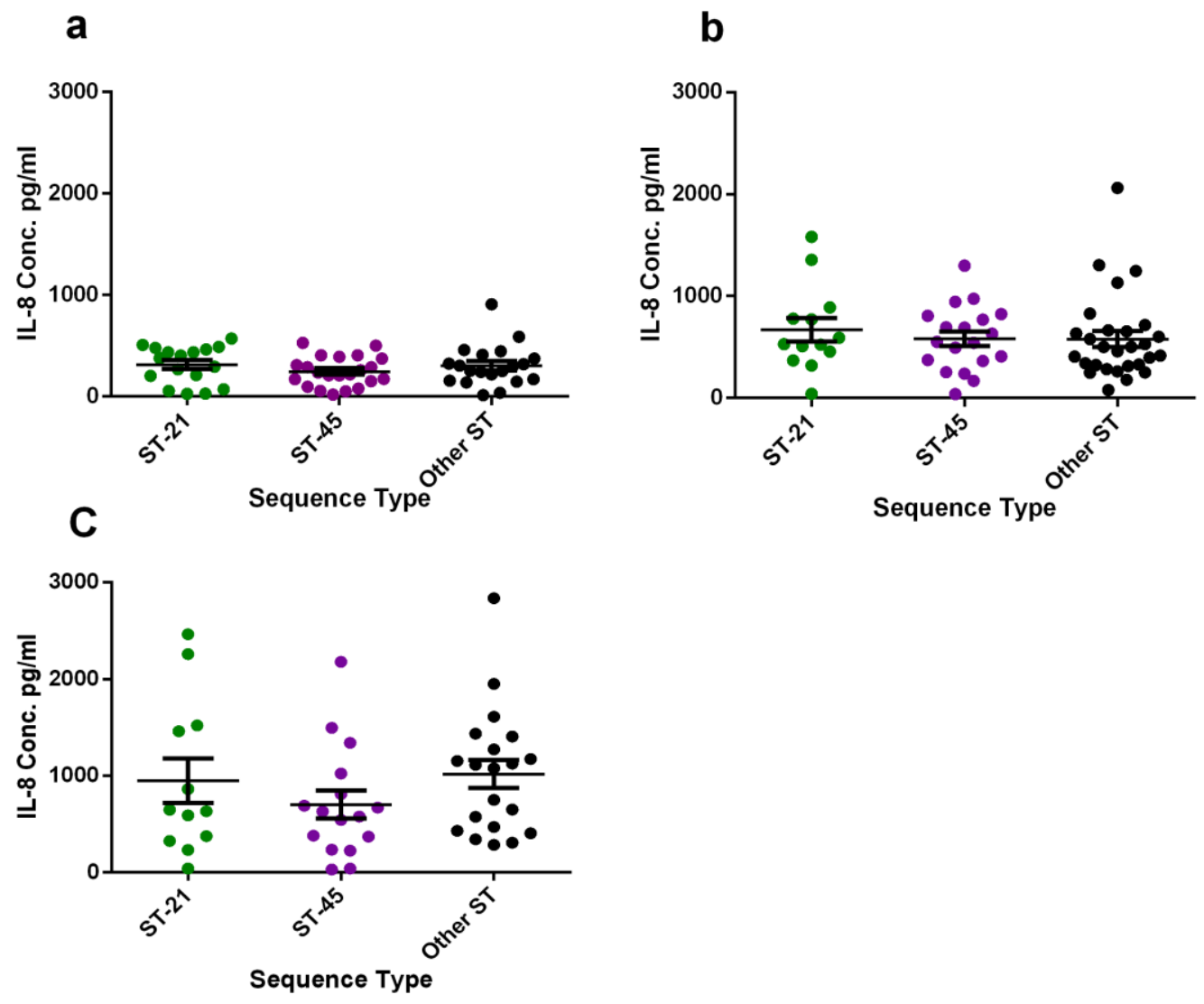

Figure 4.4. Mean IL-8 secretion induced by $C$. jejuni at (a) $4 \mathrm{~h}$ of infection (b) $6 \mathrm{~h}$ of infection (c) $24 \mathrm{~h}$ of infection categorised by sequence type the of Campylobacter strain. Results are also expressed as mean $+/-$ SD of all strains measured. Differences were considered significant if $\mathrm{p} \leq 0.05$.

\subsubsection{Confirmation of the species of the chicken cell line using MHC expression}

MHC Class 1 ( $\beta$-macroglobulin) expression was used to measure the cell lines capability to present foreign molecules and to speciate the cells. MHC class 1 was investigated after 24 hours of co-culture of the cell lines with the $C$. jejuni isolates. Overall expression was greater in human cells than in chicken ones with a significant difference $(p=\leq 0.001)$ between the two (Figure 4.5$)$.

Expression in the HT-29 cells (Figure 4.5b) displayed large variations between isolates from different sources, with those from chickens showing the largest expression and significant differences were found between human and chicken isolates $(p \leq 0.001)$ and human and cattle isolates $(\mathrm{p} \leq 0.01)$. The HT-29 cells displayed very similar expression levels of MHC with all the isolates from human sources. Similar expression patterns 
were observed within expression from the 8E11 chicken cells (figure 4.5c). However, the highest level of expression was seen in isolates from cattle. Significant differences between the human isolates and the chicken isolates $(p \leq 0.01)$ and cattle isolates $(p \leq 0.001)$ were seen. The results also confirm the 'chicken' nature of the 8E11 cells.

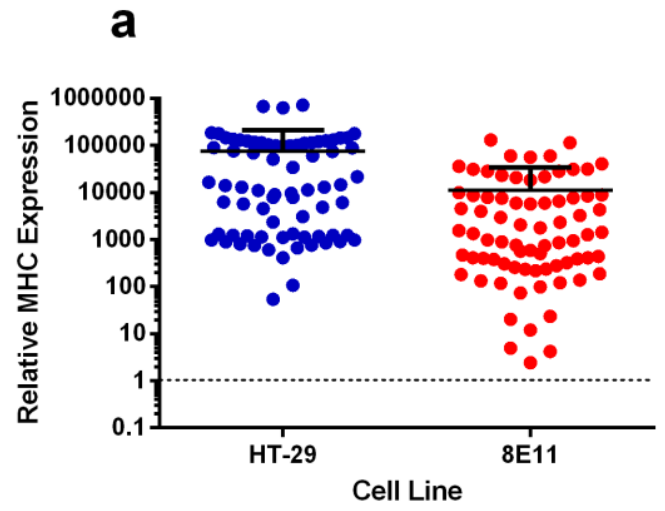

b
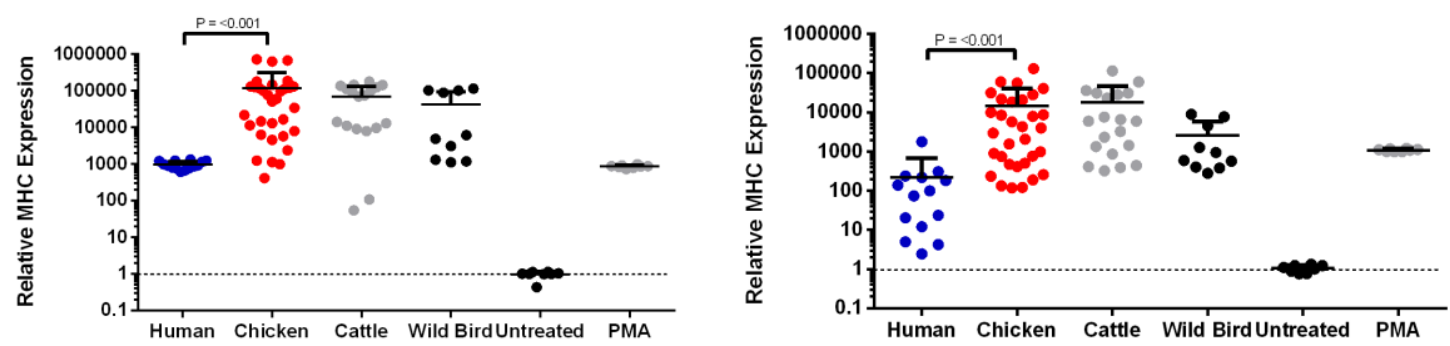

d

e
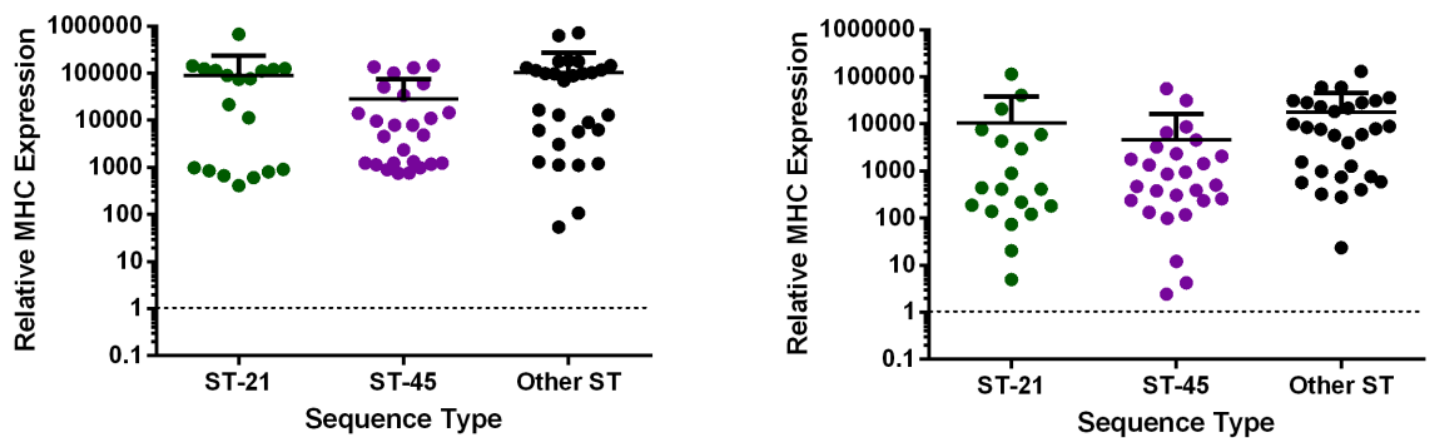

Figure 4.5. Expression of MHC induced by C.jejuni after $24 \mathrm{~h}$ of infection on (a) HT-29 and 8E11 cell lines; with (b,c) the breakdown of responses by source in HT-29 (b)/8E11(c) cells and (d,e) by sequence type of Campylobacter in HT-29(d) and 8E11(e) cells. Results are also expressed as mean +/- SD of all strains measured. Differences were considered significant if $\mathrm{p} \leq 0.05$. 


\subsubsection{C. jejuni strains produce a large variation in inflammatory cytokine responses}

Inflammatory phenotype was investigated by infecting human and chicken epithelial cells with the $C$. jejuni strain collection $(\mathrm{n}=100)$. IL-8 or CXCLi1 and CXCLi2 expression in these cells showed dramatic changes compared to uninfected controls with up to 100,000-fold increases in both human and chicken cells (Figure 4.6a). Despite the large variation, human IL-8 and chicken CXCLi2 expression were significantly increased ( $\mathrm{p} \leq 0.01$ and $\mathrm{p} \leq 0.01$ respectively) compared to CXCLi1. There was no difference between IL-8 and CXCLi2 expression. The reference strains NCTC11168 and M1 produced IL-8 and CXCLi1 responses similar to the average for the whole $C$. jejuni study population. The average $C$. jejuni induced CXCLi2 response was similar to that of the M1 but the 11168 strain induced response was 11 -fold higher $(p \leq 0.01)$. The positive control, PMA, produced similar high levels of expression with both cell lines and with each cytokine measured. There were no differences seen when comparing the two most common sequence types of $C$. jejuni; ST-21 and ST-45.

The anti-inflammatory cytokine IL-10 also displayed an infection-induced increase in expression and variability compared to uninfected controls $(\mathrm{p} \leq 0.001)$ in both HT-29 and 8E11 cells. In addition, IL-10 expression was significantly increased $(\mathrm{p}<0.001)$ in 8E11 cells compared to that in HT-29 cells (Figure 4.6b). The reference strains NCTC11168 and M1 produced IL-10 responses similar to the average for the whole C. jejuni study population. 

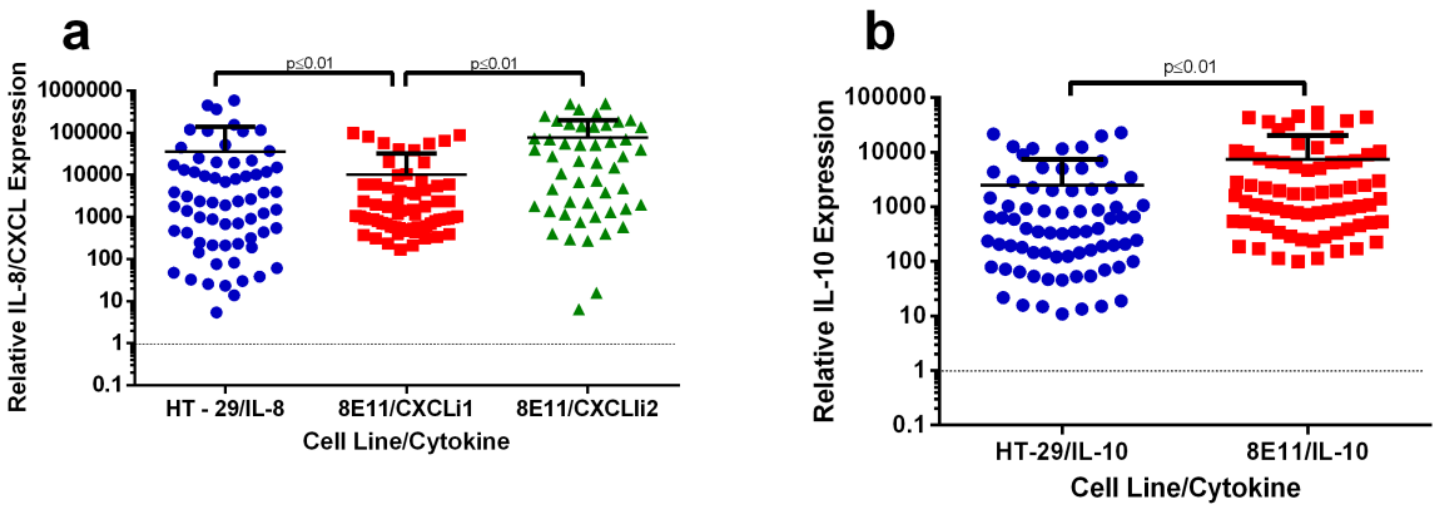

Figure 4.6 Expression of pro- and anti- inflammatory cytokines and chemokines in HT29 human and 8E11 chicken epithelial cells after infection with $C$. jejuni for 24 hours before isolation of total RNA and quantification of specific mRNA by qPCR. (a) Expression of IL-8/CXCLi1/CXCLi2 in both HT-29 and 8E11 cell lines (b) Expression of IL-10 in HT-29 and 8E11 cell lines. Results are also expressed as mean +/- SD of all strains measured. Differences were considered significant if $\mathrm{p} \leq 0.05$.

\subsubsection{Inhibition of $C$. jejuni cytokine response}

Following on from the cytokine expression work, eight strains of $C$. jejuni that produced the strongest cytokine responses were chosen to investigate whether they had an effect on common signalling pathways (PI 3-kinase/Akt and ERK) in human and chicken epithelial cells (Figure 4.7 a, b and c). Inhibition of signalling pathways showed that IL8 expression in HT-29 cells and CXCLi1 and CXCLi2 in 8E11 cells were all ERKdependent ( $\mathrm{p} \leq 0.001, \mathrm{p} \leq 0.01$ and $\mathrm{p} \leq 0.05$ respectively). Furthermore, PI 3-kinase and Akt pathways did not appear to be required for IL-8 or CXCLi1/2 production. In contrast, IL-10 expression in human and chicken cells was dependent on PI 3-Kinase and its downstream target Akt but was independent of ERK (Figure 4.7d and e). These results confirm that similar signalling pathways are responsible for IL-8 and IL-10 expression in human and chicken epithelial cells. 
a

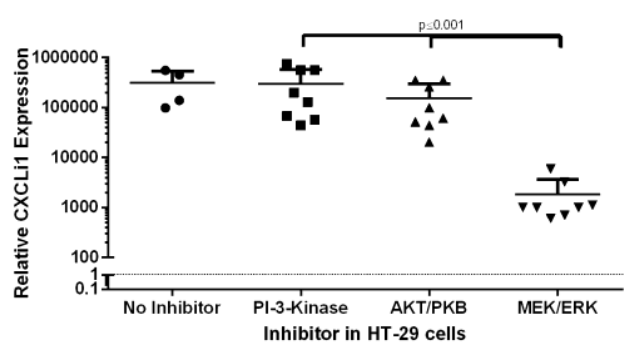

C

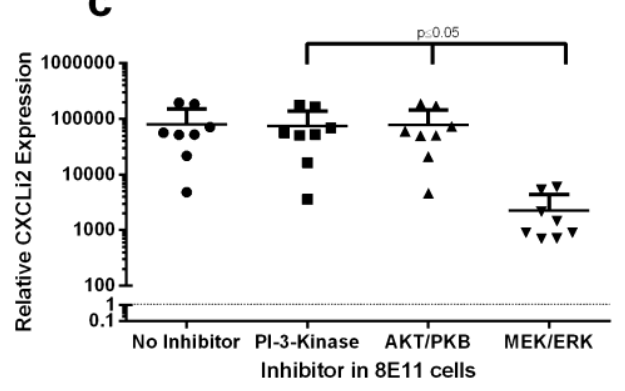

e

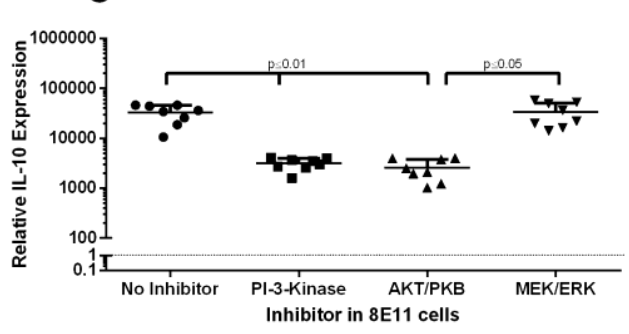

b

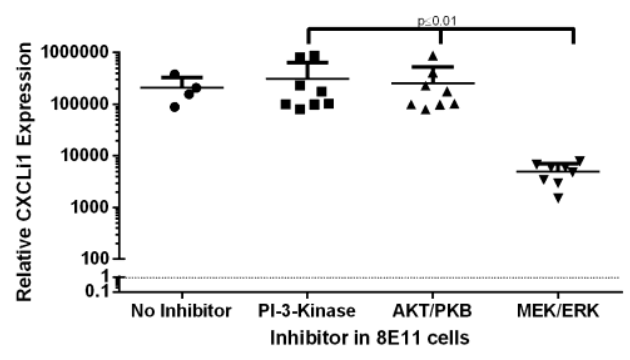

d

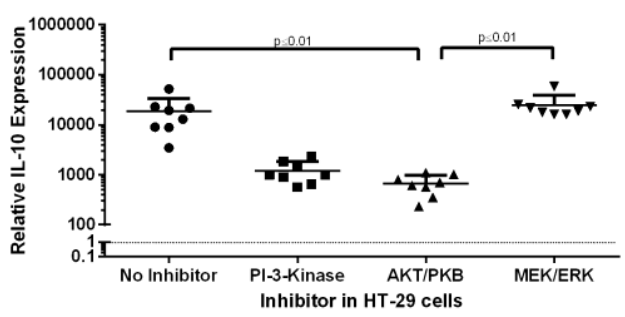

Figure 4.7. Prior to infection with $C$. jejuni, cells were incubated with signalling inhibitors for up to 30 minutes. Then specific mRNA for IL-8 / CXCLi1 / CXCLi2 (a, b, c) and IL-10 (d, e) were measured. Each dot represents three biological replicates in one strain. Results are also expressed as mean +/- SD of all strains measured. Differences were considered significant if $\mathrm{p} \leq 0.05$.

\subsubsection{The $c d t A$ gene has an important role in $C$. jejuni-induced epithelial cell toxicity}

The influence of invasion by all $C$. jejuni strains $(\mathrm{n}=100)$ on epithelial cell viability and toxicity responses was investigated (Figure 4.8). The toxicity of $C$. jejuni on both human and chicken cells showed unique profiles for each strain tested. Epithelial cells infected with any $C$. jejuni strain showed increased toxicity compared to untreated cells but only a few $C$. jejuni strains induced high toxicity responses (over $50 \%$ reduction in viability, Figure 4.8a). There were no differences between sources and sequence types. $c d t A$-positive strains were significantly more toxic than -negative ones in both human (Figure 4.8b, $p=\leq 0.0001$ ) and chicken epithelial cells (Figure 4.8c, $p=\leq 0.001$ ). 

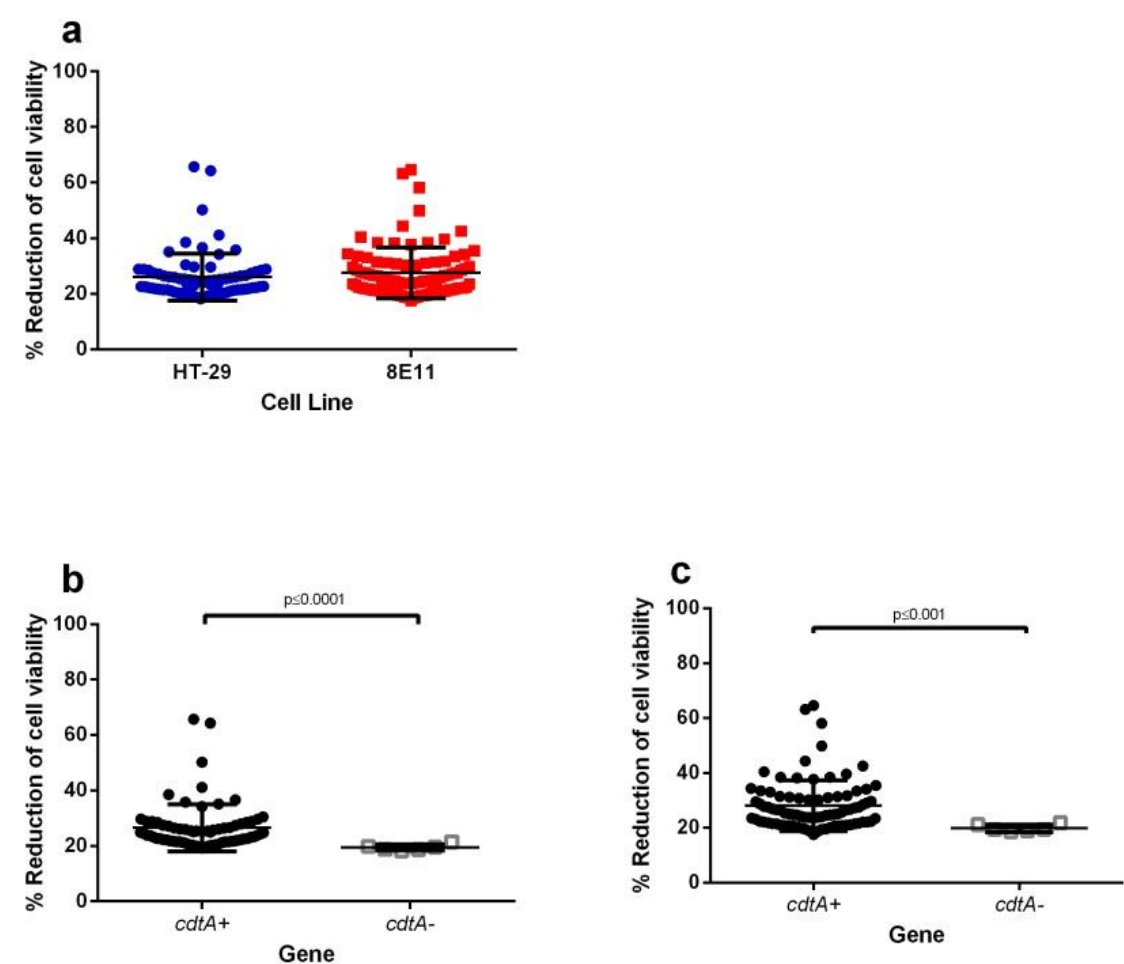

Figure 4.8. Reduction of cell viability after infection with $C$. jejuni. HT-29 and 8E11 cells were infected with $C$. jejuni for 24 hours before isolation of cell supernatants for alamar blue viability assay. (a) Toxicity responses in HT-29 and 8E11 epithelial cells. (b and c) Toxicity responses organised by the presence and absence of the $c d t A$ gene. Each dot represents three biological replicates in one strain. Results are also expressed as mean $+/-$ SD of all strains measured. Differences were considered significant if $\mathrm{p} \leq 0.05$.

\subsubsection{Measurement of $C$. jejuni adhesion and invasion in human and chicken epithelial cells}

The ability of high and low IL-8 (or CXCLi1/2)-inducing $C$. jejuni strains (n=35) to adhere to and invade human and chicken epithelial cells was investigated. No significant difference $(\mathrm{P}>0.05)$ was detected in adherence to human and chicken epithelial cells (Figure 4.9a) although adhesion levels to chicken cells had a wider distribution. Gentamicin protection assays with both cell lines showed that all strains tested were able to invade cell lines (Figure 4.9b), ranging from 1-3\% of the starting inoculum. While each strain produced a unique invasion response no significant difference $(\mathrm{P}>0.05)$ in the invasion was observed between human and chicken cells. Inhibition of epithelial cell signalling pathways following invasion with 'high' invasive strains $(\mathrm{n}=8)$ confirmed the role of ERK in $C$. jejuni invasion of human (Figure 4.9c) and chicken (Figure 4.9d) epithelial cells $(\mathrm{p}=\leq 0.001$ in both cases). These results 
confirm the unique invasion properties of individual $C$. jejuni strains despite all requiring ERK for a full invasion response.

a

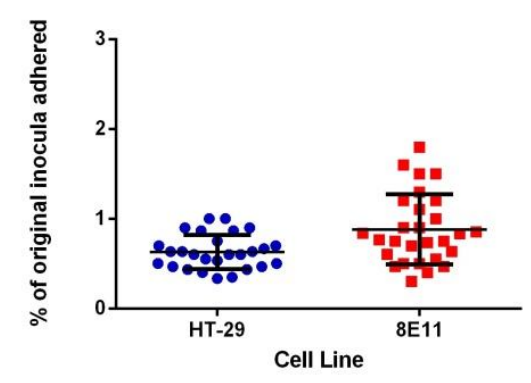

C

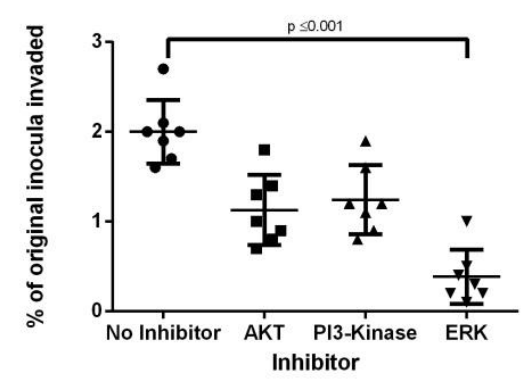

b

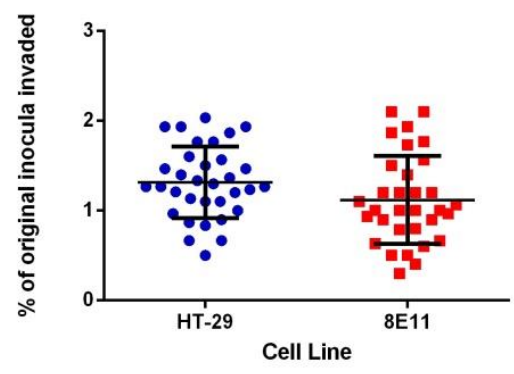

d

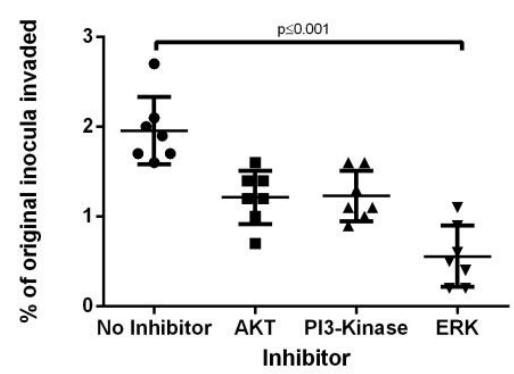

Figure 4.9 HT-29 and 8E11 cells were infected with $C$. jejuni for 6 hours before isolation and quantification of adherent and invasive C. jejuni. (a) Adhesion of C. jejuni strains to HT-29 and 8E11 cells. (b) Invasion of $C$. jejuni into HT-29 and 8E11 cells. (c,d) Treatment of HT-29 and 8E11 cells with signalling inhibitors for 30 minutes prior to invasion assays. Each dot represents three biological replicates in one strain. Results are also expressed as mean $+/-$ SD of all strains measured. Differences were considered significant if $\mathrm{p} \leq 0.05$. 

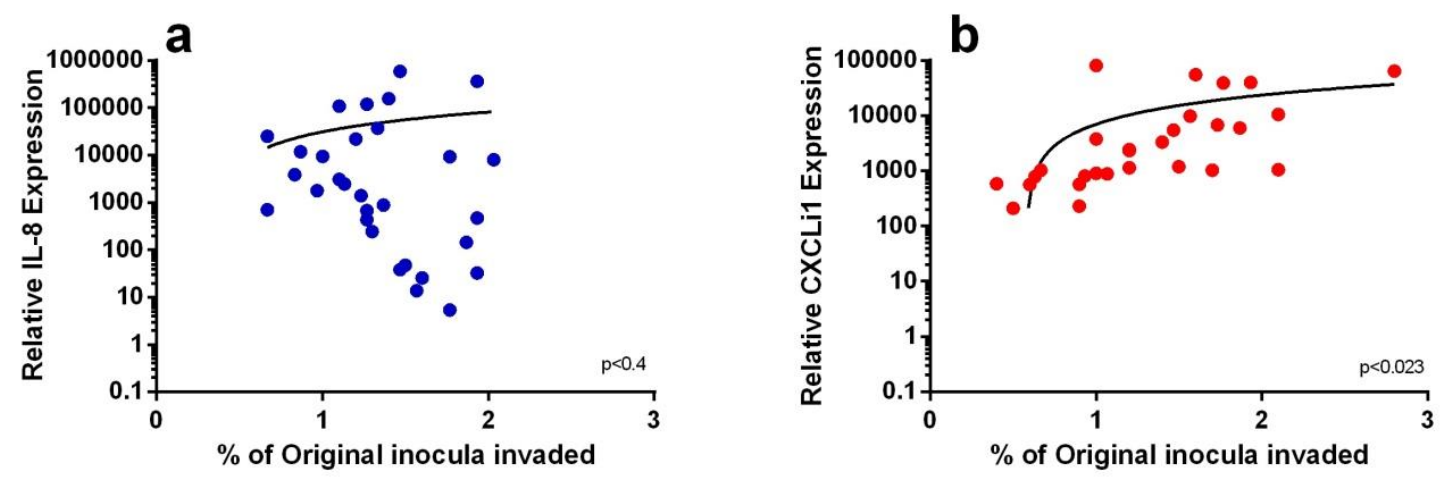

C
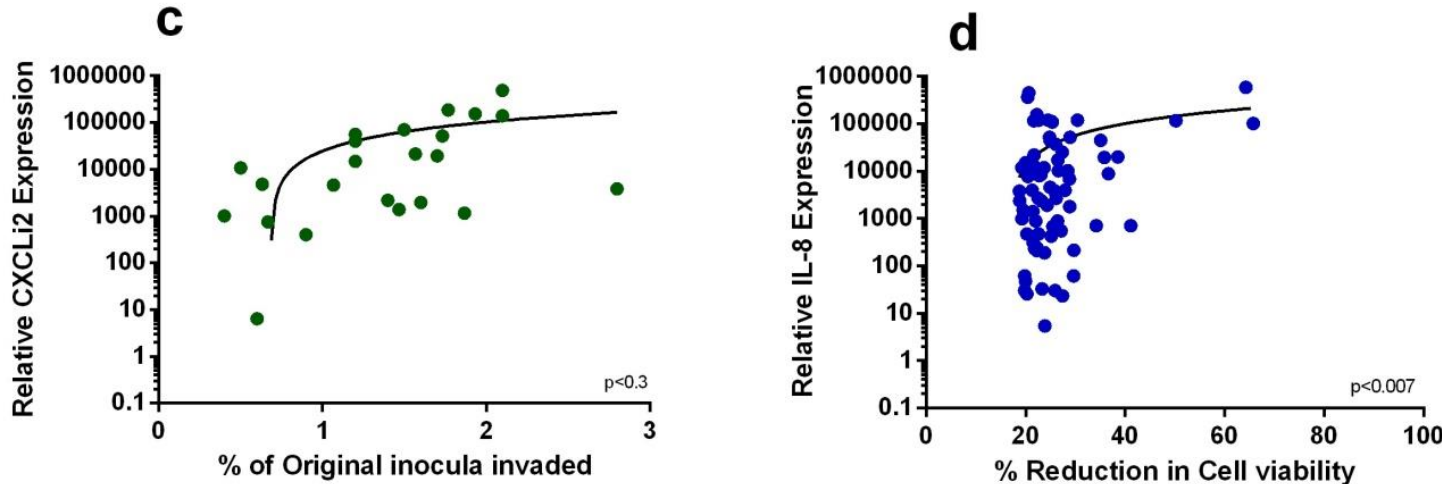

e
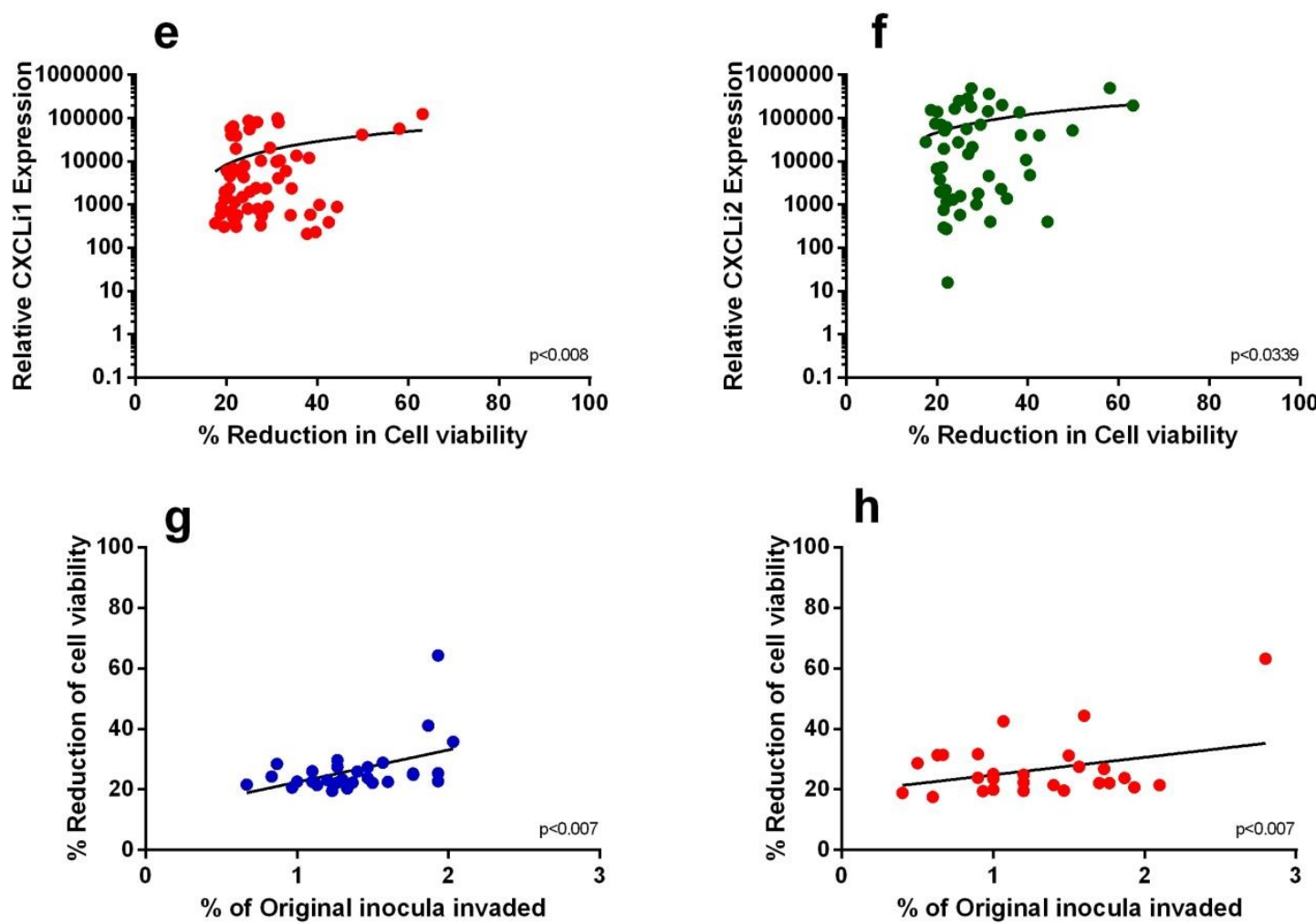

Figure 4.10. Relationships between $C$.jejuni-induced cellular invasion, toxicity and cytokine production; phenotypic data was subjected to regression analysis. (a) IL-8 (b) CXCLi1and (c) CXCLi2 correlations with $C$. jejuni invasion. (d) IL-8, (e) CXCLi1, (f) CXCLi2 correlations with toxicity. (g) HT29 cell viability correlation with invasion (h) 8 E11 cell viability correlation with invasion. 


\subsection{9 $C$. jejuni induced IL-8 production, toxicity and invasion of epithelial cells are more closely correlated in chicken than human cells}

Given that both IL-8 production and invasion were ERK-dependent across the collection of $C$. jejuni strains, correlations between the measured phenotypes of IL-8 production, invasion and toxicity were performed. IL-8 expression and invasion were not correlated (Figure 4.10a, p<0.4) whereas both CXCLi1 (Figure 4.10b, p<0.023) and CXCLi2 (Figure 4.11c, p<0.03) expression showed strong positive correlations with invasion. IL-8 ( $<<0.007)$, CXCLi1 $(\mathrm{p}<0.0082)$ and CXCLi2 $(\mathrm{p}<0.0339)$ all positively correlated with cell toxicity (Figures 4.10d-f). Finally, invasion and toxicity were strongly correlated in human cells $(\mathrm{p}<0.0078)$ but did not reach significance in chicken ones (Figure $4.10 \mathrm{~g}$ and $\mathrm{h}$ ). These correlations confirm the close interrelationship between $C$. jejuni-induced IL-8 expression, toxicity and cellular invasion.

\subsubsection{Endocytosis of $C$. jejuni is dynamin- and clathrin-dependent in both human and chicken epithelial cells}

Having confirmed the importance of ERK in downstream signalling for invasion, upstream pathways at the cell surface important for endocytosis were analysed (Figure 4.11). Both Methyl- $\beta$-cyclodextrin and cytochalasin D completely abrogated $C$. jejuni invasion into both cell lines providing more evidence for the role of lipid rafts and the actin cytoskeleton respectively. Pre-treatment of epithelial cells with a dynamin inhibitor (Dynasore) and a clathrin inhibitor (chlorpromazine) reduced significantly $C$. jejuni invasion in human (Figure 4.11a) and chicken (Figure 4.11b) cells compared to the 'no' inhibitor control. This is confirmed by concomitant reduction in cellular toxicity in the relevant cultures (Figure 4.12). In addition, caveolin-dependent endocytosis was tested using filipin and genistein with no consistent effect observed over triplicate experiments. These results confirm the importance of clathrin and dynamin in $C$. jejuni invasion. The inhibitors Methyl- $\beta$-cyclodextrin and cytochalasin D were able to completely block invasion of $C$. jejuni (Figure 4.11). However, they were also very toxic to both cell lines (Figure 4.12). 


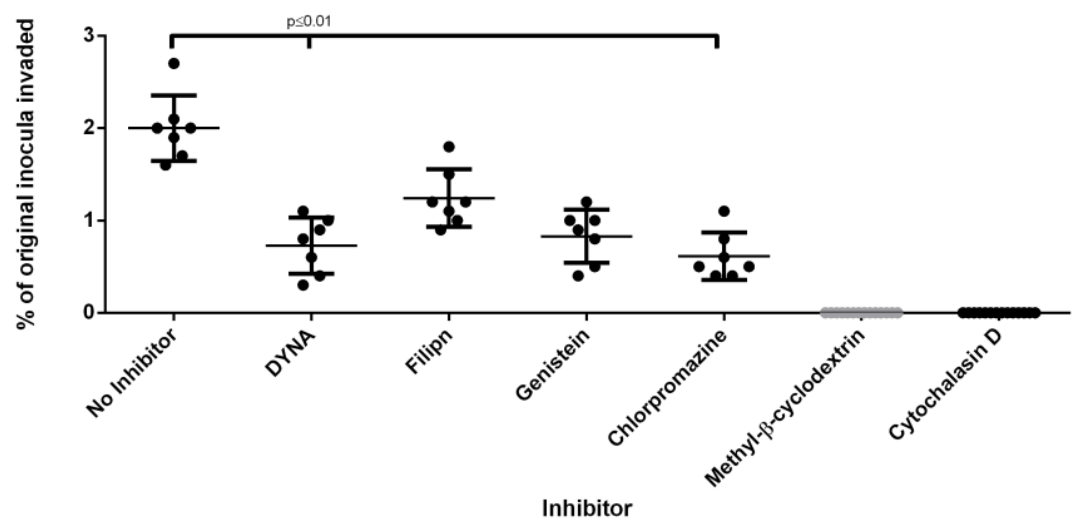

b

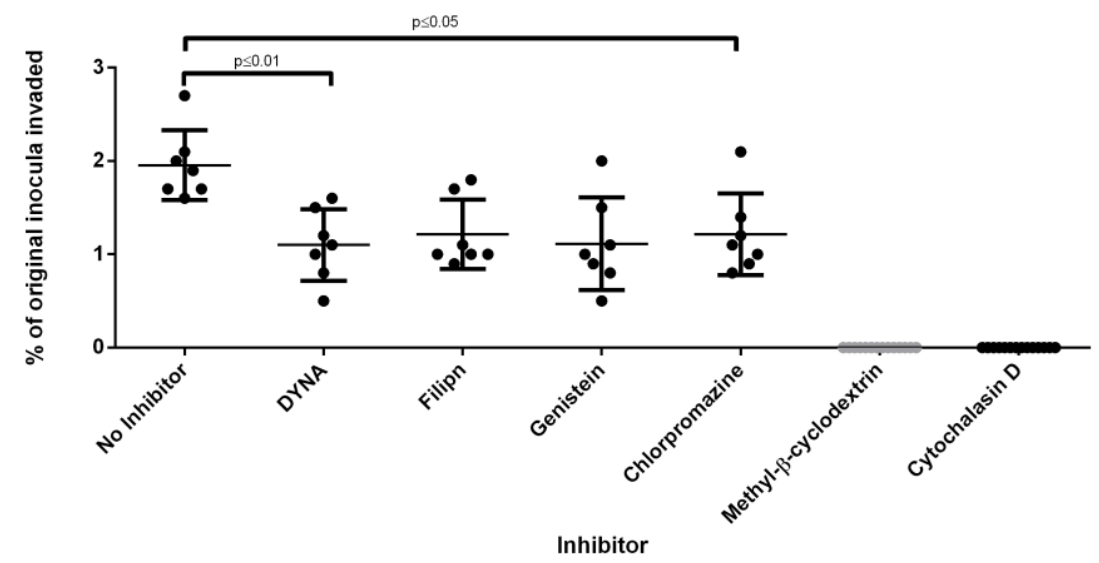

Figure 4.11 The effect of inhibition of cellular endocytosis on $C$. jejuni invasion into epithelial cells; (a) HT-29 and (b) 8E11 cells were pre-treated with endocytosis inhibitors prior to infection with $C$. jejuni for 6 hours and then $C$. jejuni invasion was assessed. Each dot represents three biological replicates in one strain. Results are also expressed as mean +/- SD of all strains measured. Differences were considered significant if $\mathrm{p} \leq 0.05$. 

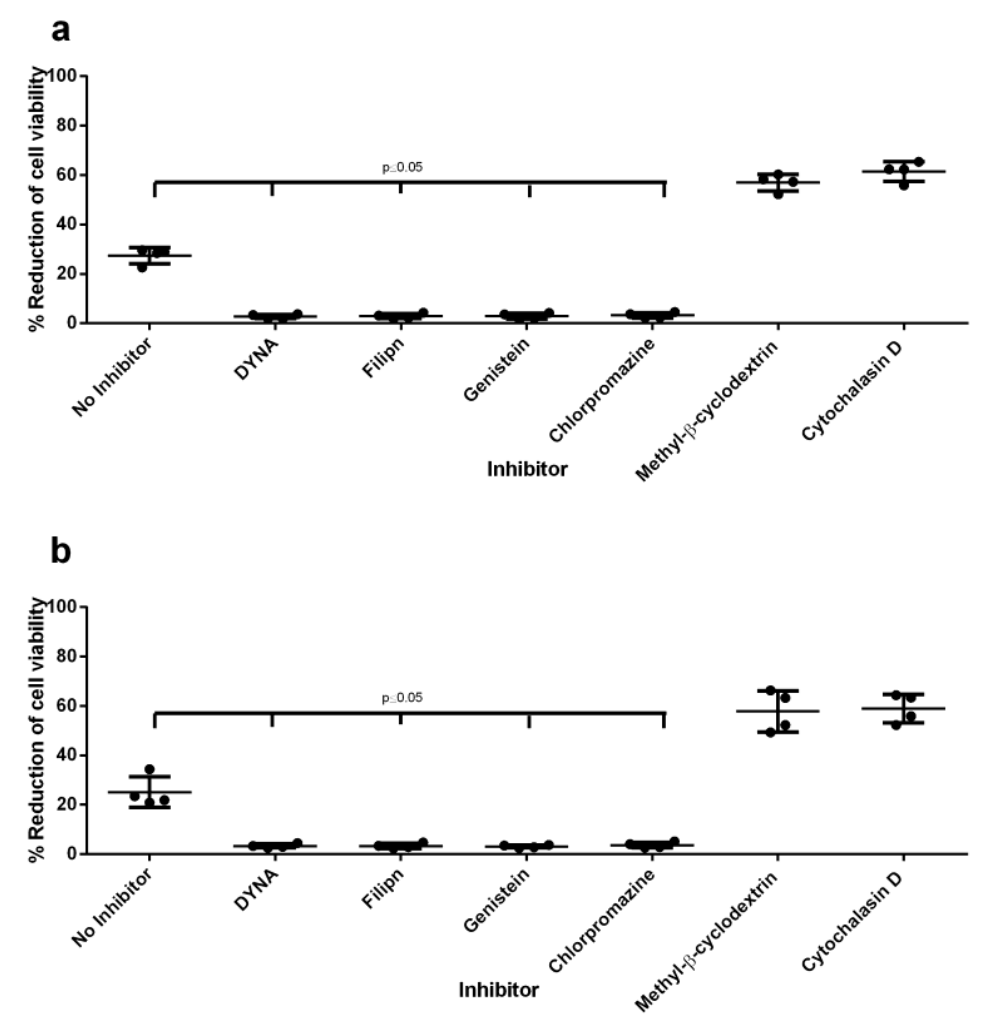

Figure 4.12 The effect of inhibition in the reduction of cell viability of epithelial cells after infection with $C$. jejuni; (a) HT-29 and (b) 8E11 cells were pre-treated with endocytosis inhibitors prior to infection with $C$. jejuni for 6 hours and then $C$. jejuni invasion was assessed. Each dot represents three biological replicates in one strain. Results are also expressed as mean $+/-$ SD of all strains measured. Differences were considered significant if $\mathrm{p} \leq 0.05$.

\subsubsection{C. jejuni-induced IL-8 and CXCLi1/2 expression is dynamin- and clathrin- dependent in human and chicken epithelial cells}

Cytokine expression was also determined following manipulation of endocytosis pathways. Consistent with invasion responses (Figure 4.11), IL-8 expression (Figure 4.13a) in human cells and CXCLi1 (Figure 4.13b) and CXCLi2 (Figure 4.13c) expression in chicken cells was dependent on dynamin and clathrin (Figure 4.13). In contrast to invasion responses, inhibition of caveolin pathways also significantly reduced IL-8, CXCLi1 and 2 expression. Cytokine expression could be detected in the absence of toxicity and with minimal invasion responses (Figures 4.11 and 4.13). These results confirm the importance of clathrin, caveolin and dynamin for $C$. jejuni-induced IL-8 production. 


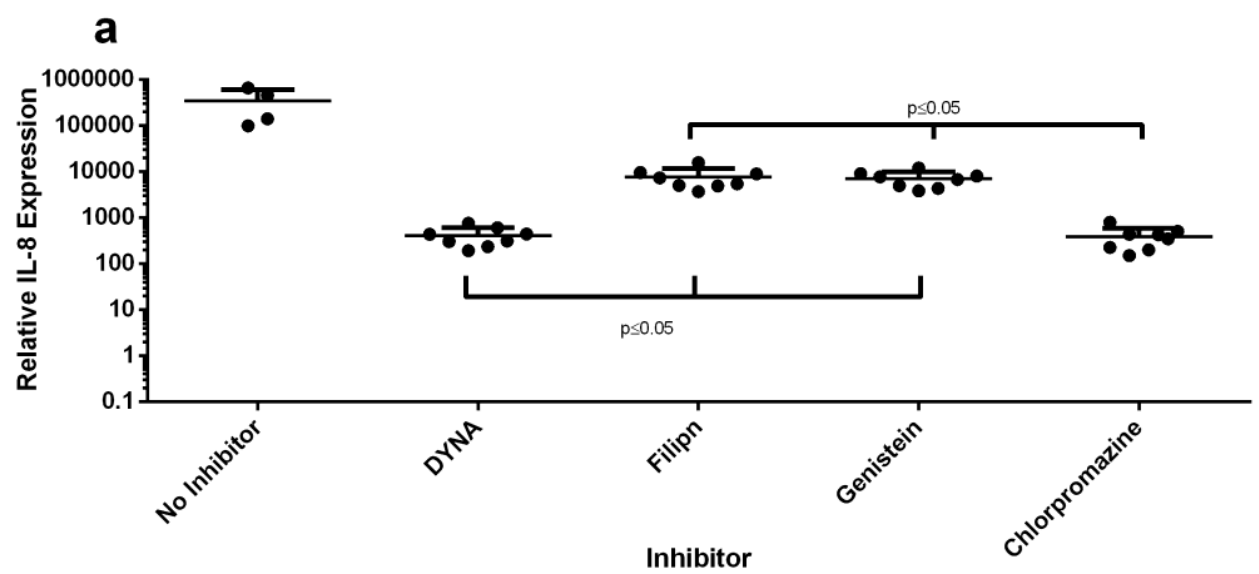

b

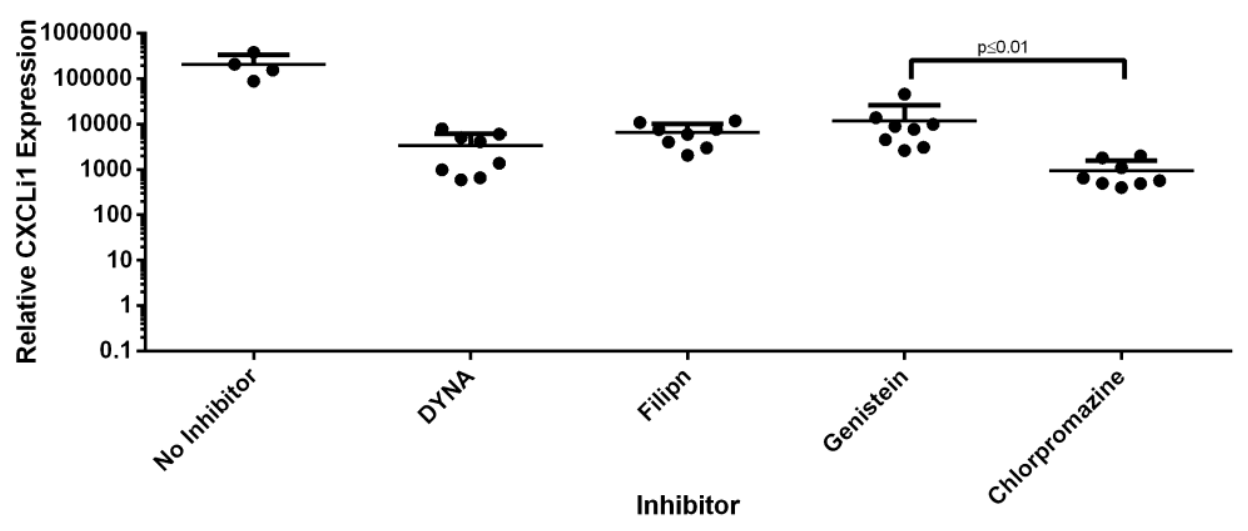

C

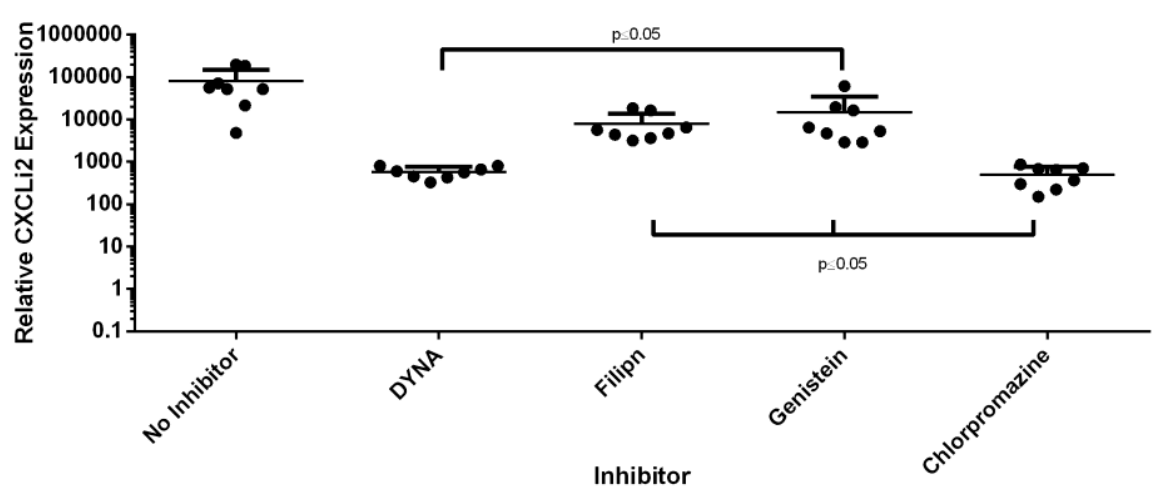

Figure 4.13 The effect of inhibition of endocytosis of cytokines cytokine expression in epithelial cell;. (a) HT-29 and (b, c) 8E11 cells were treated with endocytosis inhibitors prior to infection with $C$. jejuni for 24 hours. Then total RNA was isolated and specific mRNA was quantified by qPCR. Expression of (a) IL-8, (b) CXCLi1 and (c) CXCLi2 in epithelial cells. Each dot represents three biological replicates in one strain. Results are also expressed as mean $+/-$ SD of all strains measured. Differences were considered significant if $\mathrm{p} \leq 0.05$. 


\subsubsection{GWAS analysis of strains with high and low invasion and expression of immune markers}

Scoary analysis (as outlined in chapter 3) was used to investigate genes associated with in vitro responses in this chapter. Responses were categorised into 'high', 'intermediate' and 'low' and then GWAS was performed. A total of 7 genes were found to be associated with high expression of IL-8/CXCLi1/2 from human and chicken epithelial cells including motility linked flagellar hook protein, flgE. A further 5 genes were found to be associated with the average, intermediate expression and 8 with low expression (Table 4.14). Scoary identified 2 genes which were associated with high expression of IL-10 with a further 11 related to intermediate level of expression and 10 genes associated with low level expression (Table 4.15). The genes $h d d A$ and gmhA were associated with both the high and intermediate expression of IL-10, with porA, a major outer membrane protein also found associated.

Scoary was next used to investigate genes associated with the invasion response in both human and chicken epithelial cells (Table 4.16). A total of 4 genes were associated with the highest levels of invasion into epithelial cells including an anaerobic transporter. Another 4 genes were associated with intermediate levels of invasion and 9 associated with the lowest levels of invasion (Table 4.16). 
Table 4.24. Genes found to be related to expression of IL-8/CXCLi1/2.

\begin{tabular}{|c|c|c|c|c|}
\hline Gene & $\underline{\text { Locus Tag }}$ & $\underline{\text { Annotation }}$ & $\frac{\frac{\text { Trait }}{\text { Positive }}}{\text { Isolates }}$ & p Value \\
\hline \multicolumn{5}{|c|}{ High IL-8/CXCLi1/2 } \\
\hline$\underline{u p p}$ & Cj1286c & Uracil phosphoribosyltransferase & 13 & $\underline{0.026}$ \\
\hline$m n m A$ & Cj0053c & Bacteriohemerythrin & $\underline{7}$ & $\overline{0.029}$ \\
\hline$f \lg E$ & Cj1729c & Flagellar hook protein FlgE & $\underline{7}$ & $\underline{0.038}$ \\
\hline$\underline{s e l U}$ & Cj0500 & tRNA 2-selenouridine synthase & $\underline{\underline{2}}$ & $\overline{0.026}$ \\
\hline$m c p 4$ & Cj0262c & Methyl-accepting chemotaxis protein 4 & $\underline{1}$ & $\underline{0.034}$ \\
\hline$\underline{k d p B}$ & $\underline{\mathrm{Cj} 0677}$ & $\begin{array}{l}\text { Potassium-transporting ATPase ATP- } \\
\text { binding subunit }\end{array}$ & $\underline{0}$ & $\underline{0.034}$ \\
\hline$\underline{r f b C}$ & Cj1430c & dTDP-4-dehydrorhamnose 3,5-epimerase & $\underline{0}$ & $\underline{0.035}$ \\
\hline \multicolumn{5}{|c|}{ Intermediate IL-8/CXCLi1/2 } \\
\hline$\underline{s b p}$ & Cj0256 & $\underline{\text { Sulfate-binding protein }}$ & $\underline{15}$ & $\underline{0.010}$ \\
\hline yagU & Cj0014c & $\underline{\text { Inner membrane protein YagU }}$ & $\underline{8}$ & $\underline{0.038}$ \\
\hline$\underline{k d p B}$ & $\underline{\mathrm{Cj} 0677}$ & $\begin{array}{l}\text { Potassium-transporting ATPase ATP- } \\
\text { binding subunit }\end{array}$ & $\underline{4}$ & $\underline{0.023}$ \\
\hline$\underline{\operatorname{cstA}}$ & Cj0917c & Carbon starvation protein $\mathrm{A}$ & $\underline{3}$ & $\underline{0.030}$ \\
\hline$\underline{m n m A}$ & Cj0053c & tRNA-specific 2-thiouridylase MnmA & $\underline{0}$ & $\underline{0.034}$ \\
\hline \multicolumn{5}{|c|}{ Low IL-8/CXCLi1/2 } \\
\hline yedZ & $\underline{\mathrm{Cj} 0379 \mathrm{c}}$ & $\begin{array}{l}\text { Sulfoxide reductase heme-binding subunit } \\
\text { YedZ }\end{array}$ & $\underline{13}$ & $\underline{0.026}$ \\
\hline fliY & $\underline{\mathrm{Cj} 0059 \mathrm{c}}$ & L-cystine-binding protein FliY & $\underline{12}$ & $\underline{0.012}$ \\
\hline$\underline{\text { metF}}$ & $\underline{\mathrm{Cj} 1202}$ & 5,10-methylenetetrahydrofolate reductase & $\underline{12}$ & $\underline{0.030}$ \\
\hline$\underline{m d a B}$ & Cj1545c & Modulator of drug activity B & $\underline{12}$ & $\underline{0.030}$ \\
\hline$\underline{\operatorname{selB}}$ & $\underline{\mathrm{Cj} 1379}$ & $\underline{\text { Selenocysteine-specific elongation factor }}$ & $\underline{12}$ & $\underline{0.030}$ \\
\hline$\underline{f l g} S$ & $\underline{\mathrm{Cj} 0793}$ & $\begin{array}{l}\text { Signal transduction histidine-protein kinase } \\
\underline{\text { BaeS }}\end{array}$ & $\underline{11}$ & $\underline{0.002}$ \\
\hline folP & $\underline{\mathrm{Cj} 0585}$ & $\begin{array}{l}\text { Bifunctional dihydropteroate } \\
\text { synthase/dihydropteroate reductase }\end{array}$ & $\underline{11}$ & $\underline{0.006}$ \\
\hline cysA & $\underline{\mathrm{Cj} 0300 \mathrm{c}}$ & $\begin{array}{l}\text { Sulfate/thiosulfate import ATP-binding } \\
\text { protein CysA }\end{array}$ & $\underline{11}$ & $\underline{0.023}$ \\
\hline
\end{tabular}


Table 4.35. Genes found to be related to expression of IL-10.

\begin{tabular}{|c|c|c|c|c|}
\hline Gene & Locus Tag & Annotation & $\begin{array}{l}\text { Trait } \\
\text { Positive } \\
\text { Isolates }\end{array}$ & p Value \\
\hline \multicolumn{5}{|c|}{ High IL-10 } \\
\hline$g m h A$ & $\mathrm{Cj} 1149 \mathrm{c}$ & Phosphoheptose isomerase & 1 & 0.00433 \\
\hline$h d d A$ & $\mathrm{Cj} 1425 \mathrm{c}$ & $\begin{array}{l}\text { D-glycero-alpha-D-manno-heptose 7- } \\
\text { phosphate kinase }\end{array}$ & 1 & 0.00463 \\
\hline \multicolumn{5}{|c|}{ Intermediate IL-10 } \\
\hline$m n m A$ & $\mathrm{Cj} 0053 \mathrm{c}$ & Bacteriohemerythrin & 11 & 0.02991 \\
\hline$h d d A$ & $\mathrm{Cj} 1425 \mathrm{c}$ & $\begin{array}{l}\text { D-glycero-alpha-D-manno-heptose 7- } \\
\text { phosphate kinase }\end{array}$ & 10 & 0.00952 \\
\hline$g m h A$ & $\mathrm{Cj} 1149 \mathrm{c}$ & Phosphoheptose isomerase & 10 & 0.01094 \\
\hline$y m f D$ & Cj1241 & Bacillibactin exporter & 8 & 0.01706 \\
\hline porA & Cj1259 & Major outer membrane protein & 8 & 0.04972 \\
\hline$r f b C$ & $\mathrm{Cj} 1264 \mathrm{c}$ & dTDP-4-dehydrorhamnose 3,5-epimerase & 7 & 0.00175 \\
\hline hyaD & $\mathrm{Cj} 1264 \mathrm{c}$ & Hyaluronan synthase & 5 & 0.04761 \\
\hline$f c l$ & $\mathrm{Cj} 1428 \mathrm{c}$ & GDP-L-fucose synthase & 4 & 0.03544 \\
\hline$h d d C$ & $\mathrm{Cj} 1431 \mathrm{c}$ & $\begin{array}{l}\text { D-glycero-alpha-D-manno-heptose 1- } \\
\text { phosphate guanylyltransferase }\end{array}$ & 2 & 0.03758 \\
\hline $\arg O$ & $\mathrm{Cj} 1715$ & Arginine exporter protein $\mathrm{ArgO}$ & 0 & 0.03116 \\
\hline \multicolumn{5}{|c|}{ Low IL-10 } \\
\hline ribF & Cj0589 & Riboflavin transporter & 8 & 0.01099 \\
\hline alsT & $\mathrm{Cj} 0905 \mathrm{c}$ & Amino-acid carrier protein AlsT & 8 & 0.03099 \\
\hline $\operatorname{carB}$ & $\mathrm{Cj} 0279$ & Carbamoyl-phosphate synthase large chain & 7 & 0.00372 \\
\hline$m d a B$ & $\mathrm{Cj} 1545$ & Modulator of drug activity B & 7 & 0.00873 \\
\hline proB & $\mathrm{Cj} 0097$ & Glutamate 5-kinase & 7 & 0.02698 \\
\hline$l d h$ & Cj1167 & L-lactate dehydrogenase & 7 & 0.02698 \\
\hline pyrC & $\mathrm{Cj} 0259$ & Dihydroorotase & 6 & 0.01728 \\
\hline ubiE & $\mathrm{Cj} 0324$ & $\begin{array}{l}\text { Ubiquinone/menaquinone biosynthesis C- } \\
\text { methyltransferase UbiE }\end{array}$ & 4 & 0.00437 \\
\hline tupB & $\mathrm{Cj} 1540$ & $\begin{array}{l}\text { Tungstate uptake system permease protein } \\
\text { TupB }\end{array}$ & 4 & 0.02367 \\
\hline $\operatorname{cobB}$ & $\mathrm{Cj} 1050 \mathrm{c}$ & NAD-dependent protein deacylase & 2 & 0.03099 \\
\hline
\end{tabular}


Table 4.46. Genes found to be related to the level of invasion into both human and chicken epithelial cells.

\begin{tabular}{|c|c|c|c|c|}
\hline Gene & $\begin{array}{l}\text { Locus } \\
\text { Tag }\end{array}$ & Annotation & $\begin{array}{l}\text { Trait } \\
\text { Positive } \\
\text { Isolates }\end{array}$ & p Value \\
\hline \multicolumn{5}{|c|}{ High Invasion } \\
\hline dcuA & Cj0088 & $\begin{array}{l}\text { Anaerobic C4-dicarboxylate transporter } \\
\text { DcuA }\end{array}$ & 6 & 0.033 \\
\hline hyaD & $\mathrm{Cj} 1264 \mathrm{c}$ & Hyaluronan synthase & 5 & 0.030 \\
\hline$f \operatorname{flg} R$ & $\mathrm{Cj} 1024 \mathrm{c}$ & $\begin{array}{l}\text { Sigma } 54 \text { associated transcriptional } \\
\text { activator }\end{array}$ & 2 & 0.031 \\
\hline $\operatorname{ars} B$ & $\mathrm{Cj} 1187 \mathrm{c}$ & Arsenical pump membrane protein & 2 & 0.031 \\
\hline purH & $\mathrm{Cj} 0953 \mathrm{c}$ & $\begin{array}{l}\text { Bifunctional purine biosynthesis protein } \\
\text { PurH }\end{array}$ & 2 & 0.040 \\
\hline \multicolumn{5}{|c|}{ Intermediate Invasion } \\
\hline ribF & Cj0589 & Riboflavin transporter & 8 & 0.011 \\
\hline$c d t A$ & $\mathrm{Cj} 0079 \mathrm{c}$ & Cytolethal distending toxin subunit A & 8 & 0.031 \\
\hline$m c r B$ & Cj0139 & $\begin{array}{l}\text { 5-methylcytosine-specific restriction } \\
\text { enzyme B }\end{array}$ & 3 & 0.041 \\
\hline upp & $\mathrm{Cj} 1286 \mathrm{c}$ & Uracil phosphoribosyltransferase & 2 & 0.011 \\
\hline \multicolumn{5}{|c|}{ Low Invasion } \\
\hline hisS & $\mathrm{Cj} 0765 \mathrm{c}$ & Histidine--tRNA ligase & 9 & 0.044 \\
\hline $\operatorname{tgt}$ & $\mathrm{Cj} 1010$ & Queuine tRNA-ribosyltransferase & 8 & 0.011 \\
\hline hypA & Cj0627 & $\begin{array}{l}\text { Hydrogenase/urease nickel incorporation } \\
\text { protein HypA }\end{array}$ & 8 & 0.011 \\
\hline$c g b$ & $\mathrm{Cj} 1586$ & Bacterial hemoglobin & 8 & 0.031 \\
\hline$f d h C$ & $\mathrm{Cj} 1509 \mathrm{c}$ & $\begin{array}{l}\text { Formate dehydrogenase, cytochrome } \\
\text { b556(fdo) subunit }\end{array}$ & 8 & 0.031 \\
\hline$n r f A$ & $\mathrm{Cj} 1357 \mathrm{c}$ & Cytochrome c-552 & 8 & 0.031 \\
\hline murG & Cj1039 & $\begin{array}{l}\text { UDP-N-acetylglucosamine--N- } \\
\text { acetylmuramyl-(pentapeptide) } \\
\text { pyrophosphoryl-undecaprenol N- } \\
\text { acetylglucosamine transferase }\end{array}$ & 7 & 0.027 \\
\hline$c y d B$ & Cj0082 & $\begin{array}{l}\text { 2-oxoglutarate-dependent } \\
\text { ethylene/succinate-forming enzyme }\end{array}$ & 2 & 0.011 \\
\hline selU & $\mathrm{Cj} 0500$ & tRNA 2-selenouridine synthase & 2 & 0.031 \\
\hline
\end{tabular}




\subsection{Discussion}

The data in this chapter add to the growing body of evidence that $C$. jejuni is a pathogen in chickens (Byrne et al., 2007; Humphrey et al., 2014; Jennings et al., 2011; Neill et al., 1984; Ruiz-palacios et al., 1981; Sanyal et al., 1984; Williams et al., 2013). C. jejuni induce inflammatory and toxicity responses and can also invade human and chicken epithelial cell lines. While there was little difference between human and chicken cell responses, there was a wide range across all bacterial strains studied. The 100 strains of C. jejuni analysed were deliberately chosen from a variety of sources (including chicken, human, cattle and wild birds) and across sequence types to give a good representation of strains studied previously at the genomic level although we did not detect differences between these groups (Sheppard et al., 2011, 2013). Coincidentally, the reference strains M1 and NCTC11168 produced responses equivalent to the average for the whole study population. The question remains as to the source of the variation across the whole population. Individual virulence factor expression was not measured in this study but previous work on the transcriptome of a variety of Campylobacter strains showed that they are subject to complex regulation (Dugar et al., 2013).

Epithelial-derived IL-8 production is vital for early neutrophil infiltration into the gut in humans (Bennett Jr. et al., 2010) and chickens (Humphrey et al., 2014). This study confirms the importance of human IL-8 and identifies both CXCLi1 and CXCLi2 as important early chemokines induced in chicken epithelial cells following $C$. jejuni infection. This is consistent with studies in chicken LMH epithelial cells (Larson et al., 2008). Interestingly, CXCLi2 (like human IL-8) was significantly higher than CXCLi1. This is the first time that a study using a large collection of $C$. jejuni strains has found differences in these two chicken IL-8 homologues. Other studies found no differences (Kogut et al., 2005) but on a smaller scale (n=6 strains) and Larson et al., (2008) found the opposite effect. It is interesting to speculate that CXCLi2 (and not CXCLi1) may be the functional equivalent to IL-8 in humans. This is supported by structural data on amino acid similarity where CXCLi1 and CXCLi2 are 48 and 67\%, respectively, identical to human IL-8 (Gupta et al., 2008; Sick et al., 2000). Expression of IL-8 mRNA and IL-8 protein yielded different patterns. Expression of mRNA does not always correlate with protein expression with not all mRNA converted into protein with work by (Shebl et al., 2010). 
The three cellular responses defined by 'IL-8 production', 'cellular toxicity' and 'Campylobacter invasion' are inextricably linked, as demonstrated by the correlation curves (Figure 4.10). Clear links were demonstrated between increased cytokine output (IL-8, CXCLi1 and 2) and 'toxicity' suggesting similar mechanisms of induction. Indeed, Hickey and co-workers suggested two mechanisms of Campylobacter-induced IL-8 production involving; i) adherence and or invasion; and ii) $c d t$ expression (Hickey et al., 1999, 2000). While this known link between IL-8 and invasion was demonstrated in INT407 cells, we did not find a significant correlation between them in HT-29 cells. In contrast, strong positive correlations were made in chicken cells between both CXCLi1/2 and invasion, again supporting an important role for these cytokines in early Campylobacter responses in chickens.

Numerous human cell lines have been used to study Campylobacter pathogenesis, including T84 (Zheng et al., 2008), INT407 (Borrmann et al., 2007), HT-29 (Bahrami et al., 2011), and Caco-2 (Man et al., 2010) intestinal epithelial cells. There are very few data on chicken intestinal cell systems, due to the lack of commercially available cell lines until recently when one company (MicroMol) produced several clones. These cell lines are particularly useful for high throughput studies with many strains such as the use of them for this purpose in the current study. When considering the three cellular endpoints of IL-8 expression, cellular toxicity, and Campylobacter invasion none of these cells mimic equivalent in vivo responses exactly. Campylobacter invasion into Caco-2 cells show very good correlation to in vivo invasive potential in chickens (Hanel et al., 2004) and humans (Everest et al., 1992) but produce limited cytokine responses (MacCallum et al., 2006) whereas HT-29 and T84 produce robust cytokine responses but no good evidence of relevance to in vivo invasion responses (MacCallum et al., 2006). In the present study, cellular invasion in both HT-29 cells and the 8 E11 chicken cells at a level of $1-3 \%$ of the initial inoculum with sufficient robustness to differentiate strains and for consistency over three replicate experiments was detected. This is in keeping with levels of invasion of up to $4 \%$ in Caco- 2 cells shown previously (Hanel et al., 2004). This suggests great potential in the chicken cell line used in this study for investigating the diversity of $C$. jejuni responses in vitro.

This study confirmed the importance of canonical pro- (ERK) and anti-inflammatory (PI 3-Kinase -Akt) pathways for the C.jejuni-induced production of IL-8 and IL-10 in 
human epithelial cells (Li et al., 2011; Watson and Galan, 2005). Furthermore, ERKdependent Campylobacter invasion is also supported by previous studies ( $\mathrm{Hu}$ et al., 2006a; Jin et al., 2003; Samuelson et al., 2013; Samuelson and Konkel, 2013). The importance of these pathways in chicken cells is a novel result of this study and confirms that the underlying mechanisms are similar between human and chicken cells. In addition, the significantly higher IL-10 responses in the chicken cells suggest that the chicken gut may produce IL-10 as a method to tolerate large doses of Campylobacter and is supported by previous in vivo studies showing that some breeds of chicken produce more 'regulated' responses (Humphrey et al., 2014). While the chicken cells used here are derived from leghorn chickens it would be interesting to speculate on the breeds used previously (Humphrey et al., 2014) as there is evidence using Bayesian structural modelling of in vivo responses in chickens that IL-10 profiles are indeed different between breeds and this needs exploring further through cell line models derived from broiler chickens (Reid et al., 2016; Williams et al., 2013).

Campylobacter uptake by endocytic pathways confirmed the requirement of lipid rafts (Lin et al., 2011) in human cells and extended this role to chicken epithelial cells. This study also found that microfilaments were required for invasion of both HT-29 cells and the chicken ones. Interestingly, at least two mechanisms exist as INT407 (Konkel and Cieplak Jr., 1992) and Caco-2 (Russell and Blake, 1994) cells show microfilaments/microtubules-dependent and -independent mechanisms, respectively, suggesting that the role of cytoskeleton may be strongly cell dependent. Further work into mechanisms of uptake, in the present study, confirmed roles for dynamin and clathrin which has not been documented to date. Interestingly Cdt uptake into cells does involve clathrin coated pits (Thelestam and Frisan, 2004) and may be the mechanisms observed here. A consistent role for caveolins (using filipin and genistein) in the uptake process could not be confirmed during this study. Indeed, this is consistent with a recent publication which suggests that $C$. jejuni invasion is independent of caveolins (Konkel et al., 2013).

Throughout this chapter many of the unknown mechanisms highlighted in figures 1.4 and 4.1 have been identified (Figure 4.14). C. jejuni has been shown to effectively invade into host cells causing inflammatory markers to be expressed and disrupt the 
immune response in the host with $C$. jejuni shown to use similar signalling pathways to the human host (Figure 4.14).

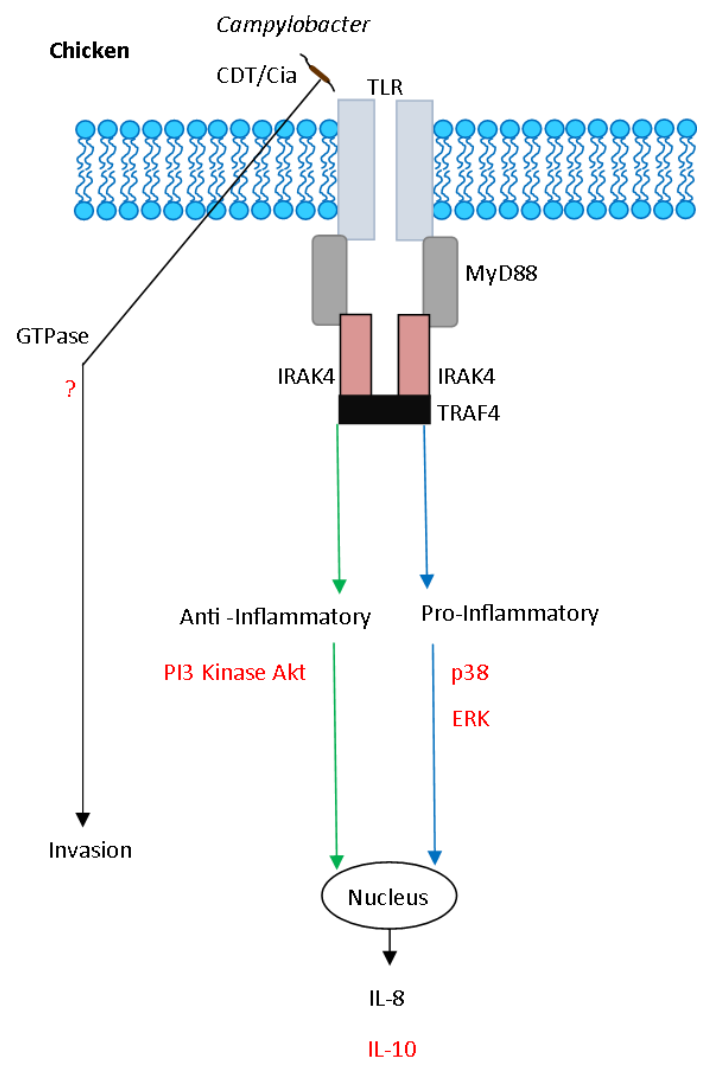

Figure 4.14 Identified aspects of the chicken system during this chapter

One interesting consequence of inhibiting endocytic pathways was the concurrent reduction in cell toxicity confirming that Campylobacter is responsible for the toxicity and the endocytic inhibitors have negligible effects on cell viability, however; inhibitors such as Methyl- $\beta$-cyclodextrin and cytochalasin D were shown to be very toxic to both cell lines and so lower doses in future experiments would be needed to confirm that the differences observed are not due to the viability of the cells being reduced. Another consequence of inhibiting endocytosis was the 'extra' effect of inhibiting cytokine production. Previously, de Zoeta and co-workers (De Zoete et al., 2010) established that live Campylobacter are very weak stimulators of both human and chicken TLR-2, -4 and -5. In striking contrast, lysed Campylobacter induce strong NFkappaB activation through human TLR1/2/6 and TLR4 and chicken TLR2t2/16 and TLR4 but not via TLR5 of either species (De Zoete et al., 2010). The results of the current study, support the concept that 'invasion' or 'internalisation' of some kind is 
necessary for cytokine production. Indeed, Hickey et al also suggest an 'invasion' dependent pathway for IL-8 induction (Hickey et al., 2000). Preliminary TLR expression data displayed the presence of many TLRs on both the human and chicken cell lines, however further work is needed to expand the TLR profiles of the novel 8E11 chicken cells (Figure 4.15).
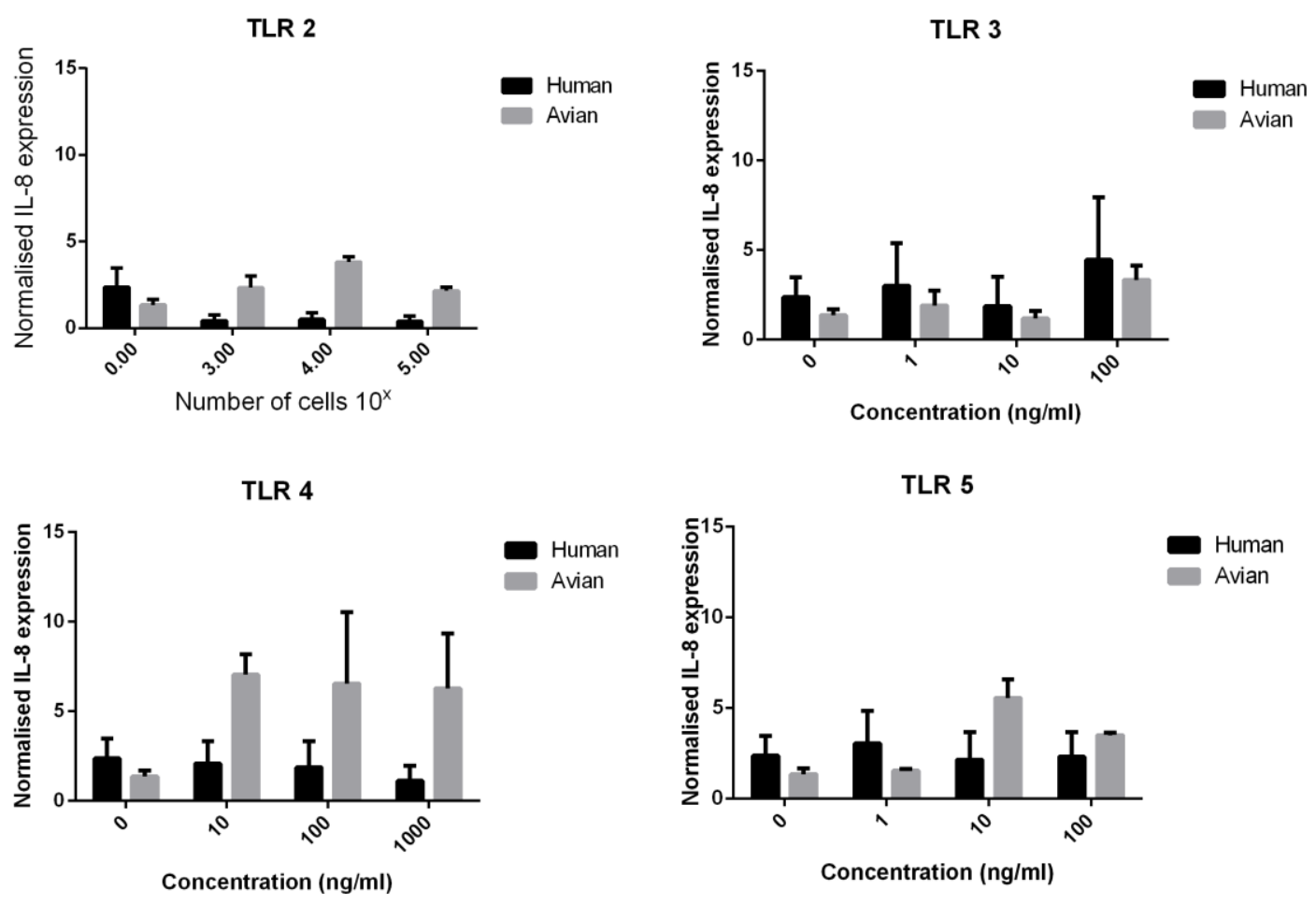

Figure 4.15 Preliminary experiment where HT-29 and 8E11 cells were cultured with ligands to show expression of TLRs in both systems.

These results are novel because of the number and diversity of relevant $C$. jejuni strains used with an chicken cell line to determine pathogenic mechanisms. However, there are certain limitations that provide an opportunity to improve the work in the future. Firstly, chicken 8E11 cells are derived from s intestines of embryonic white leghorn chickens and are positive for enterocyte markers, villin, E-cadherin and cytokeratin. Sequencing of the 8E11 cells found a small amount of quail DNA within the cells, thus expression of the chicken specific MHCs was used as a marker due in part to work by Shiina et al (2004) who found that quail and chickens have different MHCs led us to confidently call the MM-ChiC clone 8E11 cells chicken intestinal cells (Shiina et al., 2004). Previous studies have confirmed the importance of broiler breed to the final 
inflammatory response and suggest that cells from commercial fast and slower growing breeds may be more relevant to study chicken gut responses (Kaiser et al., 2016). Indeed, recent technologies point to the precision modelling of chicken intestinal slices (Punyadarsaniya et al., 2015). Secondly, we focused our attention on using C. jejuni strains that define the species, from a variety of sources and sequence types, as published previously (Sheppard et al., 2013). Recent Campylobacter outbreaks have highlighted the importance of invasion from the gut (e.g. to the liver) suggesting the importance of focussing on groups of invasive and non-invasive Campylobacter in future studies. Under-cooked chicken muscle is also a highly important vehicle for human infection internationally.

In this chapter a new commercially avaiable chicken cell line was used to determine the inflammatory responses in chickens while also investigating the signalling pathways $C$. jejuni uses during infection into host cells. In the next chapter, methods from chapters 3 and 4 will be used to analyse Campylobacter strains from naturally and experimentally infected chickens to investigate genomic and phenotypic differences in strains from different sections of the chicken gut and also investigate potential biomarkers in chickens.

GWAS analysis to find association between $C$. jejuni genes and expression of immune markers or invasion responses in human and chicken epithelial cells identified many potential genomic markers including $f l g E$, a motility linked gene, which was associated with high levels of IL-8/CXCLi1/2 expression which was also found to be related to strains from human sources in chapter 3. The gene upp, was found to be associated with high level of of IL-8/CXCLi1/2 expression and also intermediate level of invasion into epithelial cells which could help to support the link between IL-8 production and the invasiveness of Campylobacter (Hickey et al., 1999; Zheng et al., 2008). The genes $g m h A$ and $h d d A$ were found to be related to high levels of IL-10 expression, these genes are required for the synthesis of heptose (Guerry et al., 2012). Wong et al (2015) suggested that a modified heptose may lead to bacterial resistance against gastrointestinal host defenses and can support the persistence of Campylobacter and thus the genes gmhA and $h d d A$ being associated to IL-10 may be due to the modified heptose triggering IL-10 expression to form an anti-inflammatory response to counteract gut inflammation (Wong et al., 2015). 


\subsection{Conclusion}

In conclusion, these novel data suggest that chicken systems are likely to use similar host defence pathways to humans in response to Campylobacter spp. Strong correlation was found between IL-8 with invasion and toxicity in the human cells. However, the diversity and range of responses suggests that 'a one strain fits all approach' to in vivo experimental infection would not give meaningful data for the study of Campylobacter pathogenesis. The novel chicken cell line, 8E11, was demonstrated to be a good candidate for a model to investigate the chicken system with the host responses measured suggesting that chicken and human epithelial cells share common mechanisms to combat $C$. jejuni with exceptional phenotypic diversity. GWAS analysis was able to find genes assocated with expression of IL-8, IL-10 and level of invasion. 


\section{Chapter 5 - Host responses to Campylobacter strains from naturally and experimentally infected chickens}

\subsection{Introduction}

\subsubsection{Campylobacter and chicken production}

Poultry, in commercial terms, includes chicken, turkey, duck and laying hens. Chicken is the predominant species used in the meat production industry comprising $70-80 \%$ of the animals used (Skarp et al., 2016). Chicken is thus a very important source of relatively cheap but nutritionally high quality protein worldwide; with production now being industrialised in many developing countries, particularly in SE Asia. In the UK alone, approximately one billion chickens are reared for meat consumption annually, with around $75 \%$ of them being positive for Campylobacter at point of sale (PHE, 2016). Estimates suggest that chickens account, either directly and indirectly, for up to $80 \%$ of human Campylobacter infections (Skarp et al., 2016).

Poultry production has a pyramid structure. There are a few breeding companies at the top and they supply producers with birds for breeding flocks. These provide chicks for primary production at rearing farms. When the animals reach slaughter weight, usually $2.2 \mathrm{Kg}$, they are transported to the abattoir. The chickens are either left as carcasses or processed into products, which are sold at retail and/or enter the catering industry. These stages play a part in the transmission of Campylobacter from farm to fork. The prevalence of Campylobacter-positive broiler flocks in the EU varies between countries and regions. It is low in Scandinavia and ranges from $0.6-13.1 \%$ in Norway, Sweden and Finland,. In other countries such as France and the UK up to 74-80\% of flocks are positive (EFSA, 2010; Skarp et al., 2016). Flocks in commercial production usually consist of approximately 10,000 to 50,000 birds per house; with several houses often present on a farm potentially facilitating the spread of Campylobacter from house to house for rapid spread through the farm. Flocks can be infected by one or multiple strains simultaneously. At the retail end of the processing chain there are various products on sale from whole carcasses to different cuts and portions, as well as fresh and frozen products which pose different infection risks. Campylobacter can be found on skin (Hansson et al., 2015; Marotta et al., 2015), in muscle (Hansson et al., 2015; Scherer et al., 2006) and in liver tissues (Barot et al., 1983; Humphrey et al., 2015; Lahti et al., 2017; Whyte et al., 2006). 


\subsubsection{Campylobacter in chickens}

Past dogma suggested that Campylobacter behaved as a commensal in chicken hosts (Brisbin et al., 2008; Connell et al., 2012). However, work going back to the 1980s and more recent studies (Humphrey et al., 2014; John et al., 2017; Ruiz-palacios et al., 1981; Sanyal et al., 1984; Williams et al., 2014), as well as work conducted in vitro in Chapter 4 of this thesis, have demonstrated that Campylobacter can behave as a pathogen in chickens. However, the virulence mechanisms responsible for such behavioiurs of Campylobacter strains are not yet understood completely (Byrne et al., 2007; Humphrey et al., 2014; Jennings et al., 2011; Williams et al., 2013).

\subsubsection{Campylobacter extra-intestinal spread}

In recent years, epidemiological evidence has suggested strongly that the greater human health threat is the contamination/infection of liver and muscle tissues (Lahti et al., 2017; Rosner et al., 2017) compared to Campylobacter on chicken carcass surfaces. In the UK, an industry-wide campaign to reduce the percentage of carcasses entering the food chain, which has been very successful, has had no significant impact on the number of human cases (PHE and FSA data 2018). Campylobacter have been shown to be highly heat resistant when attached to chicken muscle tissues, contributing to an infection risk in undercooked chicken muscle, and infected chicken liver is also a major source of infections in humans (De Jong et al., 2012; Lahti et al., 2017; Rosenquist et al., 2009; Rosner et al., 2017; Whyte et al., 2006). Despite isolation from edible tissues the mechanism by which Campylobacter can transmigrate from the chicken gut to liver and muscle tissues is not well defined (Berntson et al., 1992; Hansson et al., 2015; Humphrey et al., 1993; Luber and Bartelt, 2007; Scherer et al., 2006).

\subsubsection{Caecal Tonsils}

The caecal tonsils are an important component of the chicken GALT, which plays a significant role in inducing and regulating immune responses locally and systemically (Oláh et al., 2013). Caecal tonsils are a cluster of aggregated lymphoid tissue, which is located within the medial wall of caeca at its transition to the rectum (Casteleyn et al., 2010; Gómez Del Moral et al., 1998). Caecal tonsils are composed of several subunits made up of secondary lymphoid follicles with interspersed $\mathrm{T}$ cell regions which are located around a central fossula (Kitagawa et al., 1998). The fossula itself branches into several crypts, which connect directly to the caecal lumen, with $\mathrm{M}$ cells scattered between columnar epithelial cells that are present on a lympho-epithelium which lines 
the crypts (Casteleyn et al., 2010; Kitagawa et al., 1998). The subunits of the tonsils are separated from each other by septa of connective tissue (Kitagawa et al., 1998). Caecal tonsils appear late in the embryonic stage of development. However, only after hatching does their first major development occur. The first day after the chick has hatched; both B and T lymphocytes are formed. After 6 weeks post-hatching the number of B cells far exceeds those of T lymphocytes (Gómez Del Moral et al., 1998). When the chicken is fully matured, the caecal tonsils are large and so represent a major component of its GALT. As a result of their large size caecal tonsils can be extracted easily and used to investigate the local immune responses in the chicken gut (Haghighi et al., 2008; Janardhana et al., 2009; Yurong et al., 2005).

The exact function of the chicken caecal tonsil is not certain. However, some authors suggest that they may neutralise antigens that enter the caeca due to reflux of urates (Kitagawa et al., 1998). Other investigators suggest that the caecal tonsils and other chicken GALTs might play a role in the differentiation of stem cells into B lymphocytes (Befus et al., 1980).

\subsubsection{Immune biomarkers in chickens}

Important inflammatory markers in the chicken immune response include ILs-8, -10, $1 \beta$ and TNF $\alpha$. IL- 8 is an important mediator in the innate immune response, which attracts neutrophils and other granulocytes to a site of infection and can induce phagocytosis (Yoshimura, 2015). In the chicken system, there are two orthologues for CXCLi1 and CXCLi2 (Larson et al., 2008). Studies have shown that C. jejuni can stimulate the production of both CXCLi1 and CXCLi2 (Larson et al., 2008; Tahaabdelaziz et al., 2016). Another pro-inflammatory cytokine, tumour necrosis factor alpha (TNF- $\alpha)$, is also involved in the innate immune response and is produced primarily by macrophages but also by many other cell types including neutrophils, CD4+ lymphocytes, mast cells and natural killer (NK) cells. The primary role of TNF- $\alpha$ is regulation of immune cells (Olmos and Lladó, 2014) with Campylobacter being able to induce activation of TNF- $\alpha$, which may subsequently activate NF-k $\beta$ (Namin et al., 2015; Olmos and Lladó, 2014; Rohde et al., 2018).

Aquired immunity in chickens offers protection against the development of bloody diarrhoea in response to infection with $C$. jejuni. T lymphocytes such as the $\mathrm{CD} 4^{+}$act as $\mathrm{T}$ helper $\left(\mathrm{T}_{\mathrm{H}}\right)$ cells which recognise antigens associated with MHC II, which can be 
further divided into $T_{h} 1, T_{h} 2, T_{h} 17$ and $T_{h} 22$ responses. $T_{h} 1$ cells develop in response to the innate immunity and activate macrophages to produce $\operatorname{IgG}$ antibodies to promote phagocytosis and also produce TNF to promote leukocyte recruitment and inflammation. $\mathrm{T}_{\mathrm{h}} 2$ cells provide helper functions for $\mathrm{B}$ cells, stimulating production of antibodies and inhibit macrophage activity thus are considered anti-inflammatory and regulatory to control $T_{h} 1$ pro-inflammatory responses. $T_{h} 17$ cells contribute to propagation of an inflammatory response and provide mucosal immunity. $\mathrm{T}_{\mathrm{h}} 22$ cells are linked to pro-inflammatory responses in the gut and wound healing (Al-Banna et al., 2018).

An important cytokine, which has a function in stimulating the immune response is IL-6 produced in response to infection as well as tissue injuries and contributes to host defence through stimulation of acute phase responses and immune reactions. IL-6 is secreted by macrophages and $\mathrm{T}$ cells, with $C$. jejuni recognition activating a MyD88independent secretion via TLR-2 in Caco-2 cells and is expressed by chickens (Friis et al., 2009; Pielsticker et al., 2012; Tanaka et al., 2014).

Another important mediator of the inflammatory response is IL-1 $\beta$; produced by macrophages as a proprotein. It is involved in various cellular activities such as cell proliferation, differentiation and apoptosis (Palomo et al., 2015). C. jejuni increases IL$1 \beta$ gene transcription in chickens, with elevated levels of IL-1 $\beta,-6$ and -8 transcription in chicken cells infected with $C$. jejuni having the ability to activate innate immunity signalling pathways in chicken ileum explants (Fonseca et al., 2016; Li et al., 2008; Smith et al., 2005).

IL-10 plays an important role in the anti-inflammatory response in chickens by down regulating expression of T-helper cell cytokines and co-stimulatory molecules on macrophages (Couper et al., 2008; Walter, 2014). IL-10 expression in chickens increases immediately following infection with C. jejuni (Barjesteh et al., 2013). Interferon gamma (IFN $\gamma$ ) has an important role in both innate and adaptive immunity against some bacterial infections and is an activator of macrophages and induces class II major histocompatibility complex (MHC) molecule expression, IFN $\gamma$ is produced primarily by NK cells and natural killer T cells (Schoenborn and Wilson, 2007). The importance of IFN $\gamma$ comes from its immunostimulatory and immunomodulatory effects 
(Schoenborn and Wilson, 2007). IL-1 $\beta,-6,-10$ and IFN $\gamma$ play a role in the Th17 pathway which has been observed in birds infected with $C$. jejuni (Reid et al., 2016).

\subsubsection{Aim}

This chapter focuses on Campylobacter strains from naturally and experimentally infected chickens. Methods and results used in both Chapters 3 and 4 were used to investigate and discover any new potential biomarkers of infection using a collection of Campyloabacter strains from the chicken environment. This chapter will also:

- Characterise the genomes of bird-matched Campylobacter from different organs in naturally infected chickens

- Identify genes responsible for colonisation/infection of ileum, caeca and liver in naturally infected chickens

- Measure the immune response in caecal tonsils from experimentally infected chickens as a potential biomarker for Campylobacter infection

- Investigate the in vitro cytokine and invasion responses of Campylobacter strains used to experimentally infect chickens 


\subsection{Methods}

\subsubsection{Campylobacter strains from naturally infected chickens}

A collection of 96 Campylobacter strains, from a BBSRC project entitled 'Broiler gut health and $C$. jejuni infection: impacts of harvest management' (BB/M009610/1) were isolated from different anatomical locations of naturally infected chickens; the caeca, ileum and liver. A total of 42 of these 'bird-matched' Campylobacter strains were used during this study; 14 each from caeca, ileum and liver were used to infect human (Caco2) and chicken (8E11) epithelial cell lines. A full list of bacterial strains is in table 2.2 strains 101-142.

\subsubsection{Pan-Genome creation}

The pan-genome was created using Prokka (Seemann, 2014) and Roary (Page et al., 2015) as described in section 2.3. C. jejuni and C. coli strains were analysed in Prokka against species specific gene sets. Strains comprising a mix of $C$. jejuni and $C$. coli were ran against all Campylobacter genes. The resulting pan-genome was used to produce maximum likelihood phylogenetic trees using iTol and phandango (Hadfield et al., 2018; Letunic and Bork, 2016).

\subsubsection{GWAS using Scoary}

Scoary microbial pan-GWAS (Brynildsrud et al., 2016) was performed as described in section 2.3.3. Scoary was used to identify genes that were related to the different sources of the C. jejuni isolates (caeca, ileum, liver).

\subsubsection{Culture of Human and Chicken Intestinal epithelial cells}

Human and chicken cells were maintained and cultured as described in Chapter 2.2.3

\subsubsection{Infection studies}

The infection studies were performed as described in section 2.2.4

\subsubsection{Invasion Assay}

An invasion assay was performed on the 'bird-matched' strains listed in section 5.2.1, as described in section 2.2.13. During the invasion assay all six Campylobacter strains were also combined and added to the cell lines at a total of $1 \times 10^{7} \mathrm{cfu}$ to mimic the strain cocktail given in vivo. 


\subsubsection{RNA Isolation}

Total RNA was isolated from Caco-2 human or 8E11 chicken intestinal epithelial as described in section 2.2.6.

\subsubsection{Quantitative PCR of RNA transcripts}

Quantitative PCR was performed using the probe based method as described in section 2.2.7.

\subsubsection{PCR Conditions}

The AriaMX was run using conditions described in section 2.2.9.

\subsubsection{Chicken Study}

An in vivo chicken infection study was carried out as described in Chapter 2. Birds were infected with a cocktail of six Campylobacter (CJ13126, CJ12662, L4, I4, C8 and C15) strains using a litter seeding method (Sandilands et al., 2018) at a concentration of $1 \times 10^{5}$ c.f.u Campylobacter. The birds were infected by members of SRUC and Drs Williams and Wilkinson of Swansea Universty.

\subsubsection{Experimental infection}

For chicken infection, four strains; one isolated from the liver (L4), one from the Ileum (I4) and two from the caecum of infected chickens (C8 and C15) along with two known C. jejuni isolates (CJ13126 and CJ12662) were used to infect chickens during an in vivo chicken study described in section 2.5.1.

\subsubsection{RNA extraction from Caecal Tonsils}

Thirty $\mu \mathrm{g}$ of caecal tonsil was placed in $600 \mu \mathrm{l}$ of RNA lysis buffer with one $5 \mathrm{~mm}$ stainless steel bead (Qiagen, Crawley UK) and the tonsil disrupted using the FastPrep FP120 (Thermo scientific, Loughborough, UK) at 4 metres/sec for 1 minute. RNA was extracted subsequently using the QIAGEN RNeasy mini kit according to manufacturer's instructions. RNA was eluted in $50 \mu 1$ nuclease free water and stored at $20^{\circ} \mathrm{C}$ until needed and quantified using a NanoDrop (Thermo scientific, Loughborough, UK). 


\subsubsection{Statistics}

Normality was assessed using D'agostino-Pearson test. The two-way ANOVA with multiple comparisons and non-parametric Krustal-Wallis test, for multiple comparisons with post hoc Dunns test were used. Significance differences were accepted if $\mathrm{p} \leq 0.05$. Graphpad Prism 6.0 (San Diego, USA) was used to analyse and assess differences between treatment groups. 


\subsection{Results}

\subsubsection{Campylobacter strains from naturally infected chickens}

Forty two Campylobacter strains were isolated from the caeca, ileum and liver of commercial chickens sampled during the BBSRC project entitled 'Broiler gut health and C. jejuni infection: impacts of harvest management' (BB/M009610/1). The strains used in the studies described below came from 14 birds where each had Campylobacter in the caeca, ileum and liver; with common numbered birds taken from the same bird (Table 2.2; Strains 101-114).

\subsubsection{Genomic analysis of isolates from naturally infected chickens}

Roary was used to construct a pan-genome of the 'bird-matched' Campylobacter strains from naturally infected chickens (Figure 5.1 and 5.2). The phylogenetic tree created from the pan-genome displayed no clear pattern between isolates from the same bird and G11 and L11 and G7 and L7 were only isolates from the same bird to cluster together (Figure 5.1). Both reference isolates, M1 and 11168, clustered together (Figure 5.1). The resulting pan-genome contained 10446 genes, made up of 73 core ones $(99 \%$ $<=$ gene presence $<=100 \%)$, with an additional 156 in the soft core $(95 \%<=$ gene presence $<99 \%$ ) totalling 226 genes present in the core genome and 10217 in the accessory genome. In total this comprised 3093 shell genes $(15 \%<=$ gene presence < $95 \%)$ and 7124 cloud ones $(0 \%<=$ gene presence $<15 \%)$ (Figure 5.2). 


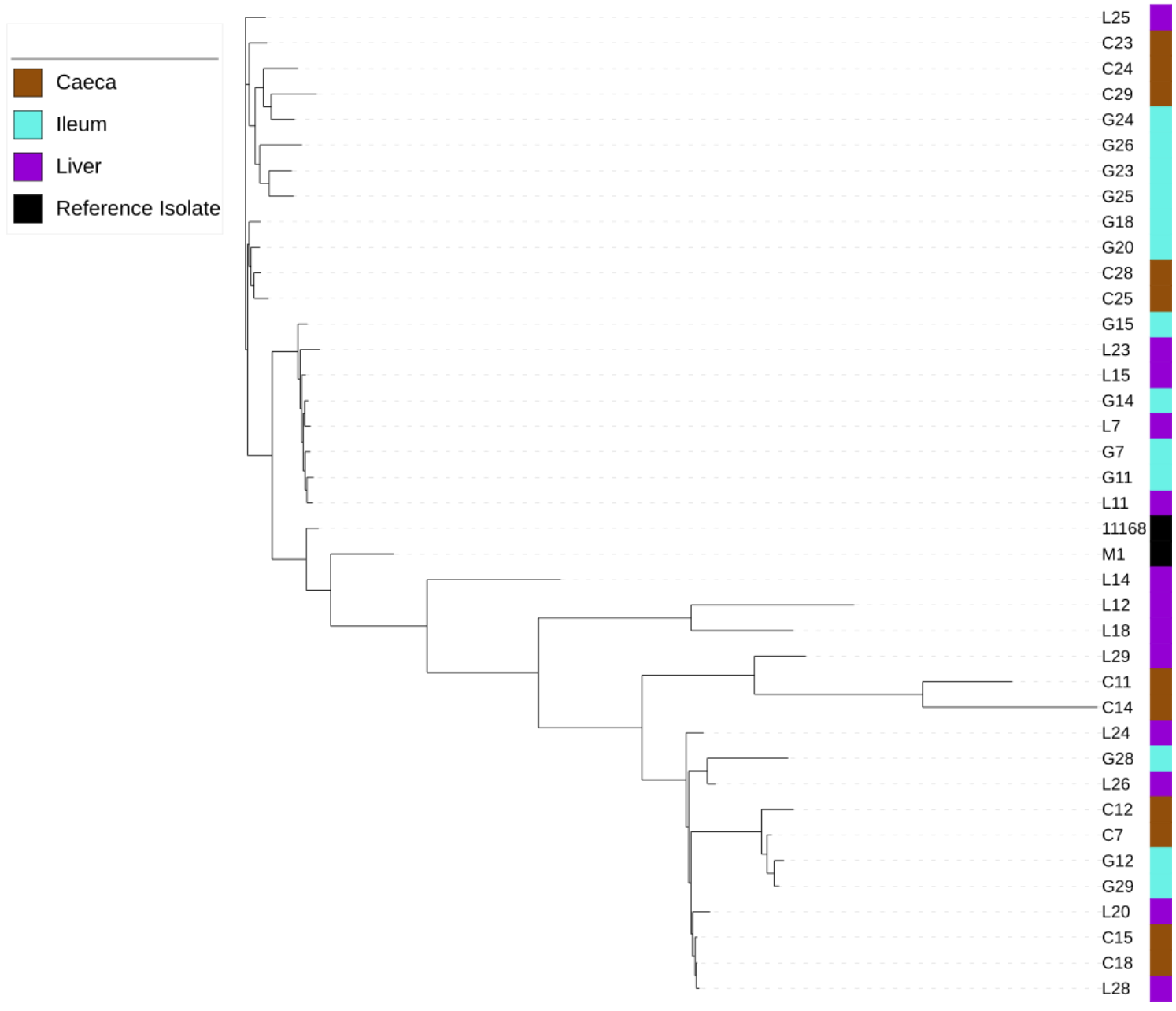

Figure 5.1. Maximum likelihood tree of the pan genome of Campylobacter isolates from the ceaca $(\mathrm{C})$, ileum $(\mathrm{G})$ and liver $(\mathrm{L})$ from naturally infected chickens with 11168 and $\mathrm{M} 1$ included as reference strains. 


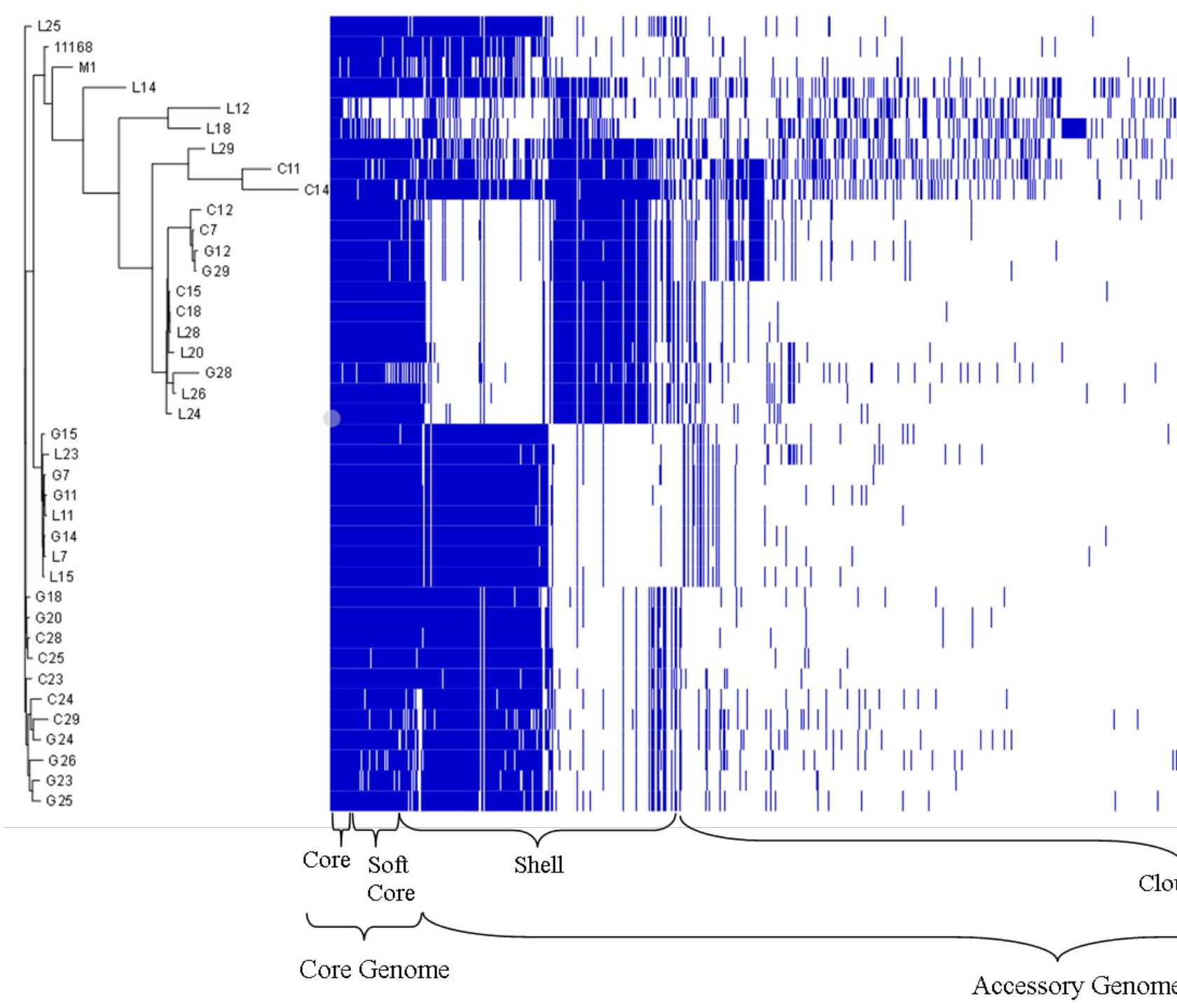

Figure 5.2. Representation of the pan-genome displaying the core and accessory genome with rel made up of the core genes (present in 99-100\% of isolates) and soft core ones (present in 95-99\% comprising shell genes (present in $15-95 \%$ of isolates) and the cloud genes (present in $0-15 \%$ of $i$ 


\subsubsection{Core Vs Accessory genome}

Analysis of the 42 isolates using the pan genome pipeline generated in Chapter 3 showed that the core genome totalled 226 genes. Of these, 38 were hypothetical proteins and so were removed from further analysis. The accessory genome contained 10217 genes from the shell and cloud, of which 4158 were hypothetical proteins and so were removed from further analysis (Figure 5.2).

Analysis of the known virulence genes from Chapter 3 (Table 3.1) showed the presence of two adhesion linked genes; pebAl and pldA, as the only known virulence linked genes in the core genome. All other genes were in the accessory genome (Table 5.1.) All known antimicrobial resistance linked genes were found in the accessory genome including; cmeB, aadE, tet $(O)$, bla $_{O X A-61}$ and aph-3-I.

Table 5.1. Presence of known virulence linked genes in the core and accessory genome

\begin{tabular}{|l|l|l|l|}
\hline Gene & Gene Name & Core & Accessory \\
\hline flaA & Flagellin A & & $\mathrm{x}$ \\
\hline flaB & Flagellin B & $\mathrm{x}$ \\
\hline flaC & Flagellin C & $\mathrm{x}$ \\
\hline flgS & Signal transduction histidine kinase & $\mathrm{x}$ \\
\hline flgR & Sigma-54 associated transcriptional activator & & $\mathrm{x}$ \\
\hline cadF & Outer membrane fibronectin-binding protein & & $\mathrm{x}$ \\
\hline capA & Auto-transporter protein & & $\mathrm{x}$ \\
\hline pebAl & Bi-functional adhesion/ABC transporter & $\mathrm{x}$ & \\
\hline aspartate/ glutamate-binding protein & & $\mathrm{x}$ \\
\hline peb4 3 & Major antigenic peptide PEB3 & Peptidyl-prolyl cis-trans isomerase & $\mathrm{x}$ \\
\hline pldA & phospholipase A & $\mathrm{x}$ & \\
\hline$j l p A$ & Surface exposed lipoprotein & & $\mathrm{x}$ \\
\hline ciaB & Campylobacter invasion antigen B & & $\mathrm{x}$ \\
\hline ciaC & Campylobacter invasion antigen C & $\mathrm{x}$ \\
\hline ciaI & Campylobacter invasion antigen I & $\mathrm{x}$ \\
\hline htrA & Serine protease & & $\mathrm{x}$ \\
\hline$i a m A$ & ABC transporter ATP-binding protein & $\mathrm{x}$ \\
\hline$f s p A$ & flagellum-secreted non flagellar protein & $\mathrm{x}$ \\
\hline cdtA & Cytolethal distending toxin A & $\mathrm{x}$ \\
\hline cdtB & Cytolethal distending toxin B & $\mathrm{x}$ \\
\hline cdtC & Cytolethal distending toxin C & \\
\hline
\end{tabular}




\subsubsection{GWAS of isolates from caeca, ileum and liver}

We applied the GWAS methods successfully performed in Chapter 3 (100 strain collection) and in Chapter 4 (in vitro pathogenesis in section 4.3.12) to investigate the association of genomic markers with 'organ / body compartment' in naturally infected chickens.

Scoary was used to investigate association between isolates from the caeca, ileum and liver. Scoary linked 60 genes to isolates from the caeca, excluding 137 hypothetical proteins. Among the most trait-positive isolates were three tRNA ligases (leuS, argS and valS), an outer membrane protein (ompA) and a macrolide export protein (macB) (Table 5.1). All 60 genes were significantly associated to caecal isolates (Appendix Table 8.7).

With isolates from the ileum, Scoary identified 41 genes linked with this site, excluding 50 hypothetical proteins. A wide variety of genes were found to be related to isolates from the ileum including a major exporter protein $(c h u A)$, an RNA polymerase subunit $($ rpoBC), an actin cross-linking toxin ( $\operatorname{vgrGl})$ and a tyrosine recombinase (xerH) (Table 5.2). The genes $\operatorname{vgrG}, x e r H, r p o B, c h u A$, fusA, $r p s L$ and $b c r$ were all found to be traitpositive in 12 of the 14 isolates from the ileum. All 41 genes were significantly related to presence in the ileum (Appendix Table 8.8).

Scoary found 178 genes linked to isolates extracted from the liver, excluding 173 hypothetical proteins. Genes related to isolates from the liver included: a heat shock protein $(h s p R)$, a transporter protein $(f e o A)$, an arsenical resistance protein $(a s r B)$ and a ribosomal protein methyltransferase ( $p r m A$ ) (Table 5.3). The genes acpS, arsB and arsC were found to be trait-positive in 12 of the 14 isolates, all yielding a p number of 0.013019. All 178 genes linked to the liver were found to be significant (Appendix Table 8.9). 
Table 5.2. Top genes found to be related to caeca isolates with the highest trait positive scores.

\begin{tabular}{|c|c|c|c|c|c|}
\hline Gene & Locus Tag & Annotation & $\begin{array}{l}C . \\
\text { jejun } \\
i \text { or } \\
C . \\
\text { coli } \\
\text { gene }\end{array}$ & $\begin{array}{l}\text { Trait } \\
\text { Positiv } \\
\text { e } \\
\text { Isolate } \\
\text { s }\end{array}$ & p Value \\
\hline leuS & $\mathrm{Cj} 1091 \mathrm{c}$ & Leucine tRNA ligase & $\begin{array}{c}C . \\
\text { jejun } \\
i\end{array}$ & 11 & 0.00869 \\
\hline $\operatorname{argS}$ & $\mathrm{Cj} 1175 \mathrm{c}$ & Arginine tRNA ligase & $\begin{array}{l}C . \\
\text { jejun } \\
i\end{array}$ & 11 & 0.017179 \\
\hline$m a c B$ & $\begin{array}{l}\text { ATE51_RS0 } \\
5905\end{array}$ & Macrolide export protein MacB & $\begin{array}{l}\text { C. } \\
\text { coli }\end{array}$ & 11 & 0.037322 \\
\hline gatA & Cj1059c & $\begin{array}{l}\text { Glutamyl-tRNA(Gln) } \\
\text { amidotransferase subunit A }\end{array}$ & $\begin{array}{l}C . \\
\text { jejun } \\
i \\
\end{array}$ & 11 & 0.040346 \\
\hline ompA & Cj0599 & Outer membrane protein $\mathrm{A}$ & $\begin{array}{l}\text { C. } \\
\text { jejun } \\
i\end{array}$ & 8 & 0.032929 \\
\hline pseC & Cj1294 & $\begin{array}{l}\text { UDP-4-amino-4,6-dideoxy-N- } \\
\text { acetyl-beta-L-altrosamine } \\
\text { transaminase }\end{array}$ & $\begin{array}{l}C . \\
\text { jejun } \\
i\end{array}$ & 7 & 0.04234 \\
\hline valS & $\mathrm{Cj} 0775 \mathrm{c}$ & Valine tRNA ligase & $\begin{array}{l}C . \\
\text { jejun } \\
i\end{array}$ & 5 & 0.012217 \\
\hline kefC & Cj1231 & $\begin{array}{l}\text { Glutathione-regulated potassium- } \\
\text { efflux system protein KefC }\end{array}$ & $\begin{array}{l}\text { C. } \\
\text { jejun } \\
i\end{array}$ & 5 & 0.012217 \\
\hline
\end{tabular}


Table 5.3. Top genes found to be related to ileum isolates with the highest trait positive scores

\begin{tabular}{|c|c|c|c|c|c|}
\hline Gene & Locus Tag & Annotation & \begin{tabular}{|l|}
$C$. \\
jejuni \\
or $C$. \\
coli \\
gene \\
\end{tabular} & $\begin{array}{l}\text { Trait } \\
\text { Positive } \\
\text { Isolates }\end{array}$ & p Value \\
\hline vgrGl & ATE51_RS07040 & Actin cross-linking toxin VgrG1 & $\begin{array}{c}C . \\
\text { coli }\end{array}$ & 12 & 0.006097 \\
\hline xerD & $\mathrm{Cj} 0863 \mathrm{c}$ & Tyrosine recombinase XerH & $\begin{array}{r}C . \\
\text { jejuni }\end{array}$ & 12 & 0.013019 \\
\hline rpoB & Cj0478 & $\begin{array}{l}\text { Bifunctional DNA-directed RNA } \\
\text { polymerase subunit beta-beta' }\end{array}$ & $\begin{array}{r}C . \\
\text { jejuni }\end{array}$ & 12 & 0.033572 \\
\hline fusA & $\mathrm{Cj} 0493$ & Elongation factor $\mathrm{G}$ & $\begin{array}{r}C . \\
\text { jejuni }\end{array}$ & 12 & 0.033572 \\
\hline$r p s L$ & Cj0491 & $30 \mathrm{~S}$ ribosomal protein $\mathrm{S} 12$ & $\begin{array}{r}C . \\
\text { jejuni }\end{array}$ & 12 & 0.033572 \\
\hline$b c r$ & ATE51_RS00200 & Bicyclomycin resistance protein & $\begin{array}{r}C . \\
\text { coli } \\
\end{array}$ & 12 & 0.033572 \\
\hline capA & Cj0628 & Lipoprotein & $\begin{array}{r}C . \\
\text { jejuni }\end{array}$ & 11 & 0.037139 \\
\hline chuA & $\mathrm{Cj} 1614$ & Outer membrance receptor & $\begin{array}{r}C . \\
\text { jejuni }\end{array}$ & 11 & 0.037139 \\
\hline rpmB & $\mathrm{Cj} 0450 \mathrm{c}$ & 50S ribosomal protein L28 & $\begin{array}{r}C . \\
\text { jejuni }\end{array}$ & 11 & 0.044913 \\
\hline metF & $\mathrm{Cj} 1202$ & $\begin{array}{l}5,10 \text {-methylenetetrahydrofolate } \\
\text { reductase }\end{array}$ & $\begin{array}{r}C . \\
\text { jejuni }\end{array}$ & 10 & 0.031294 \\
\hline
\end{tabular}

Table 5.4. Top genes found to be related to liver isolates with the highest trait positive scores

\begin{tabular}{|c|c|c|c|c|c|}
\hline Gene & Locus Tag & Annotation & $\begin{array}{l}C . \text { jejuni } \\
\text { or } C . \text { coli } \\
\text { gene }\end{array}$ & $\begin{array}{l}\text { Trait } \\
\text { Positive } \\
\text { Isolates }\end{array}$ & p value \\
\hline acpS & Cj1409 & $\begin{array}{l}\text { Holo-[acyl-carrier-protein] } \\
\text { synthase }\end{array}$ & C. jejuni & 12 & 0.013019 \\
\hline $\operatorname{ars} B$ & $\mathrm{Cj} 1187 \mathrm{c}$ & Arsenical-resistance protein Acr3 & C. jejuni & 12 & 0.013019 \\
\hline $\operatorname{arsC}$ & Cj0717 & Glutaredoxin arsenate reductase & C. jejuni & 12 & 0.013019 \\
\hline$f e o A$ & Cj1397 & $\begin{array}{l}\text { Putative } \mathrm{Fe}(2) \text { transport protein } \\
\text { A }\end{array}$ & C. jejuni & 11 & 0.005736 \\
\hline galE & $\mathrm{Cj} 1131 \mathrm{c}$ & UDP-glucose 4-epimerase & C. jejuni & 11 & 0.044913 \\
\hline$\overline{p r m A}$ & $\mathrm{Cj} 1117 \mathrm{c}$ & $\begin{array}{l}\text { Ribosomal protein L11 } \\
\text { methyltransferase }\end{array}$ & C. jejuni & 10 & 0.031294 \\
\hline purU & $\mathrm{Cj} 0790$ & $\begin{array}{l}\text { Formyltetrahydrofolate } \\
\text { deformylase }\end{array}$ & C. jejuni & 10 & 0.031294 \\
\hline hspR & $\mathrm{Cj} 1230$ & Putative heat shock protein HspR & C. jejuni & 10 & 0.031294 \\
\hline ribA & Cj0996 & GTP cyclohydrolase-2 & C. jejuni & 10 & 0.031294 \\
\hline$f a b D$ & Cj0116 & $\begin{array}{l}\text { Malonyl CoA-acyl carrier protein } \\
\text { transacylase }\end{array}$ & C. jejuni & 10 & 0.031294 \\
\hline
\end{tabular}




\subsubsection{Invasive capability of Campylobacter strains isolated from different sections of the chicken gut and liver from naturally infected chickens}

Fourty two Campylobacter strains were used in total with 14 strains each from the caeca, ileum and liver. The invasive capability of these strains was measured using a gentamicin protection assay on two cell lines; one human (Caco-2) and one chicken (8E11) (Figure 5.3). The majority of Campylobacter strains (12/14 from caeca, 13/14 from ileum and 14/14 from liver) could effectively invade both cell lines with a slight trend of higher invasion in the chicken 8E11 cells. However, there was no significant difference between the two cell lines (Figure 5.3a).

Comparison of the strains isolated from the caeca and ileum found no significance between levels of invasion (Figure 5.3bc), however strains isolated from the liver demonstrated a higher invasive capability in the chicken 8E11 cells compared to the Caco-2 ones with a significant $(\mathrm{p} \leq 0.05)$ difference between the two groups. All liver isolates were able to invade into both cell lines (Figure 5.3d).

Sub-group analysis of Campylobacter isolates from caeca, ileum or liver produced important significant differences $(\mathrm{p}<0.05)$ (Figure 5.3ef). In the chicken $8 \mathrm{E} 11$ cells, the liver isolates had the highest overall invasive capability with significant differences seen when comparing the liver and caeca $(\mathrm{p} \leq 0.001)$ or liver and ileum $(\mathrm{p} \leq 0.001)$ (Figure 5.1e). A similar pattern was observed in the human Caco-2 cells with strains isolated from the liver again demonstrating the highest overall invasion ability with a significant difference seen when comparing them to the caecal isolates $(\mathrm{p} \leq 0.01)$. There was no significant difference between strains from liver and ileum (Figure 5.3f).

Further sub-group analysis of Campylobacter from caeca, ileum and liver from the same bird confirmed that invasion was less dependent on 'bird' and more dependent on anatomical site within it (Figure 5.4) and the genes identified in the individual strains (Tables 5.2 - 5.4). In the $8 \mathrm{E} 11$ cell line, there was a much greater level of invasion by the liver isolates compared to those from the caeca and ileum from the same birds (Figure 5.4a). One of the birds had strains of Campylobacter which displayed no invasion in the caeca and ileum, however; the strain isolated from the liver was very highly invasive, producing the joint highest invasive response recorded (Figure 5.4a). Invasion responses in Caco- 2 cells displayed a similar pattern as in the $8 \mathrm{E} 11$ cells 
except for isolates from one bird in which the strain isolated from the caeca produced the highest invasive response recorded. The strain from the liver was also high, whereas that from the ileum was much less invasive (Figure 5.4b). Another strain isolated from caeca was unable to invade into the Caco-2 cells; whereas, ones from the ileum and liver of the same bird produced very high invasive responses (Figure 5.4b).

a

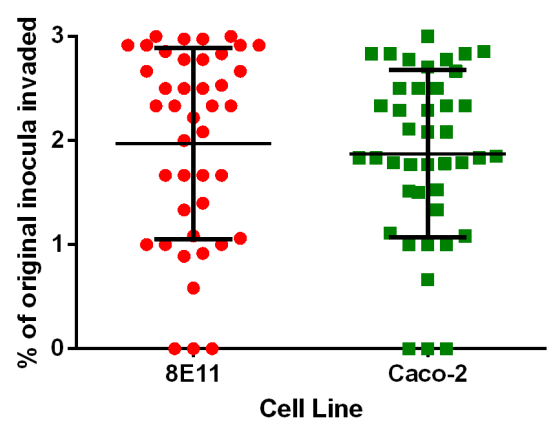

C
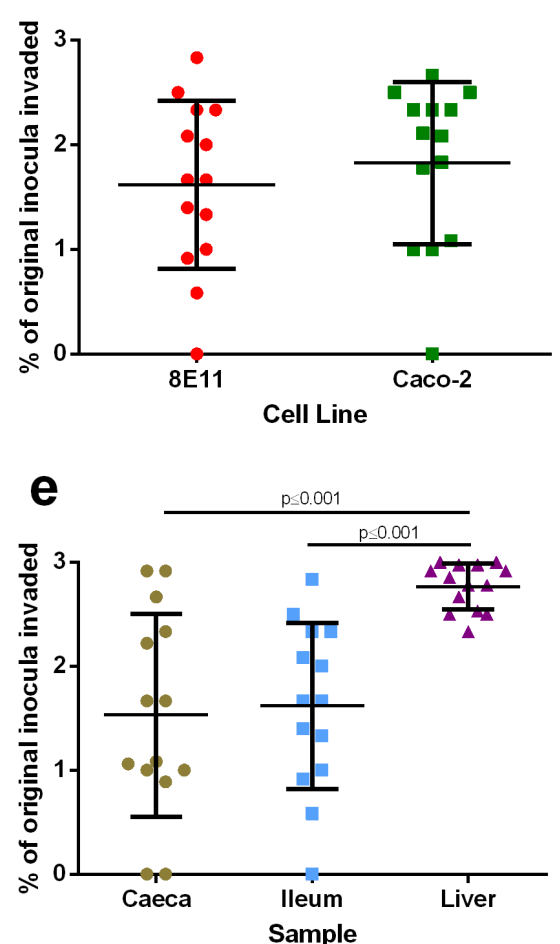
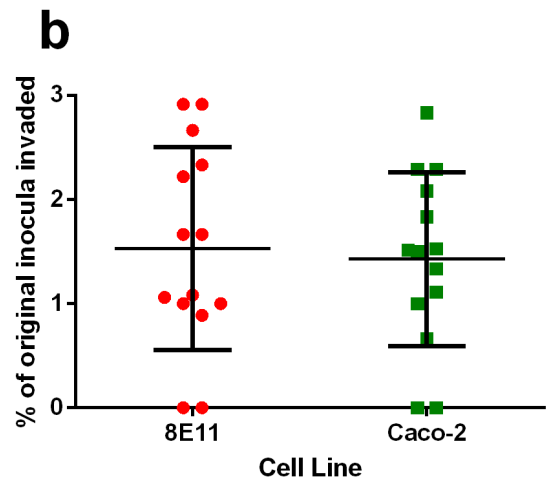

d
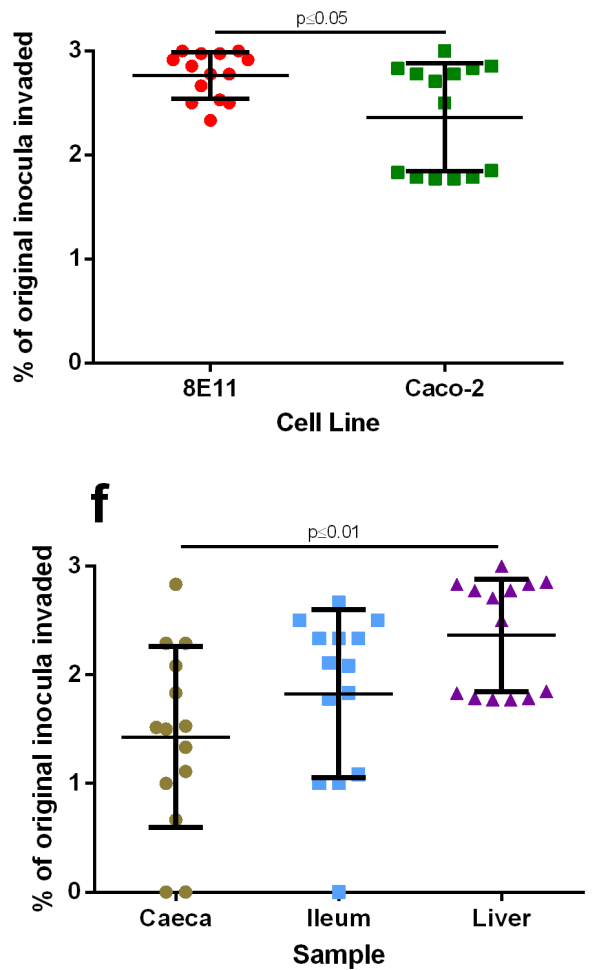

Figure 5.3. Gentamicin protection assay of 42 Campylobacter strains from caeca, ileum and liver in 8E11 and Caco-2 cells (a), with comparison of strains from the (b) caeca, (c), ileum and (d) liver in both cell lines, with the breakdown of invasive responses by 
group in (e) 8E11 and (f) Caco-2. Results are also expressed as mean $+/-$ SD of all strains measured. Differences were considered significant if $\mathrm{p} \leq 0.05$.

a

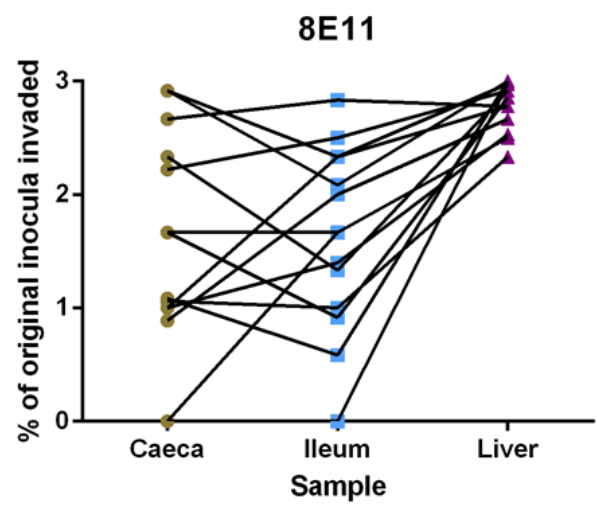

b

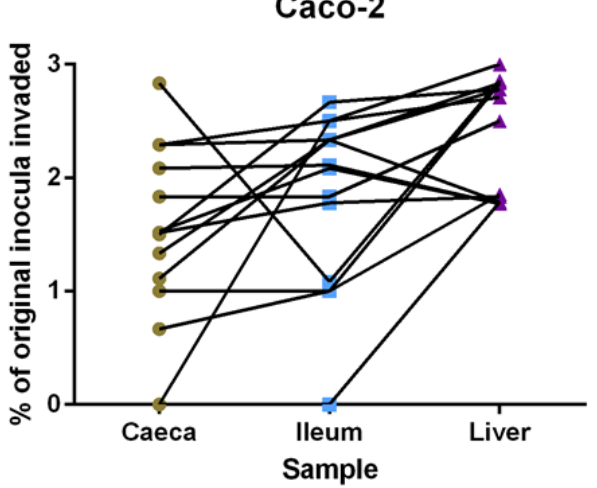

\begin{tabular}{|c|c|c|c|c|c|c|c|}
\hline \multirow[t]{2}{*}{ Bird } & \multicolumn{3}{|c|}{$\begin{array}{l}\% \text { of original inocula invaded into } 8 \mathrm{E} 11 \\
\text { cells }\end{array}$} & \multirow[t]{2}{*}{ Bird } & \multicolumn{3}{|c|}{$\begin{array}{c}\% \text { of original inocula invaded into Caco-2 } \\
\text { cells }\end{array}$} \\
\hline & Caeca & Ileum & Liver & & Caeca & Ileum & Liver \\
\hline 7 & 2.92 & 2.08 & 3.00 & 7 & 2.29 & 2.50 & 3.00 \\
\hline 8 & 0.89 & 2.00 & 2.67 & 8 & 1.33 & 2.33 & 2.83 \\
\hline 11 & 1.06 & 1.00 & 2.33 & 11 & 1.52 & 1.78 & 1.83 \\
\hline 12 & 1.67 & 0.92 & 2.86 & 12 & 2.83 & 1.08 & 2.86 \\
\hline 14 & 2.92 & 2.33 & 2.98 & 14 & 2.29 & 2.33 & 1.79 \\
\hline 15 & 1.67 & 1.67 & 2.50 & 15 & 2.08 & 2.11 & 1.77 \\
\hline 18 & 0.00 & 0.00 & 2.98 & 18 & 0.00 & 0.00 & 1.79 \\
\hline 20 & 0.00 & 1.67 & 2.50 & 20 & 0.00 & 2.50 & 2.71 \\
\hline 23 & 1.08 & 0.58 & 3.00 & 23 & 0.67 & 1.00 & 2.83 \\
\hline 24 & 1.00 & 1.40 & 2.53 & 24 & 1.00 & 1.00 & 1.85 \\
\hline 25 & 2.22 & 2.50 & 2.92 & 25 & 1.53 & 2.08 & 1.77 \\
\hline 26 & 2.67 & 2.83 & 2.78 & 26 & 1.50 & 2.67 & 2.78 \\
\hline 28 & 1.00 & 2.33 & 2.78 & 28 & 1.11 & 2.33 & 2.78 \\
\hline 29 & 2.33 & 1.33 & 2.92 & 29 & 1.83 & 1.83 & 2.50 \\
\hline
\end{tabular}

Figure 5.4. Gentamicin protection assay results with strains isolated from the same bird joined across each anatomical location (a) 8E11 and (b) Caco-2 cells. Individual results are presented in the table. 


\subsubsection{Analysis of the invasive capability of Campylobacter strains in vitro}

Gentamicin protection assays were used to measure the invasive capabilities of the six Campylobacter strains used during in vivo infection studies. The strains were added to three epithelail cell lines: the HT-29 and Caco-2 human lines and 8E11 chicken line. All strains were able to invade each cell line to a similar degree with $0.9-1.8 \%$ of original inocula of each of the six strains being invasive (Figure 5.5a), and no differences seen between the cell lines. L4, a strain isolated from the liver of a naturally infected chicken produced the largest invasion response in the 8E11 and Caco-3 cell lines (Figure 5.5b), while a typed strain, CJ12662, was the least invasive. Each strain demonstrated consistent responses between cell lines, displaying a similar level of invasion in each (Figure 5.5b). Both strains isolated from the caeca, C8 and C15, had similar levels of invasion in all cell lines. No significance difference was found between cell lines or Campylobacter strain. All six strains were combined to infect the cells, which resulted in invasion rates of around 1.4-1.6\% (Figure 5.5, grey points).

a

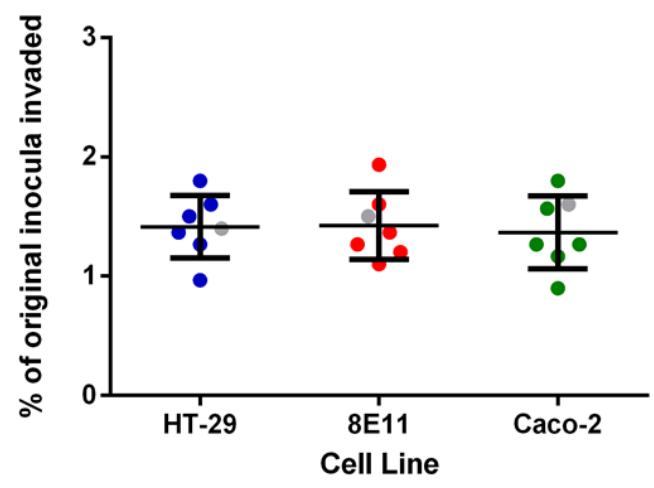

b

\begin{tabular}{|l|r|r|r|}
\hline \multirow{2}{*}{ Strain } & \multicolumn{4}{|c|}{ \% of original inocula invaded } \\
\cline { 2 - 4 } & HT-29 & \multicolumn{1}{c|}{ 8E11 } & Caco-2 \\
\hline C8 & 1.50 & 1.27 & 1.57 \\
\hline C15 & 1.37 & 1.10 & 1.17 \\
\hline G4 & 1.80 & 1.60 & 1.27 \\
\hline L4 & 1.60 & 1.93 & 1.80 \\
\hline Cj12662 & 0.97 & 1.20 & 0.90 \\
\hline Cj13126 & 1.27 & 1.37 & 1.27 \\
\hline Combined & 1.40 & 1.50 & 1.60 \\
\hline
\end{tabular}

Figure 5.5. Gentamicin protection assay performed on three cell lines, HT-29, 8E11 and Caco-2 displaying overall invasive capability of the strains with invasion of all six strains combined included in grey (a). The individual results are presented in the table through each cell line (b). Results are also expressed as mean +/- SD of all strains measured. Differences were considered significant if $\mathrm{p} \leq 0.05$. 


\subsubsection{In vitro expression of pro-inflammatory cytokines in human and chicken cell}

lines

RNA was isolated from human and chicken epithelial cells after being treated with the six Campylobacter strains for 24 hours. The cDNA generated from the total RNA was subsequently used for quantitative PCR to measure pro- and anti- inflammatory responses (including IL-8 as a marker for the pro- inflammatory response and IL-10 as the marker for the anti-inflammatory response). Results of the pro-inflammatory response displayed slightly higher expression of IL-8/CXCLi2 compared to CXCLi1. However, there was no significant difference between expression of IL-8/CXCLi1/2 in each cell line (Figure 5.6a). C. jejuni strain CJ12662 caused the lowest overall level of expression in every cell line tested. In contrast, L4, a strain isolated from the liver, caused the highest overall expression of IL-8, in the HT-29 and Caco-2, and CXCLi2 in the $8 \mathrm{E} 11$ cells (Figure 5.6b). C. jejuni strain CJ13126 produced the highest expression of CXCLi1 in the 8E11 cells (Figure 5.6b). The two strains isolated from the caeca of a chicken, C8 and C15, invoked similar patterns of expression in the cell lines with that in HT-29 and Caco-2, yielding almost identical results for each isolate. When the six strains were combined and used to infect the cells lines, the expression of IL-8 and CXCLi1/2 were observed to be higher than the average expression of the individual strains (Figure 5.6, grey points).

\section{a}

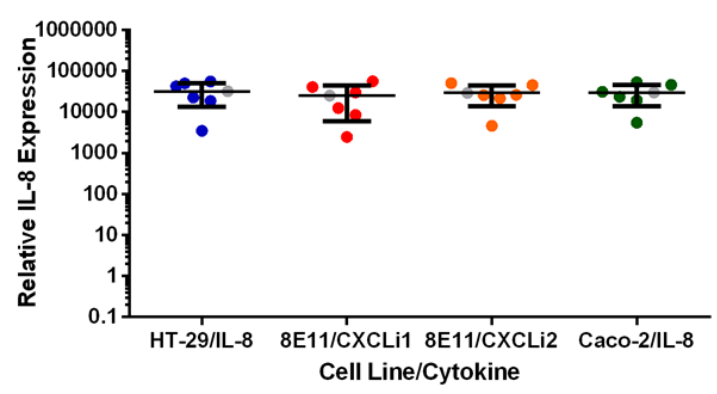

b

\begin{tabular}{|l|r|r|r|r|}
\hline \multirow{3}{*}{ Strain } & \multicolumn{5}{|c|}{ Relative expression of IL-8/CXCL1/2 } \\
\cline { 2 - 6 } & HT-29/L-8 & $\begin{array}{c}\text { 8E11/ } \\
\text { CXCLi1 }\end{array}$ & \multicolumn{1}{c|}{$\begin{array}{c}\text { 8E11/ } \\
\text { CXCLi2 }\end{array}$} & Caco-2/L-8 \\
\hline C8 & 18563.15 & 8485.48 & 26185.08 & 19475.65 \\
\hline C15 & 49521.36 & 29854.21 & 25846.11 & 45863.15 \\
\hline G4 & 22548.33 & 12432.15 & 21685.78 & 23485.12 \\
\hline L4 & 55486.69 & 40785.36 & 50486.82 & 53118.33 \\
\hline Cj12662 & 3465.66 & 2466.11 & 4586.19 & 5486.66 \\
\hline Cj13126 & 42157.22 & 56183.04 & 45156.68 & 31254.13 \\
\hline Combined & 31823.07 & 25145.39 & 28967.11 & 29713.51 \\
\hline
\end{tabular}

Figure 5.6. Expression of IL-8 and CXCLi1/2 in the three cell lines tested; HT-29, $8 \mathrm{E} 11$ and Caco-2 with all six strains combined seen in grey (a), Theindividual results are presented within the table (b). Results are also expressed as mean $+/-$ SD of all strains measured. Differences were considered significant if $\mathrm{p} \leq 0.05$. 


\subsubsection{In vitro expression of anti-inflammatory IL-10 in human and chicken cell}

lines

Results for the anti-inflammatory marker IL-10 showed that there was no significant difference in its expression by different cell lines (Figure 5.7a). Similar to expression of IL-8; Campylobacter strain CJ12662 produced the lowest level of expression in all three cell lines, with the strain isolated from the liver of an infected chicken, L4, invoking the highest anti-inflammatory response in all lines (Figure 5.7b). Expression invoked by the two strains from caeca were similar, and greater than that invoked by the strain isolated from the ileum (Figure 5.7b). When six strains were used together to infect cells lines expression of IL-10 was observed to express at the average rate of expression of the individual strains (Figure 5.7, grey points).

a

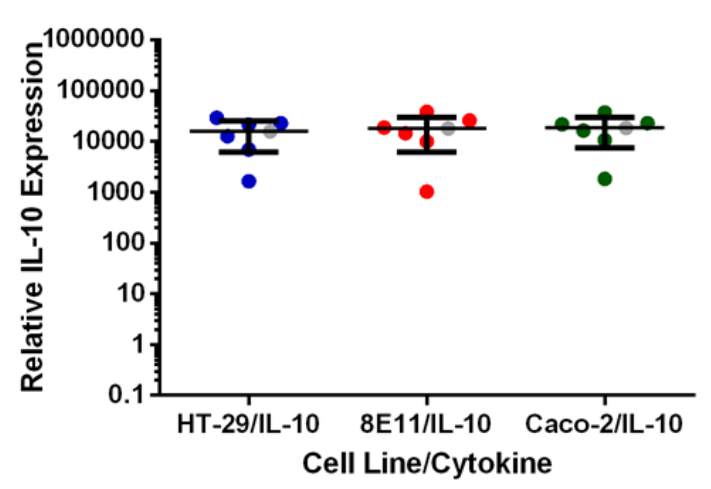

b

\begin{tabular}{|l|r|r|r|}
\hline \multirow{2}{*}{ Strain } & \multicolumn{3}{|c|}{ Relative expression of IL-10 } \\
\cline { 2 - 4 } & HT-29/L-10 & 8E11/IL-10 & Caco-2/IL-10 \\
\hline C8 & 12681.29 & 18709.34 & 16285.35 \\
\hline C15 & 22837.39 & 14373.77 & 21652.33 \\
\hline G4 & 6875.54 & 9971.307 & 10658.36 \\
\hline L4 & 28954.98 & 37995 & 36842.88 \\
\hline Cj12662 & 1645.987 & 1028.644 & 1832.23 \\
\hline Cj13126 & 21429.42 & 25702.42 & 22894.12 \\
\hline Combined & 15812.43 & 17955.41 & 18310.88 \\
\hline
\end{tabular}

Figure 5.7. Expression of IL-10 in the three cell lines tested; HT-29, $8 \mathrm{E} 11$ and Caco-2 with all six strains combined shown in grey(a). The individual results are presented in the table(b). Invasion with all six strains combined can be seen in grey. Results are also expressed as mean +/- SD of all strains measured. Differences were considered significant if $\mathrm{p} \leq 0.05$. 


\subsubsection{Expression of immune biomarkers in caecal tonsils extracted from Campylobacter-infected chickens}

The in vivo chicken immune response to Campylobacter was measured in the caecal tonsils extracted at post-mortem (PM) of uninfected and infected birds. The two groups of chickens were kept in separate chicken houses and not moved (Figure 5.8). Cytokines responses measured included $\mathrm{T}_{\mathrm{h}} 1 / \mathrm{T}_{\mathrm{h}} 2$ (Figure 5.8bce), $\mathrm{T}_{\mathrm{h}} 17$ (Figure 5.8h), $\mathrm{T}_{\mathrm{h}} 22$ (Figure 5.8df) and cytokines involed in innate immunity (Figure 5.8ag).

Cytokines involved in the $T_{h} 1 / T_{h} 2$ pathway were found overall to increase significantly in birds infected with Campylobacter compared to uninfected ones (Figure 5.8 a-e). The pro-inflammatory cytokine, IL-8 (Figure 5.8a) and IL-10 (Figure 5.8b), an antiinflammatory effector cytokine of Th2 response, were significantly higher $(\mathrm{p} \leq 0.0001)$ in the infected birds. IL-18, a $\mathrm{T}_{\mathrm{h}} 1$ cytokine involved in cell-mediated immunity expressed significantly more IFN $\gamma$ in the infected birds compared to the uninfected ones $\left(\mathrm{p} \leq 0.000\right.$; Figure 5.8c). A measurement of $T N F \alpha, a T_{h} 22$ cytokine involved in systemic inflammation in chickens, and IFN $\gamma$, a cytokine important to both the innate and adaptive immune response, secreted by $T_{h} 1$ cells, showed significantly higher expression in the infected birds compared to the controls ( $\mathrm{p} \leq 0.000$; Figure 5.8de).

An early cytokine, which modulated activity and is involved in all $\mathrm{T}_{\mathrm{h}}$ responses, IL-6, was measured; with the uninfected group showing significantly $(\mathrm{p} \leq 0.0001)$ lower levels compared to the infected group (Figure 5.8f). Levels of IL-1 $\beta$, a mediator of the inflammatory response and promoter of $\mathrm{T}_{\mathrm{h}} 17$, and IL-17, a pro-inflammatory cytokine secreted by $\mathrm{T}_{\mathrm{h}} 17$ cells were measured and were expressed significantly more in infected birds ( $\mathrm{p} \leq 0.0001$; Figure 5.8gh).

The expression of the immune biomarkers was then compared to the Campylobacter counts of the caeca and ileum (Figure 5.9 and 5.10). Within the caeca of infected chickens there appeared to be no correlation between the number of Campylobacter and the expression of the $T_{h} 1 / T_{h} 2, T_{h} 17$ and $T_{h} 22$ cytokines expressed from the caecal tonsils (Figure 5.9). Campylobacter counts from the ileum displayed a similar pattern with no significant correlation ( $p>0.05$ ) seen between expression from the immune markers in the caecal tonsils to the number of Campylobacter in the ileum (Figure 5.10). 

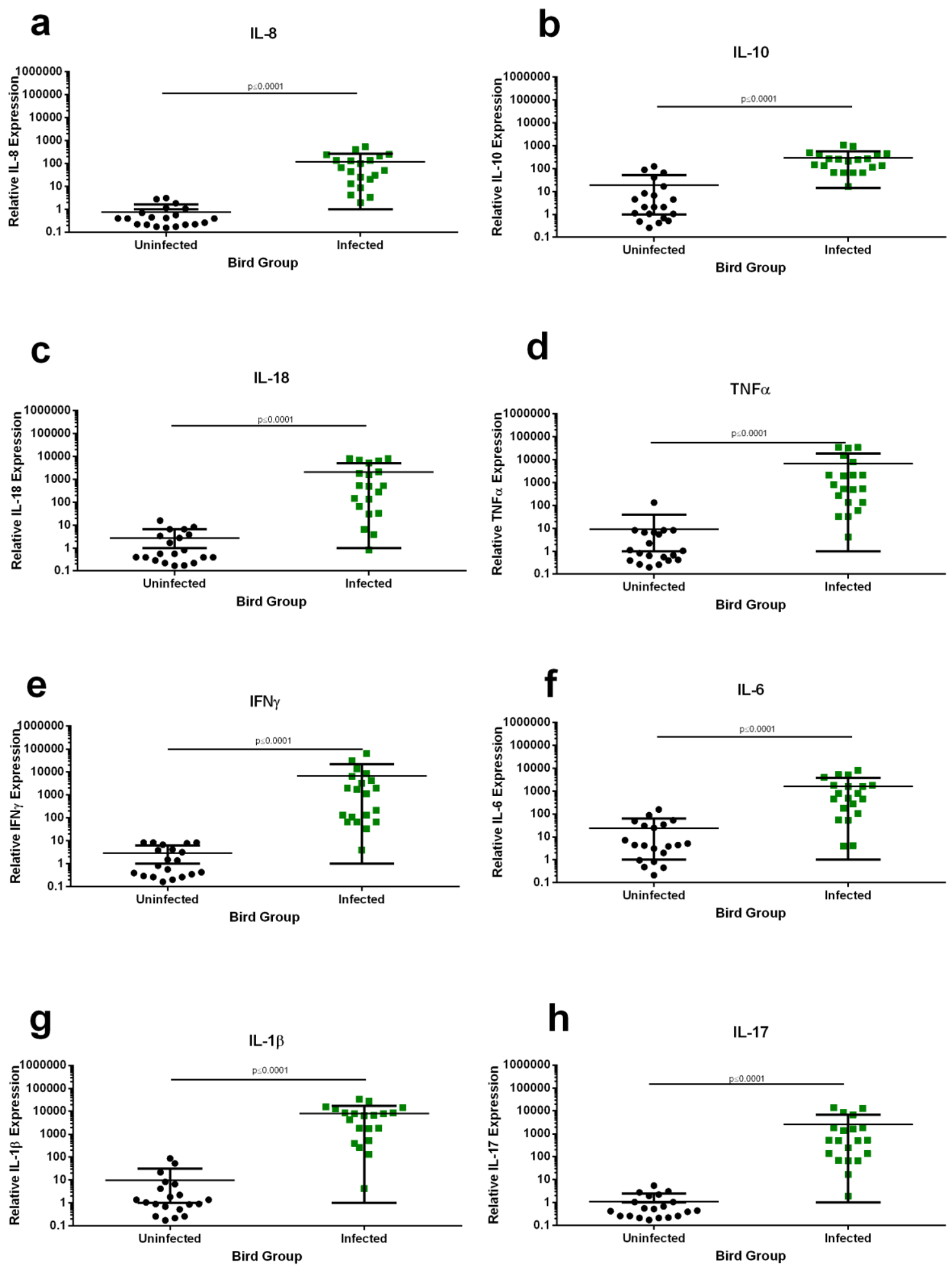

Figure 5. 8 Expression of a variety of $T_{h} 1 / T_{h} 2, T_{h} 17$ and $T_{h} 22$ (f,g,h) markers in caecal tonsils extracted from uninfected chicken, chickens that were infected and kept in chicken sheds and chickens who were infected and put through the industry production chain including being handled, transportation and gas/stun killed. Immune markers measured were (a) IL-8, (b) IL-10, (c) IL-18, (d) TNF $\alpha$, (e) IFN $\gamma$, (f) IL-6, (g) IL-1 $\beta$ and (h) IL-17. Results are also expressed as mean $+/$ - SD of all strains measured. Differences were considered significant if $\mathrm{p} \leq 0.05$. 

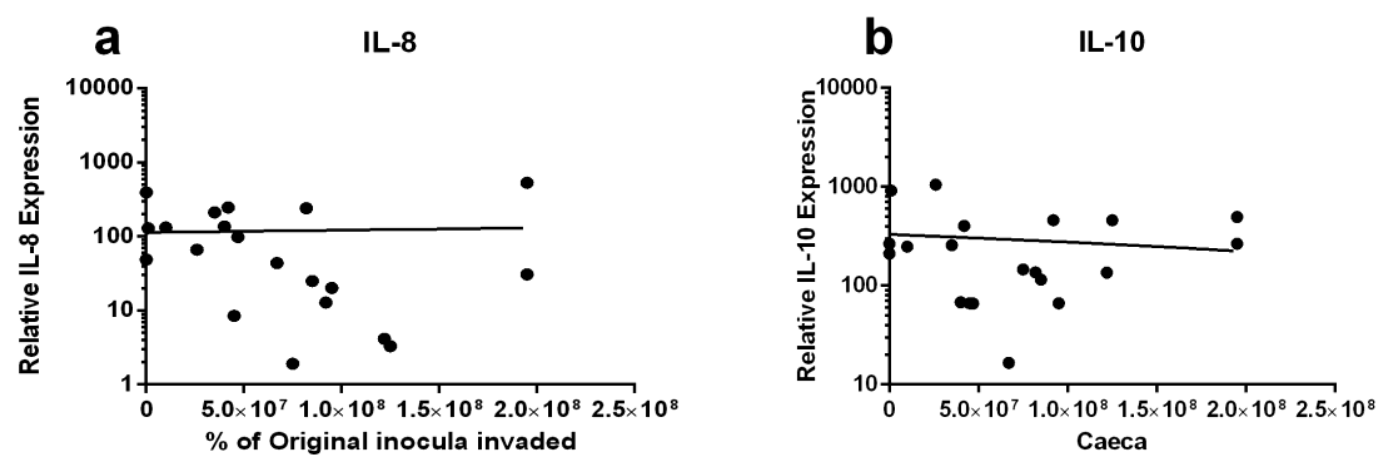

C
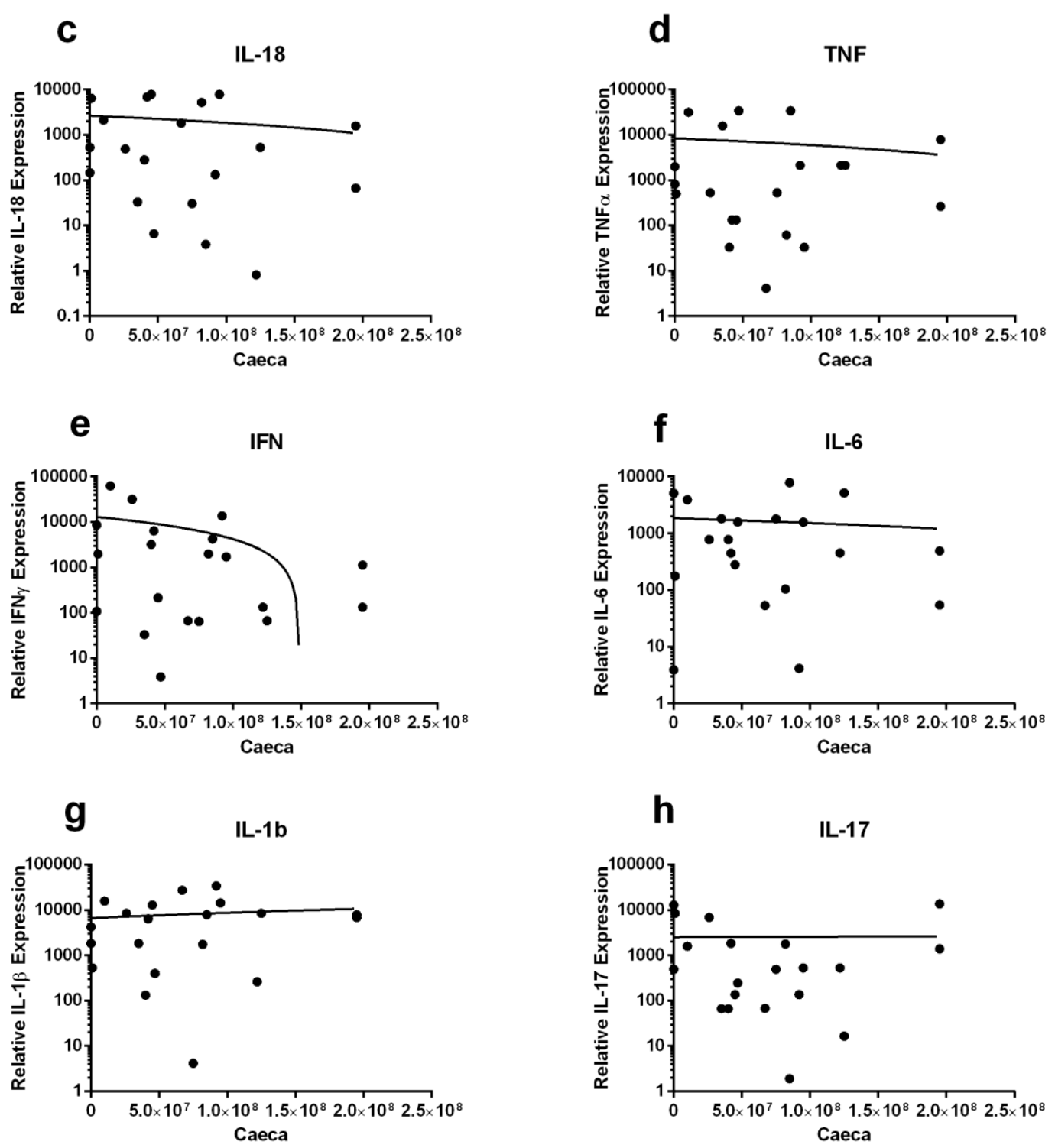

Figure 5.9. Correlations of Campylobacter counts in the caeca compared to expression of cytokines. Immune markers measured were (a) IL-8, (b) IL-10, (c) IL-18, (d) TNF $\alpha$, (e) IFN $\gamma$, (f) IL-6, (g) IL-1 $\beta$ and (h) IL-17. Results are also expressed as mean +/- SD of all strains measured. Differences were considered significant if $\mathrm{p} \leq 0.05$. 
a

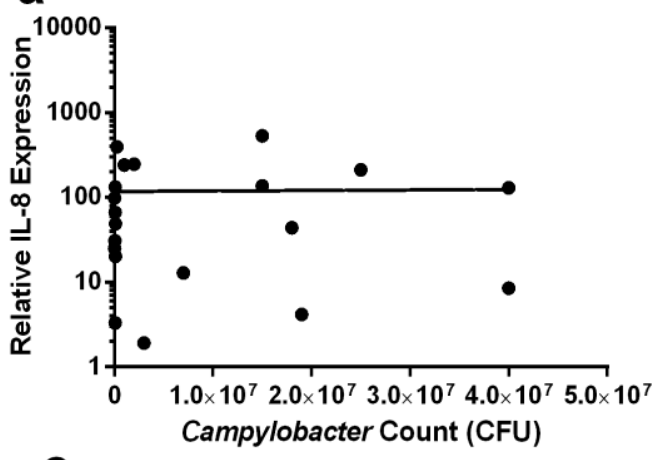

C

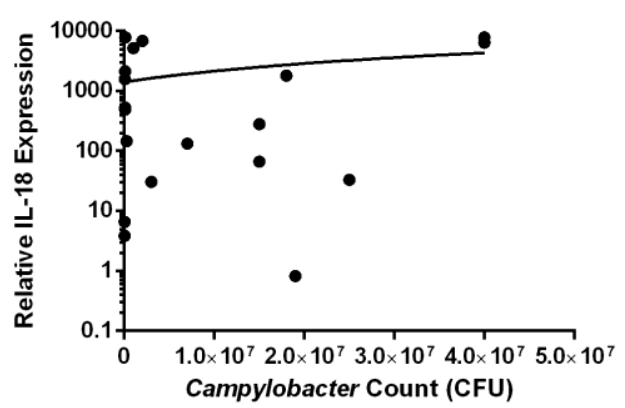

e

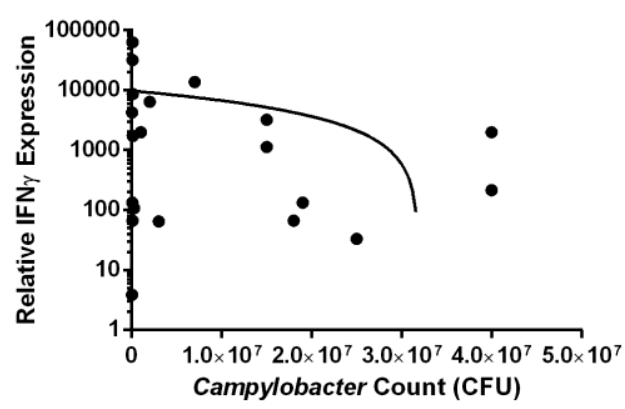

g

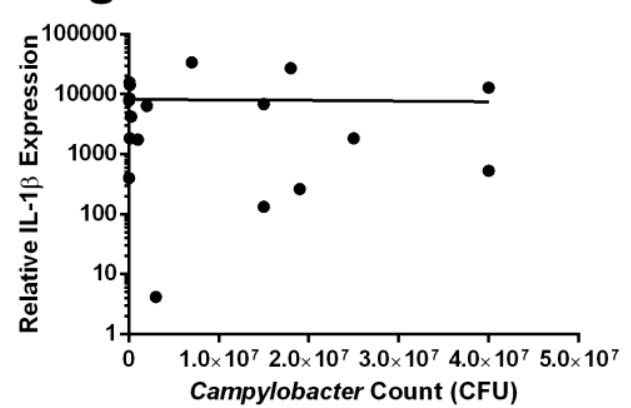

b
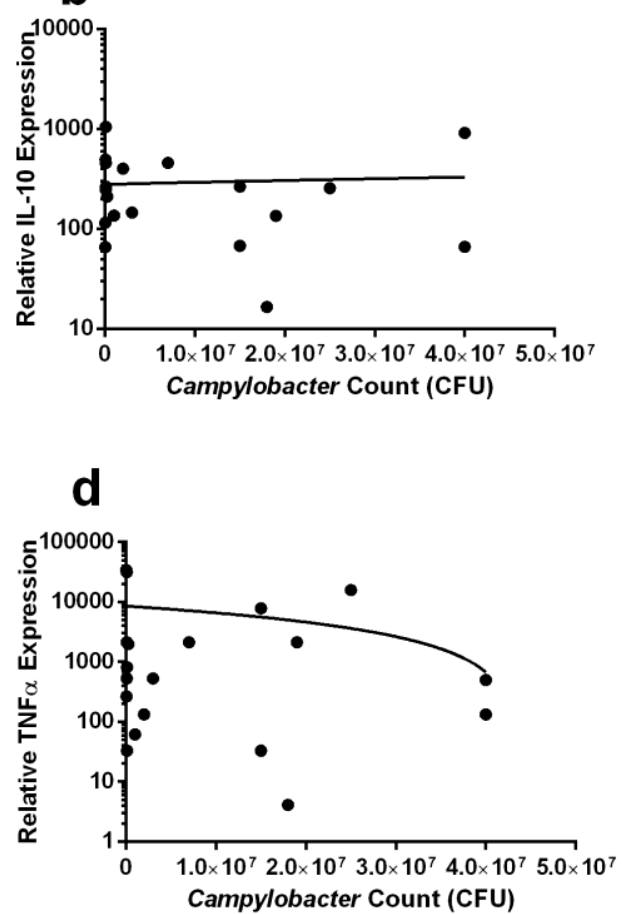

$\mathbf{f}$

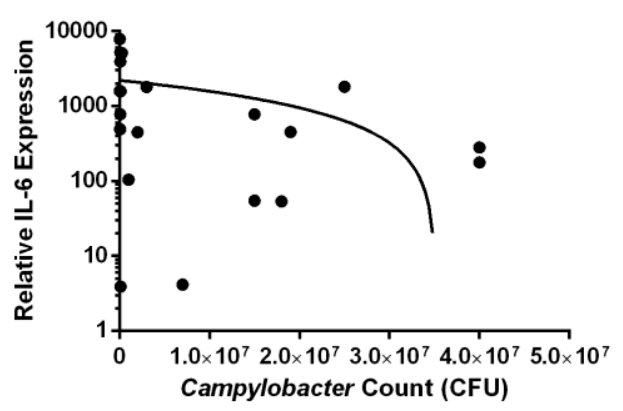

h

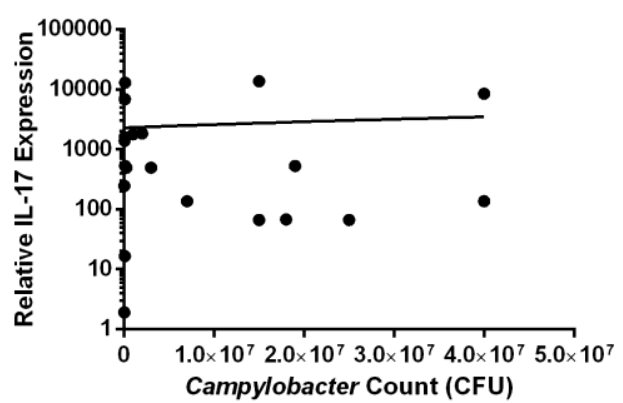

Figure 5.10. Correlations of Campylobacter counts in the ileum compared to expression of cytokines. Immune markers measured were (a) IL-8, (b) IL-10, (c) IL-18, (d) TNF $\alpha$, (e) IFN $\gamma$, (f) IL-6, (g) IL-1 $\beta$ and (h) IL-17. Results are also expressed as mean +/- SD of all strains measured. Differences were considered significant if $\mathrm{p} \leq 0.05$. 


\subsection{Discussion}

This chapter focuses on two sets of data, one set related to Campylobacter strains from naturally infected chickens. This set identifies genomic markers in Campylobacter associated with presence in the caeca, ileum or liver. The second set of data relates to caecal tonsils extracted from experimentally infected chickens to measure host immune responses to assess potential immune biomarkers that define the Campylobacter infection response in chickens.

Fourteen naturally infected birds had Campylobacter in the caeca, ileum and liver and these became a collection of 42 'bird-matched' strains and were used to investigate potential differences isolates from the caeca, ileum and liver genomically and phenotypically. A pan-genome of all 42 isolates was created but two (C20 and C26) were of a poor sequence quality and thus were omitted. The resultant pan-genome contained 10,446 genes, with a very small core genome of 226 genes and 10,217 accessory ones. The small size of the core genome could be due to both $C$. jejuni and $C$. coli being present in the pan-genome, as previous studies have shown that no clear plateau of genome size occurs and more diverse isolates can cause some genes to be shared (Méric et al., 2014). Work by van Tonder et al., (2014) observed that the number of isolates can affect measured core genome size (van Tonder et al., 2014). Roary cut off of $95 \%$ for identity with blastp could also impact on the genome size and less stringent cut offs could alter the genome sizes (Page et al., 2015).

The maximum likelihood tree constructed from the pan genome displayed no clear grouping of isolates from any particular birds which demonstrates the diversity of the dataset. However, ileum and liver isolates from birds 7 and 11 were shown to cluster tightly together which could indicate that they were able to persist in both environments. Analysis of the pan-genome identified only two known virulence genes, pebAl and pldA, present in the core genome which could be due to its small size as discussed earlier. The two genes are both adhesion-linked genes with pebAl being shown to be interact with epithelial cell since mutations in it causes a reduction in interactions with epithelial cells and reduction of colonisation (Pei et al., 1998). The gene, pldA, which encodes for a phospholipase could be very useful for Campylobacter as the protein aids the bacteria's ability to invade into chickens as pldA deficient mutants were found to invade and colonize chickens at an impaired rate (Ziprin et al., 2001). 
Scoary analysis was able to identity genes linked solely to the caeca, the ileum and the liver, with isolates from the liver found to have the most associated genes (178 genes). The caeca isolates were found to have 60 genes linked to this trait including, macA, a macrolide export protein which makes up part of the MacAB multi-drug efflux system. This is an important protein in pathogenicity and host interactions which detects $40 \%$ aerobiosis and secreted proteases, such as HtrA, which are more abundant at lower oxygen concentration (Guccione et al., 2017). This protein could help contribute to the survival of Campylobacter in the caeca and allow the bacteria to proliferate in the gut environment. Another gene found linked to caecal isolates was ompA, a multi-functional outer membrane protein which plays a role in Campylobacter pathogenesis and may play a role in the ability to invade and has been seen to be upregulated during in vivo growth (Rathbun and Thompson, 2009; Stintzi et al., 2005).

Scoary identified 41 genes linked to isolates taken from the ileum including capA, an autotransporter protein which mediates association with epithelial cells and colonization of the chicken gut (Ashgar et al., 2007) . The liver isolates were found to have 178 related genes, among which were two arsenic resistance genes, $\operatorname{ars} B$ and $\operatorname{ars} C$, which could be related to arsenical compounds which have been used in the feed of poultry for pigmentation, growth promotion and weight gain and thus these genes may help survival in the blood of Campylobacter strains to leave the gut and enter the liver (Noormohamed and Fakhr, 2013). Another gene, feoA which encodes for an iron transporter and has been found to have a major role in iron acquisition, gut colonisation and survival in the gut and blood and so may help survival and proliferation in the liver (Naikare et al., 2006). The genes related to liver isolates could make for potential PCR biomarkers in Campylobacter strains for invasiveness as isolates from the liver where also found to be the most invasive.

The 42 Campylobacter strains from 14 birds which had Campylobacter in the caeca, ileum and liver were further investigated for virulence in an invasion assay on one human (Caco-2) and one chicken (8E11) cell line. In both cell lines, the Campylobacter strains isolated from the liver invaded the cells at a much higher rate compared to strains isolated from the caeca and the ileum in the 8E11 cells, this supports reported data that Campylobacter getting into the liver requires a high level of invasion (Jennings et al., 2011; Vaezirad et al., 2016; Williams et al., 2013). Three strains, one strain from the ileum and two from caeca, were unable to invade into either cell line. 
Campylobacter has variable invasion potential into cells, and is dependent on strain type. For example, the reference strain of NCTC11168 can invade cells at low levels of $1 \%$, other strains have been shown to invade between 1 and $5 \%$ of the original population (Friis et al., 2009; Koolman et al., 2016). The choice of cell line can also have an impact on the invasion potential of a strain. Caco- 2 cells were used during this experiment due to the vast number of studies using these cells when investigating the effect of infection with Campylobacter and for correlation from results obtained in vivo (Fonseca et al., 2013; Friis et al., 2009; Hanel et al., 2004; Man et al., 2010).

Of the 42 strains tested on the Caco-2 and 8E11 cells four were chosen to be used to infect chickens in the in vivo study (two strains from the caeca, one from the ileum and one from the liver). In addition, two strains; CJ13126 and CJ12662, previously used for in vivo infection studies (Friis et al., 2005; Mills et al., 2012), were also chosen to be used alongside the four isolated strains to be used as a cocktail to infect chickens. These six strains were also subjected to in vitro analysis on three cell lines, two human cell lines (HT-29 and Caco-2) and one chicken cell line (8E11). Both, invasiveness into each cell line and the inflammatory response was measured. The individual ability of the six strains to invade each cell line was very consistent with $0.9-2 \%$ of original inocula shown to invade the cells (Figure 5.5) and no significant differences observed between the strains tested. These results are consistent with invasive responses observed by others who used several different strains (Friis et al., 2005; Mills et al., 2012). CJ12662 produced the lowest levels of invasion in all cell lines tested, despite being reported previously to be invasive (Humphrey et al., 2015). To investigate synergy, the six strains were combined and were able to invade effectively and cause expression of pro- and anti- inflammatory cytokines in all three cell lines with the level of invasion shown to be between 1.4-1.6\%. Expression of IL-8/CXCli1/2 was observed to be higher than the average expression of the strains individually. No synergism between strains was observed however it would be interesting to speculate whether growth and invasion in all six strains remained consistent-this was not measured here.

Analysis of pro- and anti- inflammatory responses in the human and chicken cells in response to the six strains in vitro yielded similar results across all three cell lines with the cells being able to elicit a response to each Campylobacter strain, with no significant differences between cell lines. Interestingly, the characterised Campylobacter strain, CJ12662, was observed to elicit the lowest response in each cell line and was also seen 
to be the weakest invader into the cells. In contrast, the Campylobacter strain isolated from the liver of an infected chicken, L4, was the strongest invader into the cells and produced the highest expression of the IL-8/CXCLi1/CXCLi2 and IL-10. These results support previous research, by other and within Chapter 4 that a higher pro-inflammatory response is linked to higher invasion rate (John et al., 2017; Larson et al., 2008). In addition, the fact that the Campylobacter strain with the highest response was from the liver of infected chickens gives weight to the hypothesis that Campylobacter must be highly invasive to leave the gut and enter the liver.

The cocktail of these six strains was then used to infect chickens in vivo using a novel seeding method (Sandilands et al., 2018). The 'infected' and 'untreated' group of birds, were housed separately to minimise risk of cross-contamination with Campylobacter. RNA extracted was used to assess expression of the immune markers; IL-6, IL-8, IL-10, IL-1 $\beta$, TNF $\alpha$ and IFN $\gamma$. Levels of all immune markers tested confirmed that 'untreated' birds had significantly reduced expression compared to infected birds. Plating of gut contents from untreated birds confirmed the absence of Campylobacter.

Overall, expression of the immune markers analysed in the infected birds was significantly higher than expression seen in the untreated birds. The pro-inflammatory cytokines IL-6 and IL-8 displayed a large expression in the infected group and this proinflammatory response could help Campylobacter to take advantage of the host immune response and to move and invade outside of the caeca and ileum and move towards organs such as the liver (Humphrey et al., 2014). Other immune markers which could be used to help form a clearer picture in the future of the immune response include IL-22 and IL-23 (Bereswill et al., 2016; Heimesaat et al., 2016). Other factors that may play a role in Campylobacter's ability to proliferate in the gut of chickens may be down to the microbiota, where studies have found a change in microbiota can affect the ability of Campylobacter to grow and spread (Han et al., 2017).

The uninfected birds were observed to produce small quantities of $T_{h} 1, T_{h} 2, T_{h} 17$ and $\mathrm{T}_{\mathrm{h}} 22$ cytokines suggesting that the birds are able to recognise and mount a response to commensal bacteria in the gut which supports work by Crhanova et al (2011) who showed expression of $T_{h} 1, T_{h} 2$ and $T_{h} 17$ in the caecum of noninfected chickens (Crhanova et al., 2011). These levels of expression observed could also be due to other bacteria such as Salmonella and Escherichia coli which have both been shown to induce 
immune responses in chickens (Matulova et al., 2013; Sadeyen et al., 2014). Expression of cytokines such as IL-1 $\beta$, IL-10 and IL-18 has been observed in the caecal tonsils in response to multiple Lactobacilli strains (Brisbin et al., 2010).

The expression of immune markers from the caecal tonsils were then compared to Campylobacter counts from the caeca and ileum of the infected birds. The caeca tonsils lie between the caeca and the ileum and thus Campylobacter counts from these regions could be a good indicator of a correlation of Campylobacter effect in the gut. In both the caeca and the ileum there appeared to be no correlation between the amount of Campylobacter in the gut sections and the expression of biomarkers. The lack of correlation could mean that Campylobacter is only partly responsible for the expression patterns observed as Kers et al (2018) showed that there is a large diversity in chicken microbiota due to host related factors such as age and breed, as well as environment related factors such as housing, litter, feed access, climate and biosecurity and thus the immune responses shown could be in part due to the microbiota and potentially Campylobacters effect on the microbiota (Kers et al., 2018).

\subsection{Conclusion}

This work helps to bridge the gap between results observed in vitro and in vivo, the cocktail of six strains used where able to effectively invade and produce immune responses in human and chicken epithelial cells while also being able to adapt and survive within the infected birds with the host's immune response being up regulated in the infected birds. GWAS analysis was able to find genes uniquelyassociated with Campylobacter strains found in the caeca, ileum and liver with strains from the liver found to be the most invasive in both cell lines. The caecal tonsils were able to provide insight into the important adaptive immune response in chickens. 


\section{Chapter 6 - Discussion}

\subsection{Poultry Industry}

Campylobacter infections have been a cause for concern in the poultry industry for over 40 years despite many interventions and control strategies being suggested to help combat the growing threat (Umaraw et al., 2017). These include preharvest interventions and postharvest interventions such as improved biosecurity on the farm and during processing, including rigorous cleaning regimes. Preharvest interventions are measures of preventing Campylobacter on the farm by reducing the environmental exposure with gumboots, overalls, double foot dips, hand sanitisers and changing room and increasing the poultry's host resistance to reduce Campylobacter in the gut through vaccination (Umaraw et al., 2017).

Postharvest interventions include techniques such as improving hauling and transportation as faeces, feathers and skin are the main sources of contamination during transport, and logistic slaughter where Campylobacter positive flocks after negative flocks to avoid cross contamination (Umaraw et al., 2017).

\subsubsection{Broiler Chickens}

The modern broiler chicken has developed over the past 60 years with the chickens adaptability allowing it to be grown globally under a range of husbandry conditions (Siegel, 2014). Most commercial broilers reach slaughter weight at around four to seven weeks, with some slower growing breeds reaching slaughter weight at a maximum of fourteen weeks (Bessei, 2006). Through selective breeding and feed efficiencies the weight of broiler chickens has steadily increased over time with the average body weight of broilers has increased from 1,400g in 1985 to 2,440g in 2010 (Siegel, 2014). The young age of broiler chickens at slaughter contribute to behaviours and physiology of an immature bird which can lead to many issues ranging from welfare concerns to skeletal dysfunction and malformation (Bessei, 2006). The young age of broiler chickens can also lead to immunosuppression resulting in poor performance of the birds and increased bacterial infections and mortality which can be a large issue for the poultry industry and thus could increase a broiler's susceptibility to Campylobacter infections (Hoerr, 2010; Vaezirad et al., 2016). 


\subsubsection{Influence of Campylobacter on the poultry industry}

No single strain of Campylobacter can represent the entire population of the genus or species and this realisation of 'such diversity' could lead to a change in design of future experiments and trials conducted with industry partnership. In past studies, one strain of Campylobacter has been used to infect chickens in vivo (Smith et al., 2008; Vaezirad et al., 2016), whereas it would be beneficial to infect chickens with a cocktail of strains that better defines the population or a given pathology to gain a better understanding of how Campylobacter pathogenesis in chickens progresses. This may help mimic the natural infection that occurs in commercial systems in an in vivo experiment. Genomic analysis of isolates from naturally infected chickens in chapters 3 and 5 identified important genes that were found to be associated with chicken infection (chapter 3) and the caeca, ileum and liver (chapter 5) through GWAS analysis. Genes identified, such as $\operatorname{murG}, y k k c, h c p A, m a c A$ could make for potential candidates for vaccines as they were found to be associated with strains from the gut. There is currently no vaccine against Campylobacter despite research into potential vaccine targets. Work by Meunier et al (2017) assessed six potential vaccine antigens in Ross broiler chickens which required multiple injections into the birds and reduced Campylobacter loads by 2 to 4.2 $\log _{10}$ CFU/g (Meunier et al., 2017). The requirement of multiple injections into birds would make this unfeasible to commercial chicken companies; however more research into these vaccine candidates could yield promising results in the future and the genes identified in this thesis may provide important targets.

The in vitro analysis of the immune response of $C$. jejuni strains in human and chicken cell lines demonstrated that chicken cells can recognise and mount an immune response to Campylobacter and contributes to the knowledge obtained from previous studies showing that Campylobacter can act as a pathogen in the chicken host (Humphrey et al., 2014; Jennings et al., 2011; Larson et al., 2008). These findings help to challenge the view that Campylobacter are commensals in broiler chickens and will further contribute to changes within the poultry industry leading to new interventions to increase welfare and try to combat Campylobacter levels within flocks.

The use of signalling inhibitors (Chapter 4) demonstrated the importance of pro (ERK) and anti- inflammatory (PI-3 Kinase-AKT) pathways for the invasiveness and $C$. jejuni induced production of IL-8 and IL-10. The larger responses of IL-10 in the chicken cells suggested that the chicken gut may be able to tolerate a larger dose of 
Campylobacter which supports work by Humphrey et al (2014). Induction of a higher anti-inflammatory response in chickens could lead to the animals being able to tolerate larger doses of Campylobacter before showing signs of infection such as diarrhoea and hock marks (Byrne et al., 2007; Williams et al., 2013). Hock marks and pododermatitis are not currently used by the poultry industry as markers for Campylobacter but are used for signs of disease from other bacterial species or poor gut health. The use of ERK signalling inhibitors reduced $C$. jejuni invasion into the chicken cells which supports previous work ( $\mathrm{Hu}$ et al., 2006b; Jin et al., 2003; Konkel et al., 2013; Samuelson and Konkel, 2013). This work could contribute to strategies to reduce Campylobacter numbers in chickens through the use of signalling inhibitors. However, the toxicity of inhibitors to the chickens and their effects on chicken meat would need to be further researched.

Analysis of naturally infected chickens showed that Campylobacter isolated from the liver of infected chickens displayed the highest level of invasion into human and chicken cell lines which supports knowledge that Campylobacter reaching the liver requires a high level of invasion (Jennings et al., 2011; Vaezirad et al., 2016; Williams et al., 2013). The invasiveness of these strains in the liver could lead to more severe human infections from food products such as chicken liver pate and much work has linked Campylobacter infection to this food source (Lahti et al., 2017). However, more work would need to be investigated into rates of infection and Campylobacter found in chicken liver. High levels of Campylobacter in chicken liver may result in the poultry industry having to look at more preventative methods to halt the extra-intestinal spread of Campylobacter.

Experimentally infected chickens displayed elevated levels of the pro-inflammatory cytokines IL-6 and IL-8 in infected versus uninfected birds. Elevation of these cytokines could help Campylobacter to take advantage of the hosts immune response and ultimate leave the gut and move towards organs such as the liver (Humphrey et al., 2014). The poultry industry could look to the betterment of chicken welfare to reduce pro-inflammatory responses and thus could reduce extra-intestinal spread.

\subsection{Campylobacter and public health}

Public Health England have reported a decrease in the number of human cases of Campylobacter infections from 2015 to 2016 by 3,316 cases with the highest number of 
cases affecting people aged 50-59 years old (PHE, 2018). While the decrease in confirmed cases can be seen as a positive, the majority of Campylobacter cases go unreported and the number of confirmed cases is still larger than reported cases in 2007 (PHE, 2018), while in August 2018, confirmed human cases were 5000 higher than over the same period in 2018. Thus Campylobacter is still a major worldwide public health threat with evidence of a global increase in cases (Kaakoush et al., 2015). Analysis of the responses of $C$. jejuni strains in human and chicken epithelial cells, along with data from naturally and experimentally infected chickens showed that human cells may be more sensitive to Campylobacter than chicken ones, which supports previous studies (Humphrey et al., 2014). The ability of chickens to tolerate larger levels is of a concern to public health as a dose as low as $800 \mathrm{CFU}$ of Campylobacter has resulted in diarrhoea, with a dose of 360 CFU causing development of campylobacteriosis in humans (Kaakoush et al., 2015).

There is a need to combat human Campylobacter cases through better understanding of how Campylobacter interacts with chickens and survives in and on chicken tissues. The chicken cells studied were found to employ similar mechanisms in both human and chicken hosts. This finding could lead to new interventions on how to deal with Campylobacter in chickens using inhibitors of pathways in chicken feed which could block Campylobacter invading cells within the chicken and ultimately lead to a reduction of human cases through a reduction of Campylobacter numbers in chickens, however, a limitation would be to make sure the inhibitors are active in the gut of the chickens. Research into the effects of certain inhibitors on chicken health and growth would be essential to make sure there are no adverse effects on the welfare of the birds.

\subsection{Genomic Pipeline}

The genomic analysis throughout this thesis was conducted using the recently developed software Prokka (Seemann, 2014), Roary (Page et al., 2015), Scoary (Brynildsrud et al., 2016) and Gubbins (Croucher et al., 2015). These new pipelines can be used easily and efficiently to analyse large numbers of bacterial strains and produce clear pan-genomes and produce large amounts of data related to the genomes. Prokka and Roary have already been used to analyse Salmonella (Feasey et al., 2017; Petrovska et al., 2016), Legionella (David et al., 2016b) and a number of other bacteria (Chewapreecha et al., 2017; Heinz et al., 2016). Scoary has been used to investigate Helicobacter (van Vliet, 2017) and Bacillus (Bazinet, 2017) with Gubbins also being 
used to measure the recombination in multiple organisms (De Been et al., 2015; Moura et al., 2016; Struve et al., 2015). The widespread use of these new genomic tools contributes to the reliability of the software with results being comparable to older and existing methods.

There are currently many choices available for bioinformatics software such as Ridom, SeqSphere, BIGsdb, Geneious, Piggy, PGAdb and many others. Thus it can be difficult to know which software is the best for any particular work (Jolley and Maiden, 2010; Kearse et al., 2012a; Liu et al., 2016; Mellmann et al., 2011; Thorpe et al., 2018). The major advantage of the recently developed software is that they are open source and therefore free to use; the freedom of availability and ease of their use mean that genomes and bacteria can now be analysed easily, quickly and cheaply, which is beneficial to all areas of bacterial bioinformatics. This has led to more researchers using genomics to understand more about bacterial populations and has expanded the knowledge available to the wider scientific community.

The Roary pipeline developed in this thesis could be used for analysis of the microbiota in chickens and humans however this analysis would take time due to the high number of difference bacteria and sequences which would be extracted from the microbiota. Increasingly there has been a focus on the microbiota and the effect of interactions between this and Campylobacter (Han et al., 2017). Through WGS and the Roary pipeline the microbiota of healthy chickens and ones infected with Campylobacter could be analysed. The genomic differences in expression of certain genes in the bacteria found in the microbiota and from Campylobacter could shed light on interactions between the two. It is unclear whether Campylobacter has an effect on gut microbiota as there are conflicting studies (Connerton et al., 2018; Han et al., 2017). These conflicting results warrant further investigation.

\subsection{Genes Identified}

Genomic analysis in this thesis identified many genes, which could be of interest in the future. The genes pebAl and pldA were found to be present in the core genome of the population of $100 C$. jejuni isolates and that of Campylobacter from naturally infected chickens suggesting that these genes are fundamental for the survival of Campylobacter in different host environments. They are both adhesion-linked genes which play an 
important role in invasion into host cells with deficient mutants invading at a reduced rate (Pei et al., 1998; Ziprin et al., 2001).

GWAS analysis of Campylobacter isolates in this thesis resulted in genes found to be associated with the caeca, ileum and liver of the chicken gut or with specific hosts. Campylobacter isolates from chicken sources were significantly more likely to have the multidrug resistance gene $y k k C$, the regulatory one, $n t r C$ and one for a transferase, murG.. These genes could provide the basis of a diagnostic test to identify if a human Campylobacter case came from chickens or a different source based on gene expression. Using the GWAS results from isolates taken from the caeca, ileum and liver could also help to identify Campylobacter isolates that remain in the gut and ones, which could be more invasive and leave that environment to infect edible tissues. Genes such as acpS or hyaD, which encode for a holo-[acyl-carrier-protein] synthase in C. jejuni and a hyaluronan synthase in $C$. coli, respectively, were found to be associated with strains found in the liver and could be used to identify isolates which have the ability to invade outside the gut and could potentially cause more damage to the chicken host, however there has been little work into the function of these genes and their relationship to invasiveness and virulence in the host.

Comparison of GWAS of potential markers during in vitro pathogenesis and in vivo markers in the caeca, ileum and liver unfortunately did not yield a high overlap of genes with prmA, being the only gene to be found present in both analysis. This is almost certainly due to the Campylobacter strains coming from two very different populations. The gene prmA, which encodes a ribosomal protein methytransferase was found to be associated with intermediate level of expression of IL-8/CXCLi1/2 and the ileum of naturally infected birds. However, there has not been considerable research into the function of this gene in relation to the ability of Campylobacter to invade and infect chickens. While there was only one gene overlap in the GWAS results there where two families of genes which overlapped. The gene $\operatorname{ars} B$ was found to be related to high level of invasion into eptihelial cells, whereas the gene $\operatorname{ars} C$ was found related to strains found in the liver. Both genes relate to a family of genes responsible for arsenic resistance in $C$. jejuni and $C$. coli which have been found to be present in retail meats in very large numbers and so may help survival (Noormohamed and Fakhr, 2013). Another family of genes found in both data sets were the rib family. ribA was found associated with the liver, whereas ribN was associated with low expression of IL-10. 
These genes are related to riboflavin and have been found to be associated with ferric reduction and iron acquisition by $C$. jejuni which could help survival within the blood. Riboflavin has been found to be a virulence factor in Pseudogymnoascus destructans infections as high concentrations were found to be cytotoxic and can enable invasive infections (Crossley et al., 2007; Flieger et al., 2016). These genes may cause a similar pattern in C. jejuni infections. A high number of metabolic genes were found to be associated with each trait analysed and could suggest that survival and ability of Campylobacter to adapt to its surroundings play a large role in virulence.

\subsection{New chicken cell in vitro model}

The MM-ChiC clone 8E11 chicken intestinal cell lines were used to investigate how Campylobacter interacts with chicken hosts with phenotypic outputs of immune markers, toxicity, adhesion and invasion. There are no widespread or widely used in vitro models for chicken systems with most work with Campylobacter using human derived cell lines such as T84 (Zheng et al., 2008), INT407 (Borrmann et al., 2007), HT-29 (Bahrami et al., 2011) and Caco-2 (Man et al., 2010). The lack of a widely used chicken model could lead to large gaps in understanding how Campylobacter behaves in different host environments and so the use of the MM-ChiC clone 8E11 chicken intestinal cell line is a potential candidate for a chicken cell model. The 8E11 cells are derived from leghorn chickens and were confirmed to be chicken 'species' through investigating the expression of chicken MHC in the cells. The 8E11 cells are a robust and easily cultured cell line with the cells having a high growth rate and thus can be used frequently without the need for long incubation times to wait for growth. Campylobacter was shown to be able to invade and induce cytokine expression in the cell line with results being comparable to human cells and previously reported work (Bahrami et al., 2011; Larson et al., 2008; Li et al., 2011). Sequencing of the cells found a small amount of quail DNA within the cells, however work throughout this thesis showing the cells express chicken specific MHCs and chickens TLRs along with work by Shiina et al (2004) who found that quail and chickens have different MHCs led us to confidently call the MM-ChiC clone 8E11 cells chicken intestinal cells (Shiina et al., 2004).

Further work is still needed to fully develop this potential chicken cell model through many different techniques. Analysing the morphology with scanning electron microscopy (SEM) to see if the cells behave in a similar fashion to chicken gut 
epithelial cells along with analysis of cell behaviour with co-culture of leukocytes, which can yield insight in how the epithelial cells are able to interact with the wider immune system. Investigating the TLR profiles of the cells to demonstrate whether they possess chicken specific TLRs-this work has started (Discussion chapter 4). Further understanding of their 'epithelial-like' properties will also be essential (e.g formation of tight junctions). In addition the group is also investigating the potential of $3 \mathrm{D}$ cell culture to grow the cells in an artificially created environment that mimics that of the intestine (Edmondson et al., 2014).

\subsection{Caecal Tonsils}

The caecal tonsils represent the largest lymphoid organs of the chicken GALT and thus play a significant role in inducing and regulating immune responses in the chicken system. The caecal tonsils are a valuable site in the chicken as extraction of RNA from them allows a wealth of data to be analysed through gene expression which has already been investigated with Salmonella (Sadeyen et al., 2004). The caecal tonsils provide information on how the chicken adaptive immune system is activated in response to infection. Many pro- and anti- inflammatory markers were analysed to gain an overview of the immune response which helped support the idea of Campylobacter taking advantage of the host immune response to move and invade from the caeca and ileum, which supports earlier work (Humphrey et al., 2014). A drawback to using caecal tonsils is that they give cytokine levels at a local lymph node but not from the gut epithelium or extra-intestinal organs such as the muscle or more specifically the liver. To gain a better understanding of immune responses to infection caecal tonsils should be analysed in conjunction with 'local biopsy tissues' of chickens and Campylobacter counts in different areas of chicken gut, immune responses in different gut tissues and other organs to gain a more complete view of infection. While this was not possible here the responses assayed in the caecal tonsils warrant future studies where more samples will be collected.

\subsection{Methods}

The major experimental methods used include the bioinformatics analysis of Campylobacter isolates, qPCR of immune responses, invasion assays along with experiments involving growth, biofilm, motility, cell viability and in vivo infection of chickens. Due to lack of available and inexpensive ELISA kits for chickens no comparison could be made between expressions of proteins such as IL-8 which can be 
done easily for human systems. Western blots are a potential experimental method to compare protein expression from both humans and chickens. However, it is difficult to gain precise quantification of protein for comparison with western blots and so qPCR to analyse gene expression was used to provide the most accurate method to compare the human and chicken systems. During the work described in chapter 5 the lab purchased a new PCR machine and worked progressed on the AriaMx Real-time PCR (Agilent, UK) compared to previous work with the BioRad iCycler (Biorad, UK). The analysis of the results differs from those gained during Chapter 4 analysed using the $2^{-\Delta \Delta} \mathrm{Ct}$ method against a housekeeping gene to form a relative expression level (Livak and Schmittgen, 2001). The change in analysis was due to the availability of newer software and hardware. To make the results comparable; samples analysed in Chapter 4 were reanalysed using the AriaMx Real-time PCR and the Pfaffl method. The results yielded comparable results from both methods and are thus reliable. The gentamicin protection assay was thought to be the best method for analysis of invasive potential of Campylobacter isolates due to a lack of alternative methods as well as being a technique widely used to investigate invasion (Friis et al., 2005; Konkel et al., 2013; Pfaffl, 2001; Russell and Blake, 1994).

BIGsdb was originally used to generate MLST data and analysis for the C. jejuni population (Chapter 3). However; due to unforeseen circumstances alternative methods had to be pursued. The Roary pipeline was ultimately chosen for reasons discussed in section 6.3.

\subsection{Limitations}

A limitation during this thesis relates to the 8E11 chicken cells, the cell line was extracted from a leghorn chicken. Chickens used within the poultry industry for meat are broiler chickens and the two breeds behave differently (Williams et al., 2013) and so a cell line from a broiler chicken could potentially give rise to different immune responses and invasion rates. Another limitation during the caecal extraction experimental work (Chapter 5) was with caecal tonsils. They were stored in RNA later to preserve the tissue ready for RNA extraction after conclusion of the study. Some of the caecal tonsils could have been stored in formalin and so could have then been used in histological work to visualise and study the effect of Campylobacter on the tissues themselves. 


\subsection{Further Work}

This project opens up many potential avenues for further research. There have been many genes identified in this thesis though GWAS and gene by gene analysis, their function and effects on the host have to be established. One potential experiment would be to generate mutants of Campylobacter using either C. jejuni NCTC 11168 or M1 and then use these to infect both human and chicken cell lines to compare the effect of the loss of the function knockout in question. During chapter 3, a collection of 100 C. jejuni isolates were used to investigate the population, using the genomic pipeline, analysis of a much larger population of $C$. jejuni and $C$. coli could be undertaken to see if comparable results are shown from a much larger sample size.

Developing the chicken cell model discussed in section 6.5 could also be a very useful experiment which could create a standardised model for in vitro analysis of the chicken system. Development of chicken specific ELISAs to accurately measure production of cytokines in chickens and so compare protein levels post infection in human and chicken systems. This project could be very time consuming and thus may not be feasible depending on resources and time to complete the method. In Chapter 4, signalling and endocytosis pathways were investigated using inhibitors to block downstream signalling molecules. The effect of TLR agonist and antagonists on the chicken cell lines could provide valuable insight into the signalling pathways in chicken systems and whether Campylobacter utilises specific TLRs to invade into cells. The use of TLR agonists and antagonists could also identify potential targets to reduce Campylobacter numbers in chickens through immunomodulatory approaches.

Another avenue to pursue would be to undertake more experiments in vivo, extracting caecal tonsils from infected and uninfected chickens could then be used to understand physiological differences through histology and thus gain a clear idea of the impact Campylobacter has on the tissues of the chicken. Measurement of more immune markers from the caecal tonsils could also be used to help form a clearer picture in the future of the immune responses include IL-22 and -23 (Bereswill et al., 2016; Heimesaat et al., 2016). Mutants created based on the GWAS and phenotypic studies from Chapters 3, 4 and 5 could be used to infect chickens in vivo to analyse the functional diffeences in these genes and whether they are important in Campylobacter invasion to the liver. These studies may uncover markers which could be used for 
diagnostic tests or chicken vaccines to help reduce the threat of Campylobacter in the poultry industry. 


\section{References}

Akiyama, T., Ishida, J., Nakagawa, S., Ogawara, H., Watanabe, S., Itoh, N., et al. (1987). Genistein, a specific inhibitor of tyrosine-specific protein kinases. J. Biol. Chem. 262, 5592-5595.

Al-Banna, N. A., Cyprian, F., and Albert, M. J. (2018). Cytokine responses in campylobacteriosis: Linking pathogenesis to immunity. Cytokine Growth Factor Rev., 1-13. doi:10.1016/j.cytogfr.2018.03.005.

Albert, M. J., Haridas, S., Steer, D., Dhaunsi, G. S., Smith, A. I., and Adler, B. (2007). Identification of a Campylobacter jejuni protein that cross-reacts with cholera toxin. Infect. Immun. 75, 3070-3073. doi:10.1128/IAI.00139-07.

Alessi, D. R., Cuenda, A., Cohen, P., Dudley, D. T., and Saltiel, A. R. (1995). Enzymology : PD 098059 Is a Specific Inhibitor of the Activation of Mitogenactivated Protein Kinase Kinase in Vitro and in Vivo PD 098059 Is a Specific Inhibitor of the Activation of Mitogen-activated Protein Kinase Kinase in Vitro and in Vivo *. 270, 27489-27494. doi:10.1074/jbc.270.46.27489.

Altekruse, S. F., Stern, N. J., Fields, P. I., and Swerdlow, D. L. (1999). Campylobacter jejuni - An emerging foodborne pathogen. Emerg. Infect. Dis. 5, 28-35. doi:10.3201/eid0501.990104.

Ang, C. W., Laman, J. D., Willison, H. J., Wagner, E. R., Endtz, H. P., De Klerk, M. A., et al. (2002). Structure of Campylobacter jejuni lipopolysaccharides determines antiganglioside specificity and clinical features of Guillain-Barr?? and Miller Fisher patients. Infect. Immun. 70, 1202-1208. doi:10.1128/IAI.70.3.12021208.2002.

Archer, N. K., Mazaitis, M. J., Costerton, J. W., Leid, J. G., Powers, M. E., and Shirtliff, M. E. (2011). Properties, regulation and roles in human disease Staphylococcus aureus biofilms. Virulence, 445-459.

Ashgar, S. S. A., Oldfield, N. J., Wooldridge, K. G., Jones, M. A., Irving, G. J., Turner, D. P. J., et al. (2007). CapA, an autotransporter protein of Campylobacter jejuni, mediates association with human epithelial cells and colonization of the chicken gut. J. Bacteriol. 189, 1856-1865. doi:10.1128/JB.01427-06.

Bæk, K. T., Vegge, C. S., and Brøndsted, L. (2011). HtrA chaperone activity contributes to host cell binding in Campylobacter jejuni. Gut Pathog. 3, 13. doi:10.1186/1757-4749-3-13. 
Baggiolini, M. (2015). CXCL8 - The First Chemokine. Front. Immunol. 6, 6-8. doi:10.3389/fimmu.2015.00285.

Bahrami, B., Macfarlane, S., and Macfarlane, G. T. (2011). Induction of cytokine formation by human intestinal bacteria in gut epithelial cell lines. J Appl Microbiol 110, 353-363. doi:10.1111/j.1365-2672.2010.04889.x.

Baldvinsson, S. B., Holst Sørensen, M. C., Vegge, C. S., Clokie, M. R. J., and Brøndsted, L. (2014). Campylobacter jejuni motility is required for infection of the flagellotropic bacteriophage F341. Appl. Environ. Microbiol. 80, 7096-7106. doi:10.1128/AEM.02057-14.

Bamias, G., Arseneau, K., and Cominelli, F. (2014). Cytokines and Mucosal Immunity. Curr. Opin. Gasteroenterology 30, 547-552. doi:10.1097/MOG.0000000000000118.Cytokines.

Banchereau, J., Briere, F., Caux, C., Davoust, J., Lebecque, S., Liu, Y., et al. (2000). Imunobiology of Dendritic Cells. Annu. Rev. Immunol., 767-811.

Bardoň, J., Pudová, V., Koláčková, I., Karpíšková, R., Röderová, M., and Kolář, M. (2017). Virulence and antibiotic resistance genes in campylobacter spp. in the Czech Republic. Epidemiol. Mikrobiol. Imunol. 66, 59-66.

Barjesteh, N., Hodgins, D. C., St. Paul, M., Quinteiro-Filho, W. M., DePass, C., Monteiro, M. A., et al. (2013). Induction of chicken cytokine responses in vivo and in vitro by lipooligosaccharide of Campylobacter jejuni HS:10. Vet. Microbiol. 164, 122-130. doi:10.1016/j.vetmic.2013.02.002.

Barot, M. S., Mosenthal, A. C., and Bokkenheuser, V. D. (1983). Location of Campylobacter-Jejuni in Infected Chicken Livers. J. Clin. Microbiol. 17, 921-922.

Bax, M., Kuijf, M. L., Heikema, A. P., van Rijs, W., Bruijns, S. C. M., García-Vallejo, J. J., et al. (2011). Campylobacter jejuni lipooligosaccharides modulate dendritic cell-mediated $\mathrm{T}$ cell polarization in a sialic acid linkage-dependent manner. Infect. Immun. 79, 2681-2689. doi:10.1128/IAI.00009-11.

Bazinet, A. L. (2017). Pan-genome and phylogeny of Bacillus cereus sensu lato. BMC Evol. Biol. 17, 1-16. doi:10.1186/s12862-017-1020-1.

Beery, J. T., Hugdahl, M. B., and Doyle, M. P. (1988). Colonization of gastrointestinal tracts of chicks by Campylobacter jejuni. Appl. Environ. Microbiol. 54, 23652370 .

Befus, A. D., Johnston, N., Leslie, G. A., and Bienenstock, J. (1980). Gut-Associated Lymphoid Tissue in the Chicken. 125, 2626-2632. 
Bekiaris, V., Persson, E. K., and Agace, W. W. (2014). Intestinal dendritic cells in the regulation of mucosal immunity. Immunol. Rev. 260, 86-101. doi:10.1111/imr.12194.

Bennett Jr., W. E., Gonzalez-Rivera, R., Puente, B. N., Shaikh, N., Stevens, H. J., Mooney, J. C., et al. (2010). Proinflammatory fecal mRNA and childhood bacterial enteric infections. Gut Microbes 1, 209-212. doi:10.4161/gmic.1.4.13004.

Bereswill, S., Alutis, M. E., Grundmann, U., Fischer, A., Göbel, U. B., and Heimesaat, M. M. (2016). Interleukin-18 Mediates Immune Responses to Campylobacter jejuni Infection in Gnotobiotic Mice. PLoS One 11, e0158020. doi:10.1371/journal.pone.0158020.

Berntson, E., Tivemo, M., Engvall, A., Berndtson, E., Tivemo, M., and Engvall, A. (1992). Distribution and Numbers of Campylobacter-in Newly Slaughtered Broiler-Chickens and Hens. Int. J. Food Microbiol. 15, 45-50. doi:10.1016/01681605(92)90134-o.

Bessei, W. (2006). Welfare of broilers: a review. Worlds. Poult. Sci. J. 62, 455-466. doi:10.1079/WPS2005108.

Bishop, A. J. R., and Schiestl, R. H. (2002). Homologous recombination and its role in carcinogenesis. J. Biomed. Biotechnol. 2002, 75-85. doi:10.1155/S1110724302204052.

Boehm, M., Krause-Gruszczynska, M., Rohde, M., Tegtmeyer, N., Takahashi, S., Oyarzabal, O. A., et al. (2011). Major Host Factors Involved in Epithelial Cell Invasion of Campylobacter jejuni: Role of Fibronectin, Integrin Beta1, FAK, Tiam-1, and DOCK180 in Activating Rho GTPase Rac1. Front. Cell. Infect. Microbiol. 1, 17. doi:10.3389/fcimb.2011.00017.

Bolton, D. J. (2015). Campylobacter virulence and survival factors. Food Microbiol. 48, 99-108. doi:10.1016/j.fm.2014.11.017.

Bonneau, L., Gerbeau-Pissot, P., Thomas, D., Der, C., Lherminier, J., Bourque, S., et al. (2010). Plasma membrane sterol complexation, generated by filipin, triggers signaling responses in tobacco cells. Biochim. Biophys. Acta-Biomembr. 1798, 2150-2159. doi:10.1016/j.bbamem.2010.07.026.

Borrmann, E., Berndt, A., Hanel, I., and Kohler, H. (2007). Campylobacter-induced interleukin-8 responses in human intestinal epithelial cells and primary intestinal chick cells. Vet. Microbiol. 124, 115-124. doi:10.1016/j.vetmic.2007.04.041.

Braun, V. (2001). Iron uptake mechanisms and their regulation in pathogenic bacteria. 
Int. J. Med. Microbiol. 291, 67-79. doi:10.1078/1438-4221-00103.

Brisbin, J. T., Gong, J., Parvizi, P., and Sharif, S. (2010). Effects of lactobacilli on cytokine expression by chicken spleen and cecal tonsil cells. Clin. Vaccine Immunol. 17, 1337-1343. doi:10.1128/CVI.00143-10.

Brisbin, J. T., Gong, J., and Sharif, S. (2008). Interactions between commensal bacteria and the gut-associated immune system of the chicken. Anim. Health Res. Rev. 9, 101-110. doi:10.1017/S146625230800145X.

Brøndsted, L., Andersen, M. T., Parker, M., Jørgensen, K., and Ingmer, H. (2005). The HtrA protease of Campylobacter jejuni is required for heat and oxygen tolerance and for optimal interaction with human epithelial cells. Appl. Environ. Microbiol. 71, 3205-3212. doi:10.1128/AEM.71.6.3205-3212.2005.

Bronowski, C., James, C. E., and Winstanley, C. (2014). Role of environmental survival in transmission of Campylobacter jejuni. FEMS Microbiol. Lett. 356, 8-19. doi:10.1111/1574-6968.12488.

Broughton, S. E., Hercus, T. R., Lopez, A. F., and Parker, M. W. (2012). Cytokine receptor activation at the cell surface. Curr. Opin. Struct. Biol. 22, 350-359. doi:10.1016/j.sbi.2012.03.015.

Brown, H. L., Reuter, M., Hanman, K., Betts, R. P., and Van Vliet, A. H. M. (2015). Prevention of biofilm formation and removal of existing biofilms by extracellular dnases of campylobacter jejuni. PLoS One 10, 1-21. doi:10.1371/journal.pone.0121680.

Brynildsrud, O., Bohlin, J., Scheffer, L., and Eldholm, V. (2016). Rapid scoring of genes in microbial pan-genome-wide association studies with Scoary. Genome Biol. 17, 238. doi:10.1186/s13059-016-1108-8.

Buelow, D. R., Christensen, J. E., Neal-Mckinney, J. M., and Konkel, M. E. (2011). Campylobacter jejuni survival within human epithelial cells is enhanced by the secreted protein CiaI. Mol. Microbiol. 80, 1296-1312. doi:10.1111/j.13652958.2011.07645.x.

Butzler, J. P., Dekeyser, P., Detrain, M., and Dehaen, F. (1973). Related vibrio in stool. J. Pediatr. 82, 493-495.

Byrne, C. M., Clyne, M., and Bourke, B. (2007). Campylobacter jejuni adhere to and invade chicken intestinal epithelial cells in vitro. Microbiology 153, 561-569. doi:10.1099/mic.0.2006/000711-0.

Carvalho, A. C. T., Ruiz-palacios, G. M., Ramos-cervantes, P., Cervantes, L., Jiang, X. 
I., and Pickering, L. K. (2001). Molecular Characterization of Invasive and Noninvasive Campylobacter jejuni and Campylobacter coli Isolates. J. Clin. Microbiol. 39, 1353-1359. doi:10.1128/JCM.39.4.1353.

Casteleyn, C., Doom, M., Lambrechts, E., Van den Broeck, W., Simoens, P., and Cornillie, P. (2010). Locations of gut-associated lymphoid tissue in the 3-monthold chicken: a review. Avian Pathol. 39, 143-150.

doi:10.1080/03079451003786105.

Chaguza, C., Cornick, J. E., and Everett, D. B. (2015). Mechanisms and impact of genetic recombination in the evolution of Streptococcus pneumoniae. Comput. Struct. Biotechnol. J. 13, 241-247. doi:10.1016/j.csbj.2015.03.007.

Chang, C., and Miller, J. F. (2006). Campylobacter jejuni colonization of mice with limited enteric flora. Infect. Immun. 74, 5261-5271. doi:10.1128/IAI.01094-05. Chaussade, C., Rewcastle, G. W., Kendall, J. D., Denny, W. A., Cho, K., Gronning, L. M., et al. (2007). Evidence for functional redundancy of class IA PI3K isoforms in insulin signalling. Biochem. J. 404, 449-458. doi:10.1042/BJ20070003.

Chewapreecha, C., Holden, M. T. G., Vehkala, M., Välimäki, N., Yang, Z., Harris, S. R., et al. (2017). Global and regional dissemination and evolution of Burkholderia pseudomallei. Nat Microbiol 2, 1-17. doi:10.1038/nmicrobiol.2016.263.Global.

Christensen, J. E., Pacheco, S. A., and Konkel, M. E. (2009). Identification of a Campylobacter jejuni-secreted protein required for maximal invasion of host cells. Mol. Microbiol. 73, 650-662. doi:10.1111/j.1365-2958.2009.06797.x.

Clevers, H. C., and Bevins, C. L. (2013). Paneth Cells: Maestros of the Small Intestinal Crypts. Annu. Rev. Physiol. 75, 289-311. doi:10.1146/annurev-physiol-030212183744.

Colles, F. M., Dingle, K. E., Cody, A. J., and Maiden, M. C. J. (2008). Comparison of Campylobacter populations in wild geese with those in starlings and free-range poultry on the same farm. Appl. Environ. Microbiol. 74, 3583-3590. doi:10.1128/AEM.02491-07.

Connell, S., Meade, K. G., Allan, B., Lloyd, A. T., Kenny, E., Cormican, P., et al. (2012). Avian resistance to Campylobacter jejuni colonization is associated with an intestinal immunogene expression signature identified by mRNA sequencing. PLoS One 7. doi:10.1371/journal.pone.0040409.

Connerton, P. L., Richards, P. J., Lafontaine, G. M., O’Kane, P. M., Ghaffar, N., Cummings, N. J., et al. (2018). The effect of the timing of exposure to 
Campylobacter jejuni on the gut microbiome and inflammatory responses of broiler chickens. Microbiome 6, 88. doi:10.1186/s40168-018-0477-5.

Cossart, P., and Sansonetti, P. J. (2004). Bacterial Invasion: The Paradigms of Enteroinvasive Pathogens. Science (80-. ). 304, 242-248. doi:10.1126/science.1090124.

Couper, K. N., Blount, D. G., and Riley, E. M. (2008). IL-10: the master regulator of immunity to infection. J. Immunol. 180, 5771-5777. doi:10.4049/jimmunol.180.9.5771.

Crhanova, M., Hradecka, H., Faldynova, M., Matulova, M., Havlickova, H., Sisak, F., et al. (2011). Immune response of chicken gut to natural colonization by gut microflora and to Salmonella enterica serovar enteritidis infection. Infect. Immun. 79, 2755-2763. doi:10.1128/IAI.01375-10.

Crossley, R. A., Gaskin, D. J. H., Holmes, K., Mulholland, F., Wells, J. M., Kelly, D. J., et al. (2007). Riboflavin biosynthesis is associated with assimilatory ferric reduction and iron acquisition by Campylobacter jejuni. Appl. Environ. Microbiol. 73, 7819-7825. doi:10.1128/AEM.01919-07.

Croucher, N. J., Page, A. J., Connor, T. R., Delaney, A. J., Keane, J. A., Bentley, S. D., et al. (2015). Rapid phylogenetic analysis of large samples of recombinant bacterial whole genome sequences using Gubbins. Nucleic Acids Res. 43, e15. doi:10.1093/nar/gku1196.

Dasti, J. I., Tareen, A. M., Lugert, R., Zautner, A. E., and Groß, U. (2010). Campylobacter jejuni: A brief overview on pathogenicity-associated factors and disease-mediating mechanisms. Int. J. Med. Microbiol. 300, 205-211. doi:10.1016/j.ijmm.2009.07.002.

David, J., Dominguez, C., Hamilton, D., and Palena, C. (2016a). The IL-8/IL-8R Axis: A Double Agent in Tumor Immune Resistance. Vaccines 4, 22. doi:10.3390/vaccines4030022.

David, S., Rusniok, C., Mentasti, M., Gomez-valero, L., Harris, S. R., Lechat, P., et al. (2016b). Multiple major disease-associated clones of Legionella pneumophila have emerged recently and independently. Genome Res. 11, 1555-1564.

Davis, L., and DiRita, V. (2008). Growth and Laboratory Maintenance of Campylobacter jejuni. Curr. Protoc. Microbiol. 34, 352-359. doi:10.1177/0963721414541462.Self-Control.

De Been, M., Pinholt, M., Top, J., Bletz, S., Mellmann, A., Van Schaik, W., et al. 
(2015). Core genome multilocus sequence typing scheme for high-resolution typing of enterococcus faecium. J. Clin. Microbiol. 53, 3788-3797. doi:10.1128/JCM.01946-15.

De Boer, P., Wagenaar, J. A., Achterberg, R. P., Van Putten, J. P. M., Schouls, L. M., and Duim, B. (2002). Generation of Campylobacter jejuni genetic diversity in vivo. Mol. Microbiol. 44, 351-359. doi:10.1046/j.1365-2958.2002.02930.x.

De Jong, A. E. I., Van Asselt, E. D., Zwietering, M. H., Nauta, M. J., and De Jonge, R. (2012). Extreme heat resistance of food borne pathogens campylobacter jejuni, escherichia coli, and salmonella typhimurium on chicken breast fillet during cooking. Int. J. Microbiol. 2012. doi:10.1155/2012/196841.

De Zoete, M. R., Keestra, A. M., Roszczenko, P., and Van Putten, J. P. M. (2010). Activation of human and chicken toll-like receptors by Campylobacter spp. Infect. Immun. 78, 1229-1238. doi:10.1128/IAI.00897-09.

Dearlove, B. L., Cody, A. J., Pascoe, B., Méric, G., Wilson, D. J., and Sheppard, S. K. (2016). Rapid host switching in generalist Campylobacter strains erodes the signal for tracing human infections. ISME J. 10, 721-729. doi:10.1038/ismej.2015.149.

DEFRA (2012). Agriculture in the United Kingdom, in Government National Statistics. Dinarello, C. A. (2000). Proinflammatory cytokines. Chest 118, 503-508. doi:10.1378/chest.118.2.503.

Dingle, K. E., Colles, F. M., Falush, D., Maiden, C. J., and Maiden, M. C. J. (2005). Sequence Typing and Comparison of Population Biology of Campylobacter coli and Campylobacter jejuni Sequence Typing and Comparison of Population Biology of Campylobacter coli and Campylobacter jejuni. Society 43, 340-347. doi:10.1128/JCM.43.1.340.

Dingle, K. E., Colles, F. M., Wareing, D. R. A. A., Ure, R., Fox, A. J., Bolton, F. E., et al. (2001). Multilocus Sequence Typing System for Campylobacter jejuni. J. Clin. Microbiol. 39, 14-23. doi:10.1128/JCM.39.1.14.

Doyle, L. (1944). A vibrio associated swine dysentery. Am. J. Vet. Res. 5, 3-5.

Dugar, G., Herbig, A., Forstner, K. U., Heidrich, N., Reinhardt, R., Nieselt, K., et al. (2013). High-resolution transcriptome maps reveal strain-specific regulatory features of multiple Campylobacter jejuni isolates. PLoS Genet 9, e1003495. doi:10.1371/journal.pgen.1003495 PGENETICS-D-13-00603 [pii].

Edmondson, R., Broglie, J. J., Adcock, A. F., and Yang, L. (2014). Three-Dimensional Cell Culture Systems and Their Applications in Drug Discovery and Cell-Based 
Biosensors. Assay Drug Dev. Technol. 12, 207-218. doi:10.1089/adt.2014.573. EFSA (2010). Analysis of the baseline survey on the prevalence of Campylobacter in broiler batches and of Campylobacter and Salmonella on broiler carcasses, in the EU, 2008. ESFA J. 8, 0-132. doi:10.2903/j.efsa.2010.1522.

Eucker, T. P., and Konkel, M. E. (2012). The cooperative action of bacterial fibronectin-binding proteins and secreted proteins promote maximal Campylobacter jejuni invasion of host cells by stimulating membrane ruffling. Cell. Microbiol. 14, 226-238. doi:10.1111/j.1462-5822.2011.01714.x. European Food Standards Agency (2018). Campylobacter. Available at: https://www.food.gov.uk/safety-hygiene/campylobacter?navref=breadcrumb [Accessed May 11, 2018].

European Food Standards Agency, and Agency, E. F. S. (2014). The European Union Summary Report on trends and sources of zoonoses, zoonotic agents and foodborne outbreaks in 2012.

Evans, C. M., and Jenner, R. G. (2013). Transcription factor interplay in t helper cell differentiation. Brief. Funct. Genomics 12, 499-511. doi:10.1093/bfgp/elt025.

Everest, P. H., Goossens, H., Butzler, J. P., Lloyd, D., Knutton, S., Ketley, J. M., et al. (1992). Differentiated Caco-2 cells as a model for enteric invasion by Campylobacter jejuni and C. coli. J Med Microbiol 37, 319-325. doi:10.1099/00222615-37-5-319.

Faber, E., Gripp, E., Maurischat, S., Kaspers, B., Tedin, K., Menz, S., et al. (2015). Novel Immunomodulatory Flagellin-Like Protein FlaC in Campylobacter jejuni and Other Campylobacterales. Host-Microbe Biol. 1, e0002. doi:10.1128/mSphere.00028-15.Editor.

Fagarasan, S., and Honjo, T. (2003). Intestinal IgA synthesis: regulation of front-line body defences. Nat. Rev. Immunol. 3, 63-72. doi:10.1038/nri982.

Fearnhead, P., Yu, S., Biggs, P., Holland, B., and French, N. (2015). Estimating the relative rate of recombination to mutation in bacteria from single-locus variants using composite likelihood methods. Ann. Appl. Stat. 9, 200-224. doi:10.1214/14AOAS795.

Fearnley, C., Manning, G., Bagnall, M., Javed, M. A., Wassenaar, T. M., and Newell, D. G. (2008). Identification of hyperinvasive Campylobacter jejuni strains isolated from poultry and human clinical sources. J. Med. Microbiol. 57, 570-580. doi:10.1099/jmm.0.47803-0. 
Feasey, N. A., Hadfield, J., Keddy, K. H., and Dallman, T. J. (2017). Distinct

Salmonella Enteritidis lineages associated with enterocolitis in high-income settings and invasive disease in low-income settings. Nat Genet 48, 1211-1217. doi:10.1038/ng.3644.Distinct.

Fernando, U., Biswas, D., Allan, B., Willson, P., and Potter, A. A. (2007). Influence of Campylobacter jejuni fliA, rpoN and flgK genes on colonization of the chicken gut. Int. J. Food Microbiol. 118, 194-200. doi:10.1016/j.ijfoodmicro.2007.07.038.

Flanagan, R. C., Neal-McKinney, J. M., Dhillon, A. S., Miller, W. G., and Konkel, M. E. (2009). Examination of Campylobacter jejuni putative adhesins leads to the identification of a new protein, designated FlpA, required for chicken colonization. Infect. Immun. 77, 2399-2407. doi:10.1128/IAI.01266-08.

Flieger, M., Bandouchova, H., Cerny, J., Chudiéková, M., Kolarik, M., Kovacova, V., et al. (2016). Vitamin B2 as a virulence factor in Pseudogymnoascus destructans skin infection. Sci. Rep. 6, 1-12. doi:10.1038/srep33200.

Fonseca, B. B., Santos, I. L., Rossi, D. A., Melo, R. T., Araújo, T. G., Vieira, C. U., et al. (2013). Participation of the Cytoskeletal and Lysosomal Compartments in Campylobacter jejuni Invasion of Caco-2 cells, the Cellular Response by Morphometric Analysis and the Presence of Cytokine and Chemokine Transcripts. Indian J. Microbiol. 53, 155-162. doi:10.1007/s12088-012-0324-5.

Fonseca, B., Santos, J., Coelho, L., Melo, R., Alves, R., and Beletti, M. (2016). Campylobacter Jejuni Increases Transcribed Il-1 and B Causes Morphometric Changes in the Ileal Enterocytes of Chickens. Brazillian J. Poult. Sci. 18, 63-68.

Friis, L. M., Keelan, M., and Taylor, D. E. (2009). Campylobacter jejuni drives MyD88-independent interleukin-6 secretion via toll-like receptor 2. Infect. Immun. 77, 1553-1560. doi:10.1128/IAI.00707-08.

Friis, L. M., Pin, C., Pearson, B. M., and Wells, J. M. (2005). In vitro cell culture methods for investigating Campylobacter invasion mechanisms. J. Microbiol. Methods 61, 145-160. doi:10.1016/j.mimet.2004.12.003.

Garénaux, A., Jugiau, F., Rama, F., De Jonge, R., Denis, M., Federighi, M., et al. (2008). Survival of Campylobacter jejuni strains from different origins under oxidative stress conditions: Effect of temperature. Curr. Microbiol. 56, 293-297. doi:10.1007/s00284-007-9082-8.

Gargi, A., Reno, M., and Blanke, S. R. (2012). Bacterial toxin modulation of the eukaryotic cell cycle: are all cytolethal distending toxins created equally? Front. 
Cell. Infect. Microbiol. 2, 1-11. doi:10.3389/fcimb.2012.00124.

Giepmans, B. N. G., and van IJzendoorn, S. C. D. (2009). Epithelial cell-cell junctions and plasma membrane domains. Biochim. Biophys. Acta - Biomembr. 1788, 820831. doi:10.1016/j.bbamem.2008.07.015.

Göbel, T. W., Kaspers, B., and Stangassinger, M. (2001). NK and T cells constitute two major, functionally distinct intestinal epithelial lymphocyte subsets in the chicken. Int. Immunol. 13, 757-762. doi:10.1093/intimm/13.6.757.

Golden, N. J., and Acheson, D. W. K. (2002). Identification of motility and autoagglutination Campylobacter jejuni mutants by random transposon mutagenesis. Infect. Immun. 70, 1761-1771. doi:10.1128/IAI.70.4.17611771.2002 .

Gomes-Santos, A. C., Moreira, T. G., Castro-Junior, A. B., Horta, B. C., Lemos, L., Cruz, D. N., et al. (2012). New insights into the immunological changes in IL-10deficient mice during the course of spontaneous inflammation in the gut mucosa. Clin. Dev. Immunol. 2012. doi:10.1155/2012/560817.

Gómez Del Moral, M., Fonfría, J., Varas, A., Jiménez, E., Moreno, J., and Zapata, A. G. (1998). Appearance and development of lymphoid cells in the chicken (Gallus gallus) caecal tonsil. Anat. Rec. 250, 182-189. doi:10.1002/(SICI)10970185(199802)250:2<182::AID-AR8>3.0.CO;2-5.

Griekspoor, P., Colles, F. M., McCarthy, N. D., Hansbro, P. M., Ashhurst-Smith, C., Olsen, B., et al. (2013). Marked host specificity and lack of phylogeographic population structure of Campylobacter jejuni in wild birds. Mol. Ecol. 22, 14631472. doi:10.1111/mec.12144.

Guccione, E., Del Rocio Leon-Kempis, M., Pearson, B. M., Hitchin, E., Mulholland, F., Van Diemen, P. M., et al. (2008). Amino acid-dependent growth of Campylobacter jejuni: Key roles for aspartase (AspA) under microaerobic and oxygen-limited conditions and identification of AspB (Cj0762), essential for growth on glutamate. Mol. Microbiol. 69, 77-93. doi:10.1111/j.1365-2958.2008.06263.x.

Guccione, E. J., Kendall, J. J., Hitchcock, A., Garg, N., White, M. A., Mulholland, F., et al. (2017). Transcriptome and proteome dynamics in chemostat culture reveal how Campylobacter jejuni modulates metabolism, stress responses and virulence factors upon changes in oxygen availability. Environ. Microbiol. 19, 4326-4348. doi:10.1111/1462-2920.13930.

Guerry, P. (2007). Campylobacter flagella: not just for motility. Trends Microbiol. 15, 
456-461. doi:10.1016/j.tim.2007.09.006.

Guerry, P., Poly, F., Riddle, M., Maue, A. C., Chen, Y.-H., and Monteiro, M. A. (2012).

Campylobacter Polysaccharide Capsules: Virulence and Vaccines. Front. Cell.

Infect. Microbiol. 2, 1-11. doi:10.3389/fcimb.2012.00007.

Guévremont, E., Nadeau, É., Sirois, M., and Quessy, S. (2006). Antimicrobial susceptibilities of thermophilic Campylobacter from humans, swine, and chicken broilers. Can. J. Vet. Res. 70, 81-86.

Gupta, R. S., Hillier, L. W., Miller, W., Birney, E., Warren, W., Hardison, R. C., et al. (2008). Re-evaluation of chicken CXCR1 determines the true gene structure:

CXCLi1 (K60) and CXCLi2 (CAF/interleukin-8) are ligands for this receptor. $J$. Biol. Chem. 283, 16408-16415. doi:10.1074/jbc.M800998200.

Hadfield, J., Croucher, N. J., Goater, R. J., Abudahab, K., Aanensen, D. M., and Harris, S. R. (2018). Phandango: An interactive viewer for bacterial population genomics. Bioinformatics 34, 292-293. doi:10.1093/bioinformatics/btx610.

Haghighi, H. R., Abdul-Careem, M. F., Dara, R. A., Chambers, J. R., and Sharif, S. (2008). Cytokine gene expression in chicken cecal tonsils following treatment with probiotics and Salmonella infection. Vet. Microbiol. 126, 225-233. doi:10.1016/j.vetmic.2007.06.026.

Hall, B. G., Ehrlich, G. D., and Hu, F. Z. (2010). Pan-genome analysis provides much higher strain typing resolution than multi-locus sequence typing. Microbiology 156, 1060-1068. doi:10.1099/mic.0.035188-0.

Han, Z., Willer, T., Li, L., Pielsticker, C., Rychlik, I., Velge, P., et al. (2017). The influence of the gut microbiota composition on Campylobacter jejuni colonization in chickens. Infect. Immun., IAI.00380-17. doi:10.1128/IAI.00380-17.

Hanel, I., Muller, J., Muller, W., and Schulze, F. (2004). Correlation between invasion of Caco-2 eukaryotic cells and colonization ability in the chick gut in Campylobacter jejuni. Vet Microbiol 101, 75-82. doi:10.1016/j.vetmic.2004.04.004 S0378113504001038 [pii].

Hansson, I., Nyman, A., Lahti, E., Gustafsson, P., and Olsson Engvall, E. (2015). Associations between Campylobacter levels on chicken skin, underlying muscle, caecum and packaged fillets. Food Microbiol. 48, 178-181. doi:10.1016/j.fm.2014.12.013.

Hara, J. R., Feener, T. D., Fischer, C. D., and Buret, A. G. (2012). Campylobacter jejuni disrupts protective toll-like receptor 9 signaling in colonic epithelial cells and 
increases the severity of dextran sulfate sodium-induced colitis in mice. Infect. Immun. 80, 1563-1571. doi:10.1128/IAI.06066-11.

Harada, A., Sekido, N., Akahoshi, T., Wada, T., Mukaida, N., and Matsushima, K. (1994). Essential involvement of interleukin-8 ( IL-8 ) in acute inflammation. 56, $559-564$.

Harris, L. G., Murray, S., Pascoe, B., Bray, J., Meric, G., Magerios, L., et al. (2016). Biofilm morphotypes and population Structure among Staphylococcus epidermidis from commensal and clinical samples. PLoS One 11, 1-15. doi:10.1371/journal.pone.0151240.

Health Protection Agency (2013). UK Standards for Microbiology Investigations Identification of Campylobacter species. Stand. Unit, Microbiol. Serv. ID23, 1-22. doi:ID 7.

Heather, J. M., and Chain, B. (2016). The sequence of sequencers: The history of sequencing DNA. Genomics 107, 1-8. doi:10.1016/j.ygeno.2015.11.003.

Heimesaat, M. M., Alutis, M. E., Grundmann, U., Fischer, A., Göbel, U. B., and Bereswill, S. (2016). The Role of IL-23, IL-22, and IL-18 in Campylobacter Jejuni Infection of Conventional Infant Mice. Eur. J. Microbiol. Immunol. 6, 1-13. doi:10.1556/1886.2016.00008.

Heinz, E., Follador, R., Thomson, N. R., Holt, K. E., Kowarik, M., Wyres, K. L., et al. (2016). The diversity of Klebsiella pneumoniae surface polysaccharides. Microb. Genomics 2. doi:10.1099/mgen.0.000073.

Helleday, T. (2010). Homologous recombination in cancer development, treatment and development of drug resistance. Carcinogenesis 31, 955-960. doi:10.1093/carcin/bgq064.

Hendrixson, D. R., and Dirita, V. J. (2003). Transcription of s54 -dependent but not s28 -dependent flagellar genes in Campylobacter jejuni is associated with formation of the flagellar secretory apparatus. Mol. Microbiol. 50, 687-702. doi:10.1046/j.13652958.2003.3731.x.

Hermans, D., Pasmans, F., Messens, W., Martel, A., Van Immerseel, F., Rasschaert, G., et al. (2012). Poultry as a Host for the Zoonotic Pathogen Campylobacter jejuni. Vector-Borne Zoonotic Dis. 12, 89-98. doi:10.1089/vbz.2011.0676.

Hermans, D., Van Deun, K., Martel, A., Van Immerseel, F., Messens, W., Heyndrickx, M., et al. (2011). Colonization factors of Campylobacter jejuni in the chicken gut. Vet. Res. 42, 82. doi:10.1186/1297-9716-42-82. 
Hickey, T. E., Baqar, S., Bourgeois, A. L., Ewing, C. P., and Guerry, P. (1999).

Campylobacter jejuni-stimulated secretion of interleukin-8 by INT407 cells. Infect. Immun. 67, 88-93.

Hickey, T. E., McVeigh, A. L., Scott, D. A., Michielutti, R. E., Bixby, A., Carroll, S. A., et al. (2000). Campylobacter jejuni cytolethal distending toxin mediates release of interleukin-8 from intestinal epithelial cells. Infect. Immun. 68, 6535-6541. doi:10.1128/IAI.68.12.6535-6541.2000.

Hoerr, F. (2010). Clinical aspects of immunosuppression in poultry. Avian Dis. 54, 2 15. doi:10.2298/VETGL1502091R.

Hofreuter, D., Tsai, J., Watson, R. O., Novik, V., Altman, B., Benitez, M., et al. (2006). Unique features of a highly pathogenic Campylobacter jejuni strain. Infect. Immun. 74, 4694-4707. doi:10.1128/IAI.00210-06.

Hu, L., Bray, M. D., Osorio, M., and Kopecko, D. J. (2006a). Campylobacter jejuni induces maturation and cytokine production in human dendritic cells. Infect Immun 74, 2697-2705. doi:74/5/2697 [pii] 10.1128/IAI.74.5.2697-2705.2006.

Hu, L., McDaniel, J. P., and Kopecko, D. J. (2006b). Signal transduction events involved in human epithelial cell invasion by Campylobacter jejuni 81-176. Microb Pathog 40, 91-100. doi:S0882-4010(05)00138-5 [pii] 10.1016/j.micpath.2005.11.004.

Humphrey, S., Chaloner, G., Kemmett, K., Davidson, N., Williams, N., Kipar, A., et al. (2014). Campylobacter jejuni is not merely a commensal in commercial broiler chickens and affects bird welfare. MBio 5, 1-7. doi:10.1128/mBio.01364-14.

Humphrey, S., Lacharme-Lora, L., Chaloner, G., Gibbs, K., Humphrey, T., Williams, N., et al. (2015). Heterogeneity in the infection biology of Campylobacter jejuni isolates in three infection models reveals an invasive and virulent phenotype in a ST21 isolate from poultry. PLoS One 10, 1-13. doi:10.1371/journal.pone.0141182. Humphrey, T. J., Henley, A., and Lanning, D. G. (1993). The colonization of broiler chickens with Campylobacter jejuni: some epidemiological investigations. Epidemiol. Infect. 110, 601-7. doi:10.1017/S0950268800051025.

Jagannathan, A., Constantinidou, C., and Penn, C. W. (2000). The role of rpoN, ntrC and fliA in flagellar expression in Campylobacter jejuni. Biochem. Soc. Trans. 28, A178. doi:10.1042/bst028a178.

Jain, D., Prasad, K. N., Sinha, S., and Husain, N. (2008). Differences in virulence attributes between cytolethal distending toxin positive and negative Campylobacter 
jejuni strains. J. Med. Microbiol. 57, 267-272. doi:10.1099/jmm.0.47317-0.

Janardhana, V., Broadway, M. M., Bruce, M. P., Lowenthal, J. W., Geier, M. S., Hughes, R. J., et al. (2009). Prebiotics modulate immune responses in the gutassociated lymphoid tissue of chickens. J. Nutr. 139, 1404-1409. doi:10.3945/jn.109.105007.

Jennings, J. L., Sait, L. C., Perrett, C. A., Foster, C., Williams, L. K., Humphrey, T. J., et al. (2011). Campylobacter jejuni is associated with, but not sufficient to cause vibrionic hepatitis in chickens. Vet. Microbiol. 149, 193-199. doi:10.1016/j.vetmic.2010.11.005.

Jin, S., Joe, A., Lynett, J., Hani, E. K., Sherman, P., and Chan, V. L. (2001). JlpA, a novel surface-exposed lipoprotein specific to Campylobacter jejuni, mediates adherence to host epithelial cells. Mol. Microbiol. 39, 1225-1236. doi:10.1046/j.1365-2958.2001.02294.x.

Jin, S., Song, Y. C., Emili, A., Sherman, P. M., and Chan, V. L. (2003). JlpA of Campylobacter jejuni interacts with surface-exposed heat shock protein $90 \alpha$ and triggers signalling pathways leading to the activation of NF- $\kappa \mathrm{B}$ and p38 MAP kinase in epithelial cells. Cell. Microbiol. 5, 165-174. doi:DOI 10.1046/j.14625822.2003.00265.x.

John, D. A., Williams, L. K., Kanamarlapudi, V., Humphrey, T. J., and Wilkinson, T. S. (2017). The Bacterial Species Campylobacter jejuni Induce Diverse Innate Immune Responses in Human and Avian Intestinal Epithelial Cells. Front. Microbiol. 8, 115. doi:10.3389/fmicb.2017.01840.

Jolley, K. A., and Maiden, M. C. J. (2010). BIGSdb: Scalable analysis of bacterial genome variation at the population level. BMC Bioinformatics 11, 1-11. doi:10.1093/nar/gki866.

Jones, M. a, Totemeyer, S., Maskell, D. J., Bryant, C. E., Barrow, P. a, and To, S. (2003). Induction of proinflammatory responses in the human monocytic cell line THP-1 by Campylobacter jejuni. Infect Immun 71, 2626-2633. doi:10.1128/IAI.71.5.2626.

Jorgensen, F., Ellis-Iversen, J., Rushton, S., Bull, S. A., Harris, S. A., Bryan, S. J., et al. (2011). Influence of season and geography on Campylobacter jejuni and C. coli subtypes in housed broiler flocks reared in Great Britain. Appl. Environ. Microbiol. 77, 3741-3748. doi:10.1128/AEM.02444-10.

Joshua, G. W. P., Guthrie-Irons, C., Karlyshev, a V, and Wren, B. W. (2006). Biofilm 
formation in Campylobacter jejuni. Microbiology 152, 387-96. doi:10.1099/mic.0.28358-0.

Joslin, S. N., and Hendrixson, D. R. (2008). Analysis of the Campylobacter jejuni FlgR response regulator suggests integration of diverse mechanisms to activate an NtrClike protein. J. Bacteriol. 190, 2422-2433. doi:10.1128/JB.01827-07.

Joslin, S. N., and Hendrixson, D. R. (2009). Activation of the Campylobacter jejuni FlgSR two-component system is linked to the flagellar export apparatus. $J$. Bacteriol. 191, 2656-2667. doi:10.1128/JB.01689-08.

Kaakoush, N. O., Castano-Rodriguez, N., Mitchell, H. M., Man, S. M., CastañoRodríguez, N., Mitchell, H. M., et al. (2015). Global epidemiology of campylobacter infection. Clin. Microbiol. Rev. 28, 687-720. doi:10.1128/CMR.00006-15.

Kaiser, A., Willer, T., Sid, H., Petersen, H., Baumgärtner, W., Steinberg, P., et al. (2016). Susceptibility of primary chicken intestinal epithelial cells for low pathogenic avian influenza virus and velogenic viscerotropic Newcastle disease virus. Virus Res. 225, 50-63. doi:10.1016/j.virusres.2016.09.001.

Kakuda, T., and DiRita, V. J. (2006). Cj1496c encodes a Campylobacter jejuni glycoprotein that influences invasion of human epithelial cells and colonization of the chick gastrointestinal tract. Infect. Immun. 74, 4715-4723. doi:10.1128/IAI.00033-06.

Kale, A. et al. (2011). The Virulence Factor PEB4 (Cj0596) and the Periplasmic Protein Cj1289 are Two Structurally Related SurA-like Chaperones in the Human Pathogen Camplyobacter jejuni. J. Biol. Chem. 286, 21254-21265.

Kanaya, T., and Ohno, H. (2014). The Mechanisms of M-cell Differentiation. Biosci. Microbiota, Food Heal. 33, 91-97. doi:10.12938/bmfh.2013-024.

Kannaki, T. R., Reddy, M. R., Shanmugam, M., Verma, P. C., and Sharma, R. P. (2010). Chicken toll-like receptors and their role in immunity. Worlds. Poult. Sci. J. 66, 727-738. doi:10.1017/S0043933910000693.

Karst, A. M., Dai, D. L., Cheng, J. Q., and Li, G. (2006). Role of p53 up-regulated modulator of apoptosis and phosphorylated Akt in melanoma cell growth, apoptosis, and patient survival. Cancer Res. 66, 9221-9226. doi:10.1158/00085472.CAN-05-3633.

Kawai, T., and Akira, S. (2009). The roles of TLRs, RLRs and NLRs in pathogen recognition. Int. Immunol. 21, 317-337. doi:10.1093/intimm/dxp017. 
Kawasaki, T., and Kawai, T. (2014). Toll-like receptor signaling pathways. Front. Immunol. 5, 1-8. doi:10.3389/fimmu.2014.00461.

Kearse, M., Moir, R., Wilson, A., Stones-Havas, S., Cheung, M., Sturrock, S., et al. (2012a). Geneious Basic: An integrated and extendable desktop software platform for the organization and analysis of sequence data. Bioinformatics 28, 1647-1649. doi:10.1093/bioinformatics/bts199.

Kearse, M., Moir, R., Wilson, A., Stones-Havas, S., Cheung, M., Sturrock, S., et al. (2012b). Geneious version 10.1.3. http://www.geneious.com.

Keestra, A. M., de Zoete, M. R., Bouwman, L. I., Vaezirad, M. M., and Van Putten, J. P. M. (2013). Unique features of chicken Toll-like receptors. Dev. Comp. Immunol. 41, 316-323. doi:10.1016/j.dci.2013.04.009.

Kers, J. G., Velkers, F. C., Fischer, E. A. J., Hermes, G. D. A., Stegeman, J. A., and Smidt, H. (2018). Host and environmental factors affecting the intestinal microbiota in chickens. Front. Microbiol. 9, 1-14. doi:10.3389/fmicb.2018.00235.

Khan, I. et al (2005). PorA protein of Campylobacter jejuni is not a cytotoxin mediating inflammatory diarrhoea. Microbes Infect., 853-859.

Killiny, N., and Almeida, R. P. P. (2011). Gene regulation mediates host specificity of a bacterial pathogen. Environ. Microbiol. Rep. 3, 791-797. doi:10.1111/j.17582229.2011.00288.x.

Kim, Y. S., and Ho, S. B. (2010). Intestinal goblet cells and mucins in health and disease: Recent insights and progress. Curr. Gastroenterol. Rep. 12, 319-330. doi:10.1007/s11894-010-0131-2.

Kitagawa, H., Hiratsuka, Y., Imagawa, T., and Uehara, M. (1998). Distribution of lymphoid tissue in the caecal mucosa of chickens. J. Anat. 192 ( Pt 2, 293-298. doi:10.1046/j.1469-7580.1998.19220293.x.

Kittl, S., Heckel, G., Korczak, B. M., and Kuhnert, P. (2013). Source attribution of human Campylobacter isolates by MLST and Fla-typing and association of genotypes with quinolone resistance. PLoS One 8, 1-8. doi:10.1371/journal.pone.0081796.

Klunder, L. J., Faber, K. N., Dijkstra, G., and Van Ijzendoorn, S. C. D. (2017). Mechanisms of cell polarity - Controlled epithelial homeostasis and immunity in the intestine. Cold Spring Harb. Perspect. Biol. 9, 1-16. doi:10.1101/cshperspect.a027888.

Kogut, M. H., He, H., and Kaiser, P. (2005). Lipopolysaccharide binding 
protein/CD14/TLR4-dependent recognition of Salmonella LPS induces the functional activation of chicken heterophils and up-regulation of pro-inflammatory cytokine and chemokine gene expression in these cells. Anim. Biotechnol. 16, 165181. doi:10.1080/10495390500264896.

Konkel, M. E., and Cieplak Jr., W. (1992). Altered synthetic response of Campylobacter jejuni to cocultivation with human epithelial cells is associated with enhanced internalization. Infect Immun 60, 4945-4949. Available at: http://www.ncbi.nlm.nih.gov/pubmed/1399005.

Konkel, M. E., Garvis, S. G., Tipton, S. L., Anderson Jr., D. E., and Cieplak Jr., W. (1997). Identification and molecular cloning of a gene encoding a fibronectinbinding protein (CadF) from Campylobacter jejuni. Mol. Microbiol. 24, 953-963. doi:10.1046/j.1365-2958.1997.4031771.x.

Konkel, M. E., Kim, B. J., Rivera-Amill, V., and Garvis, S. G. (1999). Bacterial secreted proteins are required for the internaliztion of Campylobacter jejuni into cultured mammalian cells. Mol. Microbiol. 32, 691-701. doi:10.1046/j.13652958.1999.01376.x.

Konkel, M. E., Klena, J. D., Rivera-amill, V., Monteville, M. R., Biswas, D., Raphael, B., et al. (2004). Secretion of Virulence Proteins from Campylobacter jejuni is Dependent on a Functional Flagellar Export Apparatus. J. Bacteriol. 186, 32963303. doi:10.1128/JB.186.11.3296.

Konkel, M. E., Samuelson, D. R., Eucker, T. P., Shelden, E. a, and O’Loughlin, J. L. (2013). Invasion of epithelial cells by Campylobacter jejuni is independent of caveolae. Cell Commun. Signal. 11, 100. doi:10.1186/1478-811X-11-100.

Koolman, L., Whyte, P., Burgess, C., and Bolton, D. (2016). Virulence gene expression, adhesion and invasion of Campylobacter jejuni exposed to oxidative stress (H2O2). Int. J. Food Microbiol. 220, 33-38. doi:10.1016/j.ijfoodmicro.2016.01.002.

Kovanen, S. M., Kivistö, R. I., Rossi, M., Schott, T., Kärkkäinen, U. M., Tuuminen, T., et al. (2014). Multilocus sequence typing (MLST) and whole-genome MLST of campylobacter jejuni isolates from human infections in three districts during a seasonal peak in Finland. J. Clin. Microbiol. 52, 4147-4154. doi:10.1128/JCM.01959-14.

Krause-Gruszczynska, M., Rohde, M., Hartig, R., Genth, H., Schmidt, G., Keo, T., et al. (2007). Role of the small Rho GTPases Rac1 and Cdc42 in host cell invasion of 
Campylobacter jejuni. Cell. Microbiol. 9, 2431-2444. doi:10.1111/j.14625822.2007.00971.x.

Kuhn, R., Lohler, J., Rennick, D., Rajewsky, K., and Muller, W. (1993). Interleukin-10deficient mice develop chronic enterocolitis. Cell 75, 263-274. doi:10.1016/00928674(93)80068-P.

Kunisawa, J., and Kiyono, H. (2013). Immune regulation and monitoring at the epithelial surface of the intestine. Drug Discov. Today 18, 87-92. doi:10.1016/j.drudis.2012.08.001.

Lahti, E., Lofdahl, M., Agren, J., Hansson, I., Olsson Engvall, E., Löfdahl, M., et al. (2017). Confirmation of a Campylobacteriosis Outbreak Associated with Chicken Liver Pate Using PFGE and WGS. Zoonoses Public Health 64, 14-20. doi:10.1111/zph.12272.

Lai, C.-H. C.-K., Chen, Y.-A., Lin, C.-J., Lin, H.-J. H., Kao, M.-C., Huang, M.-Z., et al. (2016). Molecular Mechanisms and Potential Clinical Applications of Campylobacter jejuni Cytolethal Distending Toxin. Front. Cell. Infect. Microbiol. 6, 1-8. doi:10.3389/fcimb.2016.00009.

Larson, C. L., Shah, D. H., Dhillon, A. S., Call, D. R., Ahn, S., Haldorson, G. J., et al. (2008). Campylobacter jejuni invade chicken LMH cells inefficiently and stimulate differential expression of the chicken CXCLi1 and CXCLi2 cytokines. Microbiology 154, 3835-3847. doi:10.1099/mic.0.2008/021279-0.

Lavelle, E. C., Murphy, C., O’Neill, L. A. J., and Creagh, E. M. (2010). The role of TLRs, NLRs, and RLRs in mucosal innate immunity and homeostasis. Mucosal Immunol. 3, 17-28. doi:10.1038/mi.2009.124.

Lee, M. S., Johansen, L., Zhang, Y., Wilson, A., Keegan, M., Avery, W., et al. (2007). The novel combination of chlorpromazine and pentamidine exerts synergistic antiproliferative effects through dual mitotic action. Cancer Res. 67, 11359-11367. doi:10.1158/0008-5472.CAN-07-2235.

Lee, R. B., Hassane, D. C., Cottle, D. L., Pickett, L., and Pickett, C. L. (2003). Interactions of Campylobacter jejuni Cytolethal Distending Toxin Subunits CdtA and $\mathrm{CdtC}$ with HeLa Cells Interactions of Campylobacter jejuni Cytolethal Distending Toxin Subunits CdtA and CdtC with HeLa Cells. Infect. Immun. 71, 4883-4890. doi:10.1128/IAI.71.9.4883.

Lefebure, T., Bitar, P. D. P., Suzuki, H., and Stanhope, M. J. (2010). Evolutionary dynamics of complete Campylobacter pan-genomes and the bacterial species 
concept. Genome Biol. Evol. 2, 646-655. doi:10.1093/gbe/evq048.

Lertsethtakarn, P., Ottemann, K. M., and Hendrixson, D. R. (2011). Motility and chemotaxis in Campylobacter and Helicobacter. Annu. Rev. Microbiol. 65, 389410. doi:10.1146/annurev-micro-090110-102908.

Letunic, I., and Bork, P. (2016). Interactive tree of life (iTOL) v3: an online tool for the display and annotation of phylogenetic and other trees. Nucleic Acids Res. 44, W242-W245. doi:10.1093/nar/gkw290.

Levy, S. E., and Myers, R. M. (2016). Advancements in Next-Generation Sequencing. Annu. Rev. Genomics Hum. Genet. 17, 95-115. doi:10.1146/annurev-genom083115-022413.

Li, Y.-P., Vegge, C. S., Brøndsted, L., Madsen, M., Ingmer, H., and Bang, D. D. (2011). Campylobacter jejuni induces an anti-inflammatory response in human intestinal epithelial cells through activation of phosphatidylinositol 3-kinase/Akt pathway. Vet. Microbiol. 148, 75-83. doi:10.1016/j.vetmic.2010.08.009.

Li, Y. P., Ingmer, H., Madsen, M., and Bang, D. D. (2008). Cytokine responses in primary chicken embryo intestinal cells infected with Campylobacter jejuni strains of human and chicken origin and the expression of bacterial virulence-associated genes. BMC Microbiol. 8, 1-10. doi:10.1186/1471-2180-8-107.

Lim, B. J., Lee, D., Hong, S. W., and Jeong, H. J. (2011). Toll-like receptor 4 signaling is involved in IgA-stimulated mesangial cell activation. Yonsei Med. J. 52, 610615. doi:10.3349/ymj.2011.52.4.610.

Lin, C. D., Lai, C. K., Lin, Y. H., Hsieh, J. T., Sing, Y. T., Chang, Y. C., et al. (2011). Cholesterol depletion reduces entry of Campylobacter jejuni cytolethal distending toxin and attenuates intoxication of host cells. Infect Immun 79, 3563-3575. doi:10.1128/IAI.05175-11 IAI.05175-11 [pii].

Liongue, C., Sertori, R., and Ward, A. C. (2016). Evolution of Cytokine Receptor Signaling. J. Immunol. 197, 11-18. doi:10.4049/jimmunol.1600372.

Liu, Y. Y., Chiou, C. S., and Chen, C. C. (2016). PGAdb-builder: A web service tool for creating pan-genome allele database for molecular fine typing. Sci. Rep. 6, 1-5. doi:10.1038/srep36213.

Livak, K. J., and Schmittgen, T. D. (2001). Analysis of relative gene expression data using real-time quantitative PCR and. Methods 25, 402-408. doi:10.1006/meth.2001.1262.

Luber, P., and Bartelt, E. (2007). Enumeration of Campylobacter spp. on the surface 
and within chicken breast fillets. J. Appl. Microbiol. 102, 313-318.

doi:10.1111/j.1365-2672.2006.03105.x.

Luckheeram, R. V., Zhou, R., Verma, A. D., and Xia, B. (2012). CD4+T Cells:

Differentiation and Functions. Clin. Dev. Immunol. 2012, 1-12.

doi:10.1155/2012/925135.

MacCallum, A. J., Harris, D., Haddock, G., and Everest, P. H. (2006). Campylobacter jejuni-infected human epithelial cell lines vary in their ability to secrete interleukin-8 compared to in vitro-infected primary human intestinal tissue. Microbiology 152, 3661-3665. doi:10.1099/mic.0.29234-0.

Macia, E., Ehrlich, M., Massol, R., Boucrot, E., Brunner, C., and Kirchhausen, T. (2006). Dynasore, a Cell-Permeable Inhibitor of Dynamin. Dev. Cell 10, 839-850. doi:10.1016/j.devcel.2006.04.002.

Mack, D., Siemssen, N., and Laufs, R. (1992). Parallel induction by glucose of adherence and a polysaccharide antigen specific for plastic-adherent Staphylococcus epidermidis: Evidence for functional relation to intercellular adhesion. Infect. Immun. 60, 2048-2057.

Magajna, B. A., and Schraft, H. (2015). Campylobacter jejuni biofilm cells become viable but non-culturable (VBNC) in low nutrient conditions at $4{ }^{\circ} \mathrm{C}$ more quickly than their planktonic counterparts. Food Control 50, 45-50. doi:10.1016/j.foodcont.2014.08.022.

Maiden, M. C. J., Rensburg, M. J. J., Bray, J. E., Earle, S. G., Ford, S. A., Jolley, K. A., et al. (2013). MLST revisited: the gene-by-gene approach to bacterial genomics. Nat Rev Microbiol. 2013 11, 728-736. doi:10.1038/nrmicro3093.MLST.

Man, S. M., Kaakoush, N. O., Leach, S. T., Nahidi, L., Lu, H. K., Norman, J., et al. (2010). Host attachment, invasion, and stimulation of proinflammatory cytokines by Campylobacter concisus and other non-Campylobacter jejuni Campylobacter species. J Infect Dis 202, 1855-1865. doi:10.1086/657316.

Marotta, F., Garofolo, G., Di Donato, G., Aprea, G., Platone, I., Cianciavicchia, S., et al. (2015). Population Diversity of Campylobacter jejuni in Poultry and Its Dynamic of Contamination in Chicken Meat. Biomed Res. Int. 2015. doi:10.1155/2015/859845.

Matsunami, H., Barker, C. S., Yoon, Y. H., Wolf, M., and Samatey, F. A. (2016). Complete structure of the bacterial flagellar hook reveals extensive set of stabilizing interactions. Nat. Commun. 7, 1-10. doi:10.1038/ncomms13425. 
Matulova, M., Varmuzova, K., Sisak, F., Havlickova, H., Babak, V., Stejskal, K., et al. (2013). Chicken innate immune response to oral infection with Salmonella enterica serovar Enteritidis. Vet. Res. 44, 1. doi:10.1186/1297-9716-44-37.

May, J. A., Ratan, H., Glenn, J. R., Lösche, W., Spangenberg, P., and Heptinstall, S. (1998). GPIIb-IIIa antagonists cause rapid disaggregation of platelets pre-treated with cytochalasin D. Evidence that the stability of platelet aggregates depends on normal cytoskeletal assembly. Platelets 9, 227-32. doi:10.1080/09537109876744.

McCarthy, N. D., Colles, F. M., Dingle, K. E., Bagnall, M. C., Manning, G., Maiden, M. C. J., et al. (2007). Host-associated genetic import in Campylobacter jejuni. Emerg. Infect. Dis. 13, 267-272. doi:10.3201/eid1302.060620.

Mcdermott, A. J., and Huffnagle, G. B. (2014). The microbiome and regulation of mucosal immunity. Immunology 142, 24-31. doi:10.1111/imm.12231.

Medini, D., Donati, C., Tettelin, H., Masignani, V., and Rappuoli, R. (2005). The microbial pan-genome. Curr. Opin. Genet. Dev. 15, 589-594. doi:10.1016/j.gde.2005.09.006.

Meinersmann, R. J., and Hiett, K. L. (2000). Concerted evolution of duplicate fla genes in Campylobacter. Microbiology 146, 2283-2290.

Mellmann, A., Harmsen, D., Cummings, C. A., Zentz, E. B., Leopold, S. R., Rico, A., et al. (2011). Prospective genomic characterization of the german enterohemorrhagic Escherichia coli O104:H4 outbreak by rapid next generation sequencing technology. PLoS One 6. doi:10.1371/journal.pone.0022751.

Méric, G., Miragaia, M., De Been, M., Yahara, K., Pascoe, B., Mageiros, L., et al. (2015). Ecological overlap and horizontal gene transfer in Staphylococcus aureus and Staphylococcus epidermidis. Genome Biol. Evol. 7, 1313-1328. doi:10.1093/gbe/evv066.

Méric, G., Yahara, K., Mageiros, L., Pascoe, B., Maiden, M. C. J., Jolley, K. A., et al. (2014). A reference pan-genome approach to comparative bacterial genomics: Identification of novel epidemiological markers in pathogenic Campylobacter. PLoS One 9. doi:10.1371/journal.pone.0092798.

Merino, S., and Tomás, J. M. (2014). Gram-negative flagella glycosylation. Int. J. Mol. Sci. 15, 2840-2857. doi:10.3390/ijms15022840.

Meunier, M., Guyard-Nicodème, M., Vigouroux, E., Poezevara, T., Beven, V., Quesne, S., et al. (2017). Promising new vaccine candidates against Campylobacter in broilers. PLoS One 12, 1-14. doi:10.1371/journal.pone.0188472. 
Miller, W. G., Chapman, M. H., Yee, E., On, S. L. W., McNulty, D. K., Lastovica, A. J., et al. (2012). Multilocus Sequence Typing Methods for the Emerging Campylobacter Species C. hyointestinalis, C. lanienae, C. sputorum, C. concisus, and C. curvus. Front. Cell. Infect. Microbiol. 2, 1-12. doi:10.3389/fcimb.2012.00045.

Mills, D. C., Gundogdu, O., Elmi, A., Bajaj-Elliott, M., Taylor, P. W., Wren, B. W., et al. (2012). Increase in Campylobacter jejuni invasion of intestinal epithelial cells under low-oxygen coculture conditions that reflect the in vivo environment. Infect. Immun. 80, 1690-1698. doi:10.1128/IAI.06176-11.

Min, T., Vedadi, M., Watson, D. C., Wasney, G. A., Munger, C., Cygler, M., et al. (2009). Specificity of campylobacter jejuni adhesin PEB3 for phosphates and structural differences among its ligand complexes. Biochemistry 48, 3057-3067. doi:10.1021/bi802195d.

Mohammed, K. A. S., Miles, R. J., and Halablab, M. A. (2004). The pattern and kinetics of substrate metabolism of Campylobacter jejuni and Campylobacter coli. Lett. Appl. Microbiol. 39, 261-266. doi:10.1111/j.1472-765X.2004.01574.x.

Morley, L., McNally, A., Paszkiewicz, K., Corander, J., Méric, G., Sheppard, S. K., et al. (2015). Gene Loss and Lineage-Specific Restriction-Modification Systems Associated with Niche Differentiation in the Campylobacter jejuni Sequence Type 403 Clonal Complex. Appl. Environ. Microbiol. 81, 3641-3647. doi:10.1128/AEM.00546-15.

Moura, A., Criscuolo, A., Pouseele, H., Maury, M., Leclercq, A., Tarr, C., et al. (2016). Whole genome-based population biology and epidemiological surveillance of Listeria monocytogenes To cite this version : Nat. Microbiol. 2, 1-10.

Muir, W. I., Bryden, W. L., and Husband, A. J. (2000). Immunity, vaccination and the avian intestinal tract. Dev. Comp. Immunol. 24, 325-342. doi:10.1016/S0145305X(99)00081-6.

Murphy, C., Carroll, C., and Jordan, K. N. (2006). Environmental survival mechanisms of the foodborne pathogen Campylobacter jejuni. J. Appl. Microbiol. 100, 623632. doi:10.1111/j.1365-2672.2006.02903.x.

Murphy, K., Travers, P., and Walport, M. (2007). Janeway's Immunobiology. 7th ed. Garland Science.

Naikare, H., Palyada, K., Panciera, R., Marlow, D., and Stintzi, A. (2006). Major role for FeoB in Campylobacter jejuni ferrous iron acquisition, gut colonization, and 
intracellular survival. Infect. Immun. 74, 5433-5444. doi:10.1128/IAI.00052-06.

Nakajima, T., Kuribayashi, T., Yamamoto, S., Moore, J. E., Millar, B. C., and Matsuda, M. (2014). Molecular analysis of superoxide dismutase in Campylobacter lari. Ann. Microbiol. 64, 1347-1356. doi:10.1007/s13213-013-0778-7.

Namin, B. M., Dallal, M. M. S., and Daryani, N. E. (2015). The effect of Campylobacter concisus on expression of IL-18, TNF- $\alpha$ and p53 in barrett's cell lines. Jundishapur J. Microbiol. 8. doi:10.5812/jjm.26393.

Neal-McKinney, J. M., and Konkel, M. E. (2012). The Campylobacter jejuni CiaC virulence protein is secreted from the flagellum and delivered to the cytosol of host cells. Front. Cell. Infect. Microbiol. 2, 31. doi:10.3389/fcimb.2012.00031.

Negretti, N. M., Gourley, C. R., Clair, G., Adkins, J. N., and Konkel, M. E. (2017). The food-borne pathogen Campylobacter jejuni responds to the bile salt deoxycholate with countermeasures to reactive oxygen species. Sci. Rep. 7, 1-11. doi:10.1038/s41598-017-15379-5.

Neill, S. D., Campbell, J. N., and Greene, J. A. (1984). Campylobacter species in broiler chickens. Avian Pathol 13, 777-785. doi:784672709 [pii] 10.1080/03079458408418574.

Noormohamed, A., and Fakhr, M. K. (2013). Arsenic resistance and prevalence of arsenic resistance genes in Campylobacter jejuni and Campylobacter coli isolated from retail meats. Int. J. Environ. Res. Public Health 10, 3453-3464. doi:10.3390/ijerph10083453.

O Cróinín, T., and Backert, S. (2012). Host epithelial cell invasion by Campylobacter jejuni: trigger or zipper mechanism? Front. Cell. Infect. Microbiol. 2, 25. doi:10.3389/fcimb.2012.00025.

Obeng, A. S., Rickard, H., Sexton, M., Pang, Y., Peng, H., and Barton, M. (2012). Antimicrobial susceptibilities and resistance genes in Campylobacter strains isolated from poultry and pigs in Australia. J. Appl. Microbiol. 113, 294-307. doi:10.1111/j.1365-2672.2012.05354.x.

Oeckinghaus, A., and Ghosh, S. (2009). The NF- k B Family of Transcription Factors and Its Regulation. Cold Spring Harb. Perspect. Biol. 1, 1-14.

Ogden, I. D., Dallas, J. F., MacRae, M., Rotariu, O., Reay, K. W., Leitch, M., et al. (2009). Campylobacter excreted into the environment by animal sources: prevalence, concentration shed, and host association. Foodborne Pathog. Dis. 6, 1161-1170. doi:10.1089/fpd.2009.0327. 
Oláh, I., Glick, B., and Taylor, R. L. (1984). Meckel's diverticulum. II. A novel lymphoepithelial organ in the chicken. Anat. Rec. 208, 253-263. doi:10.1002/ar.1092080212.

Oláh, I., Nagy, N., and Vervelde, L. (2013). Structure of the Avian Lymphoid System. doi:10.1016/B978-0-12-396965-1.00002-9.

Olmos, G., and Lladó, J. (2014). Tumor necrosis factor alpha: A link between neuroinflammation and excitotoxicity. Mediators Inflamm. 2014. doi:10.1155/2014/861231.

On, S. (2001). Taxonomy of Campylobacter, Arcobacter, Helicobacter and related bacteria: current status, future prospects and immediate concerns. Soc. Appl. Microbiol. 30, 1-15.

Opal, S. M., and DePalo, V. A. (2000). Anti-inflammatory cytokines. Chest 117, 1162 1172. doi:10.1378/chest.117.4.1162.

Owen, J., Punt, J., Stranford, S., and Jones, P. (2013). Kuby Immunology. 7th ed. W. H. Freeman.

Page, A. J., Cummins, C. A., Hunt, M., Wong, V. K., Reuter, S., Holden, M. T. G., et al. (2015). Roary: Rapid large-scale prokaryote pan genome analysis.

Bioinformatics 31, 3691-3693. doi:10.1093/bioinformatics/btv421.

Palomo, J., Dietrich, D., Martin, P., Palmer, G., and Gabay, C. (2015). The interleukin (IL)-1 cytokine family - Balance between agonists and antagonists in inflammatory diseases. Cytokine 76, 25-37. doi:10.1016/j.cyto.2015.06.017.

Palyada, K., Threadgill, D., and Stintzi, A. (2004). Iron Acquisition and Regulation in Campylobacter jejuni Iron Acquisition and Regulation in Campylobacter jejuni. $J$. Bacteriol. 186, 4714-4729. doi:10.1128/JB.186.14.4714.

Pan, D., and Yu, Z. (2014). Intestinal microbiome of poultry and its interaction with host and diet. Gut Microbes 5, 108-119. doi:10.4161/gmic.26945.

Parkhill, J., Wren, B. W., Mungall, K., Ketley, J. M., Churcher, C., Basham, D., et al. (2000). The genome sequence of the food-borne pathogen Campylobacter jejuni reveals hypervariable sequences. Nature 403, 665-668. doi:10.1038/35001088.

Parte, A. C. (2014). LPSN - List of prokaryotic names with standing in nomenclature. Nucleic Acids Res. 42, 613-616. doi:10.1093/nar/gkt1111.

Pascoe, B., Méric, G., Murray, S., Yahara, K., Mageiros, L., Bowen, R., et al. (2015). Enhanced biofilm formation and multi-host transmission evolve from divergent genetic backgrounds in Campylobacter jejuni. Environ. Microbiol. 17, 4779-4789. 
doi:10.1111/1462-2920.13051.

Pei, Z., and Blaser, M. J. (1993). PEB1, the major cell-binding factor of Campylobacter jejuni, is a homolog of the binding component in gram-negative nutrient transport systems. J. Biol. Chem. 268, 18717-18725.

Pei, Z., Burucoa, C., Grignon, B., Baqar, S., Huang, X. Z., Kopecko, D. J., et al. (1998). Mutation in the peb1A locus of Campylobacter jejuni reduces interactions with epithelial cells and intestinal colonization of mice. Infect. Immun. 66, 938-943.

Perez-Lopez, A., Behnsen, J., Nuccio, S. P., and Raffatellu, M. (2016). Mucosal immunity to pathogenic intestinal bacteria. Nat. Rev. Immunol. 16, 135-148. doi:10.1038/nri.2015.17.

Petrovska, L., Mather, A. E., Abuoun, M., Branchu, P., Harris, S. R., Connor, T., et al. (2016). Microevolution of monophasic Salmonella typhimurium during epidemic, United Kingdom, 2005-2010. Emerg. Infect. Dis. 22, 617-624. doi:10.3201/eid2204.150531.

Pfaffl, M. W. (2001). A new mathematical model for relative quantification in. Nucleic Acid Res. 29, 16-21. doi:10.1093/nar/29.9.e45.

PHE (2016). Campylobacter data 2006 to 2015.

PHE (2017). Zoonoses Overview Report. Available at: https://www.gov.uk/government/uploads/system/uploads/attachment_data/file/664 448/UK_Zoonoses_report_2016.pdf.

PHE (2018). Salmonella data 2007 to 2016. Public Heal. Engl.

Pickett, C. L., Pesci, E. C., Cottle, D. L., Russell, G., and Erdem, A. N. (1996). Prevalence of cytolethal distending toxin production in Campylobacter jejuni and relatedness of Campylobacter sp . cdtB gene. These include : Prevalence of Cytolethal Distending Toxin Production in Campylobacter jejuni and Relatedness of Campylobacter sp. Infect. Immun. 64, 2070-2078.

Pielsticker, C., Glünder, G., and Rautenschlein, S. (2012). Colonization properties of Campylobacter jejuni in chickens. Eur. J. Microbiol. Immunol. (Bp). 2, 61-5. doi:10.1556/EuJMI.2.2012.1.9.

Poly, F. and P. G. (2008). Pathogenesis of Campylobacter. Curr. Opin. Gasteroenterology 24, 27-31.

Poly, F., Ewing, C., Goon, S., Hickey, T. E., Rockabrand, D., Majam, G., et al. (2007). Heterogeneity of a Campylobacter jejuni protein that is secreted through the flagellar filament. Infect. Immun. 75, 3859-3867. doi:10.1128/IAI.00159-07. 
Poly, F., Serichatalergs, O., Schulman, M., Ju, J., Cates, C. N., Kanipes, M., et al. (2011). Discrimination of major capsular types of Campylobacter jejuni by multiplex PCR. J. Clin. Microbiol. 49, 1750-1757. doi:10.1128/JCM.02348-10.

Posada, D., Crandall, K. A., and Holmes, E. C. (2002). Recombination in Evolutionary Genomics. Annu. Rev. Genet. 36, 75-97.

doi:10.1146/annurev.genet.36.040202.111115.

Punyadarsaniya, D., Winter, C., Mork, A. K., Amiri, M., Naim, H. Y., Rautenschlein, S., et al. (2015). Precision-cut intestinal slices as a culture system to analyze the infection of differentiated intestinal epithelial cells by avian influenza viruses. $J$ Virol Methods 212, 71-75. doi:10.1016/j.jviromet.2014.10.015 S01660934(14)00416-9 [pii].

Purdy, D., Cawthraw, S., Dickinson, J. H., Newell, D. G., and Park, S. F. (1999). Generation of a superoxide dismutase (SOD)-deficient mutant of Campylobacter coli: Evidence for the significance of SOD in Campylobacter survival and colonization. Appl. Environ. Microbiol. 65, 2540-2546.

Raghuwanshi, S., Su, Y., Singh, V., Hayes, K., Richmond, A., and Richardson, R. (2012). The chemokine receptors CXCR1 and CXCR2 couple to distinct G protein-coupled receptor kinases to mediate and regulate leukocyte functions Sandeep. J. Immunol. 189, 1-25. doi:10.1007/s10439-011-0452-9.Engineering.

Rams, T. E., Feik, D., and Slots, J. (1993). Campylobacter rectus in human periodontitis. Oral Microbiol. Immunol. 8, 230-235. doi:10.1111/j.1399302X.1993.tb00565.x.

Rathbun, K. M., and Thompson, S. A. (2009). Mutation of PEB4 alters the outer membrane protein profile of Campylobacter jejuni. FEMS Microbiol. Lett. 300, 188-194. doi:10.1111/j.1574-6968.2009.01795.x.

Rathinam, V. A. K., Appledorn, D. M., Hoag, K. A., Amalfitano, A., and Mansfield, L. S. (2009). Campylobacter jejuni-induced activation of dendritic cells involves cooperative signaling through toll-like receptor 4 (TLR4)-MyD88 and TLR4-TRIF axes. Infect. Immun. 77, 2499-2507. doi:10.1128/IAI.01562-08.

Reeser, R. J., Medler, R. T., Billington, S. J., Jost, B. H., and Joens, L. A. (2007). Characterization of Campylobacter jejuni biofilms under defined growth conditions. Appl. Environ. Microbiol. 73, 1908-1913. doi:10.1128/AEM.00740-06. Reid, W. D. K., Close, A. J., Humphrey, S., Chaloner, G., Lacharme-Lora, L., Rothwell, L., et al. (2016). Cytokine responses in birds challenged with the human food- 
borne pathogen Campylobacter jejuni implies a Th17 response. $R$. Soc. Open Sci. 3, 150541. doi:10.1098/rsos.150541.

Ribot, E. M., Fitzgerald, C., Kubota, K., Swaminathan, B., and Barrett, T. J. (2001). Rapid Pulsed-Field Gel Electrophoresis protocol for subtyping of Campylobacter jejuni. J. Clin. Microbiol. 39, 1889-1894. doi:10.1128/JCM.39.5.1889.

Rivera-Amill, V., Kim, B. J., Seshu, J., and Konkel, M. E. (2001). Secretion of the Virulence-Associated Campylobacter Invasion Antigens from Campylobacter jejuni Requires a Stimulatory Signal. J. Infect. Dis. 183, 1607-1616. doi:10.1086/320704.

Rohde, F., Schusser, B., Hron, T., Farkasova, H., Plachy, J., Hartle, S., et al. (2018).

Characterization of Chicken Tumor Necrosis Factor- $\alpha$, a Long Missed Cytokine in Birds. Front. Immunol. 9, 1-14. doi:10.3389/fimmu.2018.00605.

Roop, R. M., Smibert, R. M., Johnson, J. L., and Krieg, N. R. (1985). Campylobacter mucosalis (Lawson, Leaver, Pettigrew, and Rowland 1981) comb. nov.: Emended Description. Int. J. Syst. Bacteriol. 35, 189-192. doi:10.1099/00207713-35-2-189. Rosenquist, H., Boysen, L., Galliano, C., Nordentoft, S., Ethelberg, S., and Borck, B. (2009). Danish strategies to control Campylobacter in broilers and broiler meat: facts and effects. Epidemiol. Infect. 137, 1742-1750.

doi:10.1017/S0950268809002684.

Rosner, B. M., Schielke, A., Didelot, X., Kops, F., Breidenbach, J., Willrich, N., et al. (2017). A combined case-control and molecular source attribution study of human Campylobacter infections in Germany, 2011-2014. Sci. Rep., 1-12. doi:10.1038/s41598-017-05227-x.

Ruiz-Palacios, G. M. (2007). The Health Burden of Campylobacter Infection and the Impact of Antimicrobial Resistance: Playing Chicken. Clin. Infect. Dis. 44, 701703. doi:10.1086/509936.

Ruiz-palacios, G. M., Escamilla, E., and Torres, N. (1981). Experimental Campylobacter Diarrhea in Chickens. Infect. Immun. 34, 250-255.

Russell, R. G., and Blake, D. C. (1994). Cell association and invasion of Caco-2 cells by Campylobacter jejuni. Infect. Immun. 62, 3773-3779.

Sadeyen, J., Kaiser, P., Stevens, M., and Dziva, F. (2014). Analysis of immune responses induced by avian pathogenic Escherichia coli infection in turkeys and their association with resistance to homologous re-challenge. Vet. Res. 45, 1-12. doi:10.1186/1297-9716-45-19. 
Sadeyen, J. R., Trotereau, J., Velge, P., Marly, J., Beaumont, C., Barrow, P. A., et al. (2004). Salmonella carrier state in chicken: Comparison of expression of immune response genes between susceptible and resistant animals. Microbes Infect. 6, 1278-1286. doi:10.1016/j.micinf.2004.07.005.

Samuelson, D. R., Eucker, T. P., Bell, J. a, Dybas, L., Mansfield, L. S., and Konkel, M. E. (2013). The Campylobacter jejuni CiaD effector protein activates MAP kinase signaling pathways and is required for the development of disease. Cell Commun. Signal. 11, 79. doi:10.1186/1478-811X-11-79.

Samuelson, D. R., and Konkel, M. E. (2013). Serine phosphorylation of cortactin is required for maximal host cell invasion by Campylobacter jejuni. Cell Commun Signal 11, 82. doi:10.1186/1478-811X-11-82 1478-811X-11-82 [pii].

Sandilands, V., Whyte, F., Williams, L. K., Wilkinson, T. S., Sparks, N. H. C., and Humphrey, T. J. (2018). Reliably colonising broiler chickens with Campylobacter spp. using a litter-based method. Br. Poult. Sci. 0, 00071668.2018.1523538. doi:10.1080/00071668.2018.1523538.

Sanyal, S. C., Islam, K. M. N., and Neogy, P. K. B. (1984). Campylobacter jejuni diarrhea model in infant chickens. Infect. Immun. 43, 931-936.

Scherer, K., Bartelt, E., Sommerfield, C., Hildebrandt, G., Sommerfeld, C., and Hildebrandt, G. (2006). Quantification of Campylobacter on the surface and in the muscle of chicken legs at retail. J. Food Prot. 69, 757-761. doi:10.4315/0362028X-69.4.757.

Schoenborn, J. R., and Wilson, C. B. (2007). Regulation of Interferon- $\gamma$ During Innate and Adaptive Immune Responses. Adv. Immunol. 96, 41-101. doi:10.1016/S00652776(07)96002-2.

Schwarze, K., Buchanan, J., Taylor, J. C., and Wordsworth, S. (2018). Are wholeexome and whole-genome sequencing approaches cost-effective? A systematic review of the literature. Genet. Med. 00. doi:10.1038/gim.2017.247.

Seemann, T. (2014). Prokka: Rapid prokaryotic genome annotation. Bioinformatics 30, 2068-2069. doi:10.1093/bioinformatics/btu153.

Shahzad, A., Knapp, M., Lang, I., and Köhler, G. (2010). Interleukin 8 (IL-8) - a universal biomarker? Int. Arch. Med. 3, 11. doi:10.1186/1755-7682-3-11.

Shebl, F. M., Pinto, L. A., García-Piñeres, A., Lempicki, R., Williams, M., Harro, C., et al. (2010). Comparison of mRNA and protein measures of cytokines following vaccination with human papillomavirus-16 L1 virus-like particles. Cancer 
Epidemiol. Biomarkers Prev. doi:10.1158/1055-9965.EPI-10-0064.

Sheppard, S. K., Colles, F. M., McCarthy, N. D., Strachan, N. J. C., Ogden, I. D.,

Forbes, K. J., et al. (2011). Niche segregation and genetic structure of

Campylobacter jejuni populations from wild and agricultural host species. Mol.

Ecol. 20, 3484-3490. doi:10.1111/j.1365-294X.2011.05179.x.

Sheppard, S. K., Colles, F., Richardson, J., Cody, A. J., Elson, R., Lawson, A., et al.

(2010a). Host association of campylobacter genotypes transcends geographic

variations. Appl. Environ. Microbiol. 76, 5269-5277. doi:10.1128/AEM.00124-10.

Sheppard, S. K., Dallas, J. F., Wilson, D. J., Strachan, N. J. C., McCarthy, N. D., Jolley,

K. A., et al. (2010b). Evolution of an agriculture-associated disease causing

Campylobacter coli clade: Evidence from national surveillance data in Scotland.

PLoS One 5, 1-9. doi:10.1371/journal.pone.0015708.

Sheppard, S. K., Didelot, X., Meric, G., Torralbo, A., Jolley, K. a, Kelly, D. J., et al.

(2013). Genome-wide association study identi fi es vitamin B 5 biosynthesis as a

host speci fi city factor in Campylobacter. Proc. Natl. Acad. Sci. U. S. A. 110, 1-5.

doi:10.5061/dryad.28n35.

Sheppard, S. K., Jolley, K. A., and Maiden, M. C. J. (2012). A gene-by-gene approach

to bacterial population genomics: Whole genome MLST of Campylobacter. Genes

(Basel). 3, 261-277. doi:10.3390/genes3020261.

Sheppard, S. K., McCarthy, N. D., Falush, D., and Maiden, M. C. J. (2008).

Convergence of Campylobacter species: implications for bacterial evolution.

Science (80-. ). 320, 237-239. doi:10.1126/science.1155532.

Shiina, T., Shimizu, S., Hosomichi, K., Kohara, S., Watanabe, S., Hanzawa, K., et al. (2004). Comparative Genomic Analysis of Two Avian (Quail and Chicken) MHC Regions. J. Immunol. 172, 6751-6763. doi:10.4049/jimmunol.172.11.6751.

Sick, C., Schneider, K., Staeheli, P., and Weining, K. C. (2000). Novel chicken CXC and CC chemokines. Cytokine 12, 181-186. doi:10.1006/cyto.1999.0543 S10434666(99)90543-4 [pii].

Siegel, P. B. (2014). Evolution of the Modern Broiler and Feed Efficiency. Annu. Rev.

Anim. Biosci. 2, 375-385. doi:10.1146/annurev-animal-022513-114132.

Silva, J., Leite, D., Fernandes, M., Mena, C., Gibbs, P. A., and Teixeira, P. (2011).

Campylobacter spp. As a foodborne pathogen: A review. Front. Microbiol. 2, 112. doi:10.3389/fmicb.2011.00200.

Skarp, C. P. A., Hanninen, M. L., and Rautelin, H. I. K. (2016). Campylobacteriosis: 
The role of poultry meat. Clin. Microbiol. Infect. 22, 103-109. doi:10.1016/j.cmi.2015.11.019.

Skirrow, M. B. (1977). Campylobacter enteritis: A “new” disease. Br. Med. J. 2, 9-11. doi:10.1136/bmj.2.6078.9.

Skirrow, M. B. (2006). John McFadyean and the centenary of the first isolation of Campylobacter species. Clin. Infect. Dis. 43, 1213-1217. doi:10.1086/508201.

Smith, C. K., Abuoun, M., Cawthraw, S. A., Humphrey, T. J., Rothwell, L., Kaiser, P., et al. (2008). Campylobacter colonization of the chicken induces a proinflammatory response in mucosal tissues. FEMS Immunol. Med. Microbiol. 54, 114-121. doi:10.1111/j.1574-695X.2008.00458.x.

Smith, C. K., Kaiser, P., Rothwell, L., Humphrey, T., Barrow, P. a, and Jones, M. a (2005). Campylobacter jejuni -Induced Cytokine Responses in Avian Cells. Infect. Immun. 73, 2094-2100. doi:10.1128/IAI.73.4.2094.

Smith, J. M., Smith, N. H., O’Rourke, M., and Spratt, B. G. (1993). How clonal are bacteria? Proc. Natl. Acad. Sci. U. S. A. 90, 4384-4388. doi:10.1073/pnas.90.10.4384.

Stella, V. J., and He, Q. (2008). Cyclodextrins. Toxicol. Pathol. 36, 30-42. doi:10.1177/0192623307310945.

Stintzi, A., Marlow, D., Palyada, K., Panciera, R., Whitworth, L., Clarke, C., et al. (2005). Use of Genome-Wide Expression Profiling and Mutagenesis To Study the Intestinal Lifestyle of Campylobacter jejuni Use of Genome-Wide Expression Profiling and Mutagenesis To Study the Intestinal Lifestyle of Campylobacter jejuni. Society 73, 1797-1810. doi:10.1128/IAI.73.3.1797.

Struve, C., Roe, C. C., Stegger, M., Stahlhut, S. G., Hansen, D. S., Engelthaler, D. M., et al. (2015). Mapping the evolution of hypervirulent Klebsiella pneumoniae. MBio 6, 1-12. doi:10.1128/mBio.00630-15.

Sturlan, S., Oberhuber, G., Beinhauer, B. G., Tichy, B., Kappel, S., Wang, J., et al. (2001). Interleukin-10-deficient mice and inflammatory bowel disease associated cancer development. Carcinogenesis 22, 665-671. doi:10.1093/carcin/22.4.665.

Su, D. L., Lu, Z. M., Shen, M. N., Li, X., and Sun, L. Y. (2012). Roles of pro- and antiinflammatory cytokines in the pathogenesis of SLE. J. Biomed. Biotechnol. 2012. doi:10.1155/2012/347141.

Szymanski, C. M., Burr, D. H., and Guerry, P. (2002). Campylobacter Protein Glycosylation Affects Host Cell Interactions. Infect. Immun. 70, 2242-2244. 
doi:10.1128/IAI.70.4.2242.

Taha-abdelaziz, K., Alkie, T. N., Hodgins, D. C., Shojadoost, B., and Sharif, S. (2016). Characterization of host responses induced by Toll-like receptor ligands in chicken cecal tonsil cells. Vet. Immunol. Immunopathol. 174, 19-25. doi:10.1016/j.vetimm.2016.04.002.

Takeda, K., and Akira, S. (2005). Toll-like receptors in innate immunity. Int. Immunol. 17, 1-14. doi:10.1093/intimm/dxh186.

Tamura, A., Kitano, Y., Hata, M., Katsuno, T., Moriwaki, K., Sasaki, H., et al. (2008). Megaintestine in Claudin-15-Deficient Mice. Gastroenterology 134, 523-534. doi:10.1053/j.gastro.2007.11.040.

Tamura, K., Stecher, G., Peterson, D., Filipski, A., and Kumar, S. (2013). MEGA6: Molecular evolutionary genetics analysis version 6.0. Mol. Biol. Evol. 30, 27252729. doi:10.1093/molbev/mst197.

Tanaka, T., Narazaki, M., and Kishimoto, T. (2014). IL-6 in inflammation, immunity, and disease. Cold Spring Harb. Perspect. Biol. 6, a016295. doi:10.1101/cshperspect.a016295.

Teh, A. H. T., Lee, S. M., and Dykes, G. A. (2014). Does campylobacter jejuni form biofilms in food-related environments? Appl. Environ. Microbiol. 80, 5154-5160. doi:10.1128/AEM.01493-14.

Thelestam, M., and Frisan, T. (2004). Cytolethal distending toxins. Rev Physiol Biochem Pharmacol 152, 111-133. doi:10.1007/s10254-004-0030-8.

Thorpe, H. A., Bayliss, S. C., Sheppard, S. K., and Feil, E. J. (2018). Piggy: a rapid, large-scale pan-genome analysis tool for intergenic regions in bacteria. Gigascience 7, 1-11. doi:10.1093/gigascience/giy015.

Tolker-Neilsen, T. (2015). Biofilm Development. Microbiol. Spectr. 3, 1-12. doi:10.1128/microbiolspec.MB-0001-2014.

Ulluwishewa, D., Anderson, R. C., McNabb, W. C., Moughan, P. J., Wells, J. M., and Roy, N. C. (2011). Regulation of Tight Junction Permeability by Intestinal Bacteria and Dietary Components. J. Nutr. 141, 769-776. doi:10.3945/jn.110.135657.

Umaraw, P., Prajapati, A., Verma, A. K., Pathak, V., and Singh, V. P. (2017). Control of campylobacter in poultry industry from farm to poultry processing unit: A review. Crit. Rev. Food Sci. Nutr. 57, 659-665. doi:10.1080/10408398.2014.935847.

Vaezirad, M. M., Keestra-Gounder, A. M., de Zoete, M. R., Koene, M. G., Wagenaar, J. 
A., and van Putten, J. P. M. (2016). Invasive behavior of Campylobacter jejuni in immunosuppressed chicken. Virulence 0, 1-13.

doi:10.1080/21505594.2016.1221559.

van der Flier, L. G., and Clevers, H. (2009). Stem Cells, Self-Renewal, and

Differentiation in the Intestinal Epithelium. Annu. Rev. Physiol. 71, 241-260. doi:10.1146/annurev.physiol.010908.163145.

van der Walt, M., Spencer, B., and Loveday, R. (1988). The first isolations of Campylobacter mucosalis from pigs in South Africa. Onderstepoort J. Vet. Res. $55,165-8$.

van Tonder, A. J., Mistry, S., Bray, J. E., Hill, D. M. C., Cody, A. J., Farmer, C. L., et al. (2014). Defining the Estimated Core Genome of Bacterial Populations Using a Bayesian Decision Model. PLoS Comput. Biol. 10, e1003788. doi:10.1371/journal.pcbi.1003788.

van Vliet, A. H. M. (2017). Use of pan-genome analysis for the identification of lineage-specific genes of Helicobacter pylori. FEMS Microbiol. Lett. 364, 1-7. doi:10.1093/femsle/fnw296.

Vandamme, P., Debruyne, L., De Brandt, E., and Falsen, E. (2010). Reclassification of Bacteroides ureolyticus as Campylobacter ureolyticus comb. nov., and emended description of the genus Campylobacter. Int. J. Syst. Evol. Microbiol. 60, 20162022. doi:10.1099/ijs.0.017152-0.

Vaughn, L. E., Holt, P. S., Moore, R. W., and Gast, R. K. (2006). Enhanced Gross Visualization of Chicken Peyer's Patch: Novel Staining Technique Applied to Fresh Tissue Specimens. Avian Dis. 50, 298-302. doi:10.1637/7467-110305R.1.

Velayudhan, J., Jones, M. a, Barrow, P. a, and Kelly, D. J. (2004). L -Serine Catabolism via an Oxygen-Labile L -Serine Dehydratase Is Essential for Colonization of the Avian Gut by Campylobacter jejuni L -Serine Catabolism via an Oxygen-Labile L -Serine Dehydratase Is Essential for Colonization of the Avian Gut by Campyl. Infect. Immun. 72, 260-268. doi:10.1128/IAI.72.1.260.

Vernikos, G., Medini, D., Riley, D. R., and Tettelin, H. (2015). Ten years of pangenome analyses. Curr. Opin. Microbiol. 23, 148-154. doi:10.1016/j.mib.2014.11.016.

Vos, M., and Didelot, X. (2009). A comparison of homologous recombination rates in bacteria and archaea. ISME J. 3, 199-208. doi:10.1038/ismej.2008.93.

Walter, M. R. (2014). The Molecular Basis of IL-10 Function: From Receptor Structure 
to the Onset of Signalling. 380, 1-21. doi:10.1007/978-3-662-43492-5.

Wang, L., Li, J., Jr, J. L., Li, R. X., Lv, C. F., Li, S., et al. (2016). Identification of the Paneth cells in chicken small intestine. 1-5.

Wang, Y., and Taylor, D. E. (1990). Natural transformation in Campylobacter species. J. Bacteriol. 172, 949-955.

Wassenaar, T. M. (1997). Toxin production by Campylobacter spp. Clin. Microbiol. Rev. 10, 466-476.

Watson, R. O., and Galan, J. E. (2005). Signal transduction in Campylobacter jejuniinduced cytokine production. Cell Microbiol 7, 655-665. doi:CMI498 [pii] 10.1111/j.1462-5822.2004.00498.x.

Watson, R. O., and Galán, J. E. (2008). Campylobacter jejuni survives within epithelial cells by avoiding delivery to lysosomes. PLoS Pathog. 4, 0069-0083. doi:10.1371/journal.ppat.0040014.

Weingarten, R. A., Grimes, J. L., and Olson, J. W. (2008). Role of Campylobacter jejuni respiratory oxidases and reductases in host colonization. Appl. Environ. Microbiol. 74, 1367-1375. doi:10.1128/AEM.02261-07.

Westfall, H. N., Rollins, D. M., and Weiss, E. (1986). Substrate utilization by Campylobacter jejuni and Campylobacter coli. Appl.Environ.Microbiol. 52, 700705. Available at: pm:2877615.

WHO (2012). The global view of Campylobacteriosis. doi:ISBN 9789241564601.

WHO (2018). Campylobacter. Available at: http://www.who.int/en/news-room/factsheets/detail/campylobacter [Accessed May 11, 2018].

Whyte, R., Hudson, J. A., and Graham, C. (2006). Campylobacter in chicken livers and their destruction by pan frying. Lett. Appl. Microbiol. 43, 591-595. doi:10.1111/j.1472-765X.2006.02020.x.

Williams, L. K., Sait, L. C., Trantham, E. K., Cogan, T. a, and Humphrey, T. J. (2013). Campylobacter infection has different outcomes in fast- and slow-growing broiler chickens. Avian Dis. 57, 238-41. doi:10.1637/10442-110212-Reg.1.

Williams, L. K., Trantham, E. K., and Cogan, T. A. (2014). "Conditional commensalism of Campylobacter in poultry," in In Sheppard SK $(E d)$ Campylobacter Ecology and Evolution. Caister Academic Press.

Wilson, D. J., Gabriel, E., Leatherbarrow, A. J. H., Cheesbrough, J., Gee, S., Bolton, E., et al. (2009). Rapid evolution and the importance of recombination to the gastroenteric pathogen Campylobacter jejuni. Mol. Biol. Evol. 26, 385-397. 
doi:10.1093/molbev/msn264.

Wong, A., Lange, D., Houle, S., Arbatsky, N. P., Valvano, M. A., Knirel, Y. A., et al. (2015). Role of capsular modified heptose in the virulence of Campylobacter jejuni. Mol. Microbiol. 96, 1136-1158. doi:10.1111/mmi.12995.

Yasuda, M., Tanaka, S., Arakawa, H., Taura, Y., Yokomizo, Y., and Ekino, S. (2002). A comparative study of gut-associated lymphoid tissue in calf and chicken. Anat. Rec. 266, 207-217. doi:10.1002/ar.10062.

Yoshimura, T. (2015). Discovery of IL-8/CXCL8 (The Story from Frederick). Front. Immunol. 6, 6-8. doi:10.3389/fimmu.2015.00278.

Yu, J., Blom, J., Glaeser, S. P., Jaenicke, S., Juhre, T., Rupp, O., et al. (2017). A review of bioinformatics platforms for comparative genomics. Recent developments of the EDGAR 2.0 platform and its utility for taxonomic and phylogenetic studies. $J$. Biotechnol. 261, 2-9. doi:10.1016/j.jbiotec.2017.07.010.

Yurong, Y., Ruiping, S., Shimin, Z., and Yibao, J. (2005). Effect of probiotics on intestinal mucosal immunity and ultrastructure of cecal tonsils of chickens. Arch. Anim. Nutr. 59, 237-246. doi:10.1080/17450390500216928.

Zdanov, A. (2010). Structural analysis of cytokines comprising the IL-10 family. Cytokine Growth Factor Rev. 21, 325-330. doi:10.1016/j.cytogfr.2010.08.003.

Zhang, J.-M., and An, J. (2009). Cytokines, Inflammation and Pain. Int. Anesthesiol. Clin. 69, 482-489. doi:10.1097/AIA.0b013e318034194e.Cytokines.

Zheng, J., Meng, J., Zhao, S., Singh, R., and Song, W. (2008). Campylobacter-induced interleukin-8 secretion in polarized human intestinal epithelial cells requires Campylobacter-secreted cytolethal distending toxin- and toll-like receptormediated activation of NF-??B. Infect. Immun. 76, 4498-4508. doi:10.1128/IAI.01317-07.

Zhu, J., Yamane, H., and Paul, W. (2010). Differentiation of effector CD4 T cell populations. Anпu Rev Immunol. 28, 445-489. doi:10.1146/annurev-immunol030409-101212.Differentiation.

Zilbauer, M., Dorrell, N., Wren, B. W., and Bajaj-Elliott, M. (2008). Campylobacter jejuni-mediated disease pathogenesis: an update. Trans. R. Soc. Trop. Med. Hyg. 102, 123-129. doi:10.1016/j.trstmh.2007.09.019.

Ziprin, R. L., Young, C. R., Byrd, J. a, Stanker, L. H., Hume, M. E., Gray, S. a, et al. (2001). Role of Campylobacter jejuni potential virulence genes in cecal colonization. Avian Dis. 45, 549-557. doi:10.2307/1592894. 
Zucca, E., and Bertoni, F. (2016). The spectrum of MALT lymphoma at different sites: biological and therapeutic relevance. Blood 127, 2082-2092. doi:10.1182/blood2015-12-624304.

\section{Appendix and Supplementary Data}

\subsection{Optimisation of qPCR}

Studies in the HT-29 human intestinal cells informed our work in 8E11 chicken intestinal cells with regard to time points used. In addition, there is a relative paucity of ELISAs/antibodies available for chicken cytokines and thus transcriptional responses were used to compare human and chicken cells.

To compare the transcriptional inflammatory responses of human and chicken gut epithelial cells, the 100 Campylobacter strains (Table 2.2) were used to infect HT-29, human intestinal epithelial cells and 8E11 chicken intestinal epithelial cells. Time point experiments suggested that 24 hours was the optimal for IL- 8 responses and thus RNA was extracted from HT-29 and 8E11 at this time point. Three separate experiments were completed $(n=3)$ and the RNA from the M1 infected samples used to optimise primers conditions.

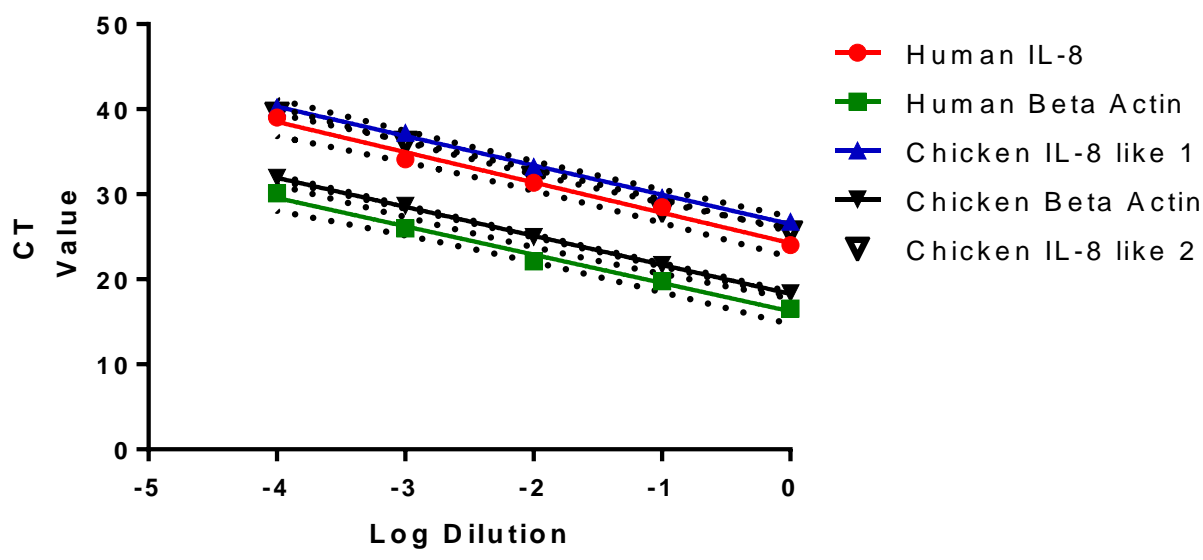

Figure 8.1 standard curves showing the efficiency of Real time primers used in this study for Human IL-8 and $\beta$-actin and Chicken IL-8 and $\beta$-actin. 
Transcriptional analysis of the inflammatory response was determined by measuring RNA message for human IL-8, chicken IL-8-like 1 , human $\beta$-Actin and chicken $\beta$ Actin. Primer efficiency was established by amplification of a dilution series of reverse transcribed total RNA isolated from human and chicken cells infected with the M1 reference strain of Campylobacter (Figure 8.1). Primer efficiencies were $90.8 \%$ for the Human IL-8 primers; $99.49 \%$ for the Human $\beta$-Actin primers; $94.77 \%$ for the Chicken IL-8 Like 1 primers, $97.45 \%$ for the Chicken IL-8 like 2 primers and $96.37 \%$ efficiency for the Chicken $\beta$-actin primers.

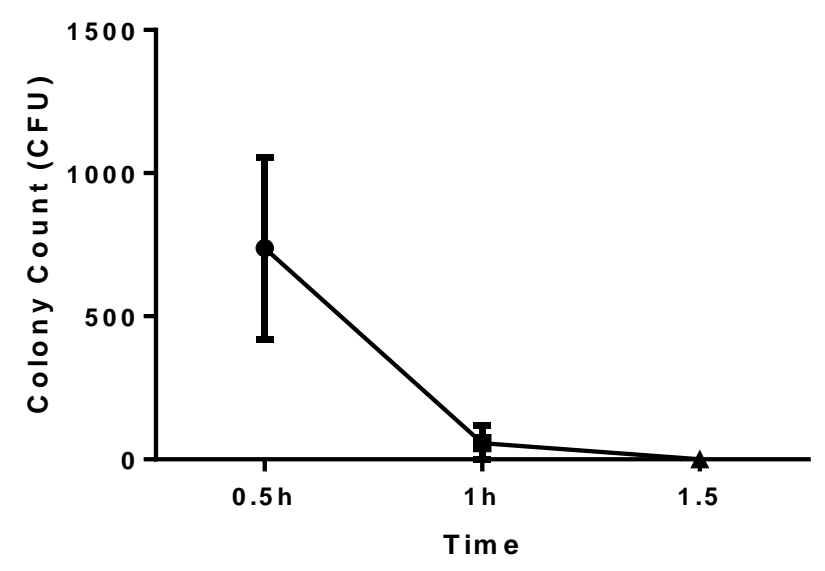

Figure 8.2. The effect of incubation with gentamicin on Campylobacter in PBS after 6 hours invasion with HT-29/Caco-2/8E11 cells

Table 8.1: $25 \%$ Subgroup of isolates used during Motility, Invasion and Adhesion assays, showing the presence of $f l a A / B, c d t A$ and $c i a B$ genes

\begin{tabular}{|l|lllll|}
\hline Isolate & ST & Source & flaA/B & cdtA & ciaB \\
\hline CampsClin11 & ST-45 & human & - & + & + \\
CampsClin45 & ST-45 & human & - & + & + \\
CampsClin583 & ST-45 & human & - & + & + \\
CampsClin883 & ST-21 & human & - & + & + \\
cow518 & ST-21 & cattle & + & + & + \\
chickc45 & ST-45 & chicken & + & + & + \\
chick19 & ST-21 & chicken & - & + & + \\
chick50 & ST-21 & chicken & - & + & + \\
chick53 & ST-21 & chicken & - & + & +
\end{tabular}




\begin{tabular}{l|lllll|} 
chick137 & ST-257 & chicken & - & + & + \\
chick2223 & ST-45 & chicken & + & + & + \\
cow3583 & ST-42 & cattle & - & + & + \\
cow618 & ST-61 & cattle & + & + & + \\
cow237 & ST-206 & cattle & - & + & + \\
cow270 & ST-403 & cattle & - & + & + \\
cow104 & ST-21 & cattle & + & + & + \\
cow3189 & & cattle & + & + & + \\
cow3207 & ST-45 & cattle & - & + & + \\
chick1079 & ST-573 & chicken & + & + & + \\
chickd45 & ST-45 & chicken & + & + & + \\
CampsClin21 & & human & + & + & + \\
OxClinb21 & ST-45 & human & - & + & + \\
OxClina45 & ST-45 & human & + & + & + \\
OxClinb45 & ST-21 & human & - & + & + \\
starling45 & ST-45 & starling & + & - & + \\
starling177 & ST-177 & starling & + & + & + \\
starling682 & ST-682 & starling & - & + & + \\
starling1020 & ST-682 & starling & + & + & + \\
goose1033 & ST- & goose & - & + & + \\
goose702 & 1034 & goose & + & - & - \\
goose137 & ST-45 & goose & + & + & + \\
goose696 & & & - & + & + \\
duck45 & ST-45 & duck & + & + & + \\
\hline
\end{tabular}

Table 8.2. Six Strains used to infect chickens experimentally

\begin{tabular}{|l|l|}
\hline Strain & Campylobacter species \\
\hline CJ13126 & C. jejuni \\
\hline CJ12662 & C. jejuni \\
\hline L4 & C. coli \\
\hline I4 & C. coli \\
\hline C8 & C. coli \\
\hline C15 & C. coli \\
\hline
\end{tabular}


Table 8.3. All genes related to human isolates identified with scoary

\begin{tabular}{|l|l|l|l|}
\hline Gene & Annotation & $\begin{array}{l}\text { Trait } \\
\text { positive } \\
\text { Isolates }\end{array}$ & $\begin{array}{l}\text { p } \\
\text { Number }\end{array}$ \\
\hline rppH & RNA pyrophosphohydrolase & 13 & 0.026217 \\
\hline hemH & Ferrochelatase & 13 & 0.026217 \\
\hline nlpA & Lipoprotein 28 & 12 & 0.031127 \\
\hline patA & Peptidoglycan O-acetyltransferase & 11 & 0.006454 \\
\hline$d s b L$ & Thiol:disulfide interchange protein DsbL & 11 & 0.024205 \\
\hline clpA & $\begin{array}{l}\text { ATP-dependent Clp protease ATP-binding subunit } \\
\text { ClpA }\end{array}$ & 11 & 0.024205 \\
\hline proP & Proline/betaine transporter & 10 & 0.010203 \\
\hline flgE & Flagellar hook protein FlgE & 8 & 0.005657 \\
\hline fabG & 3-oxoacyl-[acyl-carrier-protein] reductase FabG & 8 & 0.021386 \\
\hline aldA & Lactaldehyde dehydrogenase & 7 & 0.00667 \\
\hline hddC & $\begin{array}{l}\text { D-glycero-alpha-D-manno-heptose } \\
\text { guanylyltransferase }\end{array}$ & 7 & 0.035565 \\
\hline Ugd & UDP-glucose 6-dehydrogenase & 6 & 0.023647 \\
\hline$m s h A$ & D-inositol-3-phosphate glycosyltransferase & 6 & 0.032906 \\
\hline proP & Proline/betaine transporter & 5 & 0.008465 \\
\hline group_613 & putative inner membrane transporter YicL & 5 & 0.016809 \\
\hline hisC & Histidinol-phosphate aminotransferase & 4 & 0.006454 \\
\hline fliY & L-cystine-binding protein FliY & 3 & 0.021322 \\
\hline dcuD & $\begin{array}{l}\text { Putative cryptic C4-dicarboxylate transporter } \\
\text { DcuD }\end{array}$ & 2 & 0.026217 \\
\hline group_1649 & Colicin I receptor & 2 & 0.026217 \\
\hline dcuD & $\begin{array}{l}\text { Putative cryptic C4-dicarboxylate transporter } \\
\text { DcuD }\end{array}$ & 2 & 0.026217 \\
\hline pctC & Methyl-accepting chemotaxis protein McpB & 2 & 0.026217 \\
\hline rfbC & dTDP-4-dehydrorhamnose 3,5-epimerase & 0 & 0.019585 \\
\hline & & & \\
\hline
\end{tabular}

Table 8.4. All genes related to chicken isolated identified with scoary

\begin{tabular}{|l|l|l|l|}
\hline Gene & Annotation & Trait positive Isolates & $\begin{array}{l}\text { p } \\
\text { Number }\end{array}$ \\
\hline murG & $\begin{array}{l}\text { UDP-N-acetylglucosamine--N- } \\
\text { acetylmuramyl-(pentapeptide) } \\
\text { pyrophosphoryl-undecaprenol N- } \\
\text { acetylglucosamine transferase }\end{array}$ & 40 & 0.015864 \\
\hline$f l g R$ & $\begin{array}{l}\text { Sigma-54 associated transcriptional } \\
\text { activator }\end{array}$ & 39 & 0.039786 \\
\hline Cj1375 & Multidrug efflux transporter & 34 & 0.041552 \\
\hline$g r p E$ & Protein GrpE & 32 & 0.000992 \\
\hline$y m f D$ & Bacillibactin exporter & 31 & 0.015862 \\
\hline
\end{tabular}




\begin{tabular}{|l|l|l|l|}
\hline feoB & Ferrous iron transport protein B & 29 & 0.000442 \\
\hline appA & Oligopeptide-binding protein AppA & 27 & 0.000397 \\
\hline group_35 & $\begin{array}{l}\text { Putative polysaccharide biosynthesis } \\
\text { protein with aminopeptidase-like } \\
\text { domain protein }\end{array}$ & 25 & 0.010515 \\
\hline gloB & Hydroxyacylglutathione hydrolase & 23 & 0.000146 \\
\hline torC & Cytochrome c-type protein TorC & 21 & 0.006902 \\
\hline group_108 & $\begin{array}{l}\text { putative copper-importing P-type } \\
\text { ATPase A }\end{array}$ & 18 & 0.010152 \\
\hline group_543 & Cystathionine beta-lyase & 15 & 0.001326 \\
\hline group_255 & Ferrous iron transport protein B & 13 & 0.000397 \\
\hline group_1256 & Oligopeptide-binding protein AppA & 13 & 0.000397 \\
\hline group_941 & putative methyltransferase & 13 & 0.005506 \\
\hline group_2141 & putative tautomerase & 10 & 0.018056 \\
\hline group_647 & Bacillibactin exporter & 10 & 0.049141 \\
\hline group_1262 & Major outer membrane protein & 9 & 0.004334 \\
\hline group_2007 & Protein GrpE & 8 & 0.000992 \\
\hline group_40 & Sensor protein KdpD & 5 & 0.014972 \\
\hline rfbC & $\begin{array}{l}\text { dTDP-4-dehydrorhamnose } \\
\text { epimerase }\end{array}$ & 4 & 0.020262 \\
\hline group_8 & $\begin{array}{l}\text { Potassium-transporting ATPase ATP- } \\
\text { binding subunit }\end{array}$ & 4 & 0.035766 \\
\hline group_1998 & $\begin{array}{l}\text { Ubiquinone biosynthesis } \\
\text { methyltransferase }\end{array}$ & 4 & 0.035766 \\
\hline group_1615 & Flagellar hook protein FlgE & 4 & 0.035766 \\
\hline group_804 & putative glycosyltransferase EpsJ & 1 & 0.031687 \\
\hline group_831 & $\begin{array}{l}\text { Anaerobic C4-dicarboxylate transporter } \\
\text { DcuA }\end{array}$ & 1 & 0 \\
\hline hisC_2 & Histidinol-phosphate aminotransferase & 0 & \\
\hline
\end{tabular}

Table 8.5. All genes related to cattle isolates identified with scoary

\begin{tabular}{|l|l|l|l|}
\hline Gene & Annotation & $\begin{array}{l}\text { Trait positive } \\
\text { Isolates }\end{array}$ & p Number \\
\hline flgD & Basal-body rod modification protein FlgD & 25 & 0.030579 \\
\hline pyrC_l & Dihydroorotase & 25 & 0.030579 \\
\hline feoB & Ferrous iron transport protein B & 25 & 0.032227 \\
\hline panB & $\begin{array}{l}\text { 3-methyl-2-oxobutanoate } \\
\text { hydroxymethyltransferase }\end{array}$ & 24 & 0.000306 \\
\hline phnU & $\begin{array}{l}\text { Putative 2-aminoethylphosphonate } \\
\text { transport system permease protein PhnU }\end{array}$ & 24 & 0.002764 \\
\hline dgkA & Diacylglycerol kinase & 24 & 0.005679 \\
\hline panC & Pantothenate synthetase & 23 & 0.002187 \\
\hline panD & Aspartate 1-decarboxylase & 23 & 0.002187 \\
\hline
\end{tabular}




\begin{tabular}{|c|c|c|c|}
\hline$s b p$ & Sulfate-binding protein & 23 & 0.028673 \\
\hline gloB & Hydroxyacylglutathione hydrolase & 23 & 0.049344 \\
\hline yafP & putative N-acetyltransferase YafP & 22 & 0.002999 \\
\hline potA & $\begin{array}{l}\text { Sulfate/thiosulfate import ATP-binding } \\
\text { protein CysA }\end{array}$ & 22 & 0.006116 \\
\hline hxuB & $\begin{array}{l}\text { Heme/hemopexin transporter protein } \\
\text { HuxB }\end{array}$ & 22 & 0.006116 \\
\hline group_1747 & Beta-lactamase OXA-133 & 22 & 0.011435 \\
\hline hisJ & Putative hydrolase & 22 & 0.011944 \\
\hline$f a b D$ & $\begin{array}{l}\text { Malonyl CoA-acyl carrier protein } \\
\text { transacylase }\end{array}$ & 22 & 0.019578 \\
\hline torC & Cytochrome c-type protein TorC & 21 & 0.047026 \\
\hline torZ & Trimethylamine-N-oxide reductase 2 & 20 & 0.016152 \\
\hline group_941 & putative methyltransferase & 18 & 0.017605 \\
\hline ssal & Serotype-specific antigen 1 & 18 & 0.018562 \\
\hline porA & Major outer membrane protein & 17 & 0.004488 \\
\hline$r f b C$ & dTDP-4-dehydrorhamnose 3,5-epimerase & 10 & 0.021465 \\
\hline epsJ_2 & putative glycosyltransferase EpsJ & 9 & 0.044124 \\
\hline aldA & Lactaldehyde dehydrogenase & 7 & 0.009065 \\
\hline group_1011 & GDP-L-fucose synthase & 7 & 0.017522 \\
\hline group_794 & Inner membrane transport protein RhmT & 7 & 0.040523 \\
\hline group_831 & $\begin{array}{l}\text { Anaerobic C4-dicarboxylate transporter } \\
\text { DcuA }\end{array}$ & 6 & 0.025047 \\
\hline group_1670 & Flagellar hook protein FlgE & 5 & 0.016396 \\
\hline group_1636 & $\mathrm{N}$-acylneuraminate cytidylyltransferase & 5 & 0.016396 \\
\hline group_303 & GTPase Obg & 5 & 0.035108 \\
\hline group_169 & Bifunctional protein Aas & 4 & 0.019918 \\
\hline group_2390 & $\begin{array}{l}\text { D-glycero-alpha-D-manno-heptose } \\
\text { phosphate guanylyltransferase }\end{array}$ & 3 & 0.019578 \\
\hline group_2259 & putative metallo-hydrolase & 3 & 0.019578 \\
\hline $\lg \mathrm{g} D$ & Linear gramicidin synthase subunit D & 3 & 0.019578 \\
\hline$y d i O$ & $\begin{array}{l}\text { putative BsuMI modification methylase } \\
\text { subunit YdiO }\end{array}$ & 3 & 0.019578 \\
\hline group_108 & $\begin{array}{l}\text { putative copper-importing P-type ATPase } \\
\text { A }\end{array}$ & 1 & 0.000606 \\
\hline group_791 & Diacylglycerol kinase & 1 & 0.005679 \\
\hline group_1279 & Major outer membrane protein & 1 & 0.033251 \\
\hline group_255 & Ferrous iron transport protein B & 0 & 0.008874 \\
\hline group_461 & CRISPR-associated endonuclease Cas1 & 0 & 0.030579 \\
\hline cas9_2 & CRISPR-associated endonuclease Cas9 & 0 & 0.030579 \\
\hline
\end{tabular}

Table 8.6. All genes related to wild bird isolates identified with scoary

\begin{tabular}{|l|l|l|l|}
\hline Gene & Annotation & $\begin{array}{l}\text { Trait } \\
\text { positive } \\
\text { Isolates }\end{array}$ & p Number \\
\hline
\end{tabular}




\begin{tabular}{|c|c|c|c|}
\hline hrcA & $\begin{array}{l}\text { Heat-inducible transcription repressor } \\
\text { HrcA }\end{array}$ & 9 & 0.002728 \\
\hline hydA_3 & $\begin{array}{l}\text { Quinone-reactive } \quad \mathrm{Ni} / \mathrm{Fe}-\text { hydrogenase } \\
\text { small chain }\end{array}$ & 9 & 0.004608 \\
\hline group_924 & Sensor protein CpxA & 8 & 0.009051 \\
\hline group_464 & DNA ligase & 8 & 0.029978 \\
\hline$h d d A$ & $\begin{array}{l}\text { D-glycero-alpha-D-manno-heptose } \\
\text { phosphate kinase }\end{array}$ & 8 & 0.033468 \\
\hline$g m h A$ & Phosphoheptose isomerase & 8 & 0.036215 \\
\hline yajR & Inner membrane transport protein YajR & 7 & 0.025536 \\
\hline$v h b$ & Bacterial hemoglobin & 7 & 0.025536 \\
\hline fdoI & $\begin{array}{l}\text { Formate dehydrogenase, cytochrome } \\
\text { b556(fdo) subunit }\end{array}$ & 7 & 0.025536 \\
\hline $\operatorname{lpxL}$ & Lipid A biosynthesis lauroyltransferase & 7 & 0.04836 \\
\hline legF_l & $\begin{array}{l}\text { CMP-N,N'-diacetyllegionaminic acid } \\
\text { synthase }\end{array}$ & 7 & 0.04836 \\
\hline $\operatorname{car} B$ & $\begin{array}{l}\text { Carbamoyl-phosphate synthase large } \\
\text { chain }\end{array}$ & 7 & 0.04836 \\
\hline flaA & Flagellin A & 6 & 0.002712 \\
\hline fliD & Flagellar hook-associated protein 2 & 6 & 0.002712 \\
\hline$r f b C$ & dTDP-4-dehydrorhamnose 3,5-epimerase & 6 & 0.003282 \\
\hline group_791 & Diacylglycerol kinase & 6 & 0.004432 \\
\hline$y y c B$ & putative transporter $\mathrm{YycB}$ & 6 & 0.006428 \\
\hline tenI & Thiazole tautomerase & 6 & 0.006428 \\
\hline murG & $\begin{array}{l}\text { UDP-N-acetylglucosamine--N- } \\
\text { acetylmuramyl-(pentapeptide) } \\
\text { pyrophosphoryl-undecaprenol } \\
\text { acetylglucosamine transferase }\end{array}$ & 6 & 0.0202 \\
\hline exs $A$ & $\begin{array}{l}\text { Exoenzyme } \mathrm{S} \text { synthesis regulatory protein } \\
\text { ExsA }\end{array}$ & 6 & 0.030612 \\
\hline proA & Gamma-glutamyl phosphate reductase & 5 & 4.93E-05 \\
\hline ubiE & $\begin{array}{l}\text { Ubiquinone/menaquinone biosynthesis C- } \\
\text { methyltransferase UbiE }\end{array}$ & 5 & 0.000142 \\
\hline$m d a B$ & Modulator of drug activity B & 5 & 0.000235 \\
\hline group_1420 & putative histidine-binding protein & 5 & 0.000589 \\
\hline proB & Glutamate 5-kinase & 5 & 0.001494 \\
\hline group_1919 & Bacteriohemerythrin & 5 & 0.00395 \\
\hline$f \lg D$ & Basal-body rod modification protein FlgD & 5 & 0.011567 \\
\hline group_675 & Polysialic acid transport protein KpsM & 5 & 0.018378 \\
\hline group_2171 & Gamma-glutamyl phosphate reductase & 4 & 4.93E-05 \\
\hline$l d h$ & L-lactate dehydrogenase & 4 & $5.56 \mathrm{E}-05$ \\
\hline pyrC_l & Dihydroorotase & 4 & 0.001036 \\
\hline group_1661 & Basal-body rod modification protein FlgD & 4 & 0.007737 \\
\hline group_302 & Glutamate 5-kinase & 4 & 0.007737 \\
\hline ompR & Transcriptional regulatory protein OmpR & 4 & 0.007896 \\
\hline group_1600 & Bacteriohemerythrin & 4 & 0.010701 \\
\hline
\end{tabular}




\begin{tabular}{|c|c|c|c|}
\hline ubiG_1 & 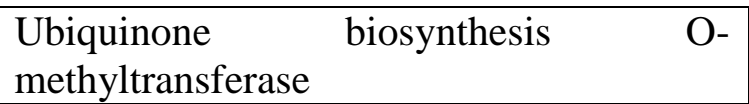 & 4 & 0.018378 \\
\hline kpsM & Polysialic acid transport protein KpsM & 4 & 0.02341 \\
\hline citN & Citrate transporter & 4 & 0.036242 \\
\hline group_2181 & Modulator of drug activity $\mathrm{B}$ & 3 & 0.000715 \\
\hline group_1674 & Dihydroorotase & 3 & 0.000715 \\
\hline group_1677 & Thiazole tautomerase & 3 & 0.002712 \\
\hline group_200 & Citrate transporter & 3 & 0.002712 \\
\hline$d g k A$ & Diacylglycerol kinase & 3 & 0.004432 \\
\hline group_1025 & putative transporter $\mathrm{YycB}$ & 3 & 0.006428 \\
\hline group_1303 & $\begin{array}{l}\text { UDP-N-acetylglucosamine--N- } \\
\text { acetylmuramyl-(pentapeptide) } \\
\text { pyrophosphoryl-undecaprenol } \\
\text { acetylglucosamine transferase }\end{array}$ & 3 & 0.006428 \\
\hline$s b p$ & Sulfate-binding protein & 3 & 0.007662 \\
\hline group_1671 & Bacteriohemerythrin & 3 & 0.012183 \\
\hline group_2170 & Phosphoethanolamine transferase EptA & 3 & 0.012183 \\
\hline group_310 & Trimethylamine-N-oxide reductase 2 & 3 & 0.030612 \\
\hline group_1662 & Beta-lactamase OXA-133 & 3 & 0.043478 \\
\hline phnU & $\begin{array}{l}\text { Putative } \\
\text { transport system permease protein PhnU }\end{array}$ & 2 & 0.000815 \\
\hline$z r a S \_2$ & Sensor protein ZraS & 2 & 0.001518 \\
\hline panB & $\begin{array}{l}\text { 3-methyl-2-oxobutanoate } \\
\text { hydroxymethyltransferase }\end{array}$ & 2 & 0.003453 \\
\hline panC & Pantothenate synthetase & 2 & 0.004429 \\
\hline panD & Aspartate 1-decarboxylase & 2 & 0.004429 \\
\hline hisJ & Putative hydrolase & 2 & 0.004429 \\
\hline group_1747 & Beta-lactamase OXA-133 & 2 & 0.00562 \\
\hline group_2175 & $\begin{array}{l}\text { UDP-4-amino-4,6-dideoxy-N-acetyl-beta- } \\
\text { L-altrosamine N-acetyltransferase }\end{array}$ & 2 & 0.008989 \\
\hline group_1684 & $\begin{array}{l}\text { D-glycero-alpha-D-manno-heptose } \\
\text { phosphate guanylyltransferase }\end{array}$ & 2 & 0.008989 \\
\hline group_2235 & Lipid A biosynthesis lauroyltransferase & 2 & 0.008989 \\
\hline group_2224 & Bacterial hemoglobin & 2 & 0.008989 \\
\hline group_2179 & GDP-L-fucose synthase & 2 & 0.008989 \\
\hline torZ_1 & Trimethylamine-N-oxide reductase 2 & 2 & 0.008989 \\
\hline yafP & putative $\mathrm{N}$-acetyltransferase YafP & 2 & 0.010829 \\
\hline group_1933 & $\begin{array}{l}\text { UDP-4-amino-4,6-dideoxy-N-acetyl-beta- } \\
\text { L-altrosamine N-acetyltransferase }\end{array}$ & 2 & 0.025536 \\
\hline group_2166 & putative adenylyl-sulfate kinase & 2 & 0.025536 \\
\hline group_1685 & dTDP-4-dehydrorhamnose 3,5-epimerase & 2 & 0.025536 \\
\hline group_326 & Biopolymer transport protein ExbB & 2 & 0.025536 \\
\hline $\operatorname{leg} F$ & $\begin{array}{l}\text { CMP-N,N'-diacetyllegionaminic } \\
\text { synthase }\end{array}$ & 2 & 0.025536 \\
\hline group_409 & Thiosulfate dehydrogenase & 2 & 0.025536 \\
\hline potA & Sulfate/thiosulfate import ATP-binding & 1 & 0.000688 \\
\hline
\end{tabular}




\begin{tabular}{|l|l|l|l|}
\hline & protein CysA & & \\
\hline cpxA & Sensor protein CpxA & 1 & 0.003831 \\
\hline ligA_2 & DNA ligase & 1 & 0.014107 \\
\hline lagD & $\begin{array}{l}\text { Lactococcin-G-processing and transport } \\
\text { ATP-binding protein LagD }\end{array}$ & 1 & 0.031524 \\
\hline
\end{tabular}

Table 8.7. All genes related to the caeca isolates identified with scoary

\begin{tabular}{|c|c|c|c|}
\hline Gene & Annotation & \begin{tabular}{|l} 
Trait \\
Positive \\
isolates
\end{tabular} & $\begin{array}{l}p \\
\text { Number }\end{array}$ \\
\hline$n r f H$ & Cytochrome c-type protein $\mathrm{NrfH}$ & 4 & 0.004012 \\
\hline pglJ_l & $\begin{array}{l}\text { N-acetylgalactosamine-N,N'- } \\
\text { diacetylbacillosaminyl-diphospho- } \\
\text { undecaprenol } \\
\text { acetylgalactosaminyltransferase }\end{array}$ & 4 & 0.016851 \\
\hline tils & tRNA(Ile)-lysidine synthase & 4 & 0.04234 \\
\hline putA_2 & Bifunctional protein PutA & 4 & 0.04234 \\
\hline$p b p G$ & Penicillin-binding protein 2D & 4 & 0.04234 \\
\hline$g l c C$ & Glc operon transcriptional activator & 4 & 0.04234 \\
\hline ifcA & Fumarate reductase flavoprotein subunit & 4 & 0.04234 \\
\hline feoA & Putative $\mathrm{Fe}(2)$ transport protein A & 4 & 0.04234 \\
\hline hhalM & Modification methylase HhaI & 4 & 0.04234 \\
\hline soj_l & Chromosome-partitioning ATPase Soj & 4 & 0.04234 \\
\hline yafQ & mRNA interferase YafQ & 4 & 0.04234 \\
\hline epsJ_1 & putative glycosyltransferase EpsJ & 4 & 0.04234 \\
\hline$k d s D$ & Arabinose 5-phosphate isomerase KdsD & 4 & 0.04234 \\
\hline proP_4 & Proline/betaine transporter & 4 & 0.04234 \\
\hline fhaB_3 & Filamentous hemagglutinin & 4 & 0.04234 \\
\hline$f c f 2$ & dTDP-fucopyranose mutase & 4 & 0.04234 \\
\hline intA_2 & Prophage integrase IntA & 4 & 0.04234 \\
\hline
\end{tabular}




\begin{tabular}{|c|c|c|c|}
\hline$m q n A \_l$ & Chorismate dehydratase & 4 & 0.04234 \\
\hline polC_2 & DNA polymerase III PolC-type & 4 & 0.04234 \\
\hline relG & Toxin RelG & 4 & 0.04234 \\
\hline $\operatorname{din} B$ & DNA polymerase IV & 4 & 0.04234 \\
\hline intA_1 & Prophage integrase Int $\mathrm{A}$ & 4 & 0.04234 \\
\hline$d p n A$ & Modification methylase DpnIIB & 4 & 0.04234 \\
\hline yxeP & putative hydrolase YxeP & 4 & 0.04234 \\
\hline$r f b C$ & dTDP-4-dehydrorhamnose 3,5-epimerase & 4 & 0.04234 \\
\hline kpsT & $\begin{array}{l}\text { Polysialic acid transport } \text { ATP-binding } \\
\text { protein KpsT }\end{array}$ & 4 & 0.04234 \\
\hline yneB & Resolvase YneB & 4 & 0.04234 \\
\hline kpsM & Polysialic acid transport protein KpsM & 4 & 0.04234 \\
\hline $\operatorname{lex} A$ & LexA repressor & 4 & 0.04234 \\
\hline bcs1 & $\begin{array}{l}\text { Bifunctional ribulose 5-phosphate } \\
\text { reductase/CDP-ribitol pyrophosphorylase } \\
\text { Bcs1 }\end{array}$ & 4 & 0.04234 \\
\hline$f c l$ & GDP-L-fucose synthase & 4 & 0.04234 \\
\hline$n r f A$ & Cytochrome c-552 & 3 & 0.018054 \\
\hline cymR & HTH-type transcriptional regulator CymR & 3 & 0.018054 \\
\hline gltB_6 & Glutamate synthase [NADPH] large chain & 3 & 0.018054 \\
\hline feoA & Putative $\mathrm{Fe}(2)$ transport protein $\mathrm{A}$ & 2 & 0.013814 \\
\hline group_10282 & tRNA-Val(tac) & 2 & 0.013814 \\
\hline$m s r P$ & $\begin{array}{l}\text { Protein-methionine-sulfoxide } \quad \text { reductase } \\
\text { catalytic subunit MsrP }\end{array}$ & 2 & 0.037489 \\
\hline$u v r B$ & UvrABC system protein B & 0 & 0.00869 \\
\hline$k d p A \_1$ & $\begin{array}{l}\text { Potassium-transporting ATPase potassium- } \\
\text { binding subunit }\end{array}$ & 0 & 0.017179 \\
\hline fic & monophosphate-protein & 0 & 0.037322 \\
\hline
\end{tabular}




\begin{tabular}{|l|l|l|l|}
\hline & transferase SoFic & & \\
\hline katA & Catalase & 0 & 0.037322 \\
\hline tmk_I & Thymidylate kinase & 0 & 0.040346 \\
\hline$b l c$ & Outer membrane lipoprotein Blc & 0 & 0.040346 \\
\hline$m s h A$ & D-inositol-3-phosphate glycosyltransferase & 0 & 0.040346 \\
\hline epsJ_5 & putative glycosyltransferase EpsJ & 0 & 0.040346 \\
\hline prkC & Serine/threonine-protein kinase PrkC & 0 & 0.040346 \\
\hline epsJ_2 & putative glycosyltransferase EpsJ & 0 & 0.040346 \\
\hline$u b i G_{-} 1$ & $\begin{array}{l}\text { Ubiquinone } \\
\text { methyltransferase }\end{array}$ & 0 & 0.040346 \\
\hline
\end{tabular}

Table 8.8. All genes related to the ileum isolates identified with scoary

\begin{tabular}{|l|l|l|l|}
\hline Gene & Annotation & $\begin{array}{l}\text { Trait } \\
\text { Positive } \\
\text { isolates }\end{array}$ & p Number \\
\hline metE & $\begin{array}{l}\text { 5-methyltetrahydropteroyltriglutamate-- } \\
\text { homocysteine methyltransferase }\end{array}$ & 10 & 0.031294 \\
\hline metQ__ & Membrane lipoprotein TpN32 & 10 & 0.031294 \\
\hline selB & Selenocysteine-specific elongation factor & 10 & 0.040742 \\
\hline folD & Bifunctional protein FolD protein & 10 & 0.040742 \\
\hline paeR7IM & Type IIS restriction enzyme Eco57I & 8 & 0.032662 \\
\hline infC & Translation initiation factor IF-3 & 2 & 0.037139 \\
\hline ubiG_l & Ubiquinone biosynthesis O-methyltransferase & 2 & 0.037139 \\
\hline uvrB & UvrABC system protein B & 2 & 0.037139 \\
\hline mdh & Malate dehydrogenase & 2 & 0.044913 \\
\hline group_4752 & N-carbamoyl-D-amino acid hydrolase & 2 & 0.044913 \\
\hline fieF & Ferrous-iron efflux pump FieF & 2 & 0.044913 \\
\hline
\end{tabular}




\begin{tabular}{|c|c|c|c|}
\hline$r s x B$ & Electron transport complex subunit RsxB & 2 & 0.044913 \\
\hline leuC & 3-isopropylmalate dehydratase large subunit & 2 & 0.044913 \\
\hline leuA & 2-isopropylmalate synthase & 2 & 0.044913 \\
\hline$c d t B$ & Cytolethal distending toxin subunit B & 2 & 0.044913 \\
\hline era & GTPase Era & 2 & 0.044913 \\
\hline $\operatorname{aguA}$ & Agmatine deiminase & 2 & 0.044913 \\
\hline$y b i T$ & $\begin{array}{l}\text { putative } \mathrm{ABC} \text { transporter } \\
\text { protein YbiT }\end{array}$ & 1 & 0.028571 \\
\hline aroC & Chorismate synthase & 1 & 0.028571 \\
\hline rpsL & 30S ribosomal protein $\mathrm{S} 12$ & 1 & 0.033572 \\
\hline$t m k \_2$ & Thymidylate kinase & 1 & 0.033572 \\
\hline fusA & Elongation factor $\mathrm{G}$ & 1 & 0.033572 \\
\hline pchF_2 & $\begin{array}{l}\text { 4-cresol dehydrogenase [hydroxylating] } \\
\text { flavoprotein subunit }\end{array}$ & 1 & 0.033572 \\
\hline uxaA_1 & Altronate dehydratase & 1 & 0.033572 \\
\hline$u x a A \_2$ & Altronate dehydratase & 1 & 0.033572 \\
\hline rhaM & L-rhamnose mutarotase & 1 & 0.033572 \\
\hline$b c r$ & Bicyclomycin resistance protein & 1 & 0.033572 \\
\hline dapA_2 & 4-hydroxy-tetrahydrodipicolinate synthase & 1 & 0.033572 \\
\hline panC & Pantothenate synthetase & 0 & 0.035204 \\
\hline
\end{tabular}

Table 8.9. All genes related to the liver isolates identified with scoary

\begin{tabular}{|l|l|l|l|}
\hline Gene & Annotation & $\begin{array}{l}\text { Trait Positive } \\
\text { isolates }\end{array}$ & p Number \\
\hline valS & Valine--tRNA ligase & 10 & 0.031294 \\
\hline mprA_2 & Response regulator MprA & 9 & 0.034523 \\
\hline infA & Translation initiation factor IF-1 & 8 & 0.00214 \\
\hline
\end{tabular}




\begin{tabular}{|c|c|c|c|}
\hline rhaM & L-rhamnose mutarotase & 8 & 0.007889 \\
\hline dapA_2 & 4-hydroxy-tetrahydrodipicolinate synthase & 8 & 0.007889 \\
\hline pchF_2 & $\begin{array}{l}\text { 4-cresol dehydrogenase [hydroxylating] } \\
\text { flavoprotein subunit }\end{array}$ & 8 & 0.007889 \\
\hline uxaA_2 & Altronate dehydratase & 8 & 0.007889 \\
\hline uxaA_l & Altronate dehydratase & 8 & 0.007889 \\
\hline gatA & $\begin{array}{l}\text { Glutamyl-tRNA(Gln) } \quad \text { amidotransferase } \\
\text { subunit A }\end{array}$ & 8 & 0.032662 \\
\hline epsN & $\begin{array}{l}\text { Putative pyridoxal phosphate-dependent } \\
\text { aminotransferase EpsN }\end{array}$ & 7 & 0.007706 \\
\hline ped & (S)-1-Phenylethanol dehydrogenase & 7 & 0.022056 \\
\hline fucP & L-fucose-proton symporter & 7 & 0.022056 \\
\hline aldA & Lactaldehyde dehydrogenase & 7 & 0.022056 \\
\hline meh_2 & Mesaconyl-C(4)-CoA hydratase & 7 & 0.022056 \\
\hline cys $W$ & $\begin{array}{l}\text { Sulfate transport system permease protein } \\
\text { CysW }\end{array}$ & 7 & 0.022056 \\
\hline$r h m T$ & Inner membrane transport protein $\mathrm{RhmT}$ & 7 & 0.022056 \\
\hline infA & Translation initiation factor IF-1 & 6 & 0.007706 \\
\hline cys $W$ & $\begin{array}{l}\text { Sulfate transport system permease protein } \\
\text { CysW }\end{array}$ & 6 & 0.007706 \\
\hline$t c d A$ & $\begin{array}{ll}\text { tRNA } & \text { threonylcarbamoyladenosine } \\
\text { dehydratase } & \end{array}$ & 6 & 0.007706 \\
\hline $\operatorname{pan} C$ & Pantothenate synthetase & 6 & 0.009811 \\
\hline epsN & $\begin{array}{l}\text { Putative pyridoxal phosphate-dependent } \\
\text { aminotransferase EpsN }\end{array}$ & 6 & 0.022056 \\
\hline panC & Pantothenate synthetase & 6 & 0.022056 \\
\hline panD & Aspartate 1-decarboxylase & 6 & 0.022056 \\
\hline surE & 5'-nucleotidase SurE & 6 & 0.022056 \\
\hline$r f b F$ & Glucose-1-phosphate cytidylyltransferase & 6 & 0.038591 \\
\hline
\end{tabular}




\begin{tabular}{|c|c|c|c|}
\hline$h x u B_{-} 1$ & Heme/hemopexin transporter protein $\mathrm{HuxB}$ & 6 & 0.038591 \\
\hline group_4331 & $\begin{array}{l}\text { PGL/p-HBAD } \\
\text { glycosyltransferase }\end{array}$ & 6 & 0.038591 \\
\hline gspA & General stress protein $\mathrm{A}$ & 6 & 0.038591 \\
\hline$r f b G$ & CDP-glucose 4,6-dehydratase & 6 & 0.038591 \\
\hline prk & Phosphoribulokinase & 6 & 0.038591 \\
\hline pglH_2 & $\begin{array}{l}\text { GalNAc-alpha-(1->4)-GalNAc-alpha-(1->3)- } \\
\text { diNAcBac-PP-undecaprenol alpha-1,4-N- } \\
\text { acetyl-D-galactosaminyltransferase }\end{array}$ & 6 & 0.038591 \\
\hline$c d i A$ & tRNA nuclease CdiA & 6 & 0.038591 \\
\hline$h c p C_{-} 1$ & Putative beta-lactamase HcpC & 6 & 0.038591 \\
\hline colC_l & GDP-L-colitose synthase & 6 & 0.038591 \\
\hline yigZ & IMPACT family member YigZ & 6 & 0.038591 \\
\hline colC_2 & GDP-L-colitose synthase & 6 & 0.038591 \\
\hline yigZ & IMPACT family member YigZ & 5 & 0.013114 \\
\hline glmU_2 & Bifunctional protein GlmU & 5 & 0.030207 \\
\hline cold__l & $\begin{array}{l}\text { GDP-4-keto-6-deoxy-D-mannose-3- } \\
\text { dehydratase / pyridoxamine-phosphate } \\
\text { transaminase }\end{array}$ & 5 & 0.030207 \\
\hline pgtP_2 & Phosphoglycerate transporter protein & 5 & 0.030207 \\
\hline$x e r H \_2$ & Tyrosine recombinase XerH & 5 & 0.032662 \\
\hline tcyP & L-cystine uptake protein TcyP & 4 & 0.008693 \\
\hline group_6824 & Non-canonical purine NTP pyrophosphatase & 4 & 0.008693 \\
\hline nuoN_2 & NADH-quinone oxidoreductase subunit $\mathrm{N}$ & 4 & 0.008693 \\
\hline$a d k \_2$ & Adenylate kinase & 4 & 0.008693 \\
\hline pta_2 & Phosphate acetyltransferase & 4 & 0.008693 \\
\hline pcrA_2 & ATP-dependent DNA helicase PcrA & 4 & 0.008693 \\
\hline uup_l & $\mathrm{ABC}$ transporter ATP-binding protein uup & 4 & 0.034523 \\
\hline
\end{tabular}




\begin{tabular}{|c|c|c|c|}
\hline mco_2 & Multicopper oxidase mco & 4 & 0.034523 \\
\hline alsT & Amino-acid carrier protein AlsT & 4 & 0.034523 \\
\hline hspR_2 & Putative heat shock protein HspR & 4 & 0.034523 \\
\hline mlaC & putative phospholipid-binding protein MlaC & 4 & 0.034523 \\
\hline$t t c A \_2$ & $\begin{array}{l}\text { tRNA 2-thiocytidine biosynthesis protein } \\
\text { TtcA }\end{array}$ & 4 & 0.034523 \\
\hline rsmI & 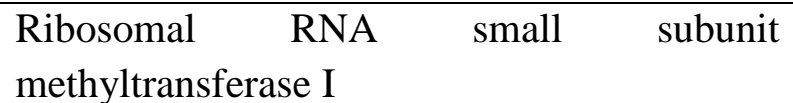 & 4 & 0.034523 \\
\hline $\operatorname{tarL}$ & $\begin{array}{l}\text { Teichoic acid poly(ribitol-phosphate) } \\
\text { polymerase }\end{array}$ & 4 & 0.034523 \\
\hline cbpA_2 & Curved DNA-binding protein & 4 & 0.034523 \\
\hline group_6190 & Farnesyl diphosphate synthase & 4 & 0.034523 \\
\hline aspS_2 & Aspartate--tRNA(Asp/Asn) ligase & 4 & 0.034523 \\
\hline tpfl & Antigen TpF1 & 4 & 0.034523 \\
\hline ppiD_2 & Peptidyl-prolyl cis-trans isomerase $\mathrm{D}$ & 4 & 0.034523 \\
\hline rpsA_3 & 30S ribosomal protein $\mathrm{S} 1$ & 4 & 0.034523 \\
\hline miaB_2 & $\begin{array}{l}\text { tRNA-2-methylthio-N(6)- } \\
\text { dimethylallyladenosine synthase }\end{array}$ & 4 & 0.034523 \\
\hline eno_2 & Enolase & 4 & 0.034523 \\
\hline tdeA_2 & Toxin and drug export protein $\mathrm{A}$ & 4 & 0.034523 \\
\hline argF_2 & Ornithine carbamoyltransferase & 4 & 0.034523 \\
\hline thy $X \_1$ & Flavin-dependent thymidylate synthase & 4 & 0.034523 \\
\hline nuoI_2 & NADH-quinone oxidoreductase subunit I & 4 & 0.034523 \\
\hline thyX_3 & Flavin-dependent thymidylate synthase & 4 & 0.034523 \\
\hline slyD_2 & $\begin{array}{l}\text { FKBP-type peptidyl-prolyl cis-trans } \\
\text { isomerase SlyD }\end{array}$ & 4 & 0.034523 \\
\hline recJ_2 & $\begin{array}{l}\text { Single-stranded-DNA-specific exonuclease } \\
\text { RecJ }\end{array}$ & 4 & 0.034523 \\
\hline
\end{tabular}




\begin{tabular}{|c|c|c|c|}
\hline fecE & $\begin{array}{l}\text { Iron(3 )-hydroxamate import ATP-binding } \\
\text { protein FhuC }\end{array}$ & 4 & 0.034523 \\
\hline rpsH_2 & 30S ribosomal protein $\mathrm{S} 8$ & 4 & 0.034523 \\
\hline group_8113 & tRNA-Phe(gaa) & 3 & 0.031294 \\
\hline$f a b Z \_2$ & $\begin{array}{l}\text { 3-hydroxyacyl-[acyl-carrier-protein] } \\
\text { dehydratase FabZ }\end{array}$ & 3 & 0.031294 \\
\hline$c f i B \_2$ & 2-oxoglutarate carboxylase small subunit & 3 & 0.031294 \\
\hline tupA_1 & Tungstate-binding protein TupA & 3 & 0.031294 \\
\hline kpsD_2 & Polysialic acid transport protein KpsD & 3 & 0.031294 \\
\hline cynT_2 & Carbonic anhydrase 1 & 3 & 0.031294 \\
\hline$p a b B \_1$ & $\begin{array}{l}\text { Aminodeoxychorismate synthase component } \\
1\end{array}$ & 3 & 0.031294 \\
\hline hisF_2 & $\begin{array}{l}\text { Imidazole glycerol phosphate synthase } \\
\text { subunit HisF }\end{array}$ & 3 & 0.031294 \\
\hline$d d l \_2$ & D-alanine--D-alanine ligase & 3 & 0.031294 \\
\hline$r p s G \_2$ & 30S ribosomal protein $\mathrm{S} 7$ & 3 & 0.031294 \\
\hline katA_2 & Catalase & 3 & 0.031294 \\
\hline$m k l$ & $\begin{array}{l}\text { putative ribonucleotide } \\
\text { binding protein } \mathrm{mkl}\end{array}$ & 3 & 0.031294 \\
\hline$d n a N_{-} 3$ & DNA polymerase III subunit beta & 3 & 0.031294 \\
\hline pheT_2 & Phenylalanine--tRNA ligase beta subunit & 3 & 0.031294 \\
\hline murG_2 & $\begin{array}{l}\text { UDP-N-acetylglucosamine--N- } \\
\text { acetylmuramyl-(pentapeptide) } \\
\text { pyrophosphoryl-undecaprenol } \\
\text { acetylglucosamine transferase }\end{array}$ & 3 & 0.031294 \\
\hline tolB_2 & Protein TolB & 3 & 0.031294 \\
\hline colD_4 & $\begin{array}{l}\text { GDP-4-keto-6-deoxy-D-mannose-3- } \\
\text { dehydratase / pyridoxamine-phosphate } \\
\text { transaminase }\end{array}$ & 3 & 0.031294 \\
\hline mlaA_2 & putative phospholipid-binding lipoprotein & 3 & 0.031294 \\
\hline
\end{tabular}




\begin{tabular}{|c|c|c|c|}
\hline & MlaA & & \\
\hline$y q g F \_2$ & Putative pre-16S rRNA nuclease & 3 & 0.031294 \\
\hline rpoZ_l & $\begin{array}{l}\text { DNA-directed RNA polymerase subunit } \\
\text { omega }\end{array}$ & 3 & 0.031294 \\
\hline$f b p \_3$ & Fructose-1,6-bisphosphatase class 1 & 3 & 0.031294 \\
\hline$n t h \_3$ & Endonuclease III & 3 & 0.031294 \\
\hline hisA_2 & $\begin{array}{l}\text { 1-(5-phosphoribosyl)-5-[(5- } \\
\text { phosphoribosylamino)methylideneamino] } \\
\text { imidazole-4-carboxamide isomerase }\end{array}$ & 3 & 0.031294 \\
\hline$r s h$ & $\begin{array}{l}\text { Bifunctional (p)ppGpp synthase/hydrolase } \\
\text { RelA }\end{array}$ & 3 & 0.031294 \\
\hline$s f r A \_2$ & NADPH-Fe(3 ) oxidoreductase subunit alpha & 3 & 0.031294 \\
\hline$m d o B$ & Phosphoglycerol transferase I & 3 & 0.031294 \\
\hline fliE & $\begin{array}{l}\text { Flagellar hook-basal body complex protein } \\
\text { FliE }\end{array}$ & 3 & 0.031294 \\
\hline$i l v B$ & Acetolactate synthase large subunit & 3 & 0.031294 \\
\hline serS_2 & Serine--tRNA ligase & 3 & 0.031294 \\
\hline vals_l & Valine--tRNA ligase & 3 & 0.031294 \\
\hline$i l v H \_2$ & $\begin{array}{lllll}\text { Acetolactate } & \text { synthase } & \text { isozyme } & 3 & \text { small } \\
\text { subunit }\end{array}$ & 3 & 0.031294 \\
\hline proC_2 & Pyrroline-5-carboxylate reductase & 3 & 0.031294 \\
\hline pgtP_l & Phosphoglycerate transporter protein & 3 & 0.031294 \\
\hline cirA_5 & Vitamin B12 transporter BtuB & 3 & 0.031294 \\
\hline pyrC_5 & Dihydroorotase & 3 & 0.031294 \\
\hline $\arg G_{-} l$ & Argininosuccinate synthase & 3 & 0.031294 \\
\hline group_6108 & Nitronate monooxygenase & 3 & 0.031294 \\
\hline$n r d A \_2$ & $\begin{array}{lll}\begin{array}{l}\text { Ribonucleoside-diphosphate } \\
\text { subunit alpha }\end{array} & \text { reductase } & 1 \\
\end{array}$ & 3 & 0.031294 \\
\hline ccoNl_2 & Cbb3-type cytochrome c oxidase subunit & 3 & 0.031294 \\
\hline
\end{tabular}




\begin{tabular}{|c|c|c|c|}
\hline & CcoN1 & & \\
\hline putP_2 & High-affinity proline transporter PutP & 3 & 0.031294 \\
\hline$r s s A \_2$ & $\begin{array}{l}\text { Swarming motility regulation sensor protein } \\
\text { RssA }\end{array}$ & 3 & 0.031294 \\
\hline$m l a E \_2$ & \begin{tabular}{|lll} 
putative phospholipid & $\mathrm{ABC}$ & transporter \\
permease protein MlaE & & \\
\end{tabular} & 3 & 0.031294 \\
\hline macB_5 & $\begin{array}{l}\text { Macrolide export ATP-binding/permease } \\
\text { protein MacB }\end{array}$ & 3 & 0.031294 \\
\hline$p g l E \_2$ & UDP-N-acetylbacillosamine transaminase & 3 & 0.031294 \\
\hline$a c c A \_2$ & $\begin{array}{l}\text { Acetyl-coenzyme A carboxylase carboxyl } \\
\text { transferase subunit alpha }\end{array}$ & 3 & 0.031294 \\
\hline$y y c B \_2$ & putative transporter $\mathrm{YycB}$ & 3 & 0.031294 \\
\hline hisH_2 & \begin{tabular}{|lll} 
Imidazole glycerol phosphate & synthase \\
subunit HisH & & \\
\end{tabular} & 3 & 0.031294 \\
\hline$p g l F \_2$ & $\begin{array}{l}\text { UDP-N-acetyl-alpha-D-glucosamine } \quad \text { C6 } \\
\text { dehydratase }\end{array}$ & 3 & 0.031294 \\
\hline pyrB_2 & Aspartate carbamoyltransferase & 3 & 0.031294 \\
\hline pglH_l & $\begin{array}{l}\text { GalNAc-alpha-(1->4)-GalNAc-alpha-(1->3)- } \\
\text { diNAcBac-PP-undecaprenol alpha-1,4-N- } \\
\text { acetyl-D-galactosaminyltransferase }\end{array}$ & 3 & 0.031294 \\
\hline groL_2 & $60 \mathrm{kDa}$ chaperonin & 3 & 0.031294 \\
\hline gluP & Rhomboid protease AarA & 3 & 0.031294 \\
\hline ruvA_2 & $\begin{array}{l}\text { Holliday junction ATP-dependent DNA } \\
\text { helicase RuvA }\end{array}$ & 3 & 0.031294 \\
\hline hprA & Putative 2-hydroxyacid dehydrogenase & 3 & 0.031294 \\
\hline sat_2 & Sulfate adenylyltransferase & 3 & 0.031294 \\
\hline mreB_l & Rod shape-determining protein MreB & 3 & 0.031294 \\
\hline rpoBC_5 & $\begin{array}{l}\text { Bifunctional DNA-directed RNA polymerase } \\
\text { subunit beta-beta' }\end{array}$ & 3 & 0.031294 \\
\hline gros_2 & $10 \mathrm{kDa}$ chaperonin & 3 & 0.031294 \\
\hline
\end{tabular}




\begin{tabular}{|c|c|c|c|}
\hline zupT_2 & Zinc transporter ZupT & 3 & 0.031294 \\
\hline rplI_1 & 50S ribosomal protein L9 & 3 & 0.031294 \\
\hline thiH_l & 2-iminoacetate synthase & 3 & 0.031294 \\
\hline fabD_2 & $\begin{array}{l}\text { Malonyl CoA-acyl carrier protein } \\
\text { transacylase }\end{array}$ & 3 & 0.031294 \\
\hline lapB_2 & Lipopolysaccharide assembly protein B & 3 & 0.031294 \\
\hline mprA_3 & Response regulator MprA & 3 & 0.031294 \\
\hline rpsB_l & 30S ribosomal protein $\mathrm{S} 2$ & 3 & 0.031294 \\
\hline korA_l & 2-oxoglutarate oxidoreductase subunit KorA & 3 & 0.031294 \\
\hline purB & Adenylosuccinate lyase & 3 & 0.031294 \\
\hline luxS_2 & S-ribosylhomocysteine lyase & 3 & 0.031294 \\
\hline$n r d E$ & $\begin{array}{l}\text { Ribonucleoside-diphosphate reductase } \\
\text { subunit alpha }\end{array}$ & 3 & 0.031294 \\
\hline $\operatorname{cor} A$ & Cytochrome c biogenesis protein CcsA & 3 & 0.031294 \\
\hline$h x c R$ & $\begin{array}{l}\text { putative type II secretion system protein } \\
\text { HxcR }\end{array}$ & 3 & 0.031294 \\
\hline galE & UDP-glucose 4-epimerase & 2 & 0.017283 \\
\hline epsM & Putative acetyltransferase EpsM & 2 & 0.017283 \\
\hline $\operatorname{alg} C$ & Phosphomannomutase/phosphoglucomutase & 2 & 0.037139 \\
\hline$r s f S$ & Ribosomal silencing factor RsfS & 2 & 0.037139 \\
\hline acpS & Holo-[acyl-carrier-protein] synthase & 2 & 0.037139 \\
\hline$k d p D \_2$ & Sensor protein $\mathrm{KdpD}$ & 2 & 0.044913 \\
\hline$h s d R \_2$ & Type-1 restriction enzyme $\mathrm{R}$ protein & 2 & 0.044913 \\
\hline group_8163 & putative type I restriction enzymeP M protein & 2 & 0.044913 \\
\hline artQ_2 & $\begin{array}{l}\text { Arginine transport system permease protein } \\
\text { ArtQ }\end{array}$ & 1 & 0.013019 \\
\hline$h x l R \_2$ & HTH-type transcriptional activator HxlR & 1 & 0.028571 \\
\hline$t m k \_2$ & Thymidylate kinase & 1 & 0.028571 \\
\hline
\end{tabular}




\begin{tabular}{|c|c|c|c|}
\hline$f a b G \_1$ & $\begin{array}{l}\text { 3-oxoacyl-[acyl-carrier-protein] reductase } \\
\text { FabG }\end{array}$ & 1 & 0.028571 \\
\hline$g s p A$ & General stress protein $\mathrm{A}$ & 1 & 0.028571 \\
\hline xerC & Tyrosine recombinase XerC & 1 & 0.028571 \\
\hline $\bmod A$ & Molybdate-binding periplasmic protein & 1 & 0.028571 \\
\hline$f d t A \_2$ & $\begin{array}{l}\text { TDP-4-oxo-6-deoxy-alpha-D-glucose-3,4- } \\
\text { oxoisomerase }\end{array}$ & 1 & 0.028571 \\
\hline$r f b B$ & dTDP-glucose 4,6-dehydratase & 1 & 0.028571 \\
\hline lexA_2 & LexA repressor & 1 & 0.028571 \\
\hline kgtP_2 & Alpha-ketoglutarate permease & 1 & 0.033572 \\
\hline$f d t A \_1$ & $\begin{array}{l}\text { TDP-4-oxo-6-deoxy-alpha-D-glucose-3,4- } \\
\text { oxoisomerase }\end{array}$ & 1 & 0.033572 \\
\hline fadD3 & $\begin{array}{l}\text { 3-[(3aS,4S,7aS)-7a-methyl-1,5-dioxo- } \\
\text { octahydro-1H-inden-4-yl]propanoyl:CoA } \\
\text { ligase }\end{array}$ & 1 & 0.033572 \\
\hline paeR7IM & Type IIS restriction enzyme Eco57I & 0 & 0.000937 \\
\hline hepA & $\begin{array}{l}\text { Heterocyst differentiation } \\
\text { protein HepA }\end{array}$ & 0 & 0.001375 \\
\hline
\end{tabular}

Table 8.10. List of cytokines measured in Human cytokine antibody array.

\begin{tabular}{|c|c|c|c|c|}
\hline & 1 & 3 & 5 & 7 \\
\hline $\mathbf{A}$ & Positive & IL- $1 \beta$ & IL-13 & RANTES \\
\hline B & Positive & IL-2 & IL-15 & TGF $\beta$ \\
\hline C & Negative & IL-3 & IL-16 & $\mathrm{TNF} \alpha$ \\
\hline D & Negative & IL-4 & IL-7A & $\mathrm{TNF} \beta$ \\
\hline $\mathbf{E}$ & $\begin{array}{l}\text { Eotaxin-1 } \\
\text { (CCL11) }\end{array}$ & IL-6 & IP-10 & TNF RI \\
\hline $\mathbf{F}$ & $\begin{array}{l}\text { Eotaxin-2 } \\
\text { (CCL24) }\end{array}$ & IL-6R & MCP-1 & TNF RII \\
\hline $\mathbf{G}$ & GCSF & IL-7 & MCP-2 & PDGF-BB \\
\hline $\mathbf{H}$ & GM-CSf & IL-8 & M-CSF & TIMP-2 \\
\hline I & ICAM-1 & IL-10 & MIG & Blank \\
\hline $\mathbf{J}$ & $\mathrm{IFN} \gamma$ & IL-11 & MIP-1 $1 \alpha$ & Blank \\
\hline $\mathbf{K}$ & I-309 & IL-12 p40 & MIP-1 $\beta$ & Negative \\
\hline $\mathbf{L}$ & IL- $1 \alpha$ & IL-12 p70 & MIP-1 $\delta$ & Positive \\
\hline
\end{tabular}

